JLAB-PHY-16-2199

SLAC-PUB-16448

\title{
The QCD Running Coupling
}

\author{
Alexandre Deur \\ Thomas Jefferson National Accelerator Facility, Newport News, VA 23606, USA
}

Stanley J. Brodsky

SLAC National Accelerator Laboratory, Stanford University, Stanford, CA 94309, USA

Guy F. de Téramond

Universidad de Costa Rica, San José, Costa Rica

April 27, 2016

\author{
deurpam@jlab.org, \\ sjbth@slac.stanford.edu, gdt@asterix.crnet.cr,
}

(Invited review article to be published in Progress in Particle and Nuclear Physics) 


\begin{abstract}
We review the present theoretical and empirical knowledge for $\alpha_{s}$, the fundamental coupling underlying the interactions of quarks and gluons in Quantum Chromodynamics (QCD). The dependence of $\alpha_{s}\left(Q^{2}\right)$ on momentum transfer $Q$ encodes the underlying dynamics of hadron physics -from color confinement in the infrared domain to asymptotic freedom at short distances. We review constraints on $\alpha_{s}\left(Q^{2}\right)$ at high $Q^{2}$, as predicted by perturbative QCD, and its analytic behavior at small $Q^{2}$, based on models of nonperturbative dynamics. In the introductory part of this review, we explain the phenomenological meaning of the coupling, the reason for its running, and the challenges facing a complete understanding of its analytic behavior in the infrared domain. In the second, more technical, part of the review, we discuss the behavior of $\alpha_{s}\left(Q^{2}\right)$ in the high momentum transfer domain of QCD. We review how $\alpha_{s}$ is defined, including its renormalization scheme dependence, the definition of its renormalization scale, the utility of effective charges, as well as "Commensurate Scale Relations" which connect the various definitions of the QCD coupling without renormalization-scale ambiguity. We also report recent significant measurements and advanced theoretical analyses which have led to precise QCD predictions at high energy. As an example of an important optimization procedure, we discuss the "Principle of Maximum Conformality", which enhances QCD's predictive power by removing the dependence of the predictions for physical observables on the choice of theoretical conventions such as the renormalization scheme. In the last part of the review, we discuss the challenge of understanding the analytic behavior $\alpha_{s}\left(Q^{2}\right)$ in the low momentum transfer domain. We survey various theoretical models for the nonperturbative strongly coupled regime, such as the light-front holographic approach to QCD. This new framework predicts the form of the quark-confinement potential underlying hadron spectroscopy and dynamics, and it gives a remarkable connection between the perturbative QCD scale $\Lambda$ and hadron masses. One can also identify a specific scale $Q_{0}$ which demarcates the division between perturbative and nonperturbative QCD. We also review other important methods for computing the QCD coupling, including lattice QCD, the Schwinger-Dyson equations and the GribovZwanziger analysis. After describing these approaches and enumerating their conflicting predictions, we discuss the origin of these discrepancies and how to remedy them. Our aim is not only to review the advances in this difficult area, but also to suggest what could be an optimal definition of $\alpha_{s}\left(Q^{2}\right)$ in order to bring better unity to the subject.
\end{abstract}




\section{Contents}

1 Preamble 4

2 Phenomenological overview: QCD and the behavior of $\alpha_{s} \quad 7$

3 The strong coupling $\alpha_{s}$ in the perturbative domain 14

3.1 Purpose of the running coupling . . . . . . . . . . . . 15

3.2 The evolution of $\alpha_{s}$ in perturbative QCD $\ldots \ldots \ldots \ldots$

3.3 Quark thresholds . . . . . . . . . . . . . . . . . . . 21

3.4 Computation of the pQCD effective coupling . . . . . . . . 22

3.5 Renormalization group . . . . . . . . . . . . . . . . 24

3.6 The Landau pole and the QCD parameter $\Lambda \ldots \ldots \ldots$

3.7 Improvement of the perturbative series . . . . . . . . . . . 26

3.7.1 Effective charges and commensurate scale relations . . . . . 27

3.7.2 The Brodsky, Lepage and Mackenzie procedure and its extensions 30

3.7.3 Principle of maximum conformality . . . . . . . . . . . . 32

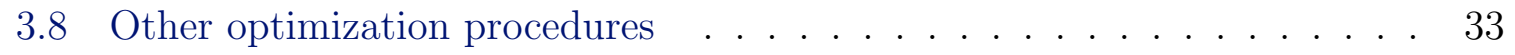

3.9 Determination of the strong coupling $\alpha_{s}\left(M_{Z}^{2}\right)$ or the QCD scale $\Lambda \ldots . \quad 35$

3.9.1 Deep Inelastic Scattering _ . . . . . . . . . . . . . 37

3.9.2 Observables from $e^{+} e^{-}$collisions . . . . . . . . . . . . . . 39

3.9.3 Observables from $p p$ collisions . . . . . . . . . . . . . . 42

3.9 .4 Lattice QCD . . . . . . . . . . . . . . . . . . . . 42

3.9 .5 Heavy quarkonia . . . . . . . . . . . . . . . . . . 49

3.9 .6 Holographic QCD . . . . . . . . . . . . . . . . . . . . 49

3.9 .7 Pion decay constant . . . . . . . . . . . . . 51

3.9 .8 Grand unification . . . . . . . . . . . . . . . . 51

3.9 .9 Comparison and discussion . . . . . . . . . . . . . . . . 52 
4 The strong coupling in the nonperturbative domain $\quad 54$

4.1 Effective charges . . . . . . . . . . . . . . . . . . . . 59

4.1.1 Measurement of the effective charge from the Bjorken sum rule. . 61

4.1.2 Measurement of the effective charge defined from $e^{+} e^{-}$annihilation. 64

4.1 .3 Sudakov effective charges . . . . . . . . . . . . . . 64

4.2 AdS/CFT and Holographic QCD . . . . . . . . . . . . 66

4.3 The effective potential approach . . . . . . . . . . . . . 70

4.3.1 The static Q- $\overline{\mathrm{Q}}$ potential . . . . . . . . . . . . . 70

4.3.2 Constraints on the running coupling from the hadron spectrum . 72

4.4 The Schwinger-Dyson formalism . . . . . . . . . . . . . . . 74

4.4.1 The QCD coupling defined from Schwinger-Dyson equations . . . 76

4.4 .2 Gauge choices . . . . . . . . . . . . . . 77

4.4.3 Classes of solutions in the IR domain . . . . . . . . . . . 78

4.4.4 The massive gluon propagator f . . . . . . . . . . 81

4.4.5 The coupling defined from the ghost-gluon vertex . . . . . . . 86

4.4.6 Couplings defined from the 3 -gluon and 4-gluon vertices . . . . 88

4.4 .7 Including quarks $\ldots \ldots \ldots \ldots \ldots$

4.4 .8 Gauge dependence . . . . . . . . . . . . . . . . . . . . . 91

4.5 Lattice gauge theory . . . . . . . . . . . . . . . . . . . . . . . 91

4.5.1 Lattice results for $\alpha_{s}$ in the IR . . . . . . . . . . . . . . . . 94

4.6 Functional renormalization group equations . . . . . . . . . . . 96

4.7 The Gribov-Zwanziger approach . . . . . . . . . . . . . . . . . . . . . 98

4.8 Stochastic quantization . . . . . . . . . . . . . . . . . 100

4.9 Analytic and dispersive approaches . . . . . . . . . . . . . . 102

4.9 .1 Analytic approach . . . . . . . . . . . . . . . . 102

4.9 .2 Dispersive approach . . . . . . . . . . . . . 106

4.10 Background perturbation theory . . . . . . . . . . . . 108

4.11 Optimized perturbation theory . . . . . . . . . . . . . . . 109

4.12 Quark-hadron duality . . . . . . . . . . . . . . . . . . 109

4.13 The IR mapping of $\lambda \phi^{4}$ to Yang-Mills theories . . . . . . . . . . . 110

4.14 The Bogoliubov compensation principle . . . . . . . . . . . . . 110

4.15 Curci-Fermi model . . . . . . . . . . . . . . . . . . . . . 110

5 Comparison and discussion $\quad 111$

5.1 Validity of the comparison . . . . . . . . . . . . . . . 111 
5.2 Influence of the renormalization scheme . . . . . . . . . . . . 116

5.3 The contributions of nonperturbative terms to $\alpha_{s}\left(Q^{2}\right) \ldots \ldots$. . . . . 118

5.4 Listing of the multiple IR-behavior found in the literature . . . . . . . . 122

6 Conclusions

A Lexicon 


\section{Chapter 1}

\section{Preamble}

We review the status of the coupling $\alpha_{s}\left(Q^{2}\right)$, the function which sets the strength of the interactions involving quarks and gluons in quantum chromodynamics (QCD), the fundamental gauge theory of the strong interactions, as a function of the momentum transfer $Q$. It is necessary to understand the behavior and magnitude of the QCD coupling over the complete $Q^{2}$ range in order to describe hadronic interactions at both long and short distances. At high $Q^{2}$ (short distances) precise knowledge of $\alpha_{s}\left(Q^{2}\right)$ is needed to match the growing accuracy of hadron scattering experiments as well as to test high-energy models unifying strong and electroweak forces. For example, uncertainties in the value of $\alpha_{s}\left(Q^{2}\right)$ contribute to the total theoretical uncertainty in the physics probed at the Large Hadron Collider, such as Higgs production via gluon fusion. It is also necessary to know the behavior of $\alpha_{s}$ at low $Q^{2}$ (long distances), such as the scale of the proton mass, in order to understand hadronic structure, quark confinement and hadronization processes. For example, processes involving the production of heavy quarks near threshold require the knowledge of the QCD coupling at low momentum scales. Even reactions at high energies may involve the integration of the behavior of the strong coupling over a large domain of momentum scales. Understanding the behavior of $\alpha_{s}$ at low $Q^{2}$ also allows us to reach a long-sought goal of hadron physics: to establish an explicit relation between the long distance domain characterized by quark confinement, and the short-distance regime where perturbative calculations are feasible. For such endeavor, a major challenge is to relate the parameter $\Lambda$, which controls the predictions of perturbative QCD (pQCD) at short distances, to the mass scale of hadrons. We will show later in this review how new theoretical insights into the behavior of QCD at large distances lead to an analytical relation between hadronic masses and $\Lambda$. This problem also involves one of the fundamental questions of QCD: How the mass scale for hadrons, 
such as the proton and $\rho$ meson, can emerge in the limit of zero-quark masses since no explicit mass scale appears in the QCD Lagrangian.

The origin and phenomenology of the behavior of $\alpha_{s}\left(Q^{2}\right)$ at small distances, where asymptotic freedom appears, is well understood and explained in many textbooks on Quantum Field Theory and Particle Physics. Numerous reviews exist; see e.g. Refs. $[1,2,3]$, some of which cover the long-distance behavior as well, e.g. Ref. [2]. However, standard explanations often create an apparent conundrum, as will be addressed in this review. Other questions remain even in this well understood regime: a significant issue is how to identify the scale $Q$ which controls a given hadronic process, especially if it depends on many physical scales. A fundamental requirement -called "renormalization group invariance" - is that physical observables cannot depend on the choice of the renormalization scale. We will briefly describe a new method, "the Principle of Maximum Conformality" (PMC), which can be used to set the renormalization scale unambiguously order-by-order in pQCD, while satisfying renormalization group invariance. The PMC method reduces, in the Abelian limit $N_{C} \rightarrow 0$, to the standard Gell-Mann-Low method which is used to obtain precise predictions for QED. Although $\alpha_{s}$ is well understood at small distances, it is much less so at long distances where it is related to color confinement. As Grunberg has emphasized [4], the QCD coupling can be defined from any physical observable which is perturbatively calculable. This is in analogy with QED, where the conventional Gell-Mann-Low coupling $\alpha\left(Q^{2}\right)$ is defined from the potential underlying the scattering amplitude of two heavy charged particles. Couplings defined following Grunberg's prescription are called "effective charges". They can be related to each other analytically via "Commensurate Scale Relations" and can be used at low $Q^{2}$. We will also review other possible definitions. An important example of a well measured effective charge, is the coupling $\alpha_{g_{1}}\left(Q^{2}\right)$, which is defined from the Bjorken sum rule for polarized deep inelastic lepton-proton scattering.

We will start this review with a phenomenological description of the behavior of $\alpha_{s}\left(Q^{2}\right)$. We will then continue with a theoretical discussion of its behavior at small distances based on the renormalization group equations and various approaches which can improve the standard formalism. We conclude this first section with the experimental and numerical measurements of $\alpha_{s}$ at short distances. We then discuss $\alpha_{s}$ at long distances, and survey several nonperturbative approaches which have been used to predict its behavior. We conclude this section with a comparison and discussion of the various approaches.

The level of this review is aimed for the main part at non-specialists and advanced 
graduate students. In that spirit, a lexicon specific to the study of the QCD running coupling and related topics is given in the Appendix. Words from this list are italicized.

We have endeavored to be as extensive as possible in this review, but since the study of $\alpha_{s}$ has been a very broad and active subject of research, the thousands of articles relevant to $\alpha_{s}$ quickly quench any ambition of an exhaustive review. Some topics closely related to $\alpha_{s}$, such as studies of the gluon propagator, renormalon phenomena, and higher-twist effects will only be mentioned; each of these topic deserves a review on its own. Likewise, $\alpha_{s}$ at finite temperature or in the time-like domain will not be discussed. Our choice of topics is subjective, and we apologize in advance to the specialists whose work has not been covered; there is no implication regarding their importance and validity. As usual, natural units $\hbar=c=1$ are used. 


\section{Chapter 2}

\section{Phenomenological overview: QCD and the behavior of $\alpha_{s}$}

In this chapter, we will present an introduction to the behavior of $\alpha_{s}\left(Q^{2}\right)$ from the soft to the hard domain A reader already familiar with this topic can skip this chapter; its purpose is to initiate the curious reader into the subject.

In Abelian quantum electrodynamics (QED), the running coupling

$$
\alpha=\frac{\alpha(0)}{1-\Pi\left(Q^{2}\right)}
$$

modifies the bare coupling $\bar{\alpha}=\frac{e^{2}}{4 \pi}$ appearing in the QED Lagrangian by incorporating the renormalization of the photon propagator with virtuality $q^{2}=-Q^{2}$. It sums all vacuum polarization insertions $\Pi\left(Q^{2}\right)$ starting with the familiar Serber-Uehling contribution from lepton pairs. The logarithmic ultraviolet (UV) divergence arising from vacuum polarization loop integration is conventionally regulated by the Pauli-Villars (massive-photon subtraction) method. The UV divergence is eliminated when one normalizes the coupling by experiment at a specific momentum $Q_{0}$. The UV divergences from the vertex renormalization factor $Z_{1}$ and the fermion propagator renormalization factor $Z_{2}$ cancel by the QED Ward-Takahashi identity. Consequently, only $Z_{3}$ from the virtual photon propagator requires renormalization.

In a general renormalizable quantum field theory (QFT), the coupling constant controlling the strength of the interactions described by the Lagrangian acquires a scale dependence after regularization of UV divergent integrals and the renormalization procedure. Typically, one uses dimensional regularization to define the divergent integrals 
since the procedure is gauge invariant. The UV cut-off dependence of the coupling is then eliminated by allowing the couplings and masses which appear in the Lagrangian to acquire a scale dependence and by normalizing them to a known (measured) value at a given scale. This "renormalization" procedure defines the running couplings (and running masses); in effect, they are effective couplings.

The origin of the ultraviolet divergences is often interpreted as a manifestation that a QFT is a low energy effective theory of more fundamental yet unknown theory. The UV cut-offs shield the very short distance domain where the QFT perhaps ceases to be valid. After normalizing the coupling to a measured value, the effective coupling is not sensitive to the ultraviolet (UV) cut-off and unknown phenomena arising beyond this scale. Thus the scale dependence of the coupling can be well understood formally and phenomenologically.

We illustrate this behavior for the coupling that arises in the static case of heavy sources and which provides a simple physical picture. Historically, and in the case of linear theories with massless force carriers, a force coupling constant is a universal coefficient that links the force to the "charges" of two bodies (e.g., the electric charge for electricity or the mass for gravity) divided by the distance dependence $1 / r^{2}$. The $1 / r^{2}$ dependence was classically interpreted as the weakening of the force flux as it spreads uniformly through space. In QFT this is interpreted as the manifestation in coordinate space of the propagator of the force mediator; the propagator is proportional to $1 / q^{2}$ in momentum space in the first Born approximation (i.e., one boson exchange, with $q$ the 4-momentum of the exchanged boson). For weak enough forces, the first Born approximation dominates higher order contributions and the $1 / q^{2}$ propagator in momentum yields the familiar $1 / r^{2}$ factor in coordinate space. However, higher orders do contribute and deviations from the $1 / r^{2}$ law thus occur. This extra $r$-dependence is folded in the coupling which then acquires a scale dependence.

In $\mathrm{QED}$, the contributions to the $Q^{2}$-dependence of the coupling only come from the vacuum polarization graphs. In QCD, the vacuum polarization (Fig. 2.1), the quark self-energy (Fig. 2.2a), the vertex corrections, and the gluon loop corrections to the elementary three-gluon and four-gluon couplings, (Fig. 2.2b) all contribute. (The separation of the amplitude in different graphs depends on convention. Hence, the statement of which graphs contribute has some arbitrariness. For example, the vertex corrections, Fig. 2.2b, do not contribute in the Landau gauge, $\partial_{\mu} A^{\mu}=0$ ). Tadpole graphs (Fig. 2.2c) do not contribute. Virtual emissions, where the gluon is reabsorbed by the rest of the hadron do not contribute to the coupling renormalization if they are 
UV finite. Since higher order quantum effects are responsible for the deviation from the $1 / r^{2}$ law, we expect the scale dependence to be significant only at a microscopic scale. Superficially, this discussion seems inapplicable to QCD, since it is a confining theory irrelevant to macroscopic scales. However, the non-Abelian nature of QCD induces a weakly-coupled QCD regime at small distances in which the first Born approximation is relevant and where the above discussion applies.

Having linked the deviation of the force from the $1 / r^{2}$ law to vacuum polarization and other processes, we can go further and explain the direction toward which a force deviates from $1 / r^{2}$. In QED pairs of particle-antiparticle are created around a test charge $q_{c}$. The particles of charge opposite to $q_{c}$ will tend to be closer to the test charge, see Fig. 2.3a. This is analogous to electric charge screening in a dielectric medium, see Fig. 2.3b. In both cases, by Gauss' law, the total charge inside a sphere of radius $r$ is smaller. The larger $r$, the more the total charge will tend to the test charge value $q_{c}$. From this, one sees that the magnitude of the running coupling in QED will decrease at larger distances, tending to its macroscopic value $\alpha(0) \simeq 1 / 137$, and get stronger at small distances. For QCD the situation is different because gluons carry color charges. The gluon linking the test color charge to a particle-antiparticle pair will have carried away the initial color of the test charge, see Fig. 2.1. Thus, the gluons tends to spatially dilute the initial test charge: the initial red quark in Fig. 2.1a spends most of its time as a green or blue quark, thus invisible to any high resolution gluons carrying an anti-red color that would normally interact with it. As a result, the opposite effect to QED happens (anti-screening). Since this effect dominates over the QED-like screening effect from quark-antiquark loops, the QCD running coupling tends to decrease with small distances. This gives physical insight into the phenomenon of "asymptotic freedom" ("ultraviolet" UV regime) at high momentum transfer and to a strong coupling regime in the low momentum transfer regime ("infrared" IR regime).

Formally, the running of a coupling originates from the renormalization procedure. However, predictions for observables must be independent of the choice of renormalization scheme (RS). This invariance under the choice of procedures forms a symmetry group, which allows techniques from group theory to be used to establish the behavior of $\alpha_{s}$. However, $\alpha_{s}$ itself needs not to be an observable, apart from particular cases such as the measure of the static QCD potential. Usually $\alpha_{s}$ is an expansion parameter in the perturbative series describing an observable $R$ : Typically

$$
R=\sum_{n} r_{n}\left(\frac{\alpha_{s}}{\pi}\right)^{n} .
$$


Although $R$ is RS-independent, the series coefficients, and thus $\alpha_{s}$, will depend on the scheme choice. However, because of the asymptotic freedom, $R \rightarrow r_{0}$ when the distance scale at which the observable is measured tends to zero. Thus the first coefficient of the series must be RS-independent. Formally at very high scales, the RS-dependence becomes minimal. Conversely, one anticipates that the dependence becomes increasingly important as larger distances are probed.

As we shall discuss in Section 3.7.3, one can use an analytic procedure, the Principle of Maximum Conformality, to make predictions for physical observables which are independent of the choice of RS.

The fact that $\alpha_{s}$ is not in general an observable and that it becomes increasingly dependent on the RS as the distance scale increases are important facts that must be kept in mind, especially since these effects in the prototypical quantum field theory -QEDare small or irrelevant. In general, the QCD effective coupling is considered to be only an intermediate quantity without precise physical meaning; if the coupling varies, e.g. by a factor of three depending on the RS choice, how can it be associated to the physical strength of the strong force? The advantages of viewing $\alpha_{s}$ as an intermediate nonphysical quantity are that unintuitive features such as RS-dependence or divergences (Landau pole) can be ignored, as long as observables are studied at small enough distances. However, this causes a conundrum: if $\alpha_{s}$ is not an observable and depends on arbitrary choices, how relevant is the phenomenological description given previously? This will be explained in Section 3.7.1 where we will see how $\alpha_{s}$ can be restored to the status of an observable. Advantages of such approach are that $\alpha_{s}$ re-acquires a physical and intuitive meaning, and that $\alpha_{s}$ can be defined at long distances.

So far, the discussion applies to the short-distance, weak-coupling, regime of QCD, where pQCD is applicable. What happens at large distances? Perturbative QCD suggests that at the confinement scale $\Lambda, \alpha_{s}$ becomes infinite, the so-called Landau pole. In the past, this behavior was viewed responsible for inducing the so-called "IR slavery" which keeps the quarks confined. In other words, quark confinement has been seen as a direct consequence of the Landau pole. However, the modern view is that, while UV freedom thrives, IR slavery should be abolished: the pQCD prediction of the Landau pole is irrelevant to the physical behavior of $\alpha_{s}$ since pQCD breaks down when $\alpha_{s} / \pi$ becomes close to unity. It may be useful to remember here that the coupling may not need to become infinite for a theory to be confining. For example the coupling is finite in $\mathrm{QCD}(1+1)$ which is a confining theory.

The subject of the IR-behavior of $\alpha_{s}$ is interesting and difficult. It will be discussed 
in the second part of this review. Instead of displaying a Landau pole, we expect (with the insight of numerous studies) that the strong increase of $\alpha_{s}$ should stop at some infrared scale, since its running stems from the colored particle/antiparticle loop creations, but the wavelengths of those particles cannot exceed the physical hadron size [6]. Hence, loops become suppressed in the infrared (IR) limit and $\alpha_{s}$ should become scale independent. This IR behavior is called the "freezing of $\alpha_{s}$ ". The following simple example illustrates the naive misconception that the divergence of the perturbative coupling at the Landau pole is responsible for quark confinement: the static Q- $\overline{\mathrm{Q}}$ potential displays a linear dependence up to a distance of $1.3 \mathrm{fm}$ in the physical case, or larger distances for pure field theory calculations without light quarks, see Section 4.3. However, as will be discussed in Section 4, the same theoretical calculations indicate that $\alpha_{s}$ becomes independent of the distance above $0.4 \mathrm{fm}$. It thus cannot be directly responsible for the continuing linear rise of the potential which keeps quarks and gluons confined. Indeed, rather than vacuum polarization loops increasing the coupling characterizing a one-gluon exchange, multi-gluon exchange between quarks, such as the H-graphs (see Fig. 2.2d) which are infrared divergent $[7,8]$, seems to be relevant to confinement.

To summarize this phenomenological description, $\alpha_{s}$ behaves as following: the coupling exhibits weak (inverse logarithmic) scale dependence at distances much smaller than $\Lambda^{-1}$, typically smaller than $10^{-16} \mathrm{~m}$. This is due to color charge spreading (asymptotic freedom properties). This weak behavior grows into a strong scale dependence at distances larger than a tenth of a Fermi. Finally, scale independence is restored at larger distances due to the confinement of quarks and gluons. The fact that there is a region where the strong coupling $\alpha_{s}$ has minimal scale dependence (conformal window) allows the use of methods based on the gauge-gravity duality [9] to hadronic physics. Its application to nonperturbative QCD calculations will be discussed in Sections 3.9.6 and 4.2 . 


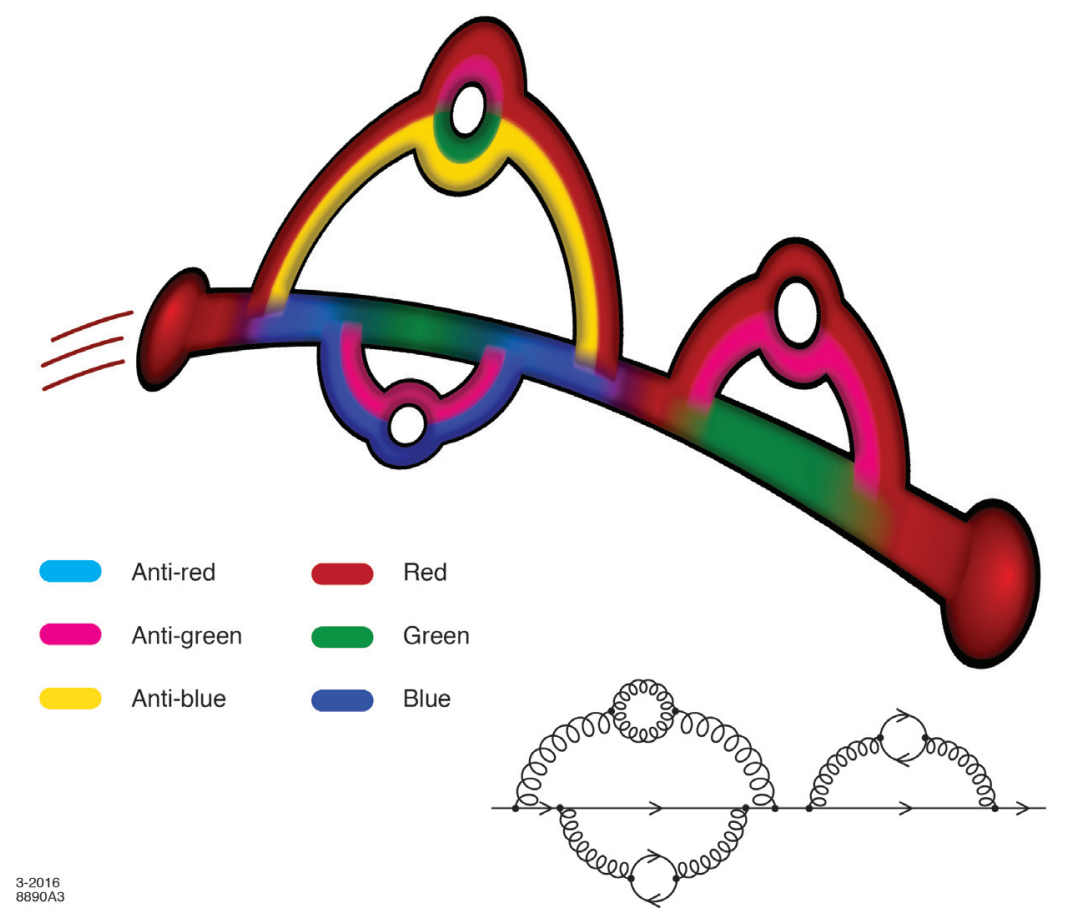

Figure 2.1: (Color online) Color spread and the physics of asymptotic freedom and confinement. In contrast with QED the force carriers of QCD, the gluons, carry color charges which leads to spread of color. The dilution of the initial color charge is responsible for the weakening of the QCD coupling $\alpha_{s}$ at small distances; $i$. e., when the quark experiences a large momentum transfer. Since there is no intrinsic length scale, values of $\alpha_{s}$ at different momentum transfer scales are related by a logarithmic function: $\alpha_{s}$ decreases continuously with increasing momentum transfer (shorter distances) leading to asymptotic freedom. Conversely $\alpha_{s}$ increases logarithmically at larger distances. However, this growth with large distance cannot continue indefinitely since the proton has a finite size: color is confined. Confinement implies that long wavelengths of quarks and gluons are cut off at a typical hadronic size. Consequently, the effects of quantum loops responsible for the logarithmic dependence of $\alpha_{s}$ disappear and $\alpha_{s}$ should freeze to a constant value at hadronic scales $[5,6]$. The evolution of a quark's color is illustrated in the figure. Here, an initially red quark converts to either a green or blue quark when its red color charge is carried away by the emission of a gluon. The anti-red color is symbolized as cyan, anti-green as magenta and anti-blue as yellow. In the accompanying diagram, the straight line represents a color-triplet quark (or an anti-quark), and a curved line represents a color-octet gluon carrying both color and anti-color. 


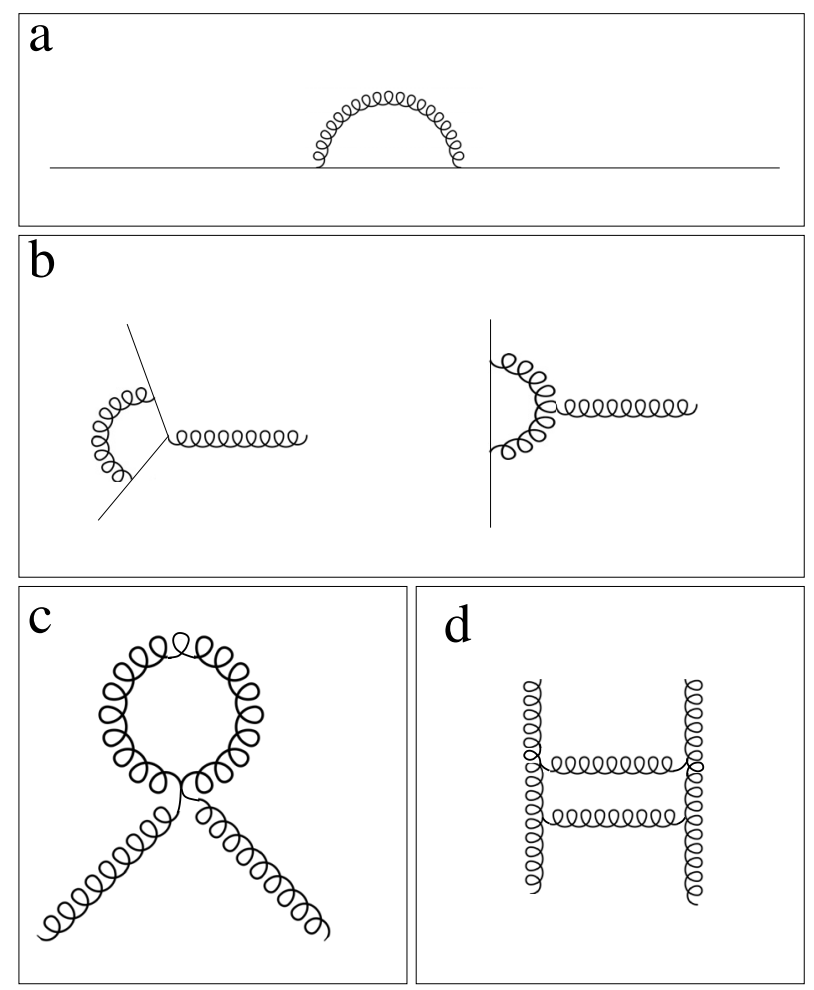

Figure 2.2: Panel a: virtual gluon correction for the quark propagator. Panel b) vertex corrections. Panel c: Tadpole graph. Panel d: H (or ladder) graph.

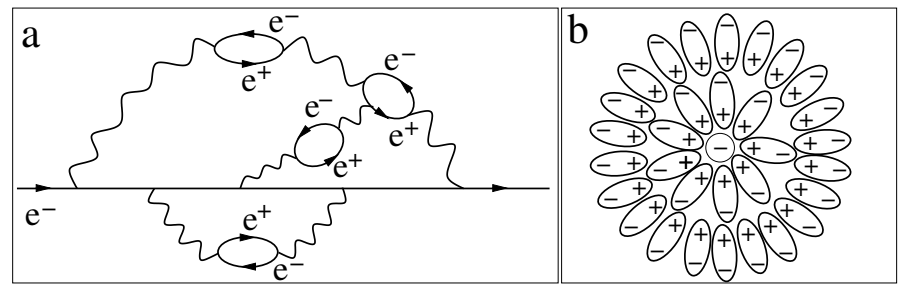

Figure 2.3: Panel a: screening of the QED charge. Panel b: analogy to the charge screening occurring in a dielectric material. 


\section{Chapter 3}

\section{The strong coupling $\alpha_{s}$ in the perturbative domain}

In this chapter, we give a more formal discussion of the strong coupling $\alpha_{s}$ in the perturbative domain. Although the content of this chapter is well explained in textbooks and reviews, we will give a brief review in order to define notations as well as to set the basis for a proper understanding of the behavior of $\alpha_{s}$ in the IR domain, which we postpone until chapter 4 .

We first recall the equations ruling the behavior of $\alpha_{s}$ in the perturbative domain. Then we discuss various techniques which can be used to optimize the perturbative series in order to improve pQCD's predictive power. Finally, we summarize the current experiment/Lattice QCD status on $\alpha_{s}$. The momentum transfer scale at which $\alpha_{s}$ is determined is $Q^{2}=-q^{2}$, with $q$ the four-momentum flow of the process. We have $Q^{2}>0$ for space-like processes. One can work in a given RS, such as the renormalization scheme which follows from dimensional regularization [10]: The minimal-subtraction (MS) renormalization scheme [11] or the $\overline{M S}$ RS [12]. In the $\overline{M S}$ RS one subtracts $\frac{2}{\epsilon}+\log 4 \pi-\gamma_{E}$ (where $\gamma_{E}$ is the Euler constant) after regularizing UV divergent integrals in $4-\epsilon$ dimension. In the MS and $\overline{M S}$ renormalization schemes $\alpha_{s}$ is gauge invariant. It is convenient however, to work in the Landau gauge, $\partial_{\mu} A^{\mu}=0$. Thus, the gauge parameter $\xi$, see e.g., Eq. (3.28), is fixed at $\xi=0$ in this section. 


\subsection{Purpose of the running coupling}

We shall assume that quark masses can be neglected at the typical scale $Q^{2}$, the value characterizing a given reaction. The QCD Lagrangian density then contains no mass or energy scale and is classically conformally invariant:

$$
\mathcal{L}=i \bar{\psi} \gamma^{\mu} D_{\mu} \psi-\frac{1}{4} G_{\mu \nu}^{a} G_{a}^{\mu \nu}
$$

where $\psi$ is the quark field, $G_{\mu \nu}^{a}$ the gluon field strength and $a$ the color index. The gluon field strength is given by $G_{\mu \nu}^{a} \equiv \partial_{\mu} A_{\nu}^{a}-\partial_{\nu} A_{\mu}^{a}+g f^{a b c} A_{\mu}^{b} A_{\nu}^{c}$, with $A_{\mu}^{a}$ the gluon field, $f^{a b c}$ the $\mathrm{SU}(3)$ structure constant and $g$ the dimensionless coupling constant. The bare coupling is $\overline{\alpha_{s}}=g^{2} / 4 \pi$. Although there are no dimensionful parameters in Eq. (3.1), a mass scale $\mu$ is acquired during the renormalization procedure. The emergence of $\mu$ from a Lagrangian without explicit scale is called dimensional transmutation [13]. The value of $\mu$ is arbitrary and is the momentum at which the UV divergences are subtracted. Hence $\mu$ is called the subtraction point or renormalization scale. A dimensionless observable $R\left(Q^{2}, x_{f}\right)$, where $x_{f}$ represents any dimensionless kinematic variables, must be independent of the arbitrary value of $\mu$. The purpose of making $\alpha_{s}$ scale-dependent is to transfer to $\alpha_{s}$ all terms involving $\mu$ in the perturbative series of $R\left(Q^{2}, x_{f}\right)$.

In the perturbative domain, observables are expressed in perturbative expansions of $\overline{\alpha_{s}}:$

$$
R\left(Q^{2}, \mu^{2}, \overline{\alpha_{s}}, x_{f}\right)=\sum_{n=0}^{n_{\max } \sim \pi / \alpha_{s}} r_{n}\left(Q^{2}, \mu^{2}, x_{f}\right)\left(\frac{\overline{\alpha_{s}}}{\pi}\right)^{n} .
$$

Since $R$ is dimensionless and since there is no mass scale in the QCD Lagrangian, the $Q^{2}$ dependence of $\alpha_{s}$ can only be a function of the $Q^{2} / \mu^{2}$ ratio. Except for $r_{0}$ and $r_{1}$ which are independent of $Q^{2} / \mu^{2}$, the coefficients $r_{n}$ are polynomials of $\ln \left(Q^{2} / \mu^{2}\right)$ with highest power $n-1$. The independence of $R$ with respect to $\mu$ is given by the Callan-Symanzik relation for QCD [14]:

$$
\begin{array}{r}
\frac{d}{d \ln \left(\mu^{2}\right)} R\left(Q^{2} / \mu^{2}, \alpha_{s}, x_{f}\right)=\mu^{2} \frac{d}{d \mu^{2}} R\left(Q^{2} / \mu^{2}, \alpha_{s}, x_{f}\right)= \\
\left(\mu^{2} \frac{\partial}{\partial \mu^{2}}+\mu^{2} \frac{\partial \alpha_{s}}{\partial \mu^{2}} \frac{\partial}{\partial \alpha_{s}}\right) R\left(Q^{2} / \mu^{2}, \alpha_{s}, x_{f}\right)=0 .
\end{array}
$$

The $\beta$-function is defined from Eq. (3.3) as $\mu^{2} \frac{\partial \alpha_{s}}{\partial \mu^{2}}=\beta\left(\alpha_{s}\right)$. Setting $t=\ln \left(Q^{2} / \mu^{2}\right)$, Eq. 
(3.3) becomes

$$
\left(-\frac{\partial}{\partial t}+\beta \frac{\partial}{\partial \alpha_{s}}\right) R\left(e^{t}, \alpha_{s}, x_{f}\right)=0 .
$$

We remark that if we had not yet fixed the gauge fixing parameter to $\xi=0$, Eqs. (3.3) and (3.4) would also have a term stemming from the variation of $\xi$. Expressing the observable as a perturbative series now in $\alpha_{s}\left(\mu^{2}\right)$ defined by $\alpha_{s}\left(\mu^{2}\right) \equiv \alpha_{s}$ and $t=$ $\int_{\alpha_{s}}^{\alpha_{s}\left(Q^{2}\right)} \frac{1}{\beta(y)} d y$, one has:

$$
R\left(e^{t}, \alpha_{s}, x_{f}\right)=\sum_{n=0}^{n_{\max } \sim \pi / \alpha_{s}} r_{n}\left(1, \alpha_{s}, x_{f}\right)\left(\frac{\alpha_{s}\left(\mu^{2}\right)}{\pi}\right)^{n}
$$

One can check that it is a solution of Eq. (3.4). Setting $e^{t}=1$, i.e., setting the physical scale $Q$ equal to the renormalization scale $\mu, Q^{2}=\mu^{2}$, makes the coefficients $r_{n}$ independent of $Q^{2} / \mu^{2}$; the $\mu$-dependence has been folded into $\alpha_{s}\left(\mu^{2}\right)$. Choosing $Q^{2}=$ $\mu^{2}$ thus yields the simplest form for the perturbative expansions of given observables. There are however disadvantages to this choice, such as an unintuitive interpretation of the coupling and convergence issues with the series (renormalon problem). Other choices, while leading to more complex perturbative expressions, can be more physically motivated. This will be discussed in Sections 3.7.1 and 3.8. Another choice would be similar to a different choice of RS, see Section 3.7.1 and Fig. 3.4. One generally keeps $Q^{2} \sim \mu^{2}$ so that the coefficients $r_{n}$ remain small enough for the perturbative expansion of $R\left(t, \alpha_{s}, x_{f}\right)$ to be valid.

\subsection{The evolution of $\alpha_{s}$ in perturbative QCD}

The scale dependence of the strong coupling is controlled by the $\beta$-function which can be expressed in the UV as a perturbative series:

$$
Q^{2} \frac{\partial \alpha_{s}}{\partial Q^{2}}=\beta\left(\alpha_{s}\right)=-\left(\frac{\alpha_{s}}{4 \pi}\right)^{2} \sum_{n=0}\left(\frac{\alpha_{s}}{4 \pi}\right)^{n} \beta_{n} .
$$

The values of the first terms of the $\beta$-series are [15]:

$$
\beta_{0}=11-\frac{2}{3} n_{f}
$$


and $[16,17]$

$$
\beta_{1}=102-\frac{38}{3} n_{f}
$$

with $n_{f}$ the number of quark flavors active at the scale $Q^{2}$. These terms are RSindependent (as long as quark masses are neglected, see Section 3.3) because of the UV renormalizability of QCD - only the first two loops are dominated by small distance processes: the 1 and 2-loops integrals diverge in the $Q^{2} \rightarrow \infty$ limit, while higher loops are finite in this limit. The higher order $\beta$-terms are known but are RS-dependent. Since they also encompass nonperturbative contributions not included in the perturbative series Eq. (3.6), there is an inherent ambiguity for these terms. In schemes based on the dimensional regularization method the higher order terms in the minimal-subtraction scheme $\overline{M S}$ (the most common RS used [11, 12]) are [18]

$$
\beta_{2}=\frac{2857}{2}-\frac{5033}{18} n_{f}+\frac{325}{54} n_{f}^{2}
$$

and $[19]$

$$
\begin{aligned}
\beta_{3}=\left(\frac{149753}{6}+3564 \xi(3)\right)-\left(\frac{1078361}{162}\right. & \left.+\frac{6508}{27} \xi(3)\right) n_{f} \\
& +\left(\frac{50065}{162}+\frac{6472}{81} \xi(3)\right) n_{f}^{2}+\frac{1093}{729} n_{f}^{3}
\end{aligned}
$$

with $\xi(3)$ the Apéry constant, $\xi(3) \simeq 1.20206$. Calculations are ongoing for $\beta_{4}[20]$. All the $\beta_{i}$ coefficients are gauge independent in the $\overline{M S}$ scheme [21]. In the momentum space subtraction (MOM) scheme [22, 23] and Landau gauge, these couplings are [24]:

$$
\beta_{2}=3040.48-625.387 n_{f}+19.3833 n_{f}^{2}
$$

and

$$
\beta_{3}=100541-24423.3 n_{f}+1625.4 n_{f}^{2}-27.493 n_{f}^{3} .
$$

They can be found for the minimal MOM scheme and Landau gauge in [25]. (The MOM scheme renormalization condition forces the virtual quark propagator to a free massless propagator form). Some important points must be made: first the $\beta_{i}$ are independent of $\alpha_{s}$; they are expansions in orders of $\hbar$, see Section 3.4. Second, the signs of the $\beta_{i}$ control how $\alpha_{s}$ run. We have $\beta_{0}>0$ for $n_{f} \leq 16, \beta_{1}>0$ for $n_{f} \leq 8, \beta_{2}>0$ for $n_{f} \leq 5$, and $\beta_{3}$ is always positive. Consequently, $\alpha_{s}$ decreases at high momentum transfer, leading to 
the quark asymptotic freedom of pQCD. Finally, the $\beta_{i}$ are sometimes defined with an additional multiplying factor $1 /(4 \pi)^{i+1}$.

The exact analytical solution to Eq. (3.6) is known only to $\beta_{0}$ order. At this order, Eq. (3.6) is:

$$
\frac{Q^{2}}{\alpha_{s}^{2}} \frac{\partial \alpha_{s}}{\partial Q^{2}}=-\frac{1}{4 \pi} \beta_{0},
$$

that is

$$
-\frac{4 \pi d \alpha_{s}}{\beta_{0} \alpha_{s}^{2}}=\frac{d Q^{2}}{Q^{2}} .
$$

Integrating (3.14) between $Q^{2}$ and $\mu_{0}^{2}$ yields:

$$
\frac{4 \pi}{\alpha_{s}\left(\mu_{0}^{2}\right)}-\frac{4 \pi}{\alpha_{s}\left(Q^{2}\right)}=\beta_{0} \ln \left(\frac{\mu_{0}^{2}}{Q^{2}}\right) \text {. }
$$

Eq. (3.15) expresses the general rule that pure pQCD calculations only provide the evolution of an observable relative to the value of this observable given at an arbitrary scale, here $\mu_{0}$. To convey this more conveniently, the QCD scale parameter $\Lambda$ is introduced. At $\beta_{0}$ order, it is defined as:

$$
\Lambda^{2} \equiv \mu^{2} e^{-\frac{4 \pi}{\beta_{0} \alpha_{s}\left(\mu^{2}\right)}}
$$

which yields the familiar 1-loop solution:

$$
\alpha_{s}\left(Q^{2}\right)=\frac{4 \pi}{\beta_{0} \ln \left(Q^{2} / \Lambda^{2}\right)}
$$

We remark that the scale parameter $\Lambda$ is RS-dependent, see Table (3.1). Consequently, although the expressions of $\alpha_{s}$ at order $\beta_{0}$ or $\beta_{1}$ are universal, their numerical values would still depend on the choice of the RS through $\Lambda$, unless the observable approximants in which $\alpha_{s}$ is used are also limited to their RS-independent orders. The relation between $\Lambda_{1}$ in a scheme 1 and $\Lambda_{2}$ in a scheme 2 is, at the one-loop order:

$$
\Lambda_{2}=\Lambda_{1} e^{\frac{2 v_{1}}{\beta_{0}}}
$$

where $v_{1}$ is the leading order difference between the $\alpha_{s}\left(Q^{2}\right)$ in the two RS: $\alpha_{s}^{(2)}\left(Q^{2}\right)=$ $\alpha_{s}^{(1)}\left(Q^{2}\right)\left[1+v_{1} \alpha_{s}^{(1)}\left(Q^{2}\right) / \pi\right]$. For example, the $\overline{M S}$ and MOM scale parameters are related by:

$$
\Lambda_{M O M}=\Lambda_{\overline{M S}} e^{\frac{507-40 n_{f}}{792-32 n_{f} / 3}}
$$

so that $\Lambda_{M O M}=1.847 \Lambda_{\overline{M S}}$ for $n_{f}=2, \Lambda_{M O M}=1.817 \Lambda_{\overline{M S}}$ for $n_{f}=3$ and $\Lambda_{M O M}=$ 
$1.783 \Lambda_{\overline{M S}}$ for $n_{f}=4$.

The value of $\Lambda$ is also dependent on the number of active quark flavors, see Section 3.3 .

An exact analytical relation corresponding to Eq. (3.15) also exists at order $\beta_{1}$ :

$$
\frac{4 \pi}{\alpha_{s}\left(Q^{2}\right)}-\frac{\beta_{1}}{\beta_{0}} \ln \left(\frac{4 \pi}{\alpha_{s}\left(Q^{2}\right)}+\frac{\beta_{1}}{\beta_{0}}\right)=\frac{4 \pi}{\alpha_{s}\left(\mu^{2}\right)}-\frac{\beta_{1}}{\beta_{0}} \ln \left(\frac{4 \pi}{\alpha_{s}\left(\mu^{2}\right)}+\frac{\beta_{1}}{\beta_{0}}\right)+\beta_{0} \ln \left(\frac{Q^{2}}{\mu^{2}}\right),
$$

and an exact, but non-analytical, $\alpha_{s}$ solution is given by [26]:

$$
\alpha_{s}\left(Q^{2}\right)=-\frac{4 \pi \beta_{0}}{\beta_{1}} \frac{1}{W_{-1}(z)},
$$

with $z \equiv-\frac{\beta_{0}^{2}}{e \beta_{1}}\left(\frac{\Lambda^{2}}{Q^{2}}\right)^{\beta_{0}^{2} / \beta_{1}}$ and $W_{-1}(z)$ is the lower branch of the real-valued Lambert function solution of the equation $z=W(z) e^{W(z)}$. An accurate approximation of $W_{-1}$ can be found in [27]. The scale parameter at $\beta_{1}$ order is approximately (see Eq. (3.24) truncated at $\beta_{1}$ ):

$$
\Lambda^{2}=Q^{2}\left(\frac{4 \pi}{\beta_{0} \alpha_{s}\left(Q^{2}\right)}+\frac{\beta_{1}}{4 \pi \beta_{0}}\right)^{\frac{\beta_{1}}{4 \pi \beta_{0}}} e^{-\frac{4 \pi}{\beta_{0} \alpha_{s}\left(Q^{2}\right)}}
$$

Extending this approach to $\beta_{2}$ is difficult but the authors of [26] provided an approximate solution:

$$
\alpha_{s}\left(Q^{2}\right)=-\frac{4 \pi \beta_{0}}{\beta_{1}} \frac{1}{1-\frac{\beta_{2} \beta_{0}}{\beta_{1}^{2}} W_{-1}(z)},
$$

with $z \equiv-\frac{\beta_{0}^{2}}{\beta_{1}}\left(\frac{\Lambda^{2}}{Q^{2}}\right)^{\beta_{0}^{2} / \beta_{1}} e^{\frac{\beta_{2} \beta_{0}}{\beta_{1}^{2}}-1}$. The exact and approximate $\alpha_{s}$ at order $\beta_{1}$ and $\beta_{2}$, respectively, are shown in Fig. 3.1.

For orders up to $\beta_{3}$, an approximate analytical solution to Eq. (3.6) is obtained by 


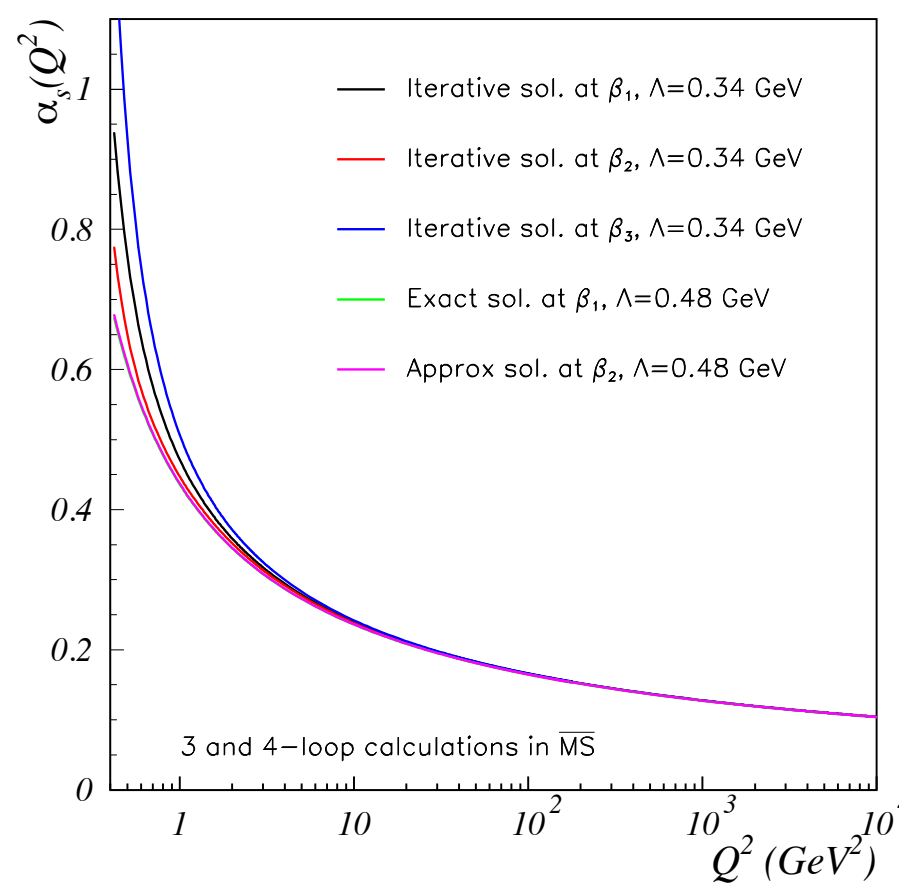

Figure 3.1: The perturbative coupling $\alpha_{s}\left(Q^{2}\right)$ computed at different orders in $\beta_{i}$ using the iterative method (black, red and blue lines: Eq. (3.24) at orders $\beta_{1}, \beta_{2}$ and $\beta_{3}$, respectively) and the exact and approximate solutions, Eq. (3.21) ( $\beta_{1}$, green line) and Eq. (3.23) $\left(\beta_{2}\right.$, magenta line), respectively. The green and magenta lines are very close to each other. We use $n_{f}=3$ and $\Lambda=0.34 \mathrm{GeV}$ for the iterative method and adjusted $\Lambda$ to $0.48 \mathrm{GeV}$ for the others so that all calculations match at large $Q^{2}$. The coefficients $\beta_{2}$ and $\beta_{3}$ are calculated in the $\overline{M S}$ renormalization scheme.

an iterative method [28]:

$$
\begin{aligned}
\alpha_{s}\left(Q^{2}\right)= & \frac{4 \pi}{\beta_{0} \ln \left(Q^{2} / \Lambda^{2}\right)}\left[1-\frac{\beta_{1}}{\beta_{0}^{2}} \frac{\ln \left[\ln \left(Q^{2} / \Lambda^{2}\right)\right]}{\ln \left(Q^{2} / \Lambda^{2}\right)}\right. \\
+ & \frac{\beta_{1}^{2}}{\beta_{0}^{4} \ln ^{2}\left(Q^{2} / \Lambda^{2}\right)}\left(\left(\ln \left[\ln \left(Q^{2} / \Lambda^{2}\right)\right]\right)^{2}-\ln \left[\ln \left(Q^{2} / \Lambda^{2}\right)\right]-1+\frac{\beta_{2} \beta_{0}}{\beta_{1}^{2}}\right) \\
& +\frac{\beta_{1}^{3}}{\beta_{0}^{6} \ln ^{3}\left(Q^{2} / \Lambda^{2}\right)}\left(-\left(\ln \left[\ln \left(Q^{2} / \Lambda^{2}\right)\right]\right)^{3}+\frac{5}{2}\left(\ln \left[\ln \left(Q^{2} / \Lambda^{2}\right)\right]\right)^{2}\right. \\
& \left.+2 \ln \left[\ln \left(Q^{2} / \Lambda^{2}\right)\right]-\frac{1}{2}-3 \frac{\beta_{2} \beta_{0}}{\beta_{1}^{2}} \ln \left[\ln \left(Q^{2} / \Lambda^{2}\right)\right]+\frac{\beta_{3} \beta_{0}^{2}}{2 \beta_{1}^{3}}\right) \\
& \left.+\mathcal{O}\left(\frac{\left.\ln \left[\ln \left(Q^{2} / \Lambda^{2}\right)\right]\right)^{4}}{\ln \left(Q^{2} / \Lambda^{2}\right)}\right)\right] .
\end{aligned}
$$

The integration constant $\Lambda$ is the only unknown parameter in the monotonic equation 
(3.24). Consequently, at a given order and in a given RS, the perturbative coupling $\alpha_{s}\left(Q^{2}\right)$ can be fully characterized either by giving the value of $\alpha_{s}$ at a conventional scale, usually, $Q^{2}=M_{Z}^{2}$, or by giving the value of $\Lambda$.

From Eq. (3.24), $\alpha_{s}$ can be extracted from an observable at a given scale, evolved to the conventional scale $M_{Z}^{2}$ and compared to $\alpha_{s}$ extracted using other observables. The agreement of the values, see Section 3.9, demonstrates the universality of $\alpha_{s}$, the validity of Eq. (3.6), and constitutes an important consistency check of QCD. To determine $\alpha_{s}\left(M_{Z}^{2}\right)$, or equivalently $\Lambda$, actual measurements or nonperturbative calculations are necessary, see Section 3.9.

\subsection{Quark thresholds}

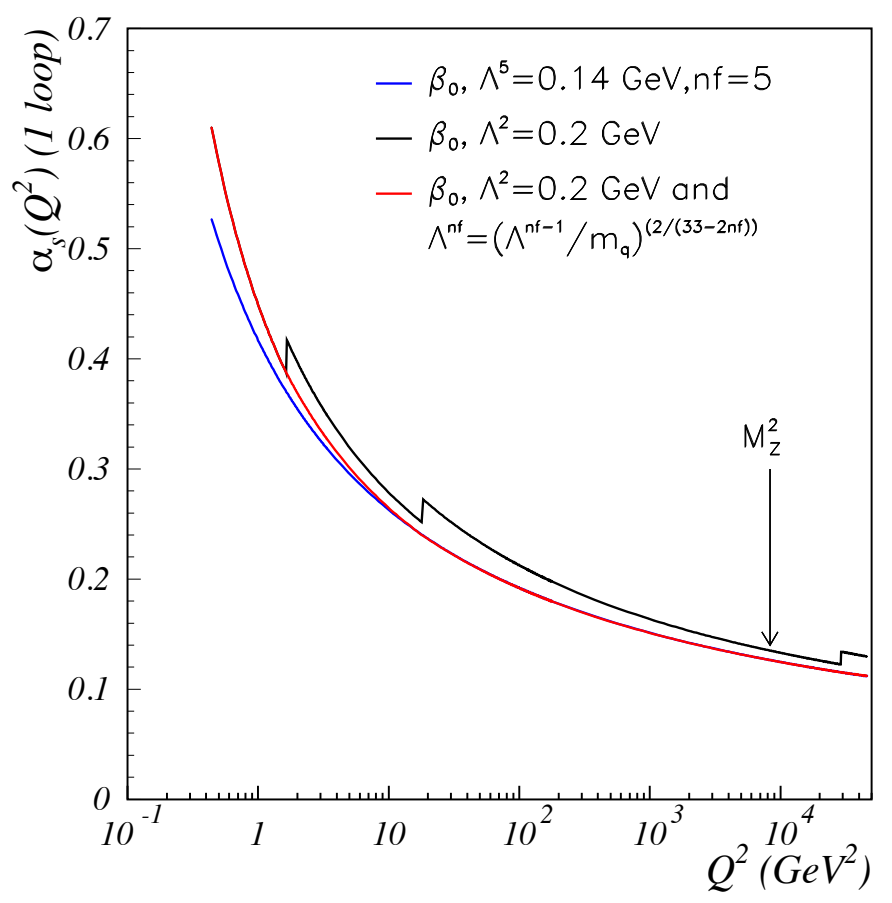

Figure 3.2: The running coupling $\alpha_{s}\left(Q^{2}\right)$ at order $\beta_{0}$ for a constant $n_{f}=5$ (blue line), with $\Lambda^{n_{f}=5}$ chosen so that it matches the experimental coupling value at $Q^{2}=M_{Z}^{2}$ (shown by the arrow). The effect of quarks becoming active is shown by the black line. The red line corrects for it by threshold matching. We adjusted $\Lambda^{n_{f}=2}$ to match the data at $Q^{2}=M_{Z}^{2}$.

Quark masses create additional higher order perturbation terms that have, for light quarks, a small influence. However, they do indirectly and importantly affect $\alpha_{s}$ through $n_{f}$, the number of active quark flavors at scale $Q^{2}$. A quark flavor is active if its mass 
$m_{q}^{2} \ll Q^{2}$. For all other purposes, $m_{q}$ can be set to zero regardless whether it is active or not. The loose requirement $m_{q} \ll Q$ implies that $\alpha_{s}$ varies smoothly when passing a quark threshold, rather than in discrete steps as depicted in Fig. 3.2. The matching of the values of $\alpha_{s}$ below and above a quark threshold makes $\Lambda$ to depend on $n_{f}$. For example, at leading order, requesting that

$$
\alpha_{s}^{n_{f}-1}\left(Q^{2}=m_{q}^{2}\right)=\alpha_{s}^{n_{f}}\left(Q^{2}=m_{q}^{2}\right)
$$

imposes:

$$
\Lambda^{n_{f}}=\Lambda^{n_{f}-1}\left(\frac{\Lambda^{n_{f}-1}}{m_{q}}\right)^{2 /\left(33-2 n_{f}\right)} .
$$

The formula at next order, $\beta_{1}$, can be found in [29]. The four-loop matching in the $\overline{M S}$ $\mathrm{RS}$ is given in [28]. Another procedure to treat the variation of active $n_{f}$ keeps the quark masses nonzero in the quark loops and treat $n_{f}$ analytically [30].

\subsection{Computation of the pQCD effective coupling}

In this section, we illustrate how the coefficients $\beta_{i}$ of the perturbative $\beta$-series are calculated by giving the steps leading to the expression of $\alpha_{s}$ to lowest order and thus of $\beta_{0}$. This allows us to understand the physical origin of the processes contributing to the running of the coupling in the UV domain. The $M S$ scheme is used and we neglect quark masses.

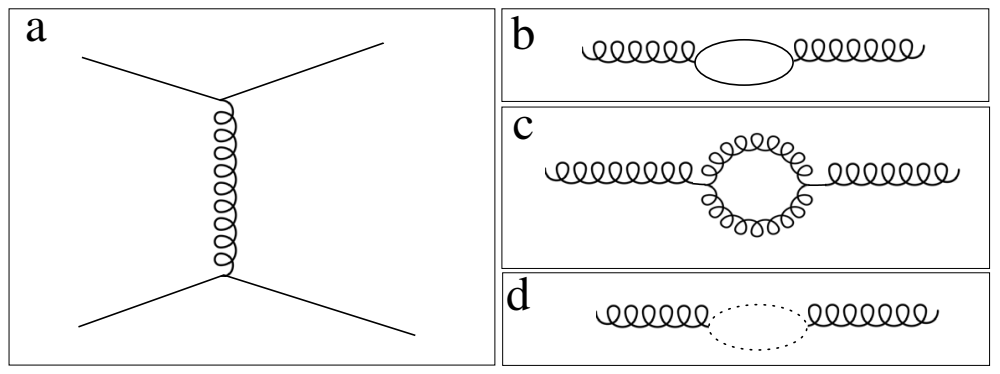

Figure 3.3: Panel a: leading order (Born) contribution to quark-quark scattering. Panels b, c and d: quark, gluon and ghost loop corrections to the gluon propagator, respectively.

As already discussed, $\alpha_{s}\left(Q^{2}\right)$ can be defined using the potential approach. The higher-order processes producing a deviation of the quark-quark scattering amplitude from the leading zero-order (Born amplitude, see Fig. 3.3a) are folded into the bare coupling, thereby defining the effective coupling. The processes contributing to next 
order -first order- are shown in Figs. 2.2a, b and c, and 3.3b, c and d. We first consider the effect of quark loops on the gluon propagator, see Fig. 3.3 b. Applying QCD's Feynman rules yields:

$$
D_{q}^{\mu \nu}(q)=\frac{\overline{\alpha_{s}}}{3 \pi}\left(q^{\mu} q^{\nu}-\eta^{\mu \nu} q^{2}\right) \ln \left(\frac{Q^{2}}{\mu^{2}}\right) n_{f} \frac{\delta_{a b}}{2},
$$

where $\overline{\alpha_{s}}$ is the bare coupling constant and $\mu^{2}$ the subtraction point. The term in Eq. (3.27) is analogous to the one causing the QED effective coupling to run. In addition, in QCD, gluon loops (Fig. 3.3c) and tadpole graphs (Fig. 2.2c) may also contribute. In the $M S$ scheme, however, tadpoles are suppressed. The calculation of the gluon loop term is similar to the quark loop one and yields:

$$
D_{g}^{\mu \nu}(q)=\frac{3 \overline{\alpha_{s}}}{4 \pi} \delta_{a b}\left[\frac{11}{6} q^{\mu} q^{\nu}-\frac{19}{12} \eta^{\mu \nu} q^{2}+\frac{1-\xi}{2}\left(q^{\mu} q^{\nu}-\eta^{\mu \nu} q^{2}\right)\right] \ln \left(\frac{Q^{2}}{\mu^{2}}\right)
$$

with $\xi$ a gauge fixing term. Contrary to the quark loop contribution, the propagator $D_{g}^{\mu \nu}(q)$ is not proportional to $\left(q^{\mu} q^{\nu}-\eta^{\mu \nu} q^{2}\right)$, i.e., when gluon loops are accounted for, the gluon propagator is not transverse anymore and current conservation is violated: $D_{g}^{\mu \nu} q_{\mu} \neq 0$. There are several ways to address this. Gauges where gluons are always transverse, such as the axial gauge or the light-cone gauge, can be chosen. Alternatively, fictitious particles of spin zero but obeying the Fermi-Dirac statistics can be introduced, the Fadeev-Popov ghosts [31]. Their characteristics are chosen so that they add a loop contribution to the gluon propagator, see Fig. 3.3d, that complements the non-transverse term in Eq. (3.28), to make it transverse. They contribute as:

$$
D_{g h}^{\mu \nu}(q)=-\frac{3 \overline{\alpha_{s}}}{4 \pi} \delta_{a b}\left[\frac{1}{6} q^{\mu} q^{\nu}+\frac{1}{12} \eta^{\mu \nu} q^{2}\right] \ln \left(\frac{Q^{2}}{\mu^{2}}\right)
$$

Contrary to QED, in QCD the fermion self-energy (Fig. 2.2a) and vertex corrections (Fig. 2.2b) do not cancel each other and need to be accounted for. At first order the self-energy correction is:

$$
G_{q}(p)=\frac{\not p}{p^{2}} \delta_{a b}\left[1-\xi \frac{\overline{\alpha_{s}}}{3 \pi} \ln \left(\frac{-p^{2}}{\mu^{2}}\right)\right]
$$


and the vertex correction yields:

$$
\Gamma_{\mu}^{\alpha \beta ; a}(q)=-i \sqrt{4 \pi \overline{\alpha_{s}}} \frac{\lambda_{\alpha \beta}^{a}}{2} \gamma_{\mu}\left[1-\frac{\overline{\alpha_{s}}}{4 \pi} \ln \left(\frac{Q^{2}}{\mu^{2}}\right)\left\{\frac{4}{3} \xi+3\left(1-\frac{1-\xi}{4}\right)\right\}\right]
$$

with $\lambda_{\alpha \beta}^{a}$ the Gell-Mann matrices, $a$ the gluon color index and $\alpha, \beta$ the color indices for the two quark lines.

In renormalization schemes based on dimensional regularization, the sum of all the amplitudes is gauge invariant. Summing them yields the quark-quark scattering amplitude up to next-to-leading order (NLO)

$$
\mathcal{M}=\mathcal{M}_{\text {Born }}\left[1-\frac{\overline{\alpha_{s}}}{4 \pi} \ln \left(\frac{Q^{2}}{\mu^{2}}\right)\left\{\frac{2 n_{f}}{3}-\frac{13}{2}-\frac{9}{2}\right\}\right] \text {. }
$$

One can trace back the $2 n_{f} / 3$ term to the quark loop corrections to the gluon propagator, the $-13 / 2$ term to the gluon and ghost loop corrections to the gluon propagator, and the $-9 / 2$ term to the vertex corrections. (The remaining graph -the quark self-energy, Fig. 2.2a- does not contribute in the Landau gauge).

Folding the deviations from the Born term into the coupling constant defines the effective coupling $\alpha_{s}$, which is then at one loop:

$$
\alpha_{s}\left(Q^{2}\right)=\alpha_{s}\left(\mu^{2}\right)\left[1-\frac{\alpha_{s}\left(\mu^{2}\right)}{12 \pi} \ln \left(\frac{Q^{2}}{\mu^{2}}\right)\left\{2 n_{f}-33\right\}\right] .
$$

Inverting the expression and assuming that $\alpha_{s}\left(\mu^{2}\right)$ is small for $\mu^{2} \gg Q^{2}$, yields:

$$
\frac{4 \pi}{\alpha_{s}\left(Q^{2}\right)}=\frac{4 \pi}{\alpha_{s}\left(\mu^{2}\right)}+\frac{33-2 n_{f}}{3} \ln \left(\frac{Q^{2}}{\mu^{2}}\right) \text {. }
$$

Using Eq. (3.15), we identify

$$
\beta_{0}=11-\frac{2 n_{f}}{3}
$$

In $\mathrm{QED}$, only the photon propagator contributes to the running of the coupling constant since Ward-Takahashi identities guarantee that the photon bremsstrahlung cancels the vertex corrections. However, the Ward-Takahashi identities do not hold for non-Abelian theories. A more restricted constraint is given by the Slavnov-Taylor identities [32], which ensure that intermediate gauge-dependent quantities used in calculations, such as Green's functions, yield final gauge-independent results for observables. These constraints lead to relations between the various Green's functions characterizing 
the propagation and interaction amplitudes and, as such, are especially important for solving the Schwinger-Dyson equations, see Section 4.4 .

\subsection{Renormalization group}

In this paragraph, the renormalization group $(\mathrm{RG})$ approach is discussed. It provides a more formal view of the mechanism inducing the running of $\alpha_{s}$. This more formal derivation of $\alpha_{s}\left(Q^{2}\right)$ is useful to understand how it is defined in some of the lattice and Schwinger-Dyson studies in the IR, see Sections 4.4 and 4.5.

To regulate divergences, renormalization factors $Z_{i}\left(Q^{2}\right)$ are introduced to define renormalized fields:

$$
A_{a, \mu}=Z_{3}^{1 / 2}\left(Q^{2}\right) A_{a, \mu}^{R}\left(Q^{2}\right), \quad \psi=Z_{2}^{1 / 2}\left(Q^{2}\right) \psi^{R}\left(Q^{2}\right), \quad C_{a}=\widetilde{Z}_{3}^{1 / 2}\left(Q^{2}\right) C_{a}^{R}\left(Q^{2}\right)
$$

where the superscript $R$ indicates the now scale-dependent renormalized fields, and $C_{a}$ is the ghost field. $Z_{3}$ is the gluon propagator renormalization factor, $Z_{2}$ is the quark self-energy renormalization factor and $\widetilde{Z}_{3}$ is the ghost propagator renormalization factor. The ghost propagator is obtained by definition from the inverse of the Fadeev-Popov operator $(-\partial+A) A$. The coupling constant is likewise renormalized: $\overline{\alpha_{s}}=Q^{2 \varepsilon} Z_{\alpha}\left(Q^{2}\right) \alpha_{s}\left(Q^{2}\right)$ where $\varepsilon$ is the dimensional parameter in the $M S$ scheme. In such space, integrals are carried out in $4-2 \varepsilon$ dimensions and the UV divergences are regularized to $1 / \varepsilon$ poles. The $Z_{i}$ are constructed as functions of $1 / \varepsilon$, such that they cancel the $1 / \varepsilon$ poles. Since $\overline{\alpha_{s}}=Q^{2 \varepsilon} Z_{\alpha}\left(Q^{2}\right) \alpha_{s}\left(Q^{2}\right)=\mu^{2 \varepsilon} Z_{\alpha}\left(\mu^{2}\right) \alpha_{s}\left(\mu^{2}\right), Z_{\alpha}$ defines the scale dependence of the effective coupling such that $\alpha_{s}\left(Q^{2}\right)=\mathcal{Z}_{\alpha}\left(Q^{2}, \mu^{2}\right) \alpha_{s}\left(\mu^{2}\right)$, with $\mathcal{Z}_{\alpha}\left(Q^{2}, \mu^{2}\right) \equiv\left(\mu^{2 \varepsilon} / Q^{2 \varepsilon}\right)\left[Z_{\alpha}\left(Q^{2}\right) / Z_{\alpha}\left(\mu^{2}\right)\right]$. The $\mathcal{Z}_{\alpha}$ form a group with a composition law: $\mathcal{Z}_{\alpha}\left(Q^{2}, \mu^{2}\right)=\mathcal{Z}_{\alpha}\left(Q^{2}, \mu_{0}^{2}\right) \mathcal{Z}_{\alpha}\left(\mu_{0}^{2}, \mu^{2}\right)$, a unity element: $\mathcal{Z}_{\alpha}\left(Q^{2}, Q^{2}\right)=1$ and an inversion law: $\mathcal{Z}_{\alpha}\left(Q^{2}, \mu^{2}\right)=\mathcal{Z}_{\alpha}^{-1}\left(\mu^{2}, Q^{2}\right)$. The invariance under the infinitesimal transformation $Q \rightarrow Q+\delta Q$ leads to the RG Eq. (3.6) with the leading order relation

$$
Z_{\alpha}\left(Q^{2}\right)=1-\frac{\beta_{0} \alpha_{s}\left(Q^{2}\right)}{4 \pi \varepsilon}
$$

necessary to cancel the $1 / \varepsilon$ pole. This relation defines the coefficient $\beta_{0}$.

In addition to field and coupling renormalization factors, vertex renormalization factors can also be introduced. They are straightforwardly related to the $Z_{i}$ already introduced. For example, the quark-quark-gluon vertex renormalization factor $Z_{1}$ is 
$Z_{1}=\left(Z_{2} Z_{\alpha} Z_{3} Z_{2}\right)^{1 / 2}$. From this, $Z_{\alpha}$ can be extracted:

$$
Z_{\alpha}=\frac{Z_{1}^{2}}{Z_{2}^{2} Z_{3}}
$$

Clearly, $Z_{\alpha}$ can also be obtained from other graphs, such as the ghost-gluon vertex. In the $M S$ scheme, the Feynman rules yield at NLO $Z_{1}=1-\alpha_{s}\left(Q^{2}\right)(3+4 / 3) / 4 \pi \varepsilon$, $Z_{2}=1-\alpha_{s}\left(Q^{2}\right)(4 / 3) / 4 \pi \varepsilon$ and $Z_{3}=1-\alpha_{s}\left(Q^{2}\right)\left(-5+2 n_{f} / 3\right) / 4 \pi \varepsilon$. Injecting these expressions in Eq. (3.38), assuming that $\alpha_{s}\left(Q^{2}\right)$ is small, and identifying with Eq. (3.37) yields:

$$
\beta_{0}=11-\frac{2 n_{f}}{3}
$$

\subsection{The Landau pole and the QCD parameter $\Lambda$}

The Landau pole is the point where the perturbative expression of $\alpha_{s}$, Eq. (3.24), diverges. It occurs at the value of the scale parameter $\Lambda$. As we shall discuss, the divergence of the perturbative prediction for the running coupling does not usually appear in a nonperturbative approach such as AdS/QCD.

The value of $\Lambda$ in pQCD depends on the RS, on the order of the $\beta$-series, $\beta_{i}$, on the number of flavors $n_{f}$, and on the approximation chosen to solve Eq. (3.6) at orders higher than $\beta_{1}$. At order $\beta_{0}$, the Landau singularity is a simple pole whereas at higher order, it acquires a more complicated structure. The pole is located on the positive real axis of the complex $Q^{2}$-plane, and is thus unphysical: a pole at $\Lambda<0$ would correspond to production of on-shell particles. If $\Lambda>0$, a physical pole would correspond to causality-

violating tachyons. The existence of the Landau pole thus implies that the perturbative expression of $\alpha_{s}$ is a non-observable quantity.

The appearance of an unphysical pole at $\Lambda$ characterizes the scale at which pQCD breaks down; i.e., where $\alpha_{s}$ becomes large. Consequently, the value of $\Lambda$ is often associated with the confinement scale, or equivalently the hadronic mass scale. An explicit relation between hadron masses and $\Lambda$ is known in the framework of holographic QCD; see Sec. 3.9.6. $\Lambda$ can also be related to nonperturbative terms in the context of the analytic QCD approach, see Section 5.3.

We remark that Landau poles were initially identified in the context of Abelian QED. However, its value, $\Lambda \sim 10^{30-40} \mathrm{GeV}$, is well above the Planck scale [33]; it is thus expected that new physics would suppress the occurrence of this unphysical divergence. 


\subsection{Improvement of the perturbative series}

Naively, one may think that at leading order, the smaller the value of $\Lambda$; i.e., the smaller the momentum scale at which the Landau pole divergence occurs, the slower the increase of $\alpha_{s}\left(Q^{2}\right)$ as $Q^{2}$ decreases. This would imply that for expansions at moderate momentum scale, renormalization schemes with small $\Lambda$ are preferable. Approximate values of $\Lambda$ in different schemes are given in Table 3.1. However, such a simple criterium is foiled by the renormalon growth of the coefficients of the perturbative series. Different growths in different renormalization schemes may balance the difference in values of $\Lambda$. In principle, at large enough order, the balancing should be nearly exact since the observable described is RS-independent. Some renormalization schemes have inherently fast rising coefficients, independently of the renormalon problem, see e.g., Ref. [34] in which it is shown that even for perturbative series tailored to be free of renormalons, the $\overline{M S}$ scheme leads to intrinsically fast growing coefficients. The relations between RS and their associated $\Lambda$ are discussed in Ref. [22].

\begin{tabular}{|c|c|c|c|c|c|c|c|}
\hline RS/eff. charge & $M S$ & $M S$ & $M O M$ & $V$ & $g_{1}$ & $R$ & $\tau$ \\
\hline \hline$\Lambda(\mathrm{GeV})$ & 0.34 & 0.30 & 0.62 & 0.48 & 0.92 & 0.48 & 1.10 \\
\hline
\end{tabular}

Table 3.1: Examples of approximate values of $\Lambda$ in different renormalization schemes or effective charge definitions, for $n_{f}=3$.

In this section, we shall discuss several ways to improve perturbative series. A principle of the renormalization group is that a prediction for an observable cannot depend on a theoretical convention such as the choice of the renormalization scheme. The optimization of perturbative series can thus be tied to minimizing its RS-dependence since it brings the pQCD approximant closer to the RS-independent observable. Such an optimization and minimization of the RS-dependence clearly increases the predictive power of pQCD.

\subsubsection{Effective charges and commensurate scale relations}

It was proposed by Grunberg that the QCD coupling can be defined directly from any experimental observable [4] which is predictable in PQCD. In such a case, the coupling is called an "effective charge". The terminology "effective charges" is sometimes used with different definitions. We will use it here in the sense defined by Grunberg.

The definition of the coupling from an observable obeys the RG Eq. (3.6) and has important advantages: an effective charge is RS-independent, free of divergences and 
analytic when crossing quark thresholds; it improves perturbative expansions (suppressing in particular the renormalon problem), and its definition is extendible to the IR nonperturbative domain. It also unifies QED and QCD coupling's definitions. In the case of QED, the running coupling $\alpha\left(q^{2}\right)$ was defined by Gell-Mann and Low from the static Coulomb potential [35]; i.e., the strength of the Coulomb interaction of heavy test charges at momentum transfer $t=q^{2}$. The apparent disadvantage of Grunberg's effective charge approach is a process-dependence of the coupling. However, different effective charges obtained from different observables can be analytically related to each other as we will see shortly.

The general prescription to form Grunberg's effective charge is to truncate the perturbative series of an observable to its first term in $\alpha_{s}$. Since this term is RS-independent, so is the effective charge. For example, in the $\overline{M S}$ RS, the Bjorken sum [36] is known in the perturbative domain, up to $\alpha_{\overline{M S}}^{4}[37]$,

$$
\begin{aligned}
\int_{0}^{1^{-}} d x_{f}\left(g_{1}^{p}\left(x_{f}, Q^{2}\right)\right. & \left.-g_{1}^{n}\left(x_{f}, Q^{2}\right)\right)=\frac{g_{A}}{6}\left[1-\frac{\alpha_{\overline{M S}}}{\pi}-3.58\left(\frac{\alpha_{\overline{M S}}}{\pi}\right)^{2}\right. \\
& \left.-20.21\left(\frac{\alpha_{\overline{M S}}}{\pi}\right)^{3}+175.7\left(\frac{\alpha_{\overline{M S}}}{\pi}\right)^{4}+\mathcal{O}\left(\alpha_{\overline{M S}}^{5}\right)\right]
\end{aligned}
$$

where $\alpha_{\overline{M S}}$ is the coupling in the $\overline{M S} \mathrm{RS}$. The effective charge $\alpha_{g_{1}}\left(Q^{2}\right)$ is defined from the truncation:

$$
\int_{0}^{1^{-}} d x_{f}\left(g_{1}^{p}\left(x_{f}, Q^{2}\right)-g_{1}^{n}\left(x_{f}, Q^{2}\right)\right) \equiv \frac{g_{A}}{6}\left[1-\frac{\alpha_{g_{1}}\left(Q^{2}\right)}{\pi}\right] .
$$

In these equations, $g_{1}^{p}$ and $g_{1}^{n}$ are the spin-dependent proton and neutron structure functions, respectively, $g_{A}$ is the nucleon flavor-singlet axial charge, $x_{f}$ is the Bjorken scaling variable. By definition, the integration excludes the $x_{f}=1$ elastic contribution, (which in any case is negligible at large $Q^{2}$ ). Such a definition amounts to a particular choice of RS: In Eq. (3.6), the $\beta_{n}$ are RS-dependent for $n \geq 2$, hence arbitrary, and one is free to define them so that Eq. (3.41) is realized. In a sense, this definition generalizes the introduction of effective running discussed in 2: all small distance quantum effects generating the higher order terms in Eq. (3.40) are folded into the definition of $\alpha_{g_{1}}$. Another effective charge is $\alpha_{D}\left(Q^{2}\right)$, defined from the Adler function [38], a spacelike continuation of $R_{e^{+} e^{-}}(s)$. Another example of an effective charge can be found in Ref. [39] where the observable is defined from the hadronic decay rate of the $\tau$ lepton.

Clearly, effective charges depend on the choice of the observable. A natural conven- 
tional choice would be to follow QED and use $\alpha_{V}$, the strong coupling defined from the static heavy quark potential $[7,40]$. At 3 -loop order and in the $\overline{M S}$ scheme it is:

$$
V\left(Q^{2}\right)=-\frac{1}{2 \pi^{2}} \frac{4}{3} \frac{\alpha_{\overline{M S}}\left[1+a_{1}\left(\frac{\alpha_{\overline{M S}}}{4 \pi}\right)+a_{2}\left(\frac{\alpha_{\overline{M S}}}{4 \pi}\right)^{2}+a_{3}\left(\frac{\alpha_{\overline{M S}}}{4 \pi}\right)^{3}\right]}{Q^{2}}
$$

with $a_{1}=31 / 3-10 n_{f} / 9, a_{2}=456.75-66.35 n_{f}+1.23 n_{f}^{2}$ and $a_{3}=13432.6-3289.91 n_{f}+$ $185.99 n_{f}^{2}-1.37174 n_{f}^{3}$. Numerically, the $a_{i}$ coefficients are $a_{1}=5.88, a_{2}=211.03$ and $a_{3}=3161.00$ for $n_{f}=4$, displaying a typical factorial renormalon growth. The application of this formula to the spectroscopy and decays of $\mathrm{Q}-\overline{\mathrm{Q}}$ systems can be found in Ref. [41]. The one-loop corrections to $\alpha_{V}$ were performed in the late 1970s and early 1980s [7, 42]. The two-loop calculations became available in the late 1990s and early 2000s [43, 44]. Three-loop calculations were made available recently, see [45, 46, 47] and the recent reviews [48, 49]. Alternatively, the determination of $\alpha_{V}$ from its $\overline{M S}$ (or other) RS can be done using Commensurate Scale Relations - to be discussed nextwhich eliminate the renormalon growth problem of the high order coefficients and thus improves the convergence of the pQCD series.

However, the definition of $\alpha_{V}$ as an effective charge must be made with care in a nonAbelian theory. In QED the Gell-Mann-Low coupling effective charge $\alpha\left(q^{2}\right)$ is defined from the momentum space potential, $V\left(q^{2}\right)=\left[e_{1} e_{2} \alpha\left(q^{2}\right)\right] / q^{2}$, which generates the singlephoton exchange scattering amplitude proportional to the charges of two infinitely heavy test charges $e_{1}$ and $e_{2}$. In contrast to its QED equivalent, in QCD multi-gluon exchange between the two static quarks cannot be separated from single-gluon exchange due to the contributions of multi-gluon diagrams. The exchanged gluons become connected by gluon exchange by the 3-gluon and 4-gluon couplings -the " $\mathrm{H}$ " diagrams, see Fig. 2.2d. In QED, the analogous multi-photon exchange diagrams, such as those due to light-bylight scattering, can be suppressed by taking the formal limit of small external charges $e_{1}$ and $e_{2}$. In pQCD, the multi-gluon exchange contributions are infrared divergent $[7,8]$ as the test static quarks are separated; each additional horizontal rung that connects the two vertical gluons contributes an additional IR divergence. Thus a conventional effective charge cannot be directly defined from the heavy quark scattering potential since it should be finite in the IR. The IR divergence can be regularized by a parameter $\mu^{-1}$ which cuts off the loop integration at large distances. For example, if one evaluates the QCD interaction between two heavy quarks in a quarkonium bound state, the value of $\mu$ corresponds to the (finite) size of the bound $\mathrm{Q}-\overline{\mathrm{Q}}$ system considered. The divergence occurs for $\mu=0$. Hence, the effective charge $\alpha_{V}$ depends not only on the specific QCD 
process from which it is defined - as any effective charge does - but it also depends on the hadron environment in which this process occurs. This is analogous to the IR-sensitive Bethe state-dependent logarithm in the Lamb Shift in hydrogen atoms in QED.

The physical IR divergence of the multi-gluon exchange $H$ diagrams in pQCD signals that QCD can only be consistent if color is confined - a remarkable fact. This can be contrasted with the fact that the Landau pole, which also produces an IR divergence, does not imply confinement since the pole is unphysical.

Other measured or calculated effective charges can be related to $\alpha_{V}$, or more generally, to any other effective charge by Commensurate Scale Relations (CSR) [50]. The $C S R$ are RS-independent. The relation between two schemes $A$ and $B$ is of the form:

$$
\frac{\alpha_{A}(Q)}{\pi}=\frac{\alpha_{B}\left(Q^{*}\right)}{\pi}+a\left(\frac{\alpha_{B}\left(Q^{* *}\right)}{\pi}\right)^{2}+b\left(\frac{\alpha_{B}\left(Q^{* * *}\right)}{\pi}\right)^{3}+\cdots
$$

The commensurate scale, $Q^{*}$, gives the mean virtuality of the exchanged gluon and thus the number of effective heavy quark flavors $n_{f}$. One has $Q^{*}=Q$ for $\alpha_{V}$, just like the scale $\mu$ in the QED coupling is set by the virtuality of the photon. At leading order (LO), $Q=1.18 Q^{*}$ for $\alpha_{g_{1}}, Q=1.36 Q^{*}$ for $\alpha_{\tau}$ and $Q=0.435 Q^{*}$ for $\alpha_{\overline{M S}}$. The first equality indicates that the $V$-scheme and $g_{1}$-scheme are similar, $\alpha_{g_{1}}(Q) \sim \alpha_{V}(Q)$, while $\alpha_{\overline{M S}}(Q)<\alpha_{V}(Q)$ since having $Q^{*}>Q$ is equivalent to shifting the Landau pole to a lower scale, see Fig. 3.4. Re-expressing the LO of Eq. (3.15) with $\mu=Q$ and $\mu=Q^{*}$ yields

$$
\alpha_{s}\left(Q^{2}\right)=\frac{\alpha_{s}\left(Q^{* 2}\right)}{1+\frac{\beta_{0}}{4 \pi} \ln \left(\frac{Q^{2}}{Q^{* 2}}\right)}=\alpha_{s}\left(Q^{* 2}\right) \sum_{n=0}^{\infty}\left[-\frac{\beta_{0}}{4 \pi} \ln \left(\frac{Q^{2}}{Q^{* 2}}\right)\right]^{n}
$$

which shows that the shift of scales amounts to re-organizing the perturbative expansion in $\alpha_{s}$ of an observable. The Commensurate Scale Relations have been shown to hold to any order of pQCD [51].

\subsubsection{The Brodsky, Lepage and Mackenzie procedure and its extensions}

The Brodsky, Lepage and Mackenzie (BLM) procedure [52] is a widely used method to optimize the perturbative series by setting the renormalization scales of the running coupling $\alpha_{s}^{n}\left(Q_{n}^{*}\right)$ at each order $n$ to absorb all $\beta$ terms. The coefficients of the pQCD series then matches the corresponding "conformal" series with $\beta=0$. The resulting predictions are then RS-invariant. 


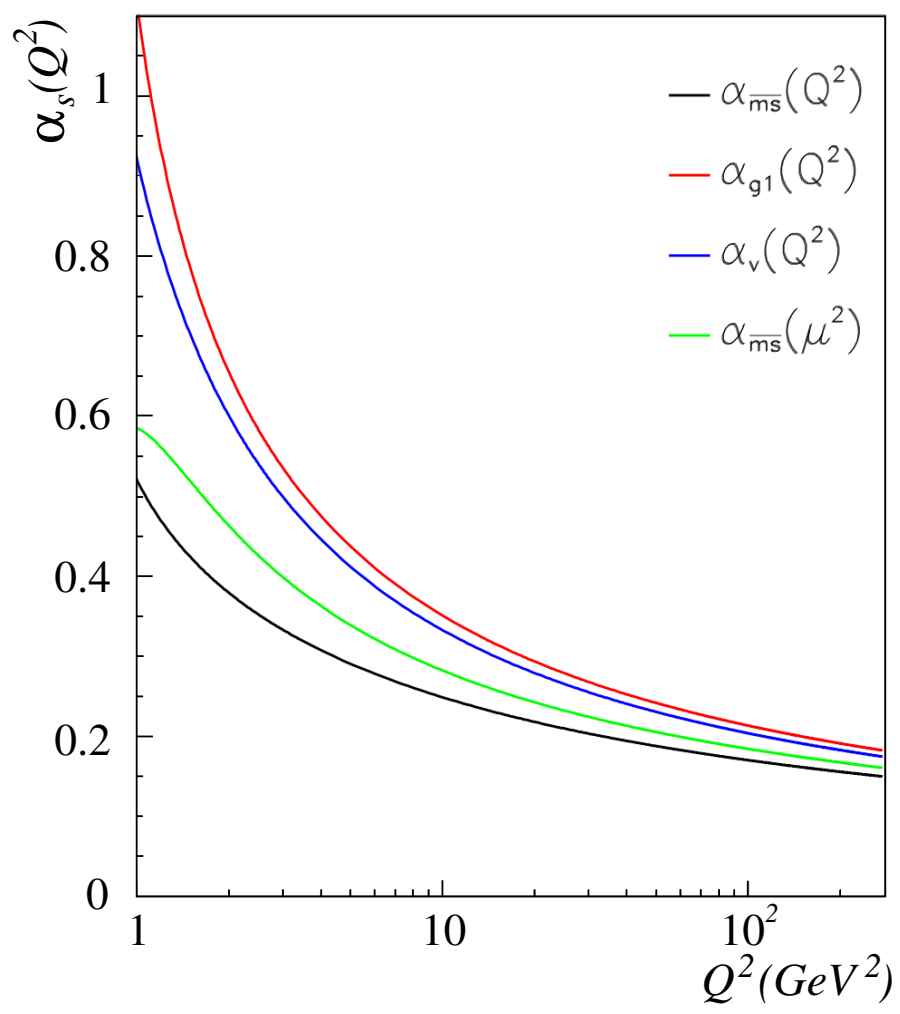

Figure 3.4: The strong coupling $\alpha_{s}\left(Q^{2}\right)$ expressed in different renormalization schemes (black: $\overline{M S}$-scheme, red: $g_{1}$ effective charge, blue $V$-scheme) and using a scale $\mu \neq Q$ (green). We chose $\mu=0.708 Q$ which, in the CSR context, is the LO scale shift transforming $\alpha_{\overline{M S}}$ into $\alpha_{R}$ $\left(\alpha_{R}\right.$ is the effective charge obtained from the ratio of the $e^{+}+e^{-} \rightarrow$ hadrons rate to that for $\left.e^{+}+e^{-} \rightarrow \mu^{+}+\mu^{-}\right)$. In this figure, $\alpha_{\overline{M S}}$ was computed with $n_{f}=3, \Lambda=0.34 \mathrm{GeV}$ and to order $\beta_{2}$.

The BLM procedure originates from the observation that in QED, the vacuum polarization contributions to the photon propagator are solely responsible for the running of $\alpha$. For example, in Möller scattering, the one-photon exchange amplitude depends on $\alpha(t)=\frac{\alpha(0)}{1-\Pi(t)}$ and $\alpha(u)=\frac{\alpha(0)}{1-\Pi(u)}$ which sums all vacuum polarization contributions to all orders.

For QCD, BLM showed that one can extend the QED scale-setting procedure to NLO in pQCD by identifying the $\beta_{0}$ and $\beta_{1}$ terms in the pQCD series from their unique dependence on $n_{f}$ and absorbing them by shifting the renormalization scale. As in QED, the resulting scales characterize the virtuality of the propagators in the amplitude and the effective number of flavors. The BLM procedure reduces to the Gell-Mann-Low scale setting in the Abelian limit $N_{C} \rightarrow 0$.

More explicitly, the NLO expression of an observable can be rewritten in a form 
making its $n_{f}$-dependence explicit:

$$
R=r_{0} \frac{\alpha_{s}^{n}(\mu)}{\pi}\left(1+\left[A+B n_{f}\right] \frac{\alpha_{s}(\mu)}{\pi}\right) .
$$

Using the RG Eqs. (3.15) and (3.35), Eq. (3.45) becomes

$$
R=r_{0} \frac{\alpha_{s}^{n}\left(\mu e^{3 B / n}\right)}{\pi}\left(1+\left[A+\frac{33}{2} B\right] \frac{\alpha_{s}\left(\mu e^{3 B / n}\right)}{\pi}\right)
$$

with the scale shifting $\mu \rightarrow \mu e^{3 B / n}$.

The BLM procedure was extended to next-to-next-to leading order (NNLO) by Brodsky and $\mathrm{Lu}[50]$. The renormalization scale $\mu^{2}\left(q_{3}^{2}, q_{2}^{2}, q_{3}^{2}\right)$ for the three-gluon vertex as a function of the Feynman virtualities of the three external gluons is given in [53] Extensions to higher orders are not as straightforward because of additional $n_{f}$-dependences due to processes unrelated to the running of $\alpha_{s}$ such as the UV finite corrections to the three and four-gluon vertices.

Several extensions of the BLM procedure have been proposed. An early extension of the BLM procedure was proposed by Neubert in Ref. [54], along with an explanation for the appearance of the $1 / Q^{2}$ power corrections at low momentum transfer. The sequential extended BLM procedure proposed by Kataev and Mikhailov [55] builds on Neubert's proposal. This extension has been recently developed further by Ma et al. [56]. As we discuss in the next section, a rigorous procedure for identifying the $\beta$ terms and setting the scales at any order in pQCD is given by the Principle of Maximum Conformality.

The BLM procedure and its extensions are physically motivated, minimizing the unphysical RS-dependence of the series. This is achieved by shifting the scale $\mu$ so that at all orders of Eq. (3.5), the RS-dependence of $\alpha_{s}$ cancels the RS-dependence of $r_{n}$. Other procedures have been developed in which the physical basis is the non-relativistic heavy quark phenomenology [57] or the effects of gluon radiation [58].

\subsubsection{Principle of maximum conformality}

The Principle of Maximum Conformality [59, 60] provides a rigorous generalization of the BLM procedure. In this method, one first generalizes the dimensional regularization by subtracting an extra constant $\delta$, in addition to the $\ln 4 \pi-\gamma_{E}$ subtraction which defines

the standard $\overline{M S}$ scheme. This defines the $R_{\delta}$ scheme. The coefficients of $\delta^{n}$ in the resulting pQCD series uniquely identify the $\beta$ terms and the pattern of their occurrence 
at every order. The $R_{\delta}$ procedure thus systematically identifies the nonconformal $\beta$ contributions to any perturbative QCD series.

All nonconformal (i.e., scale-dependent) terms in the perturbative series describing an observable are thus identified at each order and resummed in $\alpha_{s}^{n}$ by shifting its argument, thus allowing the automatic implementation of the BLM/PMC procedure at all orders. One thus obtains a perturbative series that is maximally conformal and thus scheme-independent. The BLM/PMC procedure restores the original purpose of introducing a running coupling and resolves the renormalon and RS-dependence ambiguities.

The elimination of the renormalization scale ambiguity greatly increases the precision, convergence, and reliability of pQCD predictions. For example, $P M C$ scale-setting has been applied to the pQCD prediction for $t \bar{t}$ pair production at the LHC, where subtle aspects of the renormalization scale of the three-gluon vertex and multi-gluon amplitudes, as well as large radiative corrections to heavy quarks at threshold, play a crucial role. The large discrepancy of pQCD predictions with the $t \bar{t}$ forward-backward asymmetry measured at the Tevatron is significantly reduced from $3 \sigma$ to approximately $1 \sigma$.

In general, amplitudes of the same order may have different renormalization scales. For example, the $t$-channel and $u$-channel photon-exchange amplitudes appearing in Möller scattering in QED at lowest order have scales $\mu^{2}=t$ and $\mu^{2}=u$, respectively, in the Gell-Mann Low scheme. In addition, new scales will appear at each higher order. The number of effective leptons $n_{\ell}$ in the QED $\beta$ function also changes. For example, the renormalization scales of the two-photon exchange amplitudes in Möller scattering have a smaller size than the scales of the Born amplitude since the two photons share the overall virtuality.

Distinctive renormalization scales have important phenomenological consequences for pQCD, especially in processes which are sensitive to the interference between contributing amplitudes such as the forward-backward heavy quark asymmetries. In each case, the correct scales will be set automatically by applying the PMC.

It should be emphasized that the conventional procedure of guessing a single renormalization scale and its range gives pQCD results for physical observables that depend on the renormalization scheme, contrary to the principles of the renormalization group. The conventional procedure is also clearly incorrect for QED. Varying the renormalization scale over a fixed range does not give a reliable method for estimating uncertainties in a pQCD expansion since the variation only exposes the nonconformal $\beta$-dependent terms. 


\subsection{Other optimization procedures}

We have discussed the BLM/PMC prescription and Commensurate Scale Relations in the previous sections. Other optimization procedures exist. We briefly discuss here the Fastest Apparent Convergence (FAC) principle, Principle of Minimal Sensitivity (PMS) and Optimized Perturbation Theory (OPT). The goal of these approaches is to devise an optimization procedure that sets the value of the first ambiguously defined (RSdependent) parameter of the $\beta$-series $\left(\beta_{2}\right)$. The 3-loop perturbative correction to a given observable (often unknown at the time when these ideas were developed) is minimized by the optimization. This leads to the removal of the Landau pole and generally makes the coupling observable dependent.

The FAC principle [4] is related to effective charges in the sense that it fixes the scale $\mu$ so that all perturbative coefficients beyond a given order are set to zero. However, the FAC procedure is not meant to be a scale-setting procedure.

The Principle of Minimal Sensitivity [61] assumes that, since observables should be RS-independent, their best approximations obtained via perturbation theory should be stable under small RS variations. In practice, this is realized by imposing the independence of $\alpha_{s}$ with respect to $\Lambda$ and the other RS-dependent parameters of a given perturbative series. However, as shown by Kramer and Lampe [62], the resulting PMS scales can be unphysical; for example, in the case of $e^{+} e^{-} \rightarrow q \bar{q} g$ the PMS scale grows without bound when the gluon virtuality becomes soft.

Optimized Perturbation Theory (OPT) is based on the PMS procedure [61]. The convergence of the perturbative expansion, Eq. (3.2), truncated to a given order $n_{t}$, is enhanced by requesting its independence from the choice of RS. The optimization is implemented by identifying the RS-dependent parameters in the $n_{t}$-truncated series (the $\beta_{n}$ for $2 \leq n \leq n_{t}$ and $\Lambda$ ), and requesting that the partial derivative of the perturbative expansion of the observable with respect to the RS-dependent parameters vanishes. Demanding RS-independence modifies the series coefficients $r_{n}\left(1 \leq n \leq n_{t}\right)$ and the coupling $\alpha_{s}$ to "optimized" values $\widetilde{r_{n}}$ and $\widetilde{\alpha_{s}}$. At first order the requirement of RSindependence imposes that Eq. (3.2) satisfies the Callan-Symanzik equation (3.4) with $\beta$ truncated at order $n_{t}$. This implies that the perturbative coefficients re-acquire a scale dependence: $r_{n}\left(Q^{2}, \mu^{2}, x_{f}\right)$. This approach is based on a convergence criterion rather than physical criteria as for effective charges or the BLM procedure and its extensions, or a simplicity criterion as for the $\overline{M S}$ scheme. In addition, the OPT redefines $\alpha_{s}$ since part of the scale dependence present in the usual definition is factored out of it and 
included back in the $r_{n}\left(Q^{2}, \mu^{2}, x\right)$ coefficients. In the context of the PMS/OPT, given the perturbative expansion of an observable and of $\alpha_{s}$ at order $n_{t}$, one can assess whether their combination is compatible with a small value of $\alpha_{s}$ at low momentum transfer. If so, the perturbative calculations can then be improved since the optimized coupling does not have a Landau pole and so, its growth at moderate momentum transfer is slower. This apparently allows one to extend the perturbative series to lower energies [61]. However, this procedure may hide situations where the physics of the subprocess requires consideration of nonperturbative dynamics. An example is $e^{+} e^{-} \rightarrow b \bar{b}$, where the scale of the final state gluon exchange is of order $v^{2} m_{b}^{2}$ and $v \rightarrow 0$ is the $b \bar{b}$ relative velocity.

It should be noted that the BLM/PMC scale-setting procedure automatically eliminates the dependence of the prediction on the choice of RS, so it automatically achieves the goals set by the PMS and OPT. The resulting renormalization scales are always physical, reflecting the virtuality of the amplitude and, as in QED, setting the number of active flavors $n_{f}$ appropriately at each order. The dependence on the choice of the initial scale $\mu_{0}$ is also minimized by the BLM/PMC procedure.

The $P M C$ method has now been applied to many collider processes including multiscale problems. The results are independent of the renormalization scheme and the procedure removes the problematic renormalon growth of the perturbative expansion, eliminates an unnecessary theoretical systematic error, and gives increased precision of the pQCD predictions. This includes pQCD predictions for LHC processes, such as Higgs and top quark production, thus greatly improving the sensitivity of LHC measurements to new physics.

\subsection{Determination of the strong coupling $\alpha_{s}\left(M_{Z}^{2}\right)$ or the QCD scale $\Lambda$}

The coupling $\alpha_{s}$ in a convenient scheme, such as the $\overline{M S}$ scheme, can be extracted from a number of different measurements involving hadronic reactions. Constraints can also be obtained from lattice gauge theory calculations. A comprehensive review of typical measures is given in Refs. [63, 64]. The various determinations can be compared with each other by either evolving them to a common scale, typically the $Z^{0}$ mass $M_{Z}$, or by giving the value of $\Lambda$ taking the appropriate number of effective flavors $n_{f}$. There has been important progresses toward highly accurate and precise determinations of $\alpha_{s}\left(M_{Z}^{2}\right)$, 


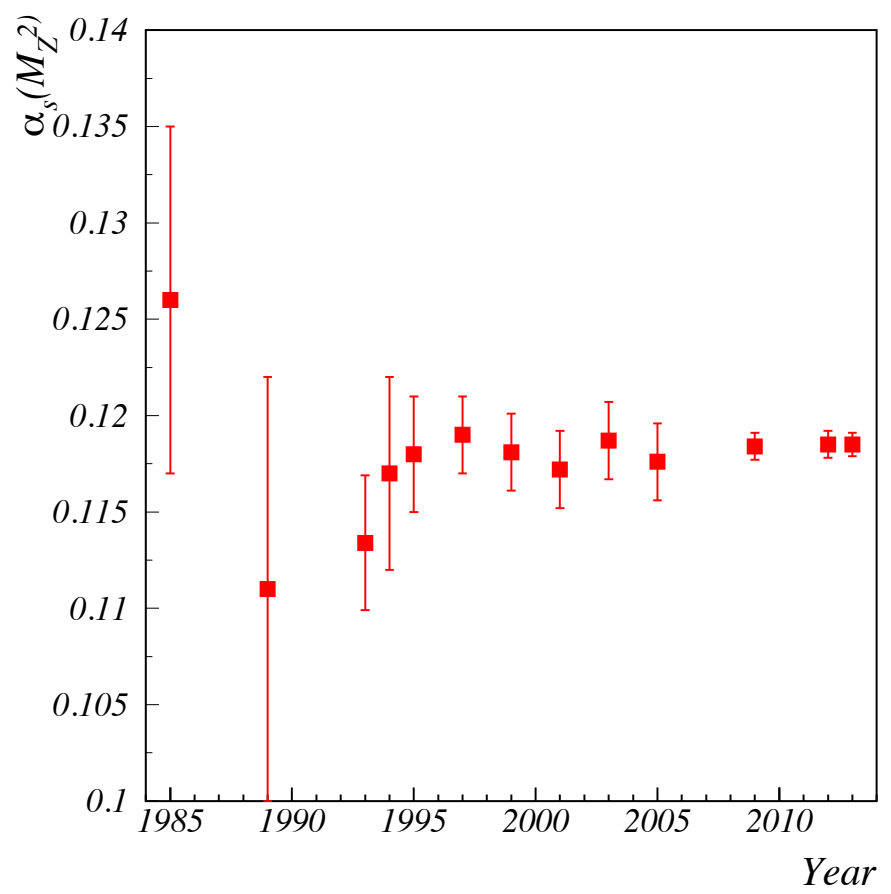

Figure 3.5: Evolution of the average world data for $\alpha_{s}\left(M_{Z}^{2}\right)$ (Particle Data Group average $[1])$.

as can be judged from Fig. 3.5 which summarizes our knowledge over the last 30 years as recorded by the Particle Data Group [1]. The present $Q^{2}$-range of the $\alpha_{s}$ determinations is approximately $0.05<Q^{2}<10^{3} \mathrm{GeV}^{2}$, although only data above $Q^{2}$ greater than a few $\mathrm{GeV}^{2}$ can be safely evolved with well-controlled perturbative equations. The large range provides an essential check on the pQCD prediction for the running of the coupling, Eq. (3.24), a fundamental check of the theory. In many cases, the precision could be improved further by fixing the renormalization scale using the $P M C$ procedure.

Several authors have compiled and compared the existing world data; see, for example, the recent Refs. $[1,3,65,66]$. The overall agreement between different determinations of $\alpha_{s}\left(M_{Z}^{2}\right)$ within about $4 \%$ provides an important consistency check of pQCD. There are nevertheless tensions between various measurements which are presently attributed to the underestimation of uncertainties, rather than the failure of QCD or effects from physics beyond the Standard Model. Typically, an Unbiased Estimate is obtained by fitting the set of $\alpha_{s}\left(M_{Z}^{2}\right)$ and scaling the individual uncertainties by an overall factor, until the $\chi^{2} / n d f$ of the fit reaches unity in order to account for underestimated uncertainties and for possible correlations between different determinations of $\alpha_{s}\left(M_{Z}^{2}\right)$. For the most precise determinations, theoretical uncertainties usually dominate. 
We summarize in this section the most effective methods for obtaining $\alpha_{s}\left(M_{Z}^{2}\right)$. In general, the conditions for a competitive extraction of $\alpha_{s}$ are a precisely measured quantity, as inclusive as possible, with high sensitivity to $\alpha_{s}$. In the case of hadron production in $e^{+} e^{-}$collisions, QCD enters as a correction to the electroweak process, so the corresponding observables are only moderately sensitive to $\alpha_{s}$. In contrast, hadronic decays of heavy mesons are directly proportional to $\alpha_{s}^{3}$. The perturbative expression of the quantity must also be accurately known to high enough order, typically NNLO. The measurement must be done either at large $Q^{2}$ to suppress unknown nonperturbative $1 / Q^{n}$ power corrections, or at low $Q^{2}$ but with small nonperturbative corrections. The evolution from low $Q^{2}$ to $M_{Z}^{2}$ then suppresses the overall uncertainties.

After describing the experimental status of $\alpha_{s}$, we will discuss several non-experimental methods for predicting $\alpha_{s}$. We then conclude by comparing some of the recent world data.

\subsubsection{Deep Inelastic Scattering}

The basic process underlying deep inelastic lepton-hadron scattering (DIS) is the elementary lepton-quark scattering process $\ell q \rightarrow \ell^{\prime} q^{\prime}$. The DIS (inclusive) data are sensitive to $\alpha_{s}$ through violations of Bjorken scaling induced at LO by gluon bremsstrahlung from the struck quark as well as the photon-gluon fusion and pair creation processes underlying the pQCD $Q^{2}$-evolution equations (Dokshitzer-Gribov-Lipatov-Altarelli-Parisi (DGLAP) equations [67]). At NLO, the quark-photon vertex and the quark self-energy corrections enter. DIS data arguably provide the most robust way to obtain $\alpha_{s}\left(M_{Z}^{2}\right)$ since the observables - the nucleon unpolarized and polarized structure functions $F_{2}\left(x_{f}, Q^{2}\right)$, $g_{1}\left(x_{f}, Q^{2}\right)$ and $F_{3}\left(x_{f}, Q^{2}\right)$ - are fully inclusive, and thus have no uncertainties from finalstate hadronic corrections.

The choice of the kinematic domain where pQCD analyses have been applied to DIS data is typically set at $Q^{2}>1$ or $2 \mathrm{GeV}^{2}$ in order to minimize higher-twist power-law corrections arising from multiparton processes. One also limits the invariant mass $W>$ 2 to $4 \mathrm{GeV}$ in order to exclude final-state high mass resonances, and one takes $x_{f}>0.01$ to avoid low- $x_{f}$ resummation problems. High- $x_{f}$ values are also excluded, since highertwist subprocesses can become significant in this domain [68]. The precision of the determinants of $\alpha_{s}\left(M_{Z}^{2}\right)$ from DIS measurements is at the percent level due to the high precision of the measurements of the unpolarized structure function $F_{2}\left(x_{f}, Q^{2}\right)$-now reaching $1 \%$ level over a wide kinematic range. The precision of the strong coupling $\alpha_{s}\left(M_{Z}^{2}\right)$ in this case is limited by uncertainties of the gluon density distribution. The 
theoretical understanding underlying the evolution of the polarized structure function $g_{1}\left(x_{f}, Q^{2}\right)$ is well developed, but the precision of the data needs further improvement.

Precise measurements of $\alpha_{s}$ could in principle be obtained from the $Q^{2}$-dependences of observables known to NNLO such as the Bjorken [36] or the Gross-Llewellyn Smith (GLS) sum rules [69]. The cleanest DIS observable for accessing $\alpha_{s}\left(M_{Z}^{2}\right)$ is the Bjorken integral, $\int_{0}^{1} d x_{f}\left(g_{1}^{p}\left(x_{f}, Q^{2}\right)-g_{1}^{n}\left(x_{f}, Q^{2}\right)\right)$. As such, the Bjorken integral $Q^{2}$-dependence is particularly simple and known up to $\mathrm{N}^{3} \mathrm{LO}$, see Eq. (3.40). Since it is non-singlet in isospin, there is no gluon distribution input, and thus an absolute and rigorous pQCD prediction exists (the Bjorken sum rule [36]). In practice, however, the input data lacks precision because one requires doubly polarized measurements on both the neutron and proton. Furthermore, the most precise measurements are at relatively low $Q^{2}, Q^{2} \leq 5$ $\mathrm{GeV}^{2}$, see [70]. Finally, there is an important uncertainty arising from the unmeasured low $-x_{f}$ part of the integral. These caveats more than counterbalance the advantages of the observable, and thus the resulting $\alpha_{s}\left(M_{Z}^{2}\right)$ is not quite yet competitive with the best extractions of $\alpha_{s}\left(M_{Z}^{2}\right)$. The fit of the $Q^{2}$-dependence of the Bjorken integral was used first in Ref. [71]. The latest measurements incorporate recent DIS data from Jefferson Lab's Hall B, yielding $\alpha_{s}\left(M_{Z}^{2}\right)=0.1123 \pm 0.0061$ [70]. Since relatively low $Q^{2}$ data points are included, the nonperturbative higher-twist correction $\mu_{4}^{p-n} / Q^{2}$ to the $Q^{2}$-dependence of the Bjorken integral has been accounted for with $\mu_{4}^{p-n}$ of the order of $-0.02 \mathrm{GeV}^{2}$. The $\mu_{6}^{p-n} / Q^{4}$ and higher order power corrections could be neglected.

The GLS sum rule [69], which relates the number of valence quarks in the nucleon to its $F_{3}\left(x_{f}, Q^{2}\right)$ structure function, offers the same advantages as the Bjorken sum rule. In the $\overline{M S} \mathrm{RS}$ it reads:

$$
\begin{aligned}
\int_{0}^{1} d x_{f} F_{3}\left(x_{f}, Q^{2}\right)=3\left[1-\frac{\alpha_{\overline{M S}}}{\pi}-\right. & 3.58\left(\frac{\alpha_{\overline{M S}}}{\pi}\right)^{2} \\
& \left.-18.98\left(\frac{\alpha_{\overline{M S}}}{\pi}\right)^{3}+\mathcal{O}\left(\alpha_{\overline{M S}}^{4}\right)\right]+\mathcal{O}\left(1 / Q^{2}\right),
\end{aligned}
$$

with the same perturbative correction as the Bjorken sum rule, except for a small contribution at order $\alpha_{s}^{3}$ from a light-by-light box-graph contribution. However, the experimental difficulties in measuring the GLS sum are even greater than for the Bjorken sum since it involves neutrino DIS measurements, and thus no precise determinations of $\alpha_{s}\left(M_{Z}^{2}\right)$ from the GLS sum rule exist at the moment. The available data for the GLS sum rule come from the CCFR measurement [72].

The $Q^{2}$-dependence of structure function moments can be used to determine $\alpha_{s}$, 
even without a sum rule to anchor its absolute magnitude. Bernstein polynomials can be inserted into the moments, to weight the integrand over the measured domain, thereby suppressing the uncertainties from the extrapolations to large and low $x_{f}$ [73].

As we have noted, the most precise values of $\alpha_{s}\left(M_{Z}^{2}\right)$ from DIS are at present obtained from structure functions or parton distribution functions global fits. Blumlein and collaborators [74] have combined the world data for structure functions. The analysis at NNLO, yields $\alpha_{s}\left(M_{Z}^{2}\right)=0.1133 \pm 0.0015$ (we have included a theoretical uncertainty not quoted by the authors and estimated from [75]) or $\alpha_{s}\left(M_{Z}^{2}\right)=0.1134 \pm 0.0020$, if only non-singlet structure functions are included. Another analysis from JimenezDelgado and Reya [76], which incorporates most of the world data for parton distributions, leads to $\alpha_{s}\left(M_{Z}^{2}\right)=0.1136 \pm 0.0014$. The NNPDF collaboration has extracted $\alpha_{s}\left(M_{Z}^{2}\right)=0.1191 \pm 0.0006$, with a small systematic uncertainty estimated using neural networks $[63,77]$.

Jet production in DIS provides another observable for determining $\alpha_{s}$. For example the production rate based on the subprocess $\gamma^{*} q \rightarrow q g$ is directly proportional to $\alpha_{s}$. The phenomena underlying the production of a gluon jet are the same as the ones responsible for the DGLAP $Q^{2}$-dependence of the DIS structure functions: bremsstrahlung and photon-gluon fusion. The appropriate kinematic domain is high $Q^{2}$ and large values of the mean transverse energy of the two highest energy jets. If one extracts $\alpha_{s}$ from the resulting scaling violations, the precision is limited by the correlation between $\alpha_{s}$ and the gluon distribution. An advantage of this technique is that it allows one to directly measure the $Q^{2}$-dependence of $\alpha_{s}$. A recent extraction from neutral-current DIS measurements of inclusive jet, dijet and trijet cross-sections at HERA (H1 collaboration) compared to the NLO expressions yields $\alpha_{s}\left(M_{Z}^{2}\right)=0.1160 \pm 0.0034$ [78].

\subsubsection{Observables from $e^{+} e^{-}$collisions}

Observables from $e^{+} e^{-}$collisions provide other inclusive processes to extract $\alpha_{s}\left(M_{Z}^{2}\right)$. At leading order, the $e^{+} e^{-}$pair annihilates into a virtual photon or a $Z^{0}$ which in turn decays into a quark-antiquark pair. At the $Z^{0}$-pole the observables are the $Z^{0}$ decay width $\Gamma_{Z}$, the ratio $R_{Z}$ of the $Z^{0}$ hadronic decay width normalized to the leptonic width, and the hadronic and leptonic cross-sections. The leptonic cross-section involves the $\Gamma_{Z}$ width and thus is sensitive to $\alpha_{s}$. Away from the pole, virtual photon production competes with $Z^{0}$ production. One can measure the ratio of the hadron production to lepton pair-production. In each case, the sensitivity to $\alpha_{s}$ comes from $\Gamma_{Z}$ and its predominant hadronic decays. The experimental set-ups are distinct, and thus yield 
results with largely independent and uncorrelated experimental systematic uncertainties. Ratios such as $R_{Z}$ and $R_{\tau}$ are experimentally robust and thus have smaller systematic uncertainties. The sensitivity to $\alpha_{s}$ originates at LO from bremsstrahlung on the quarkantiquark lines into which the $Z^{0}$ or photon have decayed. As it is the case for DIS, vertex and quark self-energy corrections enter at NLO. The pQCD corrections to the $e^{+} e^{-}$collision observables are usually known to $\mathrm{NNLO}$ or to $\mathrm{N}^{3} \mathrm{LO}$ for $R_{Z}$ and $R_{\tau}$ [79]. The uncertainties of the evolution equations are small, and thus the dominant uncertainty on the extraction of $\alpha_{s}$ is mostly experimental.

The $\mathrm{N}^{3} \mathrm{LO}$ expression for $R$ is:

$$
\begin{aligned}
& R\left(Q^{2}\right)=R_{E W}\left(1+\frac{\alpha_{s}\left(Q^{2}\right)}{\pi}+\left[1.9857-0.1153 n_{f}\right] \frac{\alpha_{s}^{2}\left(Q^{2}\right)}{\pi}\right. \\
&+\left[-6.6369-1.2011 n_{f}-0.0052 n_{f}^{2}-1.240 \eta\right] \frac{\alpha_{s}^{3}\left(Q^{2}\right)}{\pi} \\
&+\left[-156.61-18.775 n_{f}-0.7974 n_{f}^{2}+0.0215 n_{f}^{3}+\left(17.828-0.575 n_{f}\right) \eta\right] \frac{\alpha_{s}^{4}\left(Q^{2}\right)}{\pi} \\
&\left.+\mathcal{O}\left(\alpha_{s}^{5}\left(Q^{2}\right)\right)+\delta_{N P}\right),
\end{aligned}
$$

where $R_{E W}$ is the pure electroweak expectation, $\eta=\left(\Sigma e_{q}\right)^{2} /\left(3 \Sigma e_{q}^{2}\right)$ with $e_{q}$ the quark electric charges, and $\delta_{N P}$ includes the nonperturbative power corrections.

A useful final state from $Z^{0}$ decay is a $\tau^{+} \tau^{-}$pair. The $\tau$ can in turn undergo hadronic or leptonic decays, the ratio of which, $R_{\tau}$, allows the extraction of $\alpha_{s}$ at the low momentum scale $M_{\tau}^{2}=3.157 \mathrm{GeV}^{2}$ [80]. This leads to an accurate $\alpha_{s}\left(M_{Z}^{2}\right)$ determination, mostly because of the inclusiveness of $R_{\tau}$ and because the evolution from $M_{\tau}^{2}$ to $M_{Z}^{2}$ suppresses the absolute experimental uncertainties by approximately a factor $\alpha_{s}^{2}\left(M_{\tau}^{2}\right) / \alpha_{s}^{2}\left(M_{Z}^{2}\right) \simeq 7$. However, low momentum transfer measurements require the control of higher order pQCD corrections as well as nonperturbative power corrections. For $R_{\tau}$, the nonperturbative corrections are suppressed as $M_{\tau}^{6}$ and are estimated [81]. However, it is unclear that the perturbative corrections are determined properly for the most precise extractions, since different approaches to the perturbative series yield incompatible results, see e.g., Refs. [63] or [66]. One pQCD expansion is done in the fixed order perturbation theory (FOPT), while the other is done in the contour-improved perturbation theory (CIPT). The pQCD expansion of $R_{\tau}$ is derived from the vector and axial-vector current correlation functions. In the $\overline{M S}$ scheme the pQCD approximant 
of $R_{\tau}$ is:

$$
\begin{aligned}
R_{\tau}=N_{C}\left|V_{u d}\right|^{2} S_{E W}\left(M_{\tau}, M_{Z}\right)\left[1+A_{1}+1.63982 A_{2}\right. & +6.37101 A_{3} \\
& \left.+49.07570 A_{4}+\mathcal{O}\left(A_{5}\right)+\delta_{N P}\right],
\end{aligned}
$$

where $S_{E W}=1.01907 \pm 0.0003$ accounts for the electroweak radiative corrections, $\delta_{N P} \simeq$ -0.006 is the nonperturbative correction and $A_{n}$ are contour integrals depending, at LO, on $\alpha_{s}$ only:

$$
A_{n}=\frac{1}{2 \pi i} \oint_{|s|=M_{\tau}^{2}} \frac{d s}{s}\left(\frac{\alpha_{s}(s)}{\pi}\right)^{n}\left(1-2 \frac{s}{M_{\tau}^{2}}+2 \frac{s^{3}}{M_{\tau}^{6}}-2 \frac{s^{4}}{M_{\tau}^{8}}\right) .
$$

FOPT expands the $A_{n}$ in $\alpha_{s}, A_{n}=\alpha_{s}^{n}\left(M_{\tau}^{2}\right) / \pi+\mathcal{O}\left(\alpha_{s}^{n+1}\left(M_{\tau}^{2}\right) / \pi\right)$, while CIPT uses a numerical approach to keep the $A_{n}$ unexpanded; i.e., the higher orders in $\alpha_{s}$ are resummed compared to the FOPT perturbative series. The FOPT expansion yields:

$$
\begin{aligned}
R_{\tau}=N_{C}\left|V_{u d}\right|^{2} S_{E W}\left(M_{\tau}, M_{Z}\right)\left[1+\frac{\alpha_{s}\left(M_{\tau}^{2}\right)}{\pi}\right. & +5.202 \frac{\alpha_{s}^{2}\left(M_{\tau}^{2}\right)}{\pi}+26.37 \frac{\alpha_{s}^{3}\left(M_{\tau}^{2}\right)}{\pi} \\
& \left.+127.1 \frac{\alpha_{s}^{4}\left(M_{\tau}^{2}\right)}{\pi}+\mathcal{O}\left(A_{5}\right)+\delta_{N P}\right]
\end{aligned}
$$

The question of which expansion is preferable has not yet been settled. It has been argued that the FOPT uncertainties have been underestimated, and if they are properly calculated, the two techniques will agree -see the contribution of S. Menke to Ref. [63]. The latest analysis of $\tau$-decay data, using RG-improved FOPT expansion, yields $\alpha_{s}\left(M_{\tau}^{2}\right)=0.3189_{-0.0151}^{+0.0173}$, corresponding to $\alpha_{s}\left(M_{Z}^{2}\right)=0.1184_{-0.0018}^{+0.0021}$ [82]. The experimental data are from the ALEPH [83] and OPAL [84] experiments at LEP (CERN) .

Observables based on jet shapes can also be used to access $\alpha_{s}\left(M_{Z}^{2}\right)$. These observables are less inclusive but are sensitive to $\alpha_{s}$ at leading order. Event shapes measure the departure of the momentum flow in an event from that of the 2-body $q \bar{q}$ configuration. Extractions are carried out at NNLO. Uncertainties from nonperturbative hadronization processes dominate the total uncertainty. The shape observable that has provided the most precise determination of the coupling is the "thrust", $T$. It measures the alignment of the produced particles with respect to the thrust axis, defined as the axis on which the projected momenta of the produced particles is maximal. Thrust varies between $0.5<T<1$. The low values characterize the 3 -jet region where pQCD is applicable, and high values characterize the 2-jet region where nonperturbative ef- 
fects due to soft/collinear gluons are important. Recently, Gehrmann, Luisoni and Monni [85] used thrust data from the TASSO experiment at PETRA (DESY) [86] and the ALEPH [87] and L3 [88] experiments at LEP to obtain $\alpha_{R g a p}\left(M_{Z}^{2}\right)=0.1131_{-0.0022}^{+0.0028}$ and $\alpha_{\text {Rgap }}(2 \mathrm{GeV})=0.538_{-0.047}^{+0.102}$ (both in the Rgap-scheme). Another recent thrust result, from Abbate et al. [89], includes additional experimental data from JADE (PETRA) [90], OPAL [91], DELPHI [92] (LEP) and AMY [93] (at TRISTAN). It yields $\alpha_{s}\left(M_{Z}^{2}\right)=0.1135 \pm 0.0025$ ( $\overline{M S}$ scheme), in good agreement with an earlier more precise extraction by the same group, $\alpha_{s}\left(M_{Z}^{2}\right)=0.1135 \pm 0.0010$. Another very recent thrust result [94] (still unpublished) yields $\alpha_{R g a p}\left(M_{Z}^{2}\right)=0.1128 \pm 0.0012$, using ALEPH, DELPHI, JADE, OPAL and SLD (SLAC) [95] data. The same group also provides another determination using a different event-shape observable, the C-parameter distribution: $\alpha_{\text {Rgap }}\left(M_{Z}^{2}\right)=0.1123 \pm 0.0015$ (both results are in the Rgap scheme).

Another recent determination is from the analysis of the $Q^{2}$-evolution of the average gluon and quark jet multiplicities using a recently improved formalism. This analysis yields $\alpha_{s}\left(M_{Z}^{2}\right)=0.1199 \pm 0.0026$ [96]. A global electroweak fit of weak decay data by

Erler and Freitas [1], yields $\alpha_{s}\left(M_{Z}^{2}\right)=0.1192 \pm 0.0027$ with, noticeably, a negligible theoretical contribution to the uncertainty.

\subsubsection{Observables from $p p$ collisions}

Most of the determinations of $\alpha_{s}$ from hadronic collisions have been limited to NLO. However, the CMS collaboration at LHC has recently determined $\alpha_{s}$ from the inclusive cross section for top-quark pair $t \bar{t}$ production based on a NNLO analysis that is constrained by PDF inputs. This analysis yields $\alpha_{s}\left(M_{Z}^{2}\right)=0.1185_{-0.0042}^{+0.0063}$ [97].

Another important result of the high energy hadron colliders, the LHC and the Tevatron, is the experimental verification of the running of $\alpha_{s}\left(Q^{2}\right)$ at very large momentum transfers of order $1 \mathrm{TeV}$.

Inclusive jet production from $p \bar{p}$ collisions is proportional to $\alpha_{s}^{2}\left(Q^{2}\right)$ at LO, and it provides constraints on the global PDF fits of DIS and hard scattering data. The MSTW combined fit of DIS and jet data yields $\alpha_{s}\left(M_{Z}^{2}\right)=0.1180 \pm 0.0014$ at NLO [98].

\subsubsection{Lattice QCD}

Lattice QCD can provide accurate determinations of $\alpha_{s}\left(M_{Z}^{2}\right)$ at the $1 \%$ level, although it is not certain that all uncertainties are understood, see e.g., Refs. [3] or [63]. Consequently, attention has focused recently on a better understanding and control of 
the lattice systematic uncertainties. The systematic uncertainties quoted in the older calculations (10 years ago or earlier) are most likely underestimated and not well controlled. Recent unquenched calculations explicitly include quark loops up to $2+1$ or $2+1+1$ quark flavors. This is adequate for the momentum transfer range at which the calculations are performed $(2+1$ means that one of the sea quark masses is set to the strange quark mass, while the two others are taken as small as practically possible. For $2+1+1$, the charm quark mass is added).

A recent exhaustive review of the lattice results extracting $\alpha_{s}$ in the pQCD domain can be found in [99]. It also provides a compilation of the lattice results, yielding an average value for the coupling $\alpha_{s}\left(M_{Z}^{2}\right)=0.1184 \pm 0.0012$. In this Section, we first outline the method, its benefits and its limitations. We will then report on the most recent and most accurate determinations of $\alpha_{s}$ in the UV domain.

Lattice calculation technique Lattice calculations use the path integral formalism [100]. Path integrals provide the probability of evolving from an initial state $\left|x_{i}\right\rangle$ to a final state $\left|x_{f}\right\rangle$, summing over all possible space-time trajectories. The integral is weighted by a factor depending on the system's action, $S$. For example for a one-dimensional system the propagator is given by:

$$
\left\langle x_{f}\left|e^{-i H t}\right| x_{i}\right\rangle=\int D x(t) e^{-i S[x(t)] / \hbar}
$$

where $\int D x$ symbolizes the integration over all paths for which $x\left(t_{f}\right)=x_{f}$ and $x\left(t_{i}\right)=x_{i}$. In Eq. (3.52), we exhibit the dependence on $\hbar$ explicitly in order to underline the link between path integrals (quantum description) and the principles of Fermat/Maupertuis (the least action principle), which specify that the classical path $(\hbar \rightarrow 0)$ must yield the smallest value of $S$. The fact that $\hbar \neq 0$ allows excursions outside the classical path and is responsible for the quantum effects.

Path integrals are rarely solved analytically. They are also difficult to determine numerically since, for a 4-dimensional space, one requires an $n$-dimensional integration, with $n=4 \times$ (possible number of paths). Since the number of possible paths is infinite, one must restrict the number of paths to a representative sample. This integration is then carried on a finite sample. The most efficient technique for such numerical integrations is to use the Monte Carlo method. It is also more efficient to work in Euclidean rather than in Minkowski space. After a Wick rotation $i t \rightarrow t$ [101], the weighting factor becomes $e^{-S_{E}}$ which is easier to process than the oscillating function $e^{-i S}$. Here, $S_{E}$ denotes the 
Euclidean action. Using these methods, one can simply calculate correlation functions such as

$$
\left\langle A_{1} \ldots A_{n}\right\rangle=\frac{\int D x A_{1} \ldots A_{n} e^{-S_{E}}}{\int D x e^{-S_{E}}},
$$

where $A_{i}$ is the gauge field at position $x_{i}$. In particular, the two-point correlation function at $\left\langle x_{1} x_{2}\right\rangle$ represents the propagator of a boson. As soon as interacting fields are involved, no methods are known to directly solve Eq. (3.53) analytically. However, if the strength of the interaction is sufficiently weak, one can analytically evaluate the Gaussian integrals by expanding the exponential involving the interaction term (e.g. pQCD or QED). However, if the coupling is large, the integrals must be treated numerically. The numerical technique used is as follows: space is discretized (approximated by a lattice) and the paths linking the different sites (nodes of discretized space-time) are generated. The statistical precision depends on the square root of the number of generated paths. A path is generated according to the probability $e^{-S_{E}}$, where $S_{E}$ is calculated for that particular path. A correlation function can then be calculated by summing the integrand over all paths. Since paths are generated with the probability $e^{-S_{E}}$, this simple sum is equal to the weighted sum $\sum_{\text {path }} x_{1} \ldots x_{n} e^{-S_{E}} \simeq \int D x x_{1} \ldots x_{n} e^{-S_{E}}$.

The Monte Carlo technique can be used to generate paths with the appropriate weight [102]. The procedure begins with a given path of action $S_{1}$. The path is randomly changed to a new path of action $S_{2}$ (several intermediate paths, which are not retained, are generated before producing the $S_{2}$ path in order to avoid correlations between the $S_{1}$ and $S_{2}$ paths). If $S_{2}<S_{1}$ the $S_{2}$ path is retained in the sample. Otherwise, it is retained or rejected with probability $S_{2}-S_{1}$.

In order to ensure gauge invariance, a link involving the gauge field $A$ between the lattice sites must be introduced [103]. A link variable $U_{\vec{\mu}}=\exp \left(-i \int_{x}^{x+a \vec{\mu}} d y g A\right)$ is constructed, where $x$ is a lattice site, $a$ is the lattice spacing, $\vec{\mu}$ is an elementary vector of the Euclidean space, and $g$ the bare coupling. The link variable $U_{\vec{\mu}}$ is explicitly gauge-invariant. The action is then constructed using these gauge-invariant variables. The Wilson loop $U_{1} \ldots U_{n}$ thus appears [103], where the closed path is given by the links $U_{i}$. It is straightforward to show that the action can be expressed as a sum of Wilson loops. In the continuum limit $a \rightarrow 0$, the simplest loop (a square of side $a$ ) dominates. However, since $a \neq 0$ in the numerical simulations, larger loops must be introduced as corrections to the discretized expression of $S_{E}$.

We have discussed only $A$ (i.e. gluons) so far. The introduction of non-static quarks on the lattice is complicated because of their fermionic nature. Including a fermion 
field leads to the notorious fermion doubling problem, which multiplies the number of fermionic degrees of freedom, and introduces spurious particles. There are several possible methods which avoid this problem. One method is to break chiral symmetry (the Ginsparg-Wilson approach [104]). Another method, called "staggered fermions" introduces non-local operators which respect chiral symmetry [105]. Other methods also exist. Each of these methods significantly slow down the lattice computations. Once fermions are included, the action becomes $S_{E}=S_{A}-\ln (\operatorname{Det}(K))$ where $S_{A}$ comes from the gluon field and $K$ is similar to the Dirac equation operator. Most early Lattice calculations, and some recent ones, simplify the calculations by taking $\operatorname{Det}(K)=1$ (the quenched approximation). This amounts to neglecting the dynamics of fermions which eliminates the effects of pair creation from the QCD instant-time vacuum.

The lattice technique, although very powerful, has its own limitations:

Critical slowing down This phenomenon limits the statistical precision. The problem, which is not specific to lattice calculations, arises from the fact that, to keep discretization errors under control, the lattice spacing a must be much smaller than the characteristic sizes of the studied phenomena. The relevant physical measure is the correlation length $L_{c}$ defined by $\left\langle x_{1} x_{2}\right\rangle \sim e^{-x / L_{c}}$. In general $L_{c}$ is very small, except near a critical point. Therefore, calculations need to be performed near such point. However, when $L_{c}$ is large, many intermediate paths must be generated to obtain a path decorrelated from the initial path. In the case of QCD, the statistical precision varies as $\left(\frac{L_{R}}{a}\right)^{4}\left(\frac{1}{a} \frac{1}{m_{\pi}^{2} a}\right)$ where $L_{R}$ is the lattice size [106]. The first factor stems from the number of sites and the second comes from the critical slowing down (note the presence of the pion mass squared).

Extrapolation to the physical mass of the pion The lattice parameters can be chosen such that the mass of the pion is greater than its physical value, which minimizes the critical slowing down. This competes with the necessity to have calculations with the pion mass as close as possible from the physical mass. The lattice results are extrapolated to the physical pion mass with guidance from Chiral Perturbation Theory [107]. Nevertheless, an uncertainty remains associated with this extrapolation.

Local operators Local operators are well suited for lattice calculations because selecting a given path involves calculating the associated difference of actions $S_{2}-S_{1}$. For a local action, this amounts to computing $S_{2}-S_{1}$ only on one site and its neighbors 
(since $S$ involves derivatives). In four dimensions this represents 9 operations; in contrast if the action is not local, the calculation needs to be carried at each site of the lattice. This makes direct lattice calculations of non-local operators impractical. For example, structure functions are non-local, and thus they must be reconstructed on the lattice through their moments.

High momentum cut off Momenta involved in lattice calculations are automatically limited to $p \lesssim 1 / a$. This is not a problem in practice if one can match to available pQCD calculations. The domain where pQCD and lattice calculations are both valid allows one to establish the renormalization procedure for the lattice calculations.

Finite lattice size The lattice spacing $a$ must be chosen to be sufficiently small in order to reach the pQCD domain while keeping the number of sites to a practical value for computation. This limits the total lattice size. On the other hand, the lattice size must be taken large enough to encompass the physical system and to minimize boundary effects.

Severals approaches have been used to obtain $\alpha_{s}\left(M_{Z}^{2}\right)$ using lattice gauge theory. A first approach is to consider a short-distance quantity whose pQCD prediction is known to high order, and compute it on the lattice nonperturbatively. The result is compared to the pQCD prediction in which the value of $\alpha_{s}$ is adjusted so that there is a good match. The dominant uncertainty is usually from the perturbative series truncation. Lattice finite size effects are suppressed when short-distance quantities are computed. Space discretization errors are minimized by the use of improved actions, such as tadpole improved actions [106]. A second lattice approach consists of direct calculations; e.g., the computation of a QCD vertex.

The coupling from short-distance quantities A natural quantity to study on the lattice which determines $\alpha_{s}$ is the static Q- $\overline{\mathrm{Q}}$ potential at short distances [108]. This quantity will be discussed in more detail in Section 4.3. A recent review of the determination of $\alpha_{s}\left(M_{Z}^{2}\right)$ using this method is given in [49].

The energy between a static quark and a static anti-quark separated by a distance $r$ is calculated on the lattice. Unquenched results at short distances are then compared to the pQCD one-gluon static potential $V(r)=-4 \alpha_{R}(r) / 3 r$ allowing for an additional nonperturbative linear contribution $\sigma r$. The coupling in coordinate space $\alpha_{R}(r)$ is then 
Fourier transformed to $\alpha_{V}\left(Q^{2}\right)$.

At short distance, the 2-loop expression is [108]:

$$
\alpha_{R}(r)=\frac{6 \pi}{\beta_{0} \ln \left(\frac{1}{r^{2} \Lambda_{R}^{2}}\right)+\frac{4 \pi \beta_{1}}{\beta_{0}} \ln \left(\ln \left(\frac{1}{r^{2} \Lambda_{R}^{2}}\right)\right)} .
$$

The QCD parameter $\Lambda_{R}$ in the $R$-scheme is given in Table 3.1 for $n_{f}=3$. In the pure gauge case, $n_{f}=0$, it is $\Lambda_{R}^{0}=0.70 \mathrm{GeV}$. The most recent calculation yields a coupling $\alpha_{s}=0.335_{-0.010}^{+0.012}$ at $Q^{2}=1.5 \mathrm{GeV}^{2}$ for $n_{f}=2+1$, which is evolved to $\alpha_{s}\left(M_{Z}^{2}\right)=0.1166_{-0.0008}^{+0.0012}$ for $n_{f}=5$ [109]. The uncertainties shrink by an order of magnitude when evolving from $Q^{2}=1.5 \mathrm{GeV}^{2}$ to $M_{Z}^{2}$. For the quoted results, the light quark masses are set close to their physical values ( $5 \mathrm{MeV}$ for $u$ and $d$ ) or at their physical value (for $s$ ). Results are stable under variation of the value of the lattice spacing $a$, indicating a negligible discretization error. Another recent calculation, from the ETMC collaboration, was performed for $n_{f}=2$ and yields $\Lambda_{\overline{M S}}^{(2)}=0.332 \pm 0.0021$ [110], in good agreement with the world average.

Another lattice approach is to evaluate the temporal $\mathrm{n}^{\text {th }}$-moments $G_{n}$ of currentcurrent correlators $\left\langle 0\left|j_{5}(x, t) j_{5}(0,0)\right| 0\right\rangle$ for the heavy quark pseudoscalar current $j_{5}=$ $\bar{\psi} \gamma_{5} \psi$ [111]. As for the previous method, the currents are calculated on the lattice and compared to their pQCD expressions. The lattice results are fit using the pQCD functional form with several fit parameters, including $\alpha_{\overline{M S}}$. The pQCD expression is known to NNLO. For example for $n=4, G_{4}=G_{4}^{L O}\left[1+0.7427 \alpha_{\overline{M S}}(\mu)+0.0088 \alpha_{\overline{M S}}^{2}(\mu)-\right.$ $\left.0.0296 \alpha_{\overline{M S}}^{3}(\mu)\right]\left[1+\mathcal{O}\left(\Lambda^{4} / m^{4}\right)\right]$, where $m$ is the heavy quark mass. Higher pQCD orders are not known and are treated as free fit parameters up to $N^{14} L O$. This constrains the uncertainty on the truncation of the pQCD series. The nonperturbative terms are assumed to be proportional to gluon and meson condensates which are suppressed as $(\Lambda / m)^{4}$. Two results were obtained by the HPQCD collaboration. In the first case, the light quarks $u, d$ and $s$ were treated nonperturbatively, taking the heavy quarks as perturbative effects. This $n_{f}=2+1$ calculation yields $\alpha_{s}\left(25 \mathrm{GeV}^{2}\right)=0.2034 \pm 0.0021$. Evolved to the $Z^{0}$ mass and corrected to $n_{f}=5$, it gives $\alpha_{s}\left(M_{Z}^{2}\right)=0.1183 \pm 0.0007$. A second calculation was done with the $c$ quark also treated nonperturbatively. This analysis had improved statistics, as well as improved determinations of the gluon action and the lattice spacing. The $n_{f}=2+1+1$ calculation yields $\alpha_{s}\left(25 \mathrm{GeV}^{2}\right)=0.2128 \pm 0.0025$, which leads to $\alpha_{s}\left(M_{Z}^{2}\right)=0.11822 \pm 0.00074\left(n_{f}=5\right)$, confirming the earlier result, including the assumption that heavy quarks can be treated perturbatively. Calculations are done for several lattice spacings $a$, varying between $0.06 \leq a \leq 0.12 \mathrm{fm}$, and extrapo- 
lated to the continuum case. The value of $a$ is measured by calculating the dimensionless Wilson flow parameter $w_{0} / a$ and comparing it to its dimensionful value $w_{0}$ known from an earlier simulation. The light quark masses are chosen relatively close to their physical values and corrections for the finite lattice size effects are included. The JLQCD collaboration also used current-current correlators, but computed the vacuum polarization function $\Pi\left(Q^{2}\right)[112]$ rather than $G_{n}$ moments. The vector and axial-vector currents are both used. The $\mathrm{N}^{3} \mathrm{LO}$ pQCD expression is complemented by nonperturbative contributions up to $1 / Q^{4}$. The calculation yields $\alpha_{s}\left(M_{Z}^{2}\right)=0.1181 \pm 0.0013$. The chosen $u$ and $d$ quark masses range between 20 and $80 \mathrm{MeV}$. The $s$ quark mass ranges between 95 and $125 \mathrm{MeV}$. Discretization effects are estimated using lattice perturbation theory. The effects of the finite lattice size are found to be small.

The coupling $\alpha_{s}$ has also been extracted from the vacuum expectation values of Wilson loops $W_{m n}$-see Ref. [113] for a recent determination. The indices $m$ and $n$ characterize the loop which forms a rectangle of size $m a \times n a$. The loop definition is $W_{m n} \equiv\left\langle 0\left|\operatorname{Re} \operatorname{Tr} \mathrm{P} e^{-i g \oint_{n m} A d x}\right| 0\right\rangle / 3$, with $g$ defined as $\overline{\alpha_{s}}=g^{2} / 4 \pi$ and $\mathrm{P}$ is the path ordering operator. The corresponding pQCD expression for a flat $2 a \times 2 a$ loop is: $W_{22}^{p Q C D}=\exp \left[-9.20 \alpha_{V}(2.582 / a)+6.37 \alpha_{V}^{2}(2.582 / a)-17.11 \alpha_{V}^{3}(2.582 / a)+\ldots\right]$. In [113] calculations are done for various loop sizes and flat and non-flat loops, for a total of 22 different loops. The value of $\alpha_{V}$ is adjusted so that the NNLO pQCD expectation matches the lattice result. The averaging of the 22 different determinations yields $\alpha_{V}\left(56.25 \mathrm{GeV}^{2}\right)=0.2120 \pm 0.0028$. The conversion from the $V$-scheme to $\overline{M S}$ and evolution to the $Z^{0}$ mass lead to $\alpha_{s}\left(M_{Z}^{2}\right)=0.1184 \pm 0.0008$. The physical parameters of the simulation (the bare coupling constant and bare quark masses) are tuned so that the calculation reproduces the known experimental values of the $\mathrm{Y}^{\prime} \mathrm{Y}^{\prime}$ meson mass difference, of $m_{\pi}$, of $2 m_{K}^{2}-m_{\pi}^{2}$, of $m_{\eta_{c}}$ and of $m_{\Upsilon}$. Several values of lattice spacing are used to extrapolate to the continuum case. In addition, the calculations are done for several values of the light quark masses in order to reliably extrapolate to the physical case. The heavy quarks ( $c$ and $b$ ) are treated perturbatively. Finite lattice size effects are accounted for, as well as the truncation error on the pQCD series. Earlier high precision calculations using Wilson loops by Maltman et al. [114] and by the SESAM collaboration [115] yield $\alpha_{s}\left(M_{Z}^{2}\right)=0.1192 \pm 0.0011$ and $\alpha_{s}\left(M_{Z}^{2}\right)=0.1118 \pm 0.0017$, respectively.

Vertex calculations of $\alpha_{s}$ As will be discussed in more detail in Sections 4.4 and 4.5 , the running QCD coupling $\alpha_{s}$ can be computed from the ghost-gluon vertex, the 
quark-gluon vertex or the multi-gluon vertices. Vertices are not observable and their calculations are gauge-dependent. Most of the computations are done in the Landau gauge and in a MOM RS. A recent calculation by the ETM collaboration using the ghost-gluon vertex, see Eq. (4.25), yields $\alpha_{s}\left(M_{Z}^{2}\right)=0.1196 \pm 0.0014$ [116].

Another approach is to use the Schrödinger functional [117]. There are no high precision $\left(\Delta \alpha_{s} \lesssim 0.002\right)$ results or calculations with $n_{f} \geq 3$ yet, although some should become available soon [118].

\subsubsection{Heavy quarkonia}

Hadronic inclusive decay rates of heavy quarkonium systems are very sensitive to the value of $\alpha_{s}$ : for example, the LO hadronic decay rates of the $J^{P C}=1^{--}$bound states are proportional to $\alpha_{s}^{3}$ if the Zweig rule is operative. The dependence on the notwell-known quarkonium wave function is eliminated by considering the ratio of hadronic to leptonic decays. However, pQCD quarkonium decay rate expressions are known only to NLO and thus, the extractions have significant theoretical uncertainties. There is no recent extraction of $\alpha_{s}$ using this method. The latest one, from 2007, is $\alpha_{s}\left(M_{\Upsilon(1 S)}^{2}\right)=$

$0.184_{-0.014}^{+0.015}$, which is evolved to $\alpha_{s}\left(M_{Z}^{2}\right)=0.119_{-0.005}^{+0.006}$ [119]. It is extracted by comparing the measured $\Gamma(\Upsilon(1 S) \rightarrow \gamma X) / \Gamma(\Upsilon(1 S) \rightarrow X)$ decay ratio to non-relativistic QCD calculations (here, $X$ denotes final hadron states). The most precise rate measurement is provided by CLEO [120].

\subsubsection{Holographic QCD}

Light-Front Holographic QCD [121, 122] originates from the direct connection of QCD quantized on Dirac's light-front dynamics in our physical 3+1-dimensional spacetime [123], to Einstein's gravity in a 5-dimensional Anti-de Sitter. (AdS) space-time (An AdS space is the maximal symmetric space with constant negative curvature). The connection is based on the AdS/CFT correspondence [9], where CFT stands for conformal field theory, that is a theory without explicit scale dependence. The correspondence implies that a weakly interacting, gravity-like, theory in d+1-dimensional AdS space can be mapped on the (d-dimensional) AdS space boundary to a strongly interacting conformal field theory in d-dimensions, thus also the name gauge/gravity correspondence.

The holographic mapping to light-front physics gives a relation between the fifth dimension holographic variable of AdS space and the invariant impact light-front variable in physical space-time $[121,122]$. Light-front holography provides a precise relation 
between the boost-invariant light-front wavefunctions describing the internal structure of hadrons in physical space-time and the bound-state amplitudes in AdS space. This connection also implies that the light-front confining potential corresponds to an infrared distortion of AdS the space, which breaks the conformal invariance. The resulting valence Fock-state wavefunctions of the light-front QCD Hamiltonian satisfy a relativistic equation of motion with an effective confining potential which incorporates the contribution from higher Fock-states. Holographic QCD gives a very good description of hadrons of arbitrary spin and incorporates many of their observed spectroscopic and dynamical features [124]. The AdS/CFT correspondence offers new tools to analytically describe the strong interaction at low- $Q^{2}$. This technique can then be used to compute $\Lambda$ and in turn $\alpha_{s}\left(M_{Z}\right)$. The light-front holographic approach to hadronic physics and its recent connection with superconformal quantum mechanics is described in more detail in Sec. 4.2. We will also show in Sec. 4.2 how the running of the strong coupling in the infrared can be obtained from AdS gravity.

Following Sec.4.2, one can show how the mass scale underlying confinement and hadron masses determines the scale controlling the evolution of the perturbative QCD coupling [125]. The relation between scales is obtained by matching the nonperturbative dynamics, as described by an effective light-front theory embedded in AdS space, to the perturbative QCD regime computed to four-loop order. While the AdS/QCD description is valid only in the nonperturbative QCD regime, one can actually match it to the perturbative regime thanks to the existence of an overlap between both regimes, called the parton-hadron duality [126, 127]. The Holographic QCD predictions for $\alpha_{s}$ and its $\beta$-function are equated to their pQCD counterparts at a transition scale, which is in turn determined by the matching procedure. One thus derives a running QCD coupling $\alpha_{s}\left(Q^{2}\right)$, defined at all momenta, which is consistent with the measured effective charge defined from the Bjorken sum rule and with the measured perturbative scale $\Lambda_{\overline{M S}}$. At order $\beta_{0}$ and in the $\overline{M S}$ scheme, the relation is:

$$
\Lambda_{\overline{M S}}^{(L O)}=M_{\rho} e^{-a} / \sqrt{a}
$$

with $M_{\rho}$ the $\rho$ meson mass and $a=4\left(\sqrt{\ln (2)^{2}+1+\beta_{0} / 4}-\ln (2)\right) / \beta_{0} \simeq 0.55$. The result at $\beta_{3}$ and $n_{f}=3$ is $\Lambda_{\overline{M S}}=0.341 \pm 0.032 \mathrm{GeV}$ in good agreement with the combined world data, $\Lambda_{\overline{M S}}^{(3)}=0.340 \pm 0.008 \mathrm{GeV}$ [1]. The results are illustrated in Fig. 4.3 in Sec. 4.2, where the matching procedure and the derivation of $\alpha_{s}$ using light-front holographic methods is discussed in more detail. 
Eq. (3.55) can be alternatively expressed using the nucleon mass $M_{N}$ :

$$
\Lambda_{\overline{M S}}^{(L O)}=M_{N} e^{-a} / \sqrt{2 a}
$$

More generally one can use as input the mass of any meson or baryon composed of light quarks, since their masses are related to each other within the holographic QCD framework or, conversely, meson and baryon masses can be computed using only $\Lambda$ as input [125]. Such a relation between the perturbative scale $\Lambda$ and the hadron masses allows one to express the QCD fundamental mass scale as a function of a schemeindependent quantity rather than the scheme-dependent, and thus unphysical parameter, $\Lambda$. Since QCD has no knowledge of conventional units of mass such as GeV; only ratios are predicted. Consequently any calculation necessarily can only yield ratios such $\Lambda_{\overline{M S}} / M$, with $M$ any hadron mass.

\subsubsection{Pion decay constant}

Recently, Kneur and Neveu have used Optimized Perturbation Theory (see Section 3.8), supplemented by RG relations, to compute the dimensionless ratio of the pion decay constant $f_{\pi}$ to $\Lambda$ [128]. The modified optimized perturbative calculations allow to access the strong $\alpha_{s}$ regime and to account for QCD's dynamical chiral symmetry breaking characterized by $f_{\pi}$. The perturbative series for $f_{\pi} / \Lambda$ is known to fourth order: $f_{\pi}$ is defined from the vacuum expectation of the autocorrelation function of the axial current. It has been computed perturbatively up to fourth order. Relating the mass scale in this perturbative series to $\Lambda$ yields $f_{\pi} / \Lambda$, which reads at first order and in the chiral limit:

$$
\Lambda^{L O}=f_{\pi} / \sqrt{5 /\left(8 \pi^{2}\right)},
$$

that is $f_{\pi} / \Lambda \simeq 0.25$ in the $\overline{M S}$ scheme. The high order numerical calculations depend on the assumed value of $n_{f}$. The results calculated at different orders are stable. The fourth order result for $n_{f}=3$ yields $\Lambda_{\overline{M S}}=0.317 \pm 0.013 \mathrm{GeV}$, after correcting for chiral symmetry breaking. This correction produces a 10-20\% decrease of $\Lambda_{\overline{M S}}$ depending on $n_{f}$ as assessed from lattice results. This yields a coupling $\alpha_{s}\left(M_{Z}^{2}\right)=0.1174_{-0.0012}^{+0.0015}$.

As is the case of the holographic QCD approach discussed in the previous section, it is expected that QCD predictions can only provide dimensionless ratios $\Lambda_{\overline{M S}} / M$ or $\Lambda_{\overline{M S}} / f_{\pi}$ since the units of dimensionful quantities, meters or $\mathrm{eV}$ are arbitrary (human convention). 


\subsubsection{Grand unification}

Precise measurements of the fundamental force couplings offer a way to investigate the physics beyond the Standard Model [129]. The merging of the couplings in supersymmetric extensions of the Standard Model provides a theoretical prediction, $\alpha_{s}\left(M_{Z}^{2}\right)=0.129 \pm 0.010$ [130], although due to threshold effects, the runnings of the couplings have to be treated with care [30]. A prediction from the minimal $\mathrm{SU}(5)$ supersymmetric extension of the Standard Model in 5-dimensions yields a prediction closer to the measurements, $\alpha_{s}\left(M_{Z}^{2}\right)=0.118 \pm 0.005[131]$.

\subsubsection{Comparison and discussion}

Recent world data compilations -together with the latest extractions of $\alpha_{s}\left(M_{Z}^{2}\right)$ not included in those compilations [132]- are shown on Fig. 3.6. We also highlight the most accurate individual determinations.

This plot is only meant to be representative, rather than exhaustive, of the recent and most accurate measurements of $\alpha_{s}$. Fitting this choice of individual determinations (excluding one of the two DIS highly correlated determinations) yields a $\chi^{2} / n d f$ of 4.3, significantly larger than 1 . This demonstrates the tensions between the different determinations. Using the Unbiased Estimate, the average value of the coupling is $\alpha_{s}\left(M_{Z}^{2}\right)=0.1171 \pm 0.0006$. However, since we used only an arbitrarily selected sample of the available $\alpha_{s}$ determinations, this number should not be taken as a world average or compared to such averages. The $\chi^{2} / n d f$ values from other compilations before applying the Unbiased Estimate procedure also underline these tensions. In particular, there are clearly two classes of results differing by about $4 \%$. The lower class of values comes from DIS measurements, $\tau$-decay and thrust measurements. The Unbiased Estimate is certainly not a flawless compilation procedure since it does not account for possible correlations between results. While the question of how to best combine all the data remains difficult, it is likely that the large $\chi^{2} / n d f$ values are due to a tendency to underestimate uncertainties rather than inadequate compilation procedures, indications of physics beyond the Standard Model, or of a failing of QCD. Indeed, the consistency within a few percent between all determinations is a crucial verification of the validity of QCD. 


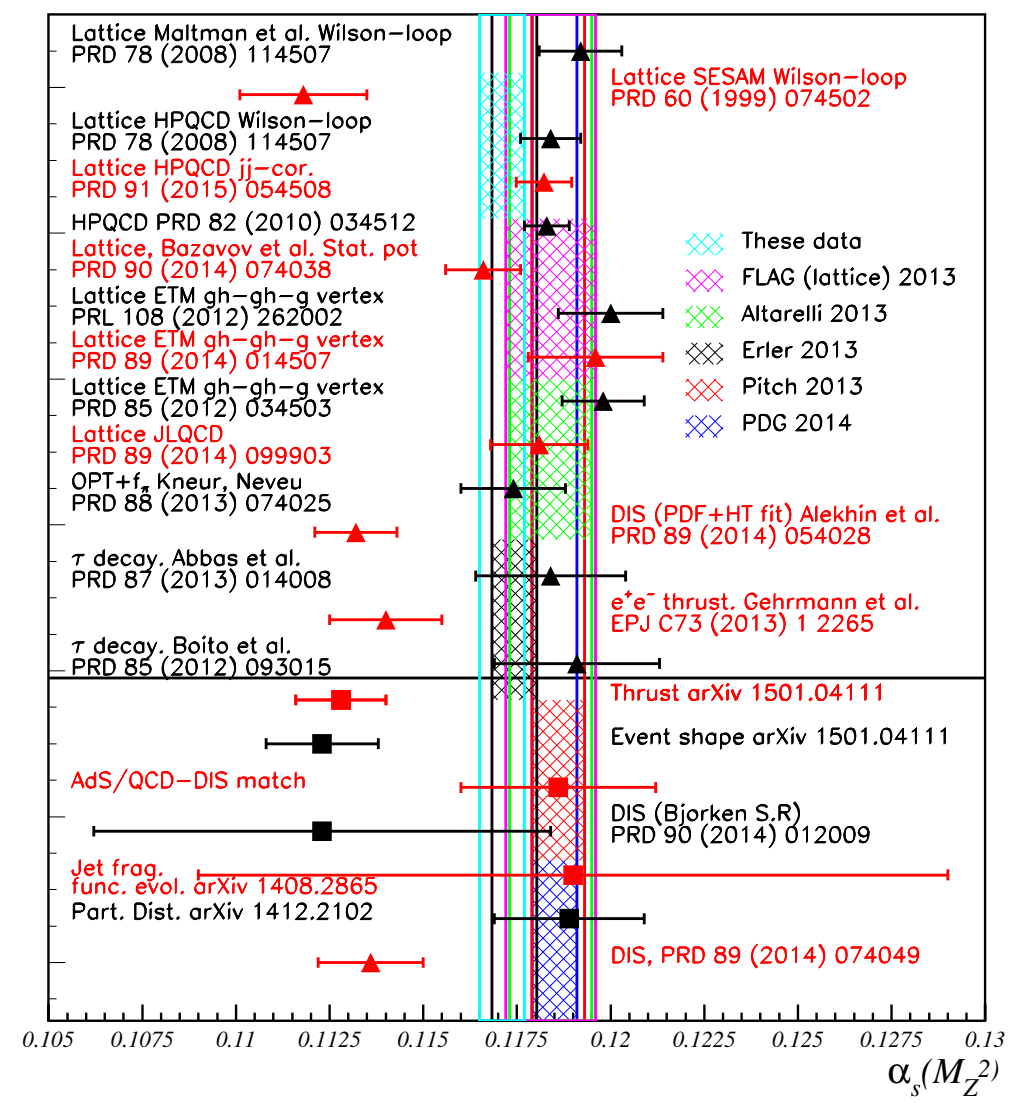

Figure 3.6: The coupling $\alpha_{s}\left(M_{Z}^{2}\right)$. Global averages from the most recent compilations of the world data are shown by the vertical bands. Also shown are recent determinations of $\alpha_{s}\left(M_{Z}^{2}\right)$ not yet included in the 2013-1014 global averages (squares), and some of the most accurate available determinations of $\alpha_{s}\left(M_{Z}\right)^{2}$ (triangles). Results computed at $n_{f}<5$ are corrected to $n_{f}=5$. 


\section{Chapter 4}

\section{The strong coupling in the nonperturbative domain}

As mentioned in the Preamble, knowing the strong coupling in the nonperturbative domain is necessary to understand both high energy and hadronic phenomena. An example is the calculation of the Sivers function [133] encountered in single-spin pseudoT-odd semi-inclusive DIS. In addition, the renormalization scale at arbitrarily small momenta $q^{2} \sim v^{2} S$ is required to evaluate heavy quark production as one approaches threshold [134]. Finally, knowing the IR-behavior of $\alpha_{s}$ is important to understand the mechanisms for dynamical chiral symmetry breaking [135]. The following references provide other examples of phenomena for which knowing the IR-behavior of $\alpha_{s}$ is required: $[136,137,138,139,140,141,142]$. In addition, the review [143] provides a concise description of the impact of $\alpha_{s}$ on our understanding of hadron dynamics. Additional reviews on $\alpha_{s}$ in the IR regime are given in $[2,144,145]$.

Studying $\alpha_{s}$ in the IR is more challenging than in the UV domain since the perturbative formalism involving the fundamental QCD fields cannot be used. Consequently, a number of approaches have been explored. Each approach has its benefits, justifications and limitations. In this second part of this review, we will explore the insights brought by the various approaches. Since there is still no consensus on how $\alpha_{s}$ should be defined, such discussion is necessary for several reasons: 1) the different definitions, and consequently meanings, of $\alpha_{s}$ need to be understood; 2) the approximations used and the arbitrary choices made (e.g., the RS and gauge) need to be noted; 3 ) the connections between the different approaches need to be identified; 4) it illustrates how this challenging problem can be approached by a variety of methods; 5) it acknowledges the theoretical contributions, early or recent, toward solving the problem of the IR-behavior 
of $\left.\alpha_{s} ; 6\right)$ finally, the variety of approaches to $\alpha_{s}$ in the IR domain can allow a cross-check of the conclusions.

Although there are important results and constraints obtained from experiments, most investigations of the IR-behavior of $\alpha_{s}$ have been theoretical. Some of the insightful theoretical approaches address theories which are closely related to QCD, including pure gluonic Yang-Mill theory (without quark fields), spaces of different Minkowsky or Euclidian dimensions, as well as $\mathrm{SU}\left(N_{C}\right)$ with various numbers of colors $N_{C}$. In that last context, it is useful to note that one recovers Abelian theory in the limit $N_{C} \rightarrow 0$ at fixed $\alpha_{s} C_{F}=\alpha$ where $C_{F}=\frac{N_{C}^{2}-1}{2 N_{C}}[147]$.

Perturbative QCD, via Eq. (3.24), evidently predicts that $\alpha_{s}\left(Q^{2}\right)$ diverges at the Landau pole, when $Q^{2} \rightarrow \Lambda^{2}$. However, this is not a meaningful prediction since it occurs, by definition, outside the domain of pQCD's validity. As we will discuss in Section 5.3 , the absence of nonperturbative terms in the series (3.24) is responsible for this unphysical prediction. Consequently, the Landau singularity cannot be cured by simply adding more perturbative terms to Eq. (3.24). In fact, they can worsen the situation; for example, a typical quantum field theory perturbative approximant is a Poincaré series (asymptotic series) which diverges beyond the order given approximately by the inverse of the expansion coefficient [148]. At $Q^{2}=1 \mathrm{GeV}^{2}$, this is typically $\alpha_{s} / \pi \simeq 0.2$. Thus, the perturbative approximant of an observable will start diverging after $n \simeq 5$. Since the $\beta$-series is expanded in powers of $\hbar$, this discussion does not directly concern its convergence, except that one traditionally chooses the $\beta_{i}$ order in Eq. (3.24) to be the same as the $\alpha_{s}$ order in the approximant series. Thus, the $\beta_{i}$ and $\alpha_{s}$ orders are linked.

It is often heard that the diverging behavior of the perturbatively calculated $\alpha_{s}$ is responsible for quark confinement. This ignores the fact that the Landau pole is unphysical and that Eq. (3.24) does not include important diagrams which connect quarks via multi-gluon exchange such as the "H" diagrams, see Fig. 2.2d. Such diagrams must be included for large distances calculations. Nevertheless, one can consider the possibility that $\alpha_{s}$ diverges as $1 / Q^{2}$ in the $Q^{2} \rightarrow 0$ limit, or one can argue that a large value for the coupling is an ingredient for confinement, but confinement does not require $\alpha_{s}$ to diverge. For example, according to lattice gauge theory simulations, the static $\mathrm{Q}-\overline{\mathrm{Q}}$ potential continues to rise linearly well after the coupling has reached a maximum value, typically at distances larger than $0.4 \mathrm{fm}$. The predicted QCD potential continues to rise linearly for Q- $\bar{Q}$ separations of order $1.3 \mathrm{fm}$ [150] or even larger distances for quenched calculations [151]. Furthermore, the IR value of $\left\langle\alpha_{s}\right\rangle=\frac{1}{E} \int_{0}^{E} d Q \alpha_{s}\left(Q^{2}\right)$ obtained from analyzing jet shape observables in $e^{+} e^{-}$annihilation and DIS is finite and typically 
modest: $\left\langle\alpha_{s}\right\rangle=0.47 \pm 0.07$ for $E=2 \mathrm{GeV}$ [152]. In the stochastic vacuum model approach to high-energy scattering [153], it is found that $\alpha_{s} \simeq 0.81$ in the IR. In another approach, Gribov showed that light quarks are super-critically bound if the averaged coupling has a moderate value of 0.43 at large distances [154].

Most definitions of $\alpha_{s}$ in the IR attempt to generalize the pQCD definition to include QCD's confining effects. Thus, the question of the IR-behavior of $\alpha_{s}$ is intimately linked to the topic of confinement. Consequently, confinement needs also to be addressed here. In the approaches that we will discuss, $\alpha_{s}$ is defined from the behavior of quark, gluon, and/or ghost propagators which are believed to be directly relevant to confinement. Such definitions of $\alpha_{s}$ are used in the Schwinger-Dyson framework (Section 4.4), the lattice technique (Sections 3.9.4 and 4.5), the Gribov-Zwanziger approach (Section 4.7), the Functional Renormalization Group framework (Section 4.6) and Stochastic Quantization (Section 4.8).

A popular picture of confinement, first put forth by Gribov [155] and then developed by Zwanziger (Section 4.7), is supported by the different framework just mentioned. It is useful to have such confinement picture in mind in order to understand the implications of the IR-behavior of $\alpha_{s}$. In the Gribov-Zwanziger scenario, the gluon propagator is IR-suppressed compared to an extrapolation of the $1 / Q^{2} \mathrm{UV}$-behavior. Meanwhile, the ghost propagator is IR-enhanced. Consequently, in this picture, confinement results from the long-distance propagations of ghosts. Thus, the behavior of the ghost and gluon propagators dictate the IR-evolution of $\alpha_{s}$.

Since ghosts are unphysical artifacts reflecting gauge choices and introduced to force the gluon propagator to be transverse (see Eqs. (3.28) and (3.29) and the discussion in between), a more intuitive but equivalent view is provided by the Stochastic Quantization framework in which there are no ghosts. Their role is played by longitudinal gluons in this formalism (see Section 4.8). At distances close to a color charge where pQCD rules and where spherical symmetry is not yet disturbed by the presence of another color charge, short wavelength gluons can be represented by plane waves (on-shell; i.e., transversely polarized gluons). At distances relevant to the large distance separation of the two color charges, the gluon field becomes highly asymmetric and is postulated to collapse into a flux tube. Consequently, the plane waves are fully distorted and longitudinally-polarized off-shell gluons dominate. Furthermore, it was shown that the ghost propagator (a manifestation of longitudinal gluons) in Landau gauge, $\partial_{\mu} A^{\mu}=0$, is related to the instantaneous Coulomb propagator in the Coulomb gauge, $\nabla \cdot \mathbf{A}=0$. The Coulomb propagator is thus enhanced at large distance [156], which leads to a linear 
Q- $\bar{Q}$ potential [157, 158, 159]. A dominating longitudinal gluon propagator indicates off-shell gluons, and thus the concept of effectively massive gluons becomes relevant (see Section 4.4.4).

This picture of confinement implies a specific IR-behavior of $\alpha_{s}$, namely that $\alpha_{s}$ loses its $Q^{2}$-dependence in the IR regime. In other words, it effectively has a conformal behavior, freezing at a given value: an infrared fixed point. Such behavior appears to be supported by measurements and calculations using different techniques as we will see in the rest of the review.

However, the Gribov-Zwanziger scenario described above has been challenged, as will be further discussed in Section 4.4.3. In fact, as we already said, there is no agreement on the IR-behavior of $\alpha_{s}$. The only certitude is that the Landau pole is unphysical. Rather than validating the conformal scenario, some studies indicate that $\alpha_{s}\left(Q^{2}\right)$ reaches its maximum in the IR-UV transition region and then vanishes in the deep IR. Other investigations point to a divergence of $\alpha_{s}\left(Q^{2}\right)$ when $Q^{2} \rightarrow 0$, where this divergence is unrelated to the Landau pole.

There are several reasons for these different conclusions. First and foremost, multiple definitions of $\alpha_{s}\left(Q^{2}\right)$ in the IR exist. Second, approximations are not always under control, and thus they may lead to unphysical artifacts. Third, there is no agreement on the more suitable form (instant form or front form) [123], gauge, and RS to use. Finally, since some of the results are model-dependent, some assumptions on which the model rests may not be systematically valid.

In the next sections, we will review the different approaches used or developed to study the low IR-behavior of $\alpha_{s}$, as listed by the theoretical techniques. Then we will compare the various results and discuss their differences.

First, we will briefly expand the discussion on the possible types of IR-behaviors for $\alpha_{s}$. The possibility that $\alpha_{s}\left(Q^{2}\right)$ loses its $Q^{2}$-dependence in the IR; i.e., $\beta\left(Q^{2}\right) \rightarrow 0$ in the IR domain, was pointed out in the early days of QCD [16, 160, 161]. A general argument, given in Refs. [5, 6], provides a physical explanation: color confinement implies that long wavelengths of partons in hadrons are cut off. Consequently, at this maximum wavelength corresponding to the typical hadron size, the effects of loops in propagators and vertex corrections disappear. Since these quantum effects are at the origin of the running of $\alpha_{s}$, it should freeze at the typical hadronic scale, provided that no other phenomena are included in the IR-definition of $\alpha_{s}$ : an infrared fixed point becomes a natural consequence of confinement. In fact, a number of theories and models that include confinement effects produce this feature, but their predictions of $\alpha_{s}$ do not freeze 
at a same value, and/or at the same momentum scale. The concept that $\alpha_{s}$ freezes is important since it allows one to compute quantities involving integrals over the IR domain of the coupling constant. It also permits the use of conformal field theory for nonperturbative QCD calculations. This connects to the AdS/CFT approach (Sections 3.9.6 and 4.2), including the extension of the CSR (Section 3.7.1 and Refs. [162] and [163]). In fact, the success of the AdS/CFT predictions may be an indication that $\alpha_{s}$, with a definition relevant to IR phenomenology, freezes in the IR. Experimental measurements of effective charges, results from lattice gauge theory, the Schwinger-Dyson formalism, the phenomenology of the hadron mass spectrum, the Gribov-Zwanziger confinement scenario and other approaches, also support this behavior.

Alternatively, the QCD running coupling may diverge as $1 / Q^{2}$, as suggested by the behavior of the $\mathrm{Q}-\overline{\mathrm{Q}}$ static potential at large distances. Yet another possibility is a monotonic increase of $\alpha_{s}\left(Q^{2}\right)$ as $Q^{2}$ becomes small, but without any divergence; i.e. $\beta\left(Q^{2}\right)$ remains non-zero and significantly negative. This is exemplified by the "analytic coupling", see Section 4.9.1. Finally, $\alpha_{s}$ may vanish in the IR. Several models, see e.g. Refs. $[152,164,165]$ and some Schwinger-Dyson and Lattice results indicate such behavior. Some experimental results [166] are also suggestive of this behavior.

In the remaining part of this review, we will use $\alpha_{s}$ as a generic designation for the strong coupling, and $\alpha_{* * *}$ for specific couplings, where "*** indicates the method, authors or schemes, defining $\alpha_{s}$. We will start by discussing effective charges and related definitions such as the coupling from holographic QCD and Sudakov charges. Then we will discuss the coupling from the static $\mathrm{Q}-\overline{\mathrm{Q}}$ potential and the information that the hadron spectrum provides on the IR-behavior of $\alpha_{s}$. The Schwinger-Dyson, lattice, functional renormalization group, Gribov-Zwanziger and stochastic quantization approaches will be discussed thereafter. These approaches use the same definition of the coupling. Finally, we will describe other frameworks, the most developed being the analytic/dispersive approaches.

Although the different approaches are organized in different sections for the sake of clarity, there are of course interrelations. For example, the effective coupling of Dokshitzer et al. (Section 4.9.2) is related to the effective charge approach of Grunberg (Section 4.1) and to the Shirkov et al. analytic approach (Section 4.9.1). Dokshitzer's coupling can be interpreted in terms of effectively massive gluon fields, which ties it to the Schwinger-Dyson framework (Section 4.4), the lattice results (Section 4.5) and other approaches addressing the IR-behavior of $\alpha_{s}$, since IR-regularizations are often associated with the emergence of a mass scale. The relevant mass scale can be interpreted 
as:

- the mass scale characterizing the harmonic oscillator potential in the light-front Schrödinger equation underlying quark bound states. Equivalently, it is also the factor distorting the AdS space in AdS/QCD (Section 4.2);

- the QCD string tension $\sigma$ (Section 4.3);

- an effective gluon mass (Sections 4.4.4, 4.5 and 4.9.1);

- a dispersive variable ("dispersive mass") (Section 4.9.2);

- the regulator which is introduced in the functional renormalization group method (Section 4.6);

- the Gribov mass (Section 4.7);

- a glueball mass (Sections 4.9.1 and 4.10).

These scales are thus all related and typically take values of order $0.5 \mathrm{GeV} \simeq 1.5 \Lambda_{\overline{M S}}$. In addition, all of these approaches have to connect to the phenomenological $\mathrm{Q}-\overline{\mathrm{Q}}$ linear potential. While the existence of such relations is physically suggestive, the explicit connection between the various strong couplings $\alpha_{s}$ in the infrared is however often unknown.

\subsection{Effective charges}

As we have noted, Eq. (3.24) implies that $\alpha_{s}\left(Q^{2}\right)$, as derived from pQCD, diverges when $Q^{2} \rightarrow \Lambda^{2}$. However, observables measured across the domain extending from $Q^{2} \gg \Lambda^{2}$ to $Q^{2}<\Lambda^{2}$ display no sign of discontinuity or unusual behavior. This is expected since the Landau pole is unphysical and $\Lambda$ is an arbitrary quantity which depends on the choice of RS, see Table 3.1. In contrast, observables must be RSindependent. This continuity of the observables, along with Grunberg's effective charge approach, see Section 3.7.1, provides a definition of an effective coupling that behaves as $\alpha_{p Q C D}$ at large $Q^{2}$ but stays finite at small values of $Q^{2}$.

Effective charges $\alpha_{s}^{e f f}\left(Q^{2}\right)$ are defined directly from observables which are calculable in the pQCD domain. A prominent example, which we will discuss in detail in the following sections, is the effective charge $\alpha_{g_{1}}\left(Q^{2}\right)$ defined from the Bjorken sum rule 
[36]. This effective charge definition of the running coupling incorporates QCD contributions inherent to deep inelastic scattering, QCD quantum corrections, and also the $Q^{2}$ -dependent perturbative effects responsible for the higher order terms of the observable's perturbative series, e.g., gluon bremsstrahlung.

Since it extends to low $Q^{2}$, an effective charge $\alpha_{s}^{e f f}\left(Q^{2}\right)$ also incorporates nonperturbative QCD contributions. Those can be organized as power-law corrections and thus are often referred to as "higher-twist" contributions as classified by the operatorproduct expansion. For example, the effective charge defined from the integral in the Bjorken sum rule, $\sum_{i=2,3 \ldots}^{\infty} \mu_{2 i}^{p-n}\left(Q^{2}\right) / Q^{2 i-2}$, incorporates the leading twist series on the rhs of Eq. (3.40). The $\mu_{2 i}^{p-n}\left(Q^{2}\right)$ terms are themselves sums of kinematical or dynamical higher-twists of twist order up to $2 i$. These terms have the usual pQCD $Q^{2}$-logarithmic dependence associated with asymptotic freedom and also can be interpreted in terms of the confining force acting on the quarks [167]. Thus the loop effects responsible for the UV running, the pQCD effects responsible for the higher order DGLAP evolution [67], and the nonperturbative confining forces are all incorporated into the effective QCD charge $\alpha_{s}^{\text {eff }}\left(Q^{2}\right)$. In some cases $\alpha_{s}^{\text {eff }}\left(Q^{2}\right)$ can take values in the IR domain that would be unphysical for a quantity measuring the absolute strength of a force. It is e.g. the case for the $\alpha_{D}(Q=0)=-\pi$ where $D\left(Q^{2}\right)$ is the Adler function [38]. This is obviously not a problem for an effective quantity.

A natural choice for an effective charge, close to the standard Gell-Mann-Low definition of the QED running coupling [35] is the potential scheme ( $V$-scheme), where $\alpha_{V}\left(Q^{2}\right)$ is interpreted as the running coefficient of the $1 / Q^{2}$ potential acting between heavy quarks [7, 40]. However, as discussed in Section 3.7.1, $\alpha_{V}$ is affected by infrared divergences at three loops and higher which prevent it from being calculated perturbatively, even at high $Q^{2}$.

A related approach has been proposed by Dokshitzer et al. [168, 169]. As in the case of effective charges, Dokshitzer's approach also generalizes the QED Gell-Mann-Low coupling. It will be discussed in detail in Section 4.9.2. Dokshitzer's charge is intended to measure the effective interaction strength in the IR in order to extend the use of the fundamental QCD fields outside the UV domain. We will see that Grunberg's effective charge defined from the Bjorken sum rule, $\alpha_{g_{1}}$, may be interpreted in a similar manner. In distinction to the standard effective charge, Dokshitzer's effective coupling is built by imposing dispersion relation constraints on the coupling. The effective charge $\alpha_{g_{1}}$ is also constrained by these restrictions since dispersion relations applied to photoproduction cross-sections are the basis for deriving the Gerasimov-Drell-Hearn sum rule [170], and 
this in turn constrains the IR-behavior of $\alpha_{g_{1}}$. Thus, although the respective formalisms of $\alpha_{g_{1}}$ and Dokshitzer's effective coupling seem to be different, the two couplings have some commonalities.

\subsubsection{Measurement of the effective charge from the Bjorken sum rule.}

The effective charge $\alpha_{g_{1}}\left(Q^{2}\right)$, defined from the Bjorken sum rule, has been discussed for large $Q^{2}$ in Sections 3.7.1 and 3.9.1, where it was shown that $\alpha_{g_{1}}\left(Q^{2}\right)$ has a simple perturbative series which is known to high orders in the $\overline{M S}$ scheme. An important advantage of $\alpha_{g_{1}}\left(Q^{2}\right)$ is that data exist at low, intermediate, and high $\mathrm{Q}^{2}[70,171,172$, $173,174,175,176,177,178]$. In addition, rigorous sum rules dictate the behavior of $\alpha_{g_{1}}$ in the unmeasured $Q^{2} \rightarrow 0$ and $Q^{2} \rightarrow \infty$ regions. A third advantage is that the Bjorken sum is a non-singlet quantity, implying that some resonance contributions to the sum, such as the $\Delta(1232)$ resonance, cancel out. This has important consequences. For example, disconnected diagrams which are not easily computed on the lattice, do not contribute, thus leading to more reliable lattice gauge theory estimates. Similarly, the cancellation of the $\Delta(1232)$ makes chiral perturbation calculations more robust [179]. Finally, $\alpha_{g_{1}}$ is easier to interpret than other effective charges due to similarities between the $g_{1}$ and $V$-schemes; see Fig. 3.4 and the associated discussion. In effect, the effective charge $\alpha_{g_{1}}$ can be evaluated at any $Q^{2}$, and its intuitive interpretation makes it easy to compare with theoretical expectations.

The similarities between the $g_{1}$ and $V$-scheme have a physical origin: First, $g_{1}$ is extracted from inclusive reactions, and the implicit sum over all final states simplifies the theoretical expression for the cross-section [180]. Second, the partial suppression of resonance contributions enhances the non-resonant background contribution. This allows DIS-like reactions to dominate the Bjorken integral even at low $Q^{2}$. In addition, the integral over $x_{f}$ and global parton-hadron duality [126] amplify the dominance of nonresonant reactions. In contrast, coherent state contributions are not easily interpreted in terms of a QCD coupling: Indeed, if an elastic contribution would be added to the definition of $\alpha_{g_{1}}$, it would become significantly negative because the kinematic constraint $\int_{0}^{1} d x_{f} g_{1}\left(x_{f}, Q^{2}\right) \rightarrow 0$ as $Q^{2} \rightarrow 0$ (see Eq. (4.2) below) would then not hold; this would yield $\alpha_{g_{1}+e l}\left(Q^{2}=0\right)=-17.6$, which includes the elastic contribution, rather than $\alpha_{g_{1}}\left(Q^{2}=0\right)=\pi$.

Two rigorous sum rules constrain $\alpha_{g_{1}}\left(Q^{2}\right)$ in the limits $Q^{2} \rightarrow 0$ and $Q^{2} \rightarrow \infty$ : the 


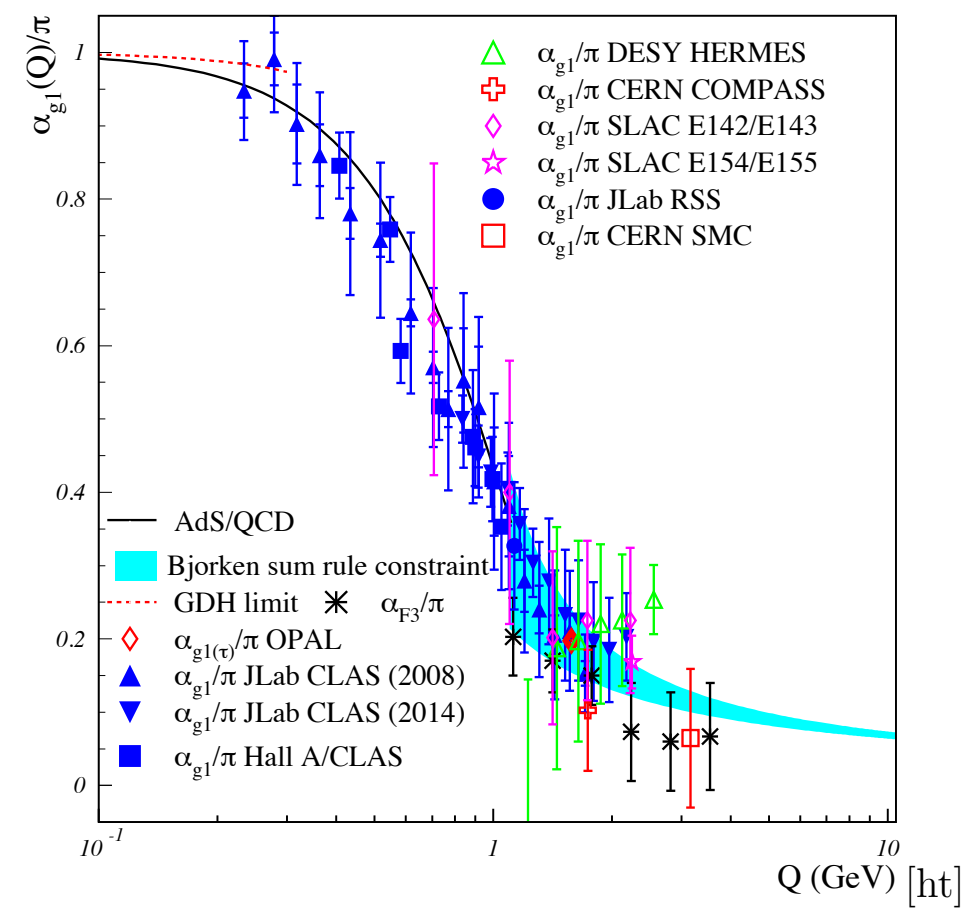

Figure 4.1: Experimental data and sum rule constraints for the effective charges $\alpha_{g_{1}}(Q) / \pi$ and $\alpha_{F_{3}}(Q) / \pi$. The blue data points are from Jlab [182], the green points are from Hermes [175], the black points are from Fermilab [72], the red points are from CERN [178] and the magenta points are from SLAC $[171,173,174,176]$.

Gerasimov-Drell-Hearn (GDH) sum rule [170, 181] for $Q^{2} \rightarrow 0$, and the Bjorken sum rule [36] for $Q^{2} \rightarrow \infty$. Let us first consider the latter. At large $Q^{2}$, the rhs of Eq. (3.40) can be computed. Equating it to the rhs of Eq. (3.41) yields the result:

$$
\alpha_{g_{1}}=\alpha_{\overline{M S}}+3.58 \frac{\alpha_{\overline{M S}}^{2}}{\pi}+20.21 \frac{\alpha_{\overline{M S}}^{3}}{\pi^{2}}+175.7 \frac{\alpha_{\overline{M S}}^{4}}{\pi^{3}}+\mathcal{O}\left(\alpha_{\overline{M S}}^{5}\right),
$$

which is indicated by the blue band shown in Fig. 4.1. The width of the band represents the uncertainties due to the value of $\Lambda_{\overline{M S}}$, the truncation of the Bjorken series in Eq. (4.1), and the truncation of the $\beta$ series used to compute $\alpha_{\overline{M S}}$ in Eq. (4.1). At the smallest $Q^{2}$ typically considered for the applicability of pQCD, $Q_{\min }^{2} \gtrsim 1 \mathrm{GeV}^{2}$, the asymptotic series (4.1) converges up to order $n \sim \pi / \alpha_{\overline{M S}}\left(Q_{\text {min }}^{2}\right) \simeq 4$ so one should stop at this order, lest $175.7 \alpha_{\frac{4}{M S}} / \pi^{3}$ becomes comparable to $\mathcal{O}\left(\alpha \frac{5}{M S}\right)$.

As an alternative to Eq. (4.1), one can use the BLM/PMC expression (see Section 3.7.1 and Eq. (3.43)) for $\alpha_{g_{1}}\left(Q^{2}\right)$ in terms of $\alpha_{\overline{M S}}\left(Q^{2}\right)$ [50]. In this case all nonzero $\beta$ terms are shifted into the scales $Q^{*}, Q^{* *}, \ldots$ of the $\alpha \overline{M S}$ coupling thus matching a 
conformal expansion. However, the domain of validity of this particular relation -a $C S R$ - may not be useful due to the large decrease in the scales $Q_{\overline{M S}}^{*}=0.37 Q_{g_{1}}^{*}$ and $Q_{\overline{M S}}^{* * *}=0.25 Q_{g_{1}}^{* * *}$. This decrease renders the computation of $\alpha_{\overline{M S}}$ problematic.

Let us consider now the GDH sum rule [170] which can be expressed as the limit:

$$
\frac{8}{Q^{2}} \int_{0}^{1^{-}} d x_{f} g_{1}\left(x_{f}, Q^{2}\right) \underset{Q^{2} \rightarrow 0}{\longrightarrow} \frac{-\kappa_{N}^{2}}{M_{N}^{2}}
$$

where $\kappa_{N}$ is the anomalous magnetic moment of the target particle (here a nucleon) and $M_{N}$ its mass. The elastic scattering contribution to $g_{1}, x_{f}=1$ is excluded from the integral in Eq. (4.2). At $Q^{2}=0$, there is no elastic reaction and at large $Q^{2}$ it is negligible, thus its exclusion is unimportant. At intermediate $Q^{2}$, the elastic contribution would be sizable if one chooses to include it to Eq. (3.41) (in that case, Eq. (4.2) becomes invalid). However, as already discussed, this contribution should be excluded for $\alpha_{g_{1}}$.

Eqs. (4.2) and (3.41) provide an important constraint on $\alpha_{g_{1}}$ at $Q^{2}=0$ :

$$
\left.\frac{d \alpha_{g_{1}}\left(Q^{2}\right)}{d Q^{2}}\right|_{Q^{2}=0}=\frac{3 \pi}{4 g_{A}}\left(\frac{\kappa_{n}^{2}}{M_{n}^{2}}-\frac{\kappa_{p}^{2}}{M_{p}^{2}}\right) .
$$

Furthermore, there is an additional kinematic constraint, $\int_{0}^{1^{-}} d x_{f} g_{1}\left(x_{f}, Q^{2}=0\right)=0$, because when $Q^{2}$ is small, only the elastic scattering contribution is permitted. However, the exclusive reaction is excluded from Eq. (3.41) leading to a vanishing integral at $Q^{2}=0$. This result also follows from Eq. (4.2). Consequently, the infrared fixed-point value

$$
\alpha_{g_{1}}(0)=\pi
$$

follows from (3.41).

The two constraints given by Eqs. (4.3) and (4.4) are shown in Fig. 4.1 by the dashed red line. The range between the low and high $Q^{2}$ constraints is densely filled by experimental data [182], mostly coming from the Bjorken integral data [70, 171, 172, $173,174,175,176,177,178]$. Other data come from the CCFR measurement [72] of the Gross-Llewellyn Smith sum rule [69], Eq. (3.47). At leading twist, the GLS sum rule has the same $Q^{2}$-dependence as the Bjorken sum rule, except for a small difference at order $\alpha_{\overline{M S}}^{3}$ coming from the light-by-light contribution to the GLS sum rule. Consequently, we expect in the perturbative domain that $\alpha_{g_{1}}\left(Q^{2}\right)=\alpha_{F_{3}}\left(Q^{2}\right)$ up to $\mathcal{O}\left(\alpha_{\overline{M S}}^{3}\right)$, with $\alpha_{F_{3}}$ defined from $\int_{0}^{1^{-}} d x F_{3}\left(x, Q^{2}\right) \equiv 3\left[1-\alpha_{F_{3}} / \pi\right]$. In addition, the kinematic constraint leading to Eq. (4.4) also applies to the GLS integral and thus $\alpha_{F_{3}}(0)=\pi=\alpha_{g_{1}}(0)$. In 
addition, as shown in Fig. 4.1, one can also use the value of $\alpha_{g_{1}(\tau)}$, which is obtained using a $C S R$, with the effective charge $\alpha_{\tau}$ defined from hadronic $\tau$ lepton decay, as reported in Ref. [39]. One also notes that the JLab data at low- $Q^{2}$ tend toward $\alpha_{g_{1}} \simeq \pi$, suggesting that $\alpha_{g_{1}}$ becomes nearly flat and thus "conformal" at $Q \lesssim 0.03 \mathrm{GeV}$.

A prediction of QCD's conformality from the GDH sum rule The approximate isospin symmetry between the proton and neutron implies that $M_{p} \simeq M_{n}$. Although $\kappa_{p}$ and $\kappa_{n}$ differ in sign, the squares are similar in magnitude: $\kappa_{p}^{2}=3.21 \simeq \kappa_{n}^{2}=3.66$. Consequently, the slope of $\alpha_{g_{1}}\left(Q^{2}\right)$ near $Q^{2}=0$ as given by Eq. (4.3) is suppressed. For example, in the deep IR region, $\alpha_{g_{1}}$ is expected to decrease by only $\simeq 1 \%$ over the $Q^{2}$-span of $0.1 \mathrm{GeV}^{2}$, consistent with near-conformal behavior in the low- $Q^{2}$ region.

\subsubsection{Measurement of the effective charge defined from $e^{+} e^{-}$ annihilation.}

The ratio $R_{e^{+} e^{-}}(s)$ of cross sections for $e^{+} e^{-}$annihilation to hadrons divided by muon pair production, and the ratio of hadronic over muonic $\tau$-decay widths, as well as the Adler function $D\left(Q^{2}\right)$ [38], have each been used to define various effective charges $[50,52]$. They are closely related to each other by CSRs. For example, in the purely perturbative domain, the couplings from $\tau$-decay and $R_{e^{+} e^{-}}$ratios, $\alpha_{\tau}$ and $\alpha_{R}$ respectively, are predicted by the CSRs to obey:

$$
\alpha_{\tau}(s)=2 \int_{0}^{s} \frac{d t}{s}\left(1-\frac{t}{s}\right)^{2}\left(1+\frac{2 t}{s}\right) \alpha_{R}(t),
$$

where $s$ and $t$ are the Mandelstam variables. The coupling $\alpha_{\tau}(s)$ has been extracted from the OPAL experimental data [84] by Brodsky and collaborators [39], with the result that $\alpha_{\tau}(0) \simeq 7.0$.

\subsubsection{Sudakov effective charges}

As is the case for the effective charges just discussed above, the Sudakov effective charges introduced by Gardi and Grunberg [183] essentially fold perturbative corrections into the definition of the effective coupling. In the large- $x_{f}$ limit of inclusive structure functions, collinear soft multi-gluon emissions dominate the perturbative series. These effects induce large logarithmic corrections (Sudakov double logarithms) which increase 
with the order of the perturbative series. These corrections can be incorporated by exponentiating (resumming) the Mellin transform of the structure function, leading to an exponential $e^{-S}$ damping factor, where $S$ is the Sudakov factor proportional to $\alpha_{s} l n^{2}$. In principle, this analysis includes all the relevant perturbative contributions. Nonperturbative power corrections are not explicitly included in the Sudakov factor. However, $S$ arguably does depend on such corrections since they influence the perturbative series through IR renormalons. The sum of the Sudakov anomalous dimensions, which appear in the exponentiation, defines the Sudakov effective charge. Such charges generalize the coupling definition of Ref. [58]. Most important, the Sudakov effective charges have a universal (scheme/observable-independent) freezing value.

The definition of the Sudakov effective charge is illustrated by an example provided in Ref. [183]. The Mellin transform of the DIS structure function $F_{2}\left(Q^{2}, x_{f}\right)$ is:

$$
\hat{F}_{2}\left(Q^{2}, N\right)=\int_{0}^{\infty} d x_{f} x_{f}^{N-1} F_{2}\left(Q^{2}, x_{f}\right)
$$

where $\hat{F}_{2}$ can be expressed, as usual, as a perturbative series in powers of $\ln \left(Q^{2}\right)$ which can be exponentiated. The argument of the exponentiated series is $\ln \left(\hat{F}_{2}\right)$. Differentiating it with respect to $\ln \left(Q^{2}\right)$ yields:

$$
\frac{d \ln \left(\hat{F}_{2}\right)}{d \ln \left(Q^{2}\right)}=\frac{16}{3}\left[H\left(Q^{2}\right)+\int_{0}^{1} d z \frac{z^{N-1}-1}{1-z} \mathcal{A}_{\mathcal{S}}\left[(1-z) Q^{2}\right]+\mathcal{O}(1 / N)\right]
$$

where $H\left(Q^{2}\right)$ and $\mathcal{A}_{\mathcal{S}}\left(Q^{2}\right)$ are given as perturbative series in powers of $\alpha_{s}$. In particular, $\mathcal{A}_{\mathcal{S}}\left(Q^{2}\right)=\frac{\alpha_{s}\left(Q^{2}\right)}{4 \pi}\left[1+A_{1} \alpha_{s}\left(Q^{2}\right)+\ldots\right]$ defines the Sudakov effective charge. It is connected to the two Sudakov anomalous dimensions $A$ and $B$ :

$$
\mathcal{A}_{\mathcal{S}}\left(Q^{2}\right)=\frac{3}{16}\left[A\left(\alpha_{s}\left(Q^{2}\right)\right)+\frac{d B\left(\alpha_{s}\left(Q^{2}\right)\right)}{d \ln \left(Q^{2}\right)}\right]
$$

In the large $Q^{2}$-limit, one has:

$$
\mathcal{A}_{\mathcal{S}}\left(Q^{2}\right)=\alpha_{s}\left(Q^{2}\right)+\frac{3}{4} \beta_{0}\left(\alpha_{s}\left(Q^{2}\right)\right)^{2}+\left(\frac{5}{4}-\frac{\pi^{2}}{3}\right) \beta_{0}^{2}\left(\alpha_{s}\left(Q^{2}\right)\right)^{3}+\cdots
$$

The IR limit is:

$$
\mathcal{A}_{\mathcal{S}}\left(Q^{2}\right)=\frac{1}{2 \beta_{0}}\left[1-\frac{2}{\pi} \arctan \left(\frac{\ln \left(Q^{2} / \Lambda^{2}\right)}{\pi}\right)+\frac{\Lambda^{2}}{Q^{2}}-\frac{\Lambda^{4}}{Q^{4}}\right],
$$


which has no Landau singularity, but it diverges toward negative values as $Q^{2} \rightarrow 0$. Nonperturbative power corrections may regularize this unphysical behavior. An example of a simple ansatz for the nonperturbative contributions, which can cancel divergences and lead to a $1 / \beta_{0}$ fixed point, is given in [184]. $\mathcal{A}_{\mathcal{S}}\left(Q^{2}\right)$ does not freeze in the IR, increasing from $1 / \beta_{0} \simeq 0.10$ at $Q^{2}=0$ toward a maximum at $Q^{2} / \Lambda^{2} \simeq 0.84$.

It was already noticed in [185] that the Landau pole can be suppressed by the Sudakov damping factor $e^{-S}$, although this suppression was not incorporated in the coupling discussed in Ref. [185] which retained the standard pQCD definition.

\subsection{AdS/CFT and Holographic QCD}

The behavior of the QCD coupling in the IR can be obtained by enforcing the underlying conformal symmetry of QCD. In fact, conformal symmetry even allows one to identify the form of the long-range color-confining potential by incorporating a method due to de Alfaro, Fubini and Furlan (dAFF), as discussed below. Its scale-dependence then leads to the running of the coupling in the IR as well as a constraint in the pQCD domain.

As we discussed in Section 3.9.6, light-front holographic QCD is based on the AdS/CFT correspondence [9] and the light-front quantization procedure based on the frame-independent front form devised by Dirac [123]. In the front form, the time evolution variable is $\tau=t+z / c$; i.e., the time along the light-front (LF). The front-form results are independent of the observer's Lorentz frame.

In this framework, one can reduce the full QCD light-front Hamiltonian equation to obtain a one-dimensional light-front Schrödinger Equation (LFSE) acting on the valence Fock state. The eigenvalues of the LFSE determine the hadron spectrum consisting of light quarks and the eigensolutions determine their LF wavefunctions. The LFSE is analogous to the Schrödinger equation describing hydrogenic atoms in QED [122] but it is relativistic and frame-independent. The radial variable for the LFSE is identified as the invariant distance between the $q$ and $\bar{q}: \zeta=b_{\perp} \sqrt{x(1-x)}$, where $\zeta^{2}$ is conjugate to the light-front kinetic energy $k_{\perp}^{2} /[x(1-x)]$. Here, $x$ is the light-front momentum fraction $x=k^{+} / P^{+}=\left(k^{0}+k^{z}\right) /\left(P^{0}+P^{z}\right)$ and $b_{\perp}$ is the transverse impact parameter.

If one requires the effective action which underlies the QCD Lagrangian to remain conformally invariant and extends the dAFF formalism to light-front Hamiltonian theory $[186,187]$, the light-quark antiquark potential has the unique form of a harmonic oscillator potential $V\left(\zeta^{2}\right)=\kappa^{4} \zeta^{2}$, and a mass gap arises. The dAFF mechanism, origi- 
nally derived in the context of $1+1$ quantum mechanics, enables the emergence of a mass scale $\kappa$ while the action remains conformal. The result is a nonperturbative relativistic light-front wave equation which incorporates confinement and other essential spectroscopic and dynamical features of hadron physics, including linear Regge trajectories with the same slope in the radial quantum number $n$ and orbital angular momentum $L$.

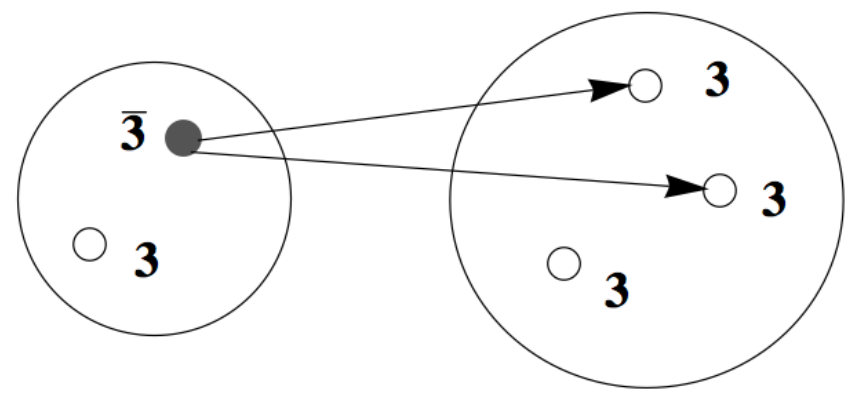

Figure 4.2: In QCD, hadronic dynamical supersymmetry is rooted in the dynamics of color $S U(3)$ where $\overline{\mathbf{3}} \sim \mathbf{3} \times \mathbf{3}$.

It has been shown recently how the main features of nonperturbative QCD dynamics are well captured in a semiclassical effective theory based on superconformal quantum mechanics [188, 189] and its extension to light-front physics [190, 191]. This new approach to hadron physics incorporates several basic aspects one expects from QCD, namely confinement and the appearance of a massless pion in the limit of zero quark masses. The specific breaking of conformal invariance uniquely determines the effective confinement potential. The generalized supercharges connect the baryon and meson spectra to each other in a remarkable manner. The partner mesons and baryons have the same mass provided one identifies the relative orbital angular momentum $L_{M}$ of the $q \bar{q}$ mesons with the relative internal orbital angular momentum $L_{B}$ of the quark-diquark baryons in a cluster decomposition, where $L_{M}=L_{B}+1$ [190, 191]. Furthermore, this framework gives remarkable connections across the full light [192] and the full heavylight hadron spectra, where heavy quark masses break the conformal invariance, but the underlying supersymmetry still holds [193]. It is important to recall that in the context of hadronic physics the supersymmetric relations are not a consequence of supersymmetric QCD, at the level of fundamental fields, but an emergent dynamical supersymmetry from color $S U(3)_{C}$. This relies on the fact that in $S U(3)_{C}$ a diquark can be in the same color representation as an antiquark, namely a $\overline{\mathbf{3}} \sim \mathbf{3} \times \mathbf{3}$ [194, 195, 196] as illustrated in Fig. 4.2. 
The light-front harmonic oscillator potential corresponds to a linear potential for bound states of nonrelativistic heavy quarks in the usually employed instant-form of relativistic dynamics [197]. This links light-front QCD to lattice gauge theory as well as heavy-quark effective theory. The mass parameter $\kappa$ can be determined from a hadron mass; e.g. $\quad \kappa=M_{\rho} / \sqrt{2}$ or $\kappa=M_{p} / 2$ [124], from the scale $\Lambda$ controlling pQCD evolution, as discussed in Section 3.9.6, or from other experimental inputs such as the Bjorken sum $Q^{2}$-evolutions. Remarkably, all of these determinations are in good agreement.

The underlying conformal symmetry of QCD allows one to perform equivalent calculations in a higher dimensional curved space-time where the dual theory is weakly coupled and described by gravity. Mathematically, this dual representations is based on the isomorphism of the $S O(4,2)$ group of conformal transformations in physical spacetime with the group of isometries of $\mathrm{AdS}_{5}$ space. Because of this correspondence, one can identify the fifth-dimension coordinate $z$ of $\mathrm{AdS}_{5}$ space with the light-front coordinate $\zeta$. Light-front holography also implies that the potential $V\left(\zeta^{2}\right)=\kappa^{4} \zeta^{2}$ appearing in the meson LFSE can be obtained by modifying $\mathrm{AdS}_{5}$ space using a specific "dilaton profile" $e^{ \pm \kappa^{2} z^{2}}[124,198]$ (The plus sign dilaton profile is required in light-front holographic QCD [124]). The quark-antiquark binding interaction is thus related in AdS/QCD to the modification of the AdS space curvature [187]. The same harmonic oscillator potential also emerges from the application of the dAFF procedure to LF theory [186, 124].

Remarkably, one also finds that the same modification of the $\mathrm{AdS}_{5}$ action also determines the IR-behavior of $\alpha_{s} \propto \exp \left(\frac{-Q^{2}}{4 \kappa^{2}}\right)$. Recall that in pQCD, the effective coupling $\alpha_{p Q C D}\left(Q^{2}\right)$ is defined by folding short-distance quantum effects into its evolution. Analogously, the $Q^{2}$-dependence of the AdS/QCD effective coupling stems from the effects of the large-distance forces folded into the coupling constant [162]. To obtain this result, one notes that the $\mathrm{AdS}_{5}$ action is similar to the action of general relativity: $S_{G R} \propto \int d^{4} x \sqrt{\operatorname{det}\left(g_{\mu \nu}\right)} R / G_{N}$, where $G_{N}$ is Newton's constant and $R$ is the Ricci scalar. In AdS/QCD, $\sqrt{R}$ is replaced by the gluon field $F, \sqrt{G_{N}}$ is replaced by the gauge coupling $g_{A d S}$, and the metric determinant becomes $\sqrt{\operatorname{det}\left(g_{A d S}\right)} e^{\kappa^{2} z^{2}}$, which includes the $e^{\kappa^{2} z^{2}}$ distortion of AdS space. This analysis yields the AdS action:

$$
S_{A d S}=-\frac{1}{4} \int d^{4} x d z \sqrt{\operatorname{det}\left(g_{A d S}\right)} e^{\kappa^{2} z^{2}} \frac{1}{g_{A d S}^{2}} F^{2} .
$$

Just as $\alpha_{s} \equiv g^{2} / 4 \pi$ acquires its effective $Q^{2}$ dependence from short-distance QCD quantum effects, the initially constant AdS coupling $\alpha_{A d S} \equiv g_{A d S}^{2} / 4 \pi$ is redefined to absorb the long distance confining forces; i.e. the effects of the modification of the AdS space 
curvature from nonconformal confinement dynamics [162]: $g_{A d S}^{2} \rightarrow g_{A d S}^{2} e^{-\kappa^{2} z^{2}}$. The five-dimensional coupling $g_{A d S}(z)$ is mapped, modulo a constant, into the QCD coupling $g_{s}$ of the confining theory in physical space-time using light-front holography. Thus, by identifying $z$ with the invariant impact separation variable $\zeta, g_{\operatorname{AdS}}(z) \rightarrow g_{s}(\zeta)$, one predicts

$$
\alpha_{s}^{A d S}(\zeta) \equiv g_{s}^{2}(\zeta) / 4 \pi \propto e^{-\kappa^{2} \zeta^{2}}
$$

The physical coupling measured at the scale $Q$ is the light-front transverse Fourier transform of the LF coupling $\alpha_{s}^{A d S}(\zeta)(4.12)$ :

$$
\alpha_{s}^{A d S}\left(Q^{2}\right) \sim \int_{0}^{\infty} \zeta d \zeta J_{0}(\zeta Q) \alpha_{s}^{A d S}(\zeta)
$$

in the $q^{+}=0$ light-front frame where $Q^{2}=-q^{2}=-\mathbf{q}_{\perp}^{2}>0$ and $J_{0}$ is a Bessel function. Using this ansatz we then have from Eq. (4.13)

$$
\alpha_{s}^{A d S}\left(Q^{2}\right)=\alpha_{s}^{A d S}(0) e^{-Q^{2} / 4 \kappa^{2}}
$$

where $\alpha_{s}^{A d S}(0)=\pi$ in the $g_{1}$ scheme. The identification of $\alpha_{s}^{A d S}\left(Q^{2}\right)$ with the physical QCD running coupling in its nonperturbative domain determines the space-time dependence at large distances of the physical four-dimensional coupling $\alpha_{s}$.

The harmonic oscillator form of the LF potential $V\left(\zeta^{2}\right)=\kappa^{4} \zeta^{2}$, and thus the Gaussian form for the running coupling, also follows from the dAFF procedure; i.e., the requirement that the action remains conformal [186, 124], even though a mass scale appears in the confining potential of the light-front Hamiltonian.

Eq. (4.14) is valid only at the domain of $Q^{2}$ where QCD is a strongly coupled theory and the AdS/CFT correspondence can be applied. Furthermore, since the semiclassical approximation used in the light-front holographic approach neglects quantum effects, vacuum polarization effects are not incorporated in $\alpha_{A d S}$, and consequently it is not valid at large $Q^{2}$. Nonetheless, $\alpha_{A d S}$ can be supplemented at large $Q^{2}$ by either parameterizing the well-known pQCD effects at the origin of the large $Q^{2}$ dependence, as done in [162], or by matching Eq. (4.14) to Eq. (3.24), as done in [125] and discussed in Section 3.9.6. This matching procedure is illustrated in Fig. 4.3.

The Gaussian form $\alpha_{s}\left(Q^{2}\right)=\alpha_{s}(0) e^{-Q^{2} / 4 \kappa^{2}}$ predicted by the light-front holographic approach describes the available experimental data for the effective charge $\alpha_{g_{1}}\left(Q^{2}\right)$ very well over a large range of moment transfer; see Fig. 4.1. Conversely, a fit of the experimental data shown on Fig. 4.1 with $\kappa$ as the free fit parameter yields $\kappa=0.496 \pm 0.007$, 


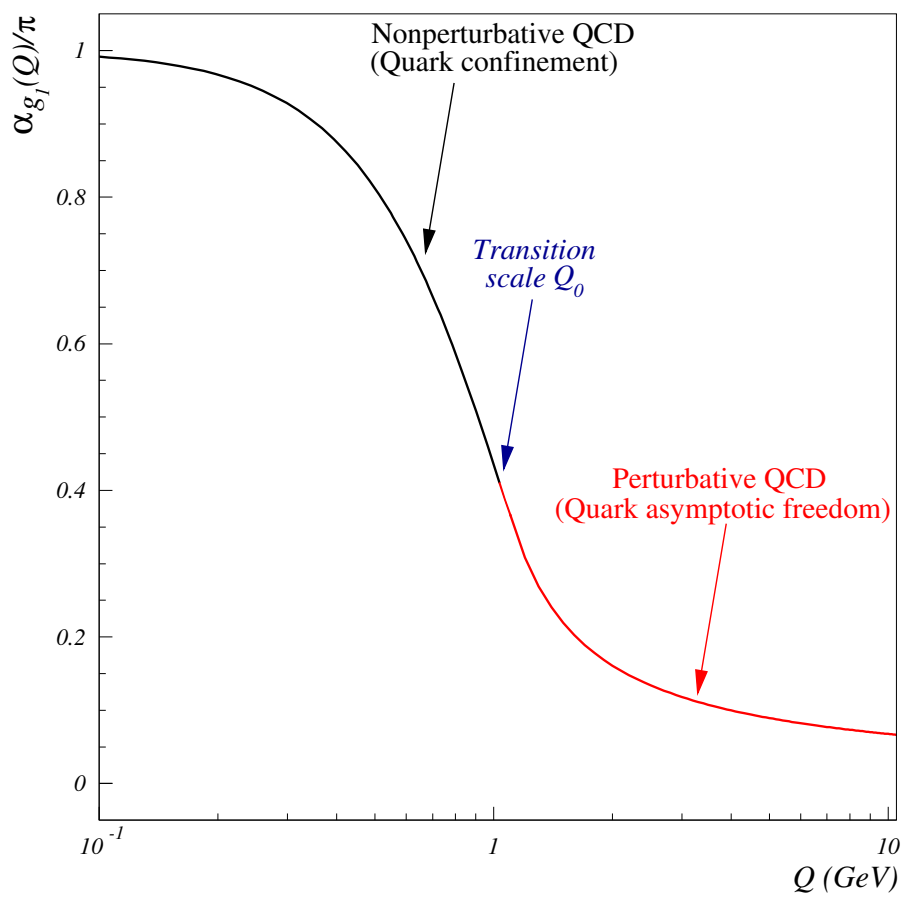

Figure 4.3: Unified coupling obtained from the analytic matching of nonperturbative and perturbative QCD regimes. The procedure determines the relation between $\Lambda_{\overline{M S}}$ and $\kappa$ or equivalently hadron masses. The transition scale $Q_{0}$ between the large and short-distance regimes of QCD is determined as well.

in good agreement with the determination from the value $\kappa=0.50 \pm 0.04$ obtained from the relation between $\kappa$ and hadron masses: $\kappa=M_{\rho} / \sqrt{2}$ or $\kappa=M_{p} / 2$ [124]. It is remarkable that the parameter $\kappa$ which fits the Bjorken sum rule data is set independently by a hadron mass.

Additionally, it has been shown using the Schwinger-Dyson equations for the quark propagator that if one assumes a Gaussian form for the QCD running coupling, the quark propagator has poles above and below the real axis, consistent with quark confinement [199]. Finally, the scaling solution of the Schwinger-Dyson equations yields a coupling in close agreement with $\alpha_{A d S}$, with a freezing value of 2.97 (Section 4.4). 


\subsection{The effective potential approach}

\subsubsection{The static $\mathrm{Q}-\overline{\mathrm{Q}}$ potential}

A remarkable feature of hadron physics is that mesons and baryons composed of light quarks all lie on Regge trajectories. The square of the hadron masses are linear in both the internal orbital angular momentum $L$ and the principal quantum number $n$ which is associated with the number of nodes in its wavefunction. Moreover the slope in $L$ and $n$ are identical phenomenologically. These universal features in fact follow from the eigenvalues of LFSE with the harmonic oscillator potential $V\left(\zeta^{2}\right)=\kappa^{4} \zeta^{2}$ [124]. The lightest eigenvalue, corresponding to the pion, has zero mass in the chiral limit $m_{q} \rightarrow 0$.

In the case of heavy quark $\mathrm{Q}-\overline{\mathrm{Q}}$ states, the effective nonrelativistic static potential between two heavy quarks $(\mathrm{Q}-\overline{\mathrm{Q}})$ of mass $m_{Q} \gg \Lambda$ separated by a distance $r$ is well described by:

$$
V(r)=-\frac{4}{3} \frac{\alpha_{V}(r)}{r}+\sigma r .
$$

The first term is a perturbative, Coulomb-type, one-gluon exchange contribution. The hadronic wavefunctions and the fine structure of the hadron spectrum are sensitive to this term. When $\alpha_{V}(r)$ is approximated as an averaged coupling $\left\langle\alpha_{V}\right\rangle$, Eq. (4.15) is known as the Cornell potential [200]. The mean value of the coupling $\left\langle\alpha_{V}\right\rangle$ depends on the size of the hadrons considered. Its value in $\mathrm{GeV}$ units spans from 0.19 to 0.4, with an optimal value of 0.22 [200, 201, 202]. In the context of an IR-freezing behavior of $\alpha_{V}\left(Q^{2}\right)$, this implies a freezing value of $\alpha_{V}(0)=0.42 \pm 0.03$ [200, 201, 202].

The second term in Eq. (4.15) is a linear confining potential which can be interpreted as the string tension between the $\mathrm{Q}-\overline{\mathrm{Q}}$ pair, with $\sigma \sim 0.18 \mathrm{GeV}^{2}$. This term controls the slopes and intercepts of the Regge trajectories. The QCD string picture was first postulated by Nambu, Nielsen and Susskind to interpret the Regge trajectories [203]. A common interpretation is that the string is formed by a chromoelectric flux tube and is responsible for quark confinement. Regge trajectories stem straightforwardly from the string picture: the faster a hadron spins, the larger the string tension must be to compensate for the centrifugal force, and hence the larger its mass [204].

In fact, the AdS/QCD holographic QCD framework which yields the harmonic oscillator potential $V\left(\zeta^{2}\right)=\kappa^{4} \zeta^{2}$ in the light-front transverse coordinate $\zeta$ for light quark pairs, corresponds to a linear potential for heavy quarks in the non-relativistic instantform radial coordinate $r$ for large distances [197]. The string tension for heavy quarks 
can then be predicted from holographic QCD to be:

$$
\sigma_{A d S}=2 \kappa^{2} / \pi \simeq 0.18 \mathrm{GeV}^{2}
$$

which is remarkably consistent with fits to the quarkonium data.

A more accurate potential than that provided by Eq. (4.15) includes velocitydependent and spin-dependent corrections, see Refs. [205] and [48] for reviews.

The validity of Eq. (4.15) is confirmed for heavy quarks by lattice QCD simulations. However, in $(2+1)$ lattice simulations, $\alpha_{V}(0) \sim 0.3$ [206], somewhat smaller than the value determined from the Cornell potential. Earlier quenched calculations resulted in an even smaller value of the coupling, $\alpha_{V}(0) \sim 0.22[206]$.

In addition to lattice QCD, other nonperturbative approaches also suggest the form of Eq. (4.15). In the Coulomb gauge, the instantaneous gluon propagator leads to

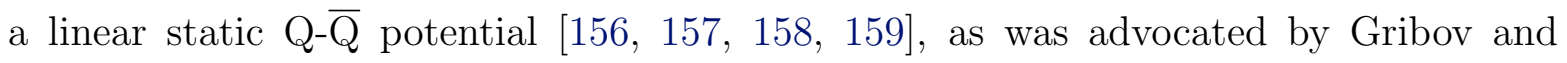
Zwanziger [155, 207]; see Section 4.7. Generally, it is expected that in the IR, the gluon propagator is modified by nonperturbative effects and displays a $1 /\left(Q^{2}+f\left(Q^{2}\right)\right)^{2}$ dipole form that would lead to the linear IR potential [208]. (The function $f\left(Q^{2}\right)$ is unimportant here. It is typically related to an effective gluon mass or a glueball mass.) This is studied in the Schwinger-Dyson formalism, see Section 4.4 and in particular Refs. [209, 210], and in the related approach of the functional renormalization group [211], see Section 4.6. A linear potential is also recovered using background perturbation theory [212], see Section 4.10 for details.

To summarize, Eq. (4.15) is well established. Nevertheless, there is no agreement on the IR-behavior of $\alpha_{s}$, owing to its various possible definitions. Still, within a specific definition, Eq. (4.15) -or more generally hadron spectroscopy- does constrain $\alpha_{s}$ as we will now discuss.

\subsubsection{Constraints on the running coupling from the hadron spectrum}

The hadron spectrum is sensitive to the IR-behavior of $\alpha_{s}$ when one identifies the strong coupling as the effective charge underlying the heavy $\mathrm{Q}-\overline{\mathrm{Q}}$ potential. Hence, the heavy quark potential can provide a useful information on $\alpha_{s}\left(Q^{2}\right)$ in the IR. For example in the Godfrey-Isgur quark/flux-tube model [213], $\alpha_{s}$ must freeze in the IR at $\alpha_{s}(0)=0.6$. Other examples are the model of Zhang and Koniuk [214], or the work of Badalian and collaborators using the framework of background perturbation 
theory. Badalian et al. obtain $\alpha_{s}(0)=0.58$ from the analysis of the bottomonium, see Section 4.10 and Ref. [215]. Likewise, Andreev [216] uses several of the forms for $\alpha_{s}\left(Q^{2}\right)$ suggested in the literature and constrains them with a Poincaré covariant quark model to reproduce meson characteristics and experimental data, including the Bjorken sum rule, Eq. (3.40), and the GLS sum rule, Eq. (3.47). Andreev obtains a consistent set of behaviors by using $\alpha_{s}\left(Q^{2}\right)$ defined in the $\overline{\mathrm{MS}}$ scheme, but regularized with a constant effective gluon mass using the massive analytic perturbation theory approach (Section 4.9.1), or with Cornwall's coupling (see Section 4.4.4) or with the "synthetic coupling" (also discussed in Section 4.9.1). This procedure results in a freezing value $\alpha_{s}(0) \simeq 0.71$ in the $\overline{\mathrm{MS}}$ renormalization scheme.

Baldicchi and Prosperi also tested several different expressions of $\alpha_{s}\left(Q^{2}\right)$ to check their compatibility with the meson spectrum [217]. One of the expressions studied is a crude freezing implementation: below a given $Q_{0}, \alpha_{s}$ is constant, and above $Q_{0}$ it follows the pQCD form. Another expression of $\alpha_{s}$ is given by the "analytic" approach described in Section 4.9.1 and given by Eq. (4.54). This $\alpha_{a n}$ is finite and decreases monotonously with $Q^{2}$. A related expression has been give by Dokshitzer et al., and is given by Eq. (4.59). Baldicchi and Prosperi conclude that the meson spectrum appears to be rather insensitive to the type of coupling used [217].

Historically, these approaches follow from the pioneering works of Richardson and of Buchmuller et al. . These authors suggested that $\alpha_{s}$ should be defined to incorporate a long range force in addition to the usual pQCD effects responsible for the running of the coupling $[218,219]$. In the non-relativistic (static) approximation, Eq. (4.15) becomes in momentum space:

$$
V(Q)=\frac{1}{(2 \pi)^{3}} \int d^{3} r e^{-i r \cdot Q} V(r)=-\frac{1}{\pi^{3}} \frac{2}{3} \frac{\alpha_{V}\left(Q^{2}\right)}{Q^{2}}-\frac{\sigma}{\pi^{3} Q^{4}} .
$$

Incorporating in the coupling the long distance $\sigma$-term leads to the definition of $\alpha_{\text {Rich }}\left(Q^{2}\right)$ :

$$
V\left(Q^{2}\right)=-\frac{1}{\pi^{3}} \frac{2}{3} \frac{\alpha_{\text {Rich }}\left(Q^{2}\right)}{Q^{2}}
$$

Richardson [218] proposed that:

$$
\alpha_{R i c h}\left(Q^{2}\right)=\frac{4 \pi}{\beta_{0} \ln \left(1+Q^{2} / \Lambda_{V}^{2}\right)},
$$

where $\Lambda_{V}$ should be used since $\alpha_{\text {Rich }}$ identifies to $\alpha_{V}$ in the UV. The corresponding 
$\beta$-function is:

$$
\beta=-\beta_{0} \alpha_{\text {Rich }}^{2}\left(1-e^{-1 /\left(\beta_{0} \alpha_{\text {Rich }}\right)}\right) .
$$

This expression anticipated results from the Schwinger-Dyson formalism; see Eq. (4.28), in which the coupling is regulated by an effective dynamical gluon mass $m_{g}\left(Q^{2}\right)$. Using Richardson's Eq. (4.19), the gluon mass is assumed to be constant and equal to $\Lambda_{V}$, which is close to the $m_{g}(0)$ values suggested by Schwinger-Dyson and lattice studies. The $Q^{2} \rightarrow 0$ limit of Eq. (4.19) is:

$$
\alpha_{\text {Rich }}\left(Q^{2}\right)=\frac{4 \pi \Lambda_{V}^{2}}{\beta_{0} Q^{2}},
$$

and the string tension

$$
\sigma=\frac{8 \pi \Lambda_{V}^{2}}{3 \beta_{0}}
$$

Using the phenomenological value $\sigma=0.18 \mathrm{GeV}^{2}$, one obtains $\Lambda_{V}=0.46 \mathrm{GeV}$ for $n_{f}=2$ in agreement with its high- $Q^{2}$ pQCD determination $\Lambda_{V}=0.48 \mathrm{GeV}$, see Table 3.1. Thus, if all the mechanisms leading to the long-distance linear potential are included in the definition of $\alpha_{V}$, then the $1 / Q^{4}$ divergence of $V$ in the instant-form coordinate system implies that $\alpha_{V}$ should diverge as $3 \sigma / 4 Q^{2}$ in the limit $Q^{2} \rightarrow 0$.

The fact that the linear $\mathrm{Q}-\overline{\mathrm{Q}}$ potential leads to $\alpha_{V} \propto 1 / Q^{2}$ has inspired several phenomenological models. Belyakova and Nesterenko [220] have constructed a coupling with the constraints that it follows $\alpha_{\overline{M S}}\left(Q^{2}\right)$ in the UV and $\alpha_{s}\left(Q^{2}\right) \simeq \frac{4 \pi}{\beta_{0}} \frac{\Lambda^{2}}{Q^{2}}$ in the IR. The Belyakova-Nesterenko coupling is defined by the $\beta$-function:

$$
\beta\left(\alpha_{B N}\right)=\beta_{\overline{M S}}^{(l)} \frac{1-e^{-8 \pi / \alpha_{B N} \beta_{0}}\left(1-l^{2} \beta_{0}^{l} / \beta_{l}\right)}{1+l^{2}\left(\alpha_{B N} \beta_{0} /(4 \pi)\right)^{l}} .
$$

It resembles the $\beta$-functions proposed in Refs. [218, 201]. At leading order, it yields:

$$
\alpha_{B N}\left(Q^{2}\right)=\frac{4 \pi}{\beta_{0}} \frac{1}{W_{0}\left(Q^{2} / \Lambda^{2}\right)}
$$

where $W_{0}$ is the principal branch of the real-valued Lambert function; see Section 3.2. The static Q- $\bar{Q}$ potential obtained for $\Lambda^{(3)}=375 \pm 40 \mathrm{MeV}$ agrees with both lattice QCD and the Cornell potential, Eq. (4.15). The model provides the same relation between $\Lambda$ and $\sigma$ found by Richardson, see Eq. (4.22). 


\subsection{The Schwinger-Dyson formalism}

Studies of the Schwinger-Dyson equation (SDE) for QCD have also provided important insights into the IR-behavior of $\alpha_{s}\left(Q^{2}\right)$. In effect, the SDE (and the corresponding Bethe-Salpeter formalism for bound states) incorporates an infinite set of coupled nonlinear equations relating Green's functions as well as other constraints from the QCD equations of motion. In this formalism, the equation for the $n$-point function depends on the equation for the $(n+1)$-point function. Although the SDE formalism can be described using perturbative Feynman graphs, infinite sets of diagrams are resummed into one-particle irreducible graphs, and thus the SDE can account for the nonperturbative content of the theory. Alternatively, the SDE can be derived using the generating functional of the Green's functions without reference to perturbative graphs. The SDE formalism thus stems from the fundamental equations of QCD, encompasses its nonperturbative effects, and is able in principle to provide analytical results. As such, it provides a powerful tool. Furthermore, unlike Lattice QCD, the SDE formalism is not subject to unphysical artifacts resulting from space-time discretization, nor unphysically large quark masses. These effects can complicate studies of important hadronic phenomena.

However, the SDE framework has its own difficulties: the infinite system of equations must be truncated to be amenable to solutions. In fact, any truncation of the kernel conflicts with crossing symmetry. In the case of QED, one must include an infinite number of crossed graph kernels in the Bethe-Salpeter equation in order to derive the Dirac Coulomb equation for muonium $\mu^{+} e^{-}$in the limit of $m_{\mu} \rightarrow \infty$. This implies that the SDE approach contains approximations which may lead to effects that are not easily understood and controlled. Also, the equations are difficult to solve and analytical answers can be hard to obtain without further approximations. Consequently, the SDE quantitative results are often obtained numerically. The SDE truncations are typically chosen to ensure that cut off propagators are positive (to retain unitarity), that the high- $Q^{2}$ behavior of the QCD coupling matches RGE expectations, and that the Green's functions retain their multiplicative renormalizability properties. The last property implies that a full propagator is proportional to its corresponding bare propagator via a "dressed function".

Despite the difficulties inherent to the SDE, this approach provides an important method for furthering our understanding of QCD in general and of $\alpha_{s}$ in particular. It is especially powerful when studied alongside other techniques which will be discussed in 
the next sections, noticeably lattice QCD, so that these methods can supplement each other. In these different approaches, couplings have the same definition and the $\mathrm{RS}$ is usually the same (the MOM RS or derivatives). Consequently, comparing quantities in SDE to other techniques is possible. As an example of the complementarity between the SDE framework and lattice approaches, the SDE can be used to understand the effects of space-time discretization and of choice of boundary conditions for the lattice approach, whereas the lattice can be used to understand the effect of truncating the infinite system of equations of the Schwinger-Dyson formalism. For general reviews on SDE see Ref. [221]. Reviews of SDE results discussing the IR-behavior of $\alpha_{s}$ can be found in $[222,223]$.

\subsubsection{The QCD coupling defined from Schwinger-Dyson equa- tions}

The behavior of the gluon and ghost Green's functions is directly related to the running of $\alpha_{s}$. The required propagators and vertices which define these Green's functions have been extensively studied using the Schwinger-Dyson formalism, on the lattice, and by using the Gribov-Zwanziger approach [155, 207], as well as other methods. See [224, 225] for recent reviews. For example, the strong coupling derived from the ghostgluon vertex $\alpha_{s}^{g h}\left(Q^{2}\right)$ is given in the Landau gauge and the MOM RS by

$$
\alpha_{s}^{g h}\left(Q^{2}\right)=\alpha_{s}^{g h}(\mu) G^{2}\left(Q^{2}, \mu\right) Z\left(Q^{2}, \mu\right)
$$

where $\alpha_{s}^{g h}(\mu)$ is the coupling determined at the renormalization scale $\mu, Z\left(Q^{2}, \mu\right)$ is the gluon propagator dressing function, and $G\left(Q^{2}, \mu\right)$ is the ghost propagator dressing function (the ghost propagator being the inverse of the Fadeev-Popov operator). The RG invariance of Eq. (4.25) is evident: the quantities forming the right-hand side depend on $\mu$, but on the left hand side, $\alpha_{s}^{g h}$ does not. The dressing functions are defined as $\delta^{b c} G\left(Q^{2}, \mu\right) / Q^{2}=G^{b c}\left(Q^{2}, \mu\right)$ and $-\delta^{b c} Z\left(Q^{2}, \mu\right)\left(\delta_{\mu \nu}-q_{\mu} q_{\nu} / q^{2}\right) / Q^{2}=D_{\mu \nu}^{b c}\left(Q^{2}, \mu\right)$ where $b, c$ are color indices, $G^{b c}\left(Q^{2}, \mu\right)$ is the ghost propagator, and $D_{\mu \nu}^{b c}\left(Q^{2}, \mu\right)$ is the gluon propagator. Since the dressing functions relate the first-order (i.e. bare) Green's functions to their fully (i.e. dressed) expressions, they are often called "form factors" in analogy with the functions modifying the point-like expression for elastic scattering due to hadron structure. There is no contribution from the vertex dressing function in Eq. (4.25) owing to the Slavnov-Taylor identity [32].

One sees from Eq. (4.25) that the analytic form of the propagators provides a 
definition of the strong coupling. Other definitions are possible using the three-gluon vertex (defining the coupling $\alpha_{s}^{3 g}$ ), the four-gluon vertex (defining $\alpha_{s}^{4 g}$ ) or the quarkgluon vertex (defining $\alpha_{s}^{q g}$ ). Since ghosts do not couple to themselves or to quarks, no further coupling definitions are possible.

The $\alpha_{s}^{3 g}, \alpha_{s}^{4 g}$ and $\alpha_{s}^{q g}$ definitions involve vertex dressing functions which are more difficult to study [22]. In particular, there are a large number of contributing Feynman graphs. Thus $\alpha_{s}^{g h}$ has been the focus of most of the initial SDE studies, although several groups have calculated the other couplings, The quantities $\alpha_{s}^{g h}, \alpha_{s}^{3 g}, \alpha_{s}^{4 g}, \alpha_{s}^{q g}$ are the same in a massless RS, such as $\overline{M S}$, but not for a massive RS such as the MOM RS [145]. Nevertheless, these couplings can be related to each other [22, 25, 145, 146], and the differences vanish at large $Q^{2}$. Another coupling used in the SDE is $\alpha_{\text {gse }}$, defined from the gluon self-energy [226]. It has been shown to be closely related to $\alpha_{s}^{g h}$ in the Landau gauge [227].

\subsubsection{Gauge choices}

Green's functions are not directly observable, and they become gauge-dependent if one utilizes the MOM RS. This also makes the derived results for $\alpha_{s}^{g h}, \alpha_{s}^{3 g}, \alpha_{s}^{4 g}$ and $\alpha_{s}^{q g}$ gauge dependent. An alternative procedure is to use Cornwall's Pinch scheme to define a gauge-independent three-gluon coupling [53, 228].

Most SDE results relevant to $\alpha_{s}$ are obtained in the Landau gauge, $\partial_{\mu} A^{\mu}=0$, since the relatively small numbers of fields and vertices makes computations of the SDE simpler relative to many other gauges. It is also a Lorentz-covariant gauge, which, beside making the Kugo-Ojima confinement criterion applicable, further simplifies solving the SDE. For example, of all the possible tensors (from bosons) or spinor (from fermions) terms which form the 2-point Green's function, only two survive generic symmetry requirements. They lead to a boson propagator that can be written as $A\left(Q^{2}\right) q^{2} \eta^{\mu \nu}-B\left(Q^{2}\right) q^{\mu} q^{\nu}$. These two functions, which are a priori independent, become evidently equal in Lorentz-covariant gauges such as the Landau gauge: $A\left(Q^{2}\right)=B\left(Q^{2}\right)$, yielding a propagator that depends only on $Q^{2}$. In contrast, gauges which are not covariant have propagators - and consequently predictions for $\alpha_{s}$ - which can depend on another variable in addition to $Q^{2}$. For example, in the first calculations of the gluon propagator done in the (non-covariant) axial gauge [229], $B\left(Q^{2}\right)$ was set to zero in order to force the gluon propagator to only depend on $Q^{2}$, as is the case in the perturbative domain. The assumed vanishing of $B\left(Q^{2}\right)$ leads to a $1 / Q^{4}$ behavior of the gluon propagator. Since there are no ghosts in this gauge, one predicts a singular behavior $\alpha_{s} \propto 1 / Q^{2}$ 
in the IR. This behavior seems satisfactory, given the expectation that it leads to a linear Q- $\bar{Q}$ potential; see Section 4.3. However, more complete SDE calculations in covariant gauges and subsequent lattice calculations have shown this behavior to be an artifact of the assumption $B\left(Q^{2}\right)=0$.

Another advantage of the Landau gauge is that the equations involving the transverse and longitudinal propagators decouple. The on-shell gluon propagator should be transverse, and thus it can be studied independently. In addition, in the Landau gauge the ghost-gluon vertex has a finite renormalization constant [32, 230], which allows the coupling to be defined and extracted from Eq. (4.25); using calculations involving only the two-point Green's functions $G\left(Q^{2}\right)$ and $Z\left(Q^{2}\right)$. The Landau gauge is also symmetric under the exchange of ghost and anti-ghost. The gauge-dependence of the running coupling using this procedure has been argued to be weak for couplings computed in gauges close to the Landau gauge. This was checked by using various linear covariant gauges [223]. Notwithstanding the preeminence of the Landau gauge, other gauges, such as the Coulomb, Feynman [231, 232, 233], axial [229], light-cone [226], maximal Abelian gauge, [234] and linear covariant gauges (which interpolate between the Landau and the Feynman gauges) have also been used [223, 235, 236].

\subsubsection{Classes of solutions in the IR domain}

There is presently a consensus that two types of solutions are possible for the IR behavior of the gluon propagator, and thus for the IR behavior of $\alpha_{s}$. The first solution, called "scaling scenario" (or also "conformal scenario", "critical solution" or "ghost dominance scenario"), was first obtained and studied using the SDE formalism. Many results obtained using the SDE (see e.g. [237]-[245]), Lattice (see Section 4.5), variational approach to the Yang-Mills Schrödinger equation of the vacuum [235] and the exact renormalization group equations method (Section 4.6), show that in the IR, $G\left(Q^{2}, \mu^{2}\right)$ and $Z\left(Q^{2}, \mu^{2}\right)$ scale with related power laws: $G\left(Q^{2}, \mu^{2}\right) \propto\left(Q^{2}\right)^{-\kappa^{\prime}}$ and $Z\left(Q^{2}, \mu^{2}\right) \propto$ $\left(Q^{2}\right)^{2 \kappa^{\prime}}$, where $\kappa^{\prime}$ is called the $I R$ exponent. (This coefficient is generally labeled $\kappa$, or sometimes $\alpha$, in the literature. We write it here $\kappa^{\prime}$ to differentiate it from the mass scale $\kappa$ used in holographic QCD in Sections 3.9.6 and 4.2.). Such relations imply an IR freezing of $\alpha_{s}^{g h}$, see Eq. (4.25); thus the alternative name of "conformal scenario". These relations also imply that ghosts dominate in the IR over gluons. It was shown that the scaling scenario in the Landau gauge leads to a well defined running of the coupling [241, 246, 247, 248]. In this gauge, the $I R$ exponent was calculated to be $\kappa^{\prime}=$ $(93-\sqrt{1201}) / 98 \simeq 0.595$, using the SDE $[235,237,241]$ or the functional renormalization 
group method $[249,250]$. This value leads to an IR freezing value $\alpha_{s}^{g h}(0) \simeq 2.972$.

It should be noted that the SDE computations of $\kappa^{\prime}$ can depend on the approximations used to solve the SDE. This has resulted in a range of values of $0.3 \lesssim \kappa^{\prime} \lesssim 1$. Lattice calculations indicate that $0.5 \lesssim \kappa^{\prime} \lesssim 0.6$ with $\kappa^{\prime} \simeq 0.5$ favored, see e.g. [251]. Lerche and von Smekal [241], and then Alkofer and collaborators [252] have studied $\alpha_{s}^{g h}$ within this range using the Landau gauge and have obtained $2.5 \lesssim \alpha_{s}^{g h}(0) \lesssim 2.972$. The scaling solution also appears in other gauges. It has been studied in the Coulomb gauge by Burgio et al. [253] using the lattice, and by Epple et al. [254] using the SDE. The IR exponent $\kappa^{\prime}$ is computed in the Landau, interpolating and Coulomb gauges in Ref. [235], confirming $\kappa^{\prime} \simeq 0.595$ in the Landau and interpolating gauges. In the Coulomb gauge, two possible values are found: $\kappa^{\prime} \simeq 0.398$ and $\kappa^{\prime}=0.5$. These values are also found using the "functional renormalization group" framework [248] - see Section 4.6 and by using a variational approach in Refs. [210, 254]. However, a solution with $\kappa^{\prime}=0.5$ seems not to be favored [248].

Fisher and collaborators have studied the effects of a finite volume in lattice simulations and the corresponding effects of boundary conditions on the value of $\kappa^{\prime}$ [255] by performing SDE calculations both on a torus and for infinite space; one finds $\kappa^{\prime} \simeq 0.5$ and $\kappa^{\prime} \simeq 0.6$, respectively. The coupling $\alpha_{s}^{g h}$ freezes in the IR, irrespective of the value of $\kappa^{\prime}$. The work of Fisher et al. also indicates an IR-vanishing gluon propagator in the case of infinite space, whereas it stays finite in the finite-volume case. The dependence of $\kappa^{\prime}$ with variable spatial dimensions was studied in [256] for 2, 3 and 4 dimensions. The exponent $\kappa^{\prime}$ was found to not vary dramatically, suggesting similar behavior of the underlying Green's functions in these spaces.

A second possible solution for the IR behavior of $\alpha_{s}$, called the "decoupling solution" (or "massive solution", or "subcritical solution"), was discovered by Boucaud and collaborators using lattice calculations [257]. In this case, the ghost propagator dressing function $G\left(Q^{2}, \mu^{2}\right)$ is constant in the IR and the gluon propagator dressing function scales as $Z\left(Q^{2}, \mu^{2}\right) \propto Q^{2}$. The predicted $I R$ exponents are different: 0 for the ghosts and 1 for the gluons. Thus according to Eq. (4.25) this solution produces an IR-vanishing $\alpha_{s}^{g h} \propto Q^{2}$. The name decoupling solution originates from the vanishing of $Z\left(Q^{2}, \mu^{2}\right)$ as $Q^{2} \rightarrow 0$, leading to free ghosts in the IR limit. That is, the ghosts decouple from an effectively massive longitudinal gluon field. In the decoupling scenario, the IR regime is still dominated by ghosts, although less strongly than in the scaling scenario where they diverge.

The suppression of the gluon field in the decoupling solution can be interpreted as due 
to a dynamically generated gluon mass. The decoupling solution is thereby consistent with a Yukawa-like gluon propagator in the IR. The massive solution is thus sometimes associated with massive scenarios, such as Cornwall's [226, 258] massive gluon which will be discussed in the next section. However, Cornwall's pioneering work is not in the Landau gauge; it does not require ghosts, and it uses a different coupling, $\alpha_{g s e}$ (although it has been shown to be similar to $\alpha_{s}^{g h}[227]$ ). Cornwall's results produce a non-vanishing freezing $\alpha_{g s e}$.

Although it was first found on the lattice, the decoupling solution is also present in the SDE calculations [247, 257, 259, 260, 261], the pinch technique background field method [262], an extended Gribov-Zwanziger approach [263, 264], and from the stochastic quantization equations [266]. This last work suggests, within the approximations used to truncate the equations, that the decoupling solution is a general solution of the theory. In contrast, the scaling solution would appear in specific gauges only, and represent a particular solution stemming from the existence of a Gribov horizon (i.e., the limit of the finite region of the field configuration space where the ghost propagator remains positive and finite, see Section 4.7). This work also indicates that the decoupling solution has less effective action, which explains why it seems favored by the lattice technique.

It is still unclear which solution is realized in the physical world. Certainly, the description given above of the two solutions is gauge-dependent, since some gauges, some relativistic form of dynamics, e.g., the light-front, and some quantization methods do not require ghosts. In such a case, the ghosts are replaced by other degrees of freedom, such as the longitudinal component of the gluon propagator, see Section 4.8. Thus the two solutions can still exist in a gauge without ghosts.

Some authors [255] have indicated that some decoupling-like results (that is calculations leading to a vanishing coupling) may be due to a finite lattice-size effect. However, an unphysical decoupling originating solely from finite size effects seems to be now ruled out [267], and it would not explain why the solution is also present in analytical methods. Fischer, Maas and Pawlowski have argued that only the scaling solution is compatible both with the equations respecting the BRST symmetry [268], and with the color-confining gluons [261]. Fischer and Pawlowski have also showed that the scaling solution is unique for the SDE and the exact renormalization group equations. In particular, they showed that if the ghost and gluon external momenta vanish, then one must realize the scaling solution [246]. The study of Alkofer, Huber and Schwenzer also disfavors the decoupling solution, at least in the simplest realizations of QCD's confinement [247]. Furthermore, the decoupling solution cannot satisfy the Kugo-Ojima confinement 
criterion [269]. On the other hand, there are models supporting the decoupling solution: Boucaud et al. have interpreted this behavior in the context of instantons [165]: the initial $\alpha_{s}\left(Q^{2}\right) \propto Q^{4}$ IR-behavior is predicted by the instanton liquid model, which represents the QCD vacuum as an instanton liquid.

It has been shown in [217] and discussed in [143] that the two solutions lead to sensi-

bly similar descriptions of Nature in the IR domain, corresponding to hadron mass/size scales. However, it is important to determine which solution is being realized; for example, the scaling solution justifies the approach based on conformal field theories, see Sections 3.9.6 and 4.2. In this regard, the excellent description of QCD phenomenology by this approach and the physical arguments for IR freezing of the QCD coupling given in Refs. [5, 6], would suggest that the scaling solution is the one realized in Nature.

\section{Typical forms of the gluon propagator for the two solutions}

The scaling solution, which leads to a freezing infrared fixed point value for the QCD coupling, is consistent with the original Gribov-Zwanziger approach of confinement (Section 4.7). In this case the gluon propagator behaves as

$$
D_{\mu \nu}^{b c}(q)=\delta^{b c}\left(\eta_{\mu \nu}-\frac{q_{\mu} q_{\nu}}{q^{2}}\right) \frac{q^{2}}{q^{4}+m_{g r}^{4}} .
$$

Here, $m_{g r}$ is the Gribov mass. In addition, if $Q^{2} \gg m_{g r}^{2}$ one recovers the perturbative gluon propagator $\delta^{b c}\left(\eta_{\mu \nu}-q_{\mu} q_{\nu} / q^{2}\right) / q^{2}$.

The decoupling solution, which leads to an IR-vanishing coupling when $Q^{2} \rightarrow 0$, is itself consistent with an effective gluon propagator obeying a Yukawa form [270],

$$
D_{\mu \nu}^{b c}(q)_{q \rightarrow 0} \propto \delta^{b c}\left(\eta_{\mu \nu}-\frac{q_{\mu} q_{\nu}}{q^{2}}\right) \frac{1}{q^{2}-m_{g}^{2}},
$$

which can also be consistent with the generalized Gribov-Zwanziger scenario. Nevertheless, in this refined Gribov-Zwanziger scenario, the scaling solution still seems preferred $[271]$.

We will now discuss the topic of the effective gluon mass. As we have mentioned earlier, the emergence of such a mass is equivalent to the regularization of the behavior of $\alpha_{s}$ in the IR. 


\subsubsection{The massive gluon propagator}

The first studies of the gluon propagator in the IR regime using the SchwingerDyson formalism began in the 1970s [258, 272, 273]. Using the Landau gauge, these early results indicated that $\alpha_{s}$ has no Landau pole, and that in the $\operatorname{IR}, \alpha_{s}\left(Q^{2}\right) \propto 1 / Q^{2}$ $[272,274]$. A calculation in the axial gauge [229] reached the same conclusion. The singular dependence in the IR agrees naively with the expectation of a linear potential in the static limit; see Section 4.3. However, in spite of this apparent consistency, Cornwall [226] has argued that the $1 / Q^{2}$ behavior is actually erroneous due to the explicit exclusion of solutions which generate a dynamical gluon mass that regulates the Landau pole. Earlier suggestions that a gluon mass should suppress the unphysical Landau pole were made in $[258,275,276]$.

In Cornwall's work an analytic form for the gluon propagator is proposed for solving the SDE in the pure gauge sector and in the light-cone gauge where ghosts are unnecessary. Feynman graphs are resummed to form a gauge-independent gluon propagator dressing function that modifies the free gluon propagator which carries the gauge dependence. The resulting dynamically generated gluon effective mass is $Q^{2}$-dependent and vanishes at large- $Q^{2}$. This feature ensures compatibility with pQCD expectations, and it accommodates the fact that a physical gluon mass is forbidden by gauge invariance. Cornwall's SDE solution to the gluon propagator suggests that the perturbative form of $\alpha_{s}$, defined from the gluon self-energy, should be modified in the IR as :

$$
\alpha_{g s e}\left(Q^{2}\right)=\frac{4 \pi}{\beta_{0} \ln \left(\left[Q^{2}+\varsigma m_{g}^{2}\left(Q^{2}\right)\right] / \Lambda^{2}\right)},
$$

with $\varsigma=4$ and $m_{g}\left(Q^{2}\right)$ is an effective gluon mass:

$$
m_{g}^{2}\left(Q^{2}\right)=\frac{m^{2}}{\left[\ln \left(\frac{Q^{2}+\xi m^{2}}{\Lambda^{2}}\right) / \ln \left(\frac{\varsigma m^{2}}{\Lambda^{2}}\right)\right]^{12 / 11}},
$$

with $m=0.5 \pm 0.2 \mathrm{GeV}$ and $\Lambda=0.26 \pm 0.05 \mathrm{GeV}$. The freezing value, $\alpha_{\text {gse }}(0)=$ $0.42 \pm 0.20$ agrees with the characteristic value of $\alpha_{s}$ estimated using magnetic and color-magnetic spin-spin interactions [277] and other determinations done in the context of hadron spectroscopy, see Section 4.3. The limit $m=\Lambda$ is consistent with Richardson's form, Eq. (4.19). Renormalization group equations predict that at large $Q^{2}, m_{g}^{2}\left(Q^{2}\right) \propto$ $\ln \left(Q^{2}\right)^{-12 / 11}$ where the exponent is related to the strength of the static force in the pure gauge sector; $4 \pi\left(\beta_{0}\right)^{-1}=12 \pi / 11$. 
The gluon mass must satisfy the condition $m_{g} \geq \Lambda$ in order to eliminate the Landau pole. Cornwall showed that using the pinch technique $m \simeq 1.2 \Lambda$ [228] (A review of the pinch technique, including its application to the SDE and the computation of $\alpha_{s}$, can be found in [278]). Values of $m$ and $\Lambda$ can also be obtained by assuming Cornwall's massive form for the gluon propagator and constraining it with hadron phenomenology. For example, the isovector GDH sum rule for the nucleon can be used to constrain $m_{g}$ by determining $\alpha_{\overline{M S}}$ at $Q^{2}=0$. Solving Eqs. (4.1) and (4.4), one finds $m=0.350 \mathrm{GeV}$ and $\Lambda=0.268 \mathrm{GeV}\left(\alpha_{g s e}(0)=0.59\right)$. The ratio $m / \Lambda=1.31$ validates the pinch technique expectation that $m / \Lambda \simeq 1.2$ [228]. The GDH-based result is gauge independent, but this analysis depends on the truncation order of Eq. (3.40). Since an asymptotic series will reach its optimal order at $n \sim(1 /$ coupling $)$, the value of $\alpha_{s}\left(Q^{2} \simeq 1 \mathrm{GeV}^{2}\right)$ suggests that truncating Eq. (3.40) at $n \sim 4$ is adequate.

Another way to use phenomenological information is to constrain Eqs. (4.28) and (4.29) using data for hadron form factors. A good description of the experimental pion and kaon form factors is obtained for $m=0.54 \mathrm{GeV}$ by Ji and collaborators [279]. It yields $\alpha_{g s e}(0)=0.4$ for $\Lambda=0.26 \mathrm{GeV}$. Brodsky and collaborators have combined the dimensional counting rules for the form factors in the UV with Cornwall's freezing coupling for $m=0.44 \mathrm{GeV}$ (i.e. $\alpha_{g s e}(0)=0.47$ for $\Lambda=0.26 \mathrm{GeV}$ ) to analyze the photon-to-pion transition form factor and $\gamma \gamma \rightarrow \pi^{+} \pi^{-}$. The data is well reproduced, but the predicted normalization of the space-like pion form factor is higher than measurements [280]. On the other hand, Aguilar and collaborators find that $m=0.300 \mathrm{GeV}$ [281] describes the pion form factor data well, which implies a freezing value $\alpha_{\text {gse }}(0)=0.68(\Lambda=0.26 \mathrm{GeV})$.

In Ref. [282], the value of $m$ is constrained using measurements of the proton-proton elastic cross section. This yields $m=0.370 \mathrm{GeV}\left(\alpha_{g s e}(0)=0.55\right.$ for $\left.\Lambda=0.26 \mathrm{GeV}\right)$ and in Refs. $[283,284]$ the value $m=0.374 \mathrm{GeV}\left(\alpha_{\text {gse }}(0)=0.54\right.$ for $\left.\Lambda=0.26 \mathrm{GeV}\right)$ is obtained. The SDE are used in Ref. [285] to obtain $m=0.68 \mathrm{GeV}\left(\alpha_{g s e}(0)=0.35\right.$ for $\Lambda=0.26 \mathrm{GeV})$. In this analysis, the predicted SDE coupling freezes at $\alpha_{s}(0)=2.8$, a much larger value than the $\alpha_{g s e}(0)$ expected from Cornwall's form. In Ref. [285], the gluon mass and coupling are well parameterized in the UV and IR-UV transition region $1<Q^{2}<18.5 \mathrm{GeV}^{2}$ by

$$
m_{g}^{2}\left(Q^{2}\right)=\frac{0.22+0.019 Q^{2}}{1+1.76 Q^{2}}
$$

and

$$
\alpha_{s}\left(Q^{2}\right)=12.9 m_{g}^{2}\left(Q^{2}\right) .
$$

All of these values of $m$ are within Cornwall's proposed range. Lattice QCD estimates 
of $m$ also fall within this range [286]. An analysis of the data for inclusive radiative decays of the $J / \psi$ and $\Upsilon$ yields a somewhat higher value, $m \sim 1 \mathrm{GeV}\left(\alpha_{\text {gse }}(0) \sim 0.28\right.$ for $\Lambda=0.26 \mathrm{GeV}$ ) [287].

Aguilar and Papavassiliou have summarized the studies of $m_{g}\left(Q^{2}\right)$ in Ref. [232]. These authors also show that a massive propagator effectively describes the solution of the SDE in the gauge-independent pinch technique in the pure gauge sector, but with two possible behaviors at large $Q^{2}$. One solution is similar to Eq. (4.29), with $m_{g}\left(Q^{2}\right) \propto \ln \left(Q^{2}\right)^{-\gamma}$ (taking a typical value $\gamma=0.86$ ), as expected from renormalization group equations. The second solution falls with a power law: $m_{g}\left(Q^{2}\right) \propto \ln \left(Q^{2}\right)^{\gamma} / Q^{2}$ (with a typical value $\gamma=1.12$ ), similarly to the power corrections obtained from the $O P E$. The behavior of the gluon mass in Eq. (4.29) is consistent with the logarithmic running of $m_{g}\left(Q^{2}\right)$, as predicted by the first solution, with the values $\varsigma=1.007, m=0.3$ $\mathrm{GeV}, \Lambda=0.3 \mathrm{GeV}$. This leads to a freezing value for $\alpha_{\text {gse }}(0) \simeq 1.2$. For the second power-law solution for $m_{g}\left(Q^{2}\right)$, the effective mass is well described by

$$
m_{g}^{2}\left(Q^{2}\right)=\frac{m^{4}}{\left[Q^{2}+m^{2}\right]\left[\ln \left(\frac{Q^{2}+\epsilon m^{2}}{\Lambda^{2}}\right) / \ln \left(\frac{\epsilon m^{2}}{\Lambda^{2}}\right)\right]^{\gamma}},
$$

with $\epsilon=1.046, m=0.5 \mathrm{GeV}, \Lambda=0.3 \mathrm{GeV}$ and $\gamma=1.12$. This leads to the freezing value $\alpha_{\text {gse }}(0) \simeq 1.0$.

The results of Aguilar and Papavassiliou are gauge-invariant, and the effective gluon mass can be interpreted as a gauge-invariant gluon vacuum condensate or nonperturbative correction of dimension four. In subsequent work, [233], Aguilar and Papavassiliou used the Feynman gauge, the pinch technique, and gauge invariant truncations of the gluon propagator to obtain forms for $m_{g}\left(Q^{2}\right)$ and $\alpha_{g s e}\left(Q^{2}\right)$. Effects from ghost loops were neglected. Although the authors did not specify the freezing value, they provide a numerical example with a freezing value of $\alpha_{g s e}(0)=0.91$ and a gluon mass normalization, $m_{g}(0)=0.45 \mathrm{GeV}$. An alternative nonperturbative approach to the gluon mass has been developed by Aguilar and collaborators based on the SDE in the Landau gauge [283] and the RGE [284]. The authors provide a numerical fit of their results:

$$
m_{g}^{2}\left(Q^{2}\right)=\frac{m^{2}}{1+\left(Q^{2} / M^{2}\right)^{\gamma}}
$$

with $M=0.557 \mathrm{GeV}, m=0.375 \mathrm{GeV}$, and $\gamma=1.08$. Cornwall's solution (4.29), agrees well with these results. The gauge-dependence of $m_{g}\left(Q^{2}\right)$ in these formulae has been 
investigated using linear covariant gauges in which a gauge parameter $\xi$ continuously transitions between the Landau gauge $(\xi=0)$ and the Feynman gauge $(\xi=1)$. The resulting gluon mass normalization is typically $m \simeq 0.37 \pm 0.1 \mathrm{GeV}$ [236]. This implies $\alpha_{\text {gse }}(0)=0.55 \pm 0.03$ for $\Lambda=0.26 \mathrm{GeV}$.

A closely related model has been recently proposed by Ayala et al. [288]. In this analysis the effective charge $\alpha_{V}$ has the form proposed by Cornwall, Eq. (4.28), assuming the Aguilar et al. $Q^{2}$-dependence of the effective gluon mass, Eq. (4.33), with $\gamma=1.15$ and a normalization $m=\Lambda$. Thus Richardson's limit, Eq. (4.19), of Cornwall's coupling is recovered at $Q^{2}=0$. Since the resulting coupling diverges as $1 / Q^{2}$ when $Q^{2} \rightarrow 0$, this also leads to a linear potential for static quarks consistent with quarkonium spectroscopy.

Cornwall's coupling, Eq. (4.28), takes the form of the pQCD coupling at $\beta_{0}$ order with an additional dynamical effective gluon mass term. In effect, the nonperturbative effects which regularize $\alpha_{s}$ in the IR are effectively incorporated into $m_{g}\left(Q^{2}\right)$, together with higher order pQCD loops. This is similar to the case of the effective charges defined by Grunberg, see Section 4.1: both nonperturbative and perturbative contributions are incorporated into $\alpha_{s}\left(Q^{2}\right)$. However, in Cornwall's case, observable-independent higher loops are folded in, whereas in Grunberg's case, observable-dependent gluonic bremsstrahlung is folded in. Nevertheless, the two results can agree, once the scheme dependence is accounted for, see Section 5.

Eqs. (4.28) and (4.29) have been augmented by further work by Cornwall and Papavassiliou [289] using the pinch technique and the SDE. One finds the same form for the running coupling except that quark loops are now included:

$$
\alpha_{\text {gse }}\left(Q^{2}\right)=\frac{12 \pi}{33 \ln \left[\left(Q^{2}+\epsilon m_{g}^{2}\left(Q^{2}\right)\right) / \Lambda^{2}\right]-2 n_{f} \ln \left[\left(Q^{2}+\epsilon M^{2}\right) / \Lambda^{2}\right]} \text {. }
$$

In this analysis, $\epsilon \simeq 4.8, M$ is identified with the string tension: $M \simeq \sqrt{\sigma} \simeq 0.42$ $\mathrm{GeV}$, and Eq. (4.29) is used for $m_{g}\left(Q^{2}\right)$. The screening role of the quark loops can be recognized in Eq. (4.34). With $n_{f}=2, \Lambda=0.26 \mathrm{GeV}$ and $M=0.42, \alpha_{g s e}(0)=0.44$.

Other forms for the massive gluon propagator have been proposed using the GribovZwanziger approach; see Eq. (4.26). If one uses the SDE solution of Stingl and of Habel et al. [290], the gluon propagator has the form

$$
D_{\mu \nu}^{b c}(q)=\delta^{b c}\left(\eta_{\mu \nu}-\frac{q_{\mu} q_{\nu}}{q^{2}}\right) \frac{q^{2}}{q^{4}+2 a q^{2}+m_{g}^{4}},
$$


where $a$ is a parameter constrained by phenomenology.

Lattice results can also be interpreted in terms of a massive gluon propagator [291]. For example, the results of Marenzoni and collaborators [292] can be fit by the form

$$
D\left(Q^{2}\right)=\frac{1}{b Q^{2}\left(Q^{2} / \Lambda^{2}\right)^{\eta}+m_{g}^{2}},
$$

with $b=0.102(1), \eta=0.532(12)$ and $m_{g} \simeq 0.4 \mathrm{GeV}$. Similarly, Oliveira and Bicudo have interpreted their lattice data in term of a massive gluon propagator. Their result for

$Q^{2} \lesssim 0.25 \mathrm{GeV}^{2}$ can be described assuming a constant IR gluon mass of about $m_{g} \simeq 0.7$ $\mathrm{GeV}$ [293]. Their calculations are performed in the Landau gauge. A similar value is obtained in Ref. [263]. The results are consistent with the decoupling solution, yielding an IR-vanishing $\alpha_{s}$. In recent work [285], the SDE and lattice data are also combined in the form of a massive gluon propagator. This leads to an IR value of $\alpha_{s}(0) \simeq 0.9 \pi$. Thus lattice studies can in effect be interpreted as the emergence of an effective gluon mass.

Massive gluons have also been considered in approaches other than SDE and lattice QCD; e.g., the Gribov-Zwanziger approach [294], the background perturbation theory (Section 4.10), or the variational approach of Szczepaniak and Swanson [157] which yields a constituent gluon mass of about $0.6 \mathrm{GeV}$.

Finally, we notice that an effective gluon mass can be used to generate $1 / Q^{2}$ power corrections; this is in spite of the fact that in the OPE there is no condensate of dimension-2 arising from a local gauge-invariant operator [295]. However, in this case, the resulting effective gluon mass must be imaginary (tachyonic gluon mass).

\subsubsection{The coupling defined from the ghost-gluon vertex}

The ghost-gluon coupling $\alpha_{s}^{g h}$ from Eq. (4.25) was first obtained using the SDE formalism by von Smekal, Hauck and Alkofer [238]. Their Landau gauge analysis is based on a self-consistent truncation scheme, where the truncation implements the SlavnovTaylor identities [32] for the three-point vertex function. The numerical results lead to a coupling agreeing with pQCD evolution at large $Q^{2}$, while freezing to a value of 
$\alpha_{s}^{g h}(0) \simeq 9.5$. The following forms match well the numerical solution [281]:

$$
\begin{aligned}
\alpha_{s}^{g h} & =0.261+9.2621 e^{-2 \frac{\left(Q^{2}-0.0297\right)^{2}}{0.4689}} ; \text { for } Q^{2}<0.31 \mathrm{GeV}^{2}, \\
\alpha_{s}^{g h} & =1.4741+8.6072 e^{-\frac{Q^{2}-0.1626}{0.3197}} ; \text { for } 0.31<Q^{2}<1.3 \mathrm{GeV}^{2}, \\
\alpha_{s}^{g h} & =\frac{1.4978}{\ln \left(1.8488 Q^{2}\right)} ; \text { for } Q^{2}>1.3 \mathrm{GeV}^{2} .
\end{aligned}
$$

The numerical techniques have been subsequently improved, yielding smaller freezing values. Fischer et al. [242] obtained a numerical coupling (Landau gauge, pure gauge sector) which is well fitted by:

$$
\alpha_{s}^{g h}(Q)=\frac{2.972}{\ln \left(e+5.292 Q^{2.324}+0.034 Q^{3.169}\right)},
$$

where the numerical coefficients are determined by matching to the value of the pQCD coupling at the $Z^{0}$ mass: $\alpha_{s}^{g h}\left(M_{Z}^{2}\right)=\alpha_{s}^{p Q C D}\left(M_{Z}^{2}\right)$. This implies, in the MOM scheme, $\Lambda_{M O M}=0.715 \mathrm{GeV}$ and the freezing value $\alpha_{s}^{g h}(0) \simeq 2.972$; see Section 4.4.3.

An alternative truncation prescription was used by Bloch [296]. The result in the pure gauge theory is fitted by:

$$
\alpha_{s}^{g h}\left(Q^{2}\right)=\frac{1}{15+Q^{2} / \Lambda^{2}}\left(15 \times 2.6+\frac{4 \pi}{\beta_{0}}\left(\frac{1}{\ln \left(Q^{2} / \Lambda^{2}\right)}-\frac{1}{Q^{2} / \Lambda^{2}-1}\right) \frac{Q^{2}}{\Lambda^{2}}\right),
$$

where $\Lambda_{\overline{M S}}=0.33 \mathrm{GeV}$ in the $\overline{M S}$ scheme. In this Landau gauge analysis, the freezing value for $\alpha_{s}^{g h}$ is 2.6, and the dynamically generated gluon mass is $m_{g} \simeq 0.35$ in the IR domain. The form for the running ghost-gluon coupling is similar to the behavior of $\alpha_{a n}$ obtained in the analytic approach, Eq. (4.54), see Section 4.9.1. The truncation prescription was subsequently improved in Ref. [245], leading to numerical results parameterized as

$$
\begin{aligned}
\alpha_{s}^{g h}\left(Q^{2}\right)=\frac{1}{1.16+Q^{4} / \Lambda^{4}}\left(3.49\left[1.16-0.070\left(Q^{2} / \Lambda^{2}\right)^{0.66}\right]\right. & \\
& \left.+\left[Q^{4} / \Lambda^{4}\right]\left(Q^{2} / \Lambda^{2}+2\right) \alpha_{s}^{p Q C D, \beta_{1}}\right)
\end{aligned}
$$

where $\alpha_{s}^{p Q C D, \beta_{1}}$ is the perturbative coupling at order $\beta_{1}$ obtained from Eq. (3.24) and $\Lambda=0.856 \mathrm{GeV}$. This gives a freezing value of 3.49. Comparisons with the earlier 
results illustrate the effects of modifying the truncation prescription as well as choosing a different RS. To reinforce this point, we note that the earlier work by Atkinson and Bloch with a bare truncation that leads to a freezing value of 11.47 by averaging the SDE angular integrals, and to a freezing at $4 \pi / 3$ with the angular integrals done exactly [239]. Bloch systematically studied the effect of varying the truncation scheme and found that $2 \pi / 3<\alpha_{s}^{g h}(0)<8 \pi / 3$ in $\mathrm{SU}(3)[240]$.

Aguilar and Natale [259] obtained an IR-vanishing coupling $\alpha_{s}^{g h}$. These authors assumed a massless gluon propagator behaving as $1 / Q^{2}$. The calculations were done in the Landau gauge and pure Yang-Mills using the renormalization prescription of [226, 289]. In subsequent works Aguilar et al. employed a massive gluon propagator, rather than a massless one, leading to a freezing behavior of the coupling. Furthermore, in [227], Aguilar et al. computed both $\alpha_{g s e}$ and $\alpha_{s}^{g h}$ and found that the two couplings have the same freezing value of order 0.6 for $m_{g}(0)=0.5 \mathrm{GeV}$.

Schleifenbaum and collaborators [235] have computed the gluon and ghost propagators in both Landau and Coulomb gauges, finding that the gluon propagator in the IR is suppressed, whereas the ghost propagator is singular. These features yield an approximately linear potential and a ghost-gluon coupling freezing at $\alpha_{s}^{g h}(0) \simeq 3.65$ for the Landau gauge. They also find two solutions in the Coulomb gauge $\alpha_{s}^{g h}(0) \simeq 14.21 / N_{C}=4.74$ or $\alpha_{s}^{g h}(0)=16 \pi / N_{C} \simeq 5.59$, with the value 4.74 favored [248].

\subsubsection{Couplings defined from the 3-gluon and 4-gluon vertices}

In addition to the definition from the ghost-gluon vertex, Eq. (4.25), couplings can also be defined from the 3-gluon and 4-gluon vertices:

$$
\begin{aligned}
& \alpha_{s}^{3 g}\left(Q^{2}\right)=\alpha_{s}^{3 g}(\mu)\left[\Gamma^{3 g}\left(Q^{2}, \mu\right)\right]^{2} Z^{3}\left(Q^{2}, \mu\right), \\
& \alpha_{s}^{4 g}\left(Q^{2}\right)=\alpha_{s}^{4 g}(\mu)\left[\Gamma^{4 g}\left(Q^{2}, \mu\right)\right]^{2} Z^{2}\left(Q^{2}, \mu\right),
\end{aligned}
$$

where $\Gamma^{3 g}$ and $\Gamma^{4 g}$ are the dressing functions of the tree-level 3-gluon and 4-gluon vertices. In contrast to the case of $\alpha_{s}^{g h}$, dressing functions appear since there are no SlavnovTaylor identities for these vertices. The three couplings $\alpha_{s}^{g h}\left(Q^{2}\right), \alpha_{s}^{3 g}\left(Q^{2}\right)$ and $\alpha_{s}^{4 g}\left(Q^{2}\right)$ are different in the MOM RS but can be related to each other $[22,145]$.

The 3-gluon and 4-gluon vertices have been studied in the pure gauge sector by Baker and Lee [297], Celmaster and Gonsalves [22], by Brandt and Frenkel [298], as well as by Papavassiliou using the pinch technique [299]. The resulting couplings $\alpha_{s}^{3 g}\left(Q^{2}\right)$ 
and $\alpha_{s}^{4 g}\left(Q^{2}\right)$ were first investigated by Alkofer, Fischer, and Llanes-Estrada [300]. They concluded that these couplings must freeze in the IR, but they did not provide freezing values. A recent perturbative analytical calculation of the IR and UV behaviors of the 3-gluon vertex (Landau gauge and pure gauge sector) is reported in [301].

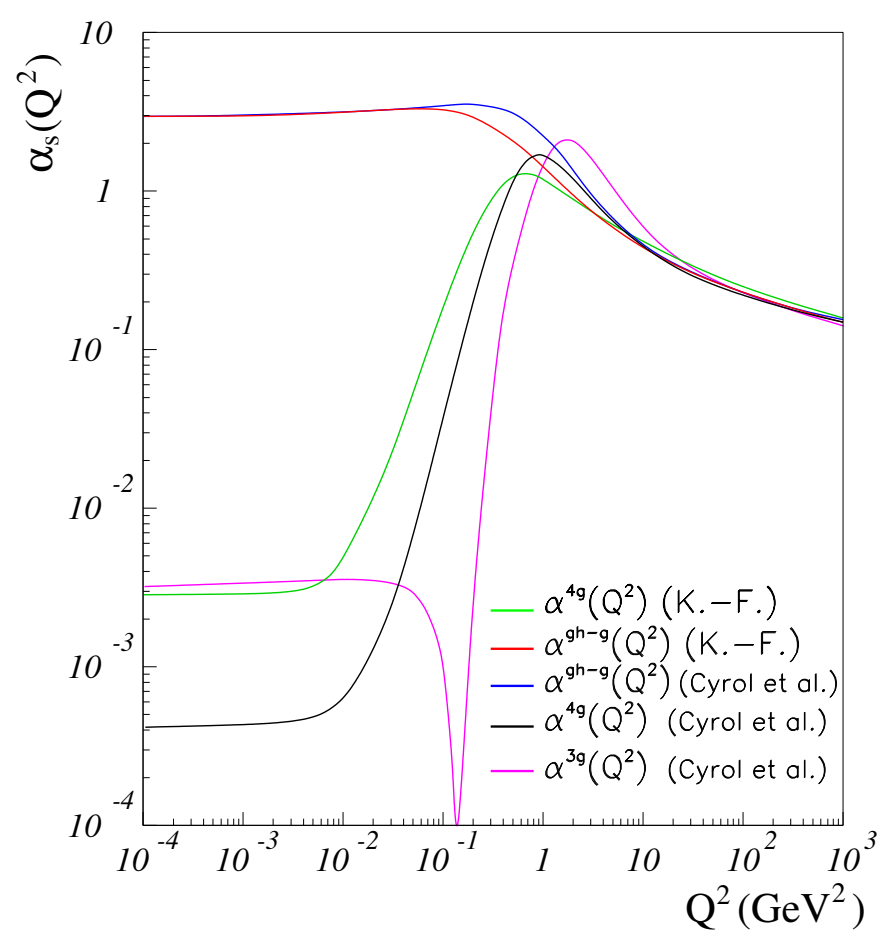

Figure 4.4: (Color online) SDE computations of $\alpha_{s}$ defined using the 4-gluon vertex (the green line is from Ref. [306] and black line is from Ref. [307]). The red line, using the ghost-gluon vertex definition, is from Ref. [306] and the blue line is from Ref. [307]. The magenta line for the 3-gluon vertex definition is from Ref. [307]. Quark degrees of freedom are not included and the calculations are done in the Landau gauge and MOM scheme.

The 3-gluon vertex was calculated nonperturbatively using the SDE by Eichmann et al. [302] using the Landau gauge. Both scaling and decoupling solutions were obtained. In the decoupling case, $\alpha_{s}^{g h}$ and $\alpha_{s}^{3 g}$ vanish in the IR, whereas in the scaling case $\alpha_{s}^{g h}(0)=$ 2.972 and $\alpha_{s}^{3 g}(0)=1.6 \times 10^{-3}$; see Fig. 4.4. A self-consistent SDE calculation of $\alpha_{s}^{3 g}\left(Q^{2}\right)$, also in the Landau gauge, yields vanishing $\alpha_{s}^{g h}\left(Q^{2}\right)$ and $\alpha_{s}^{3 g}\left(Q^{2}\right)$ in the IR [303]. Huber, Campagnari, and Reinhardt solved numerically, in the Coulomb gauge and pure gauge sector, the canonical recursive SDE for the three-gluon and ghost-gluon vertices. The resulting ghost-gluon vertex is IR finite, whereas the three-gluon vertex is IR singular [304].

The 4-gluon vertex was first studied nonperturbatively by Stingl, Driesen and col- 
laborators [305]. The calculation for the 4-gluon coupling $\alpha_{s}^{4 g}$ in the pure gauge sector, assuming Landau gauge and the MOM RS, by Kellermann and Fischer [306] yields a very small freezing value, $\alpha_{s}^{4 g}(0) \simeq 2.77 \times 10^{-3}$. The resulting behavior of $\alpha_{s}^{4 g}\left(Q^{2}\right)$ follows the pQCD expectation at large $Q^{2}$ and increases with decreasing $Q^{2}$ to about $Q^{2}=0.5$ $\mathrm{GeV}^{2}$. It then drops to its freezing value, which is reached at $Q^{2} \sim 10^{-2} \mathrm{GeV}^{2}$; see Fig. 4.4.

Cyrol, Huber and von Smekal [307] have recently computed self-consistent values for the couplings controlling the 3-gluon and 4-gluon vertices. Again this calculation is in the pure gauge sector, assumes Landau gauge and the MOM RS. In the scaling solution, the 3-gluon and 4-gluon vertex couplings freeze at values that are very small compared to $\alpha_{s}^{g h}(0)=2.972 ; \alpha_{s}^{3 g}(0)=3.2 \times 10^{-3}$ and $\alpha_{s}^{4 g}(0)=4.2 \times 10^{-4}$, confirming qualitatively the finding in Refs. [302, 306], namely $\alpha_{s}^{3,4 g}(0) \ll \alpha_{s}^{g h}(0)$. These running couplings are shown in Fig. 4.4. In the decoupling solution case, all three definitions of $\alpha_{s}$ lead to IR-vanishing couplings.

\subsubsection{Including quarks}

Quark loops contribute to the running of $\alpha_{s}$ in the UV in the opposite direction to the gluons, making $\alpha_{s}$ increase with $Q^{2}$. Thus one expects that their inclusion will be to decrease $\alpha_{s}(0)$ compared to the results obtained in the pure gauge sector. This comparison is done with $\Lambda$ adjusted so that $\alpha_{s}^{n_{f}=0}\left(M_{Z}^{2}\right)=\alpha_{s}^{n_{f}=5}\left(M_{Z}^{2}\right)$. A contrario, if the value of $\Lambda$ is kept the same for any $n_{f}$, the quark loops would increase the value of $\alpha_{s}^{n_{f}>0}(0)$ compared to $\alpha_{s}^{n_{f}=0}(0)$ since $\alpha_{p Q C D}^{n_{f}>0}\left(Q^{2}\right)>\alpha_{p Q C D}^{n_{f}=0}\left(Q^{2}\right)$ and the Landau pole divergence of the perturbative coupling is steeper. In any case, since effects other than vacuum polarization are included in the IR definition of the coupling, the effect of quarks is not obvious, as illustrated by the following example.

The influence of quark loops on the coupling has been investigated in ref. [289]; see Eq. (4.34). The quark loops increase the freezing value: $\alpha_{g s e}^{n_{f}=2}(0)=0.81$ in comparison with $\alpha_{g s e}^{n_{f}=0}(0)=0.60$. The values $\Lambda^{(5)}=0.09 \mathrm{GeV}$ and $m=1.2 \Lambda^{(5)}=0.11 \mathrm{GeV}$ are used for the $n_{f}=2$ calculation, and $\Lambda^{(0)}=0.65 \mathrm{GeV}$ and $m=1.2 \Lambda^{(0)}=0.78 \mathrm{GeV}$ are used for the $n_{f}=0$ calculation -so that $\alpha_{g s e}^{n_{f}=2}\left(M_{Z}^{2}\right)=\alpha_{g s e}^{n_{f}=0}\left(M_{Z}^{2}\right)=0.119$ at the $Z^{0}$ pole. The quark loop effect is thus non-negligible according to [289]. However, Fisher and collaborators have recently reported that quark loops are suppressed in the IR and thus have only a small effect for a physical numbers of quark flavors, $n_{f}<6$ [308]. This result is apparent from the function that fits their results (apart for a bump at $Q^{2} \simeq 0.5$ 
$\mathrm{GeV}^{2}$, which is believed to be unphysical and is omitted):

$$
\alpha_{s}^{g h}\left(Q^{2}\right)=\frac{1}{1+\frac{Q^{2}}{\Lambda^{n_{f}}}}\left(2.972+\frac{4 \pi}{\beta_{0}}\left(\frac{1}{\ln \left(\frac{Q^{2}}{\Lambda^{n} f^{2}}\right)}-\frac{1}{\frac{Q^{2}}{\Lambda^{n} f^{2}}-1}\right) \frac{Q^{2}}{\Lambda^{n_{f} 2}}\right)
$$

with a freezing value which is independent of $n_{f}$. The quark effects are encoded in the scale parameter: $\Lambda_{M O M}^{n_{f}=0}=0.71 \mathrm{GeV}$ and $\Lambda_{M O M}^{n_{f}=3}=0.51 \mathrm{GeV}$. (We note that the fit form is similar to Eq. (4.39) and to the coupling found in the analytical approach, Eq. (4.54)). Eq. (4.43) was used to study analytic properties of the gluon and quark propagators in the Landau gauge [244].

Related to the inclusion of quarks, Alkofer, Fisher, and Llanes-Estrada have studied the influence of chiral-symmetry breaking on the running coupling $\alpha_{s}^{q g}\left(Q^{2}\right)$, now defined by the quark-gluon vertex [309]:

$$
\alpha_{s}^{q g}\left(Q^{2}\right)=\alpha_{s}^{q g}(\mu) \xi_{1}^{2}\left(\mu, Q^{2}\right) P^{2}\left(\mu, Q^{2}\right) Z\left(\mu, Q^{2}\right)
$$

where $\xi_{1}$ is the vertex dressing function and $P$ the quark propagator dressing function. It was found that the reduction of the number of linearly independent Dirac tensors using chiral symmetry leads to different behavior of the coupling. In the chiral symmetry case, which approximates well the light quark sector, the coupling is IR-finite: $\alpha_{s}^{q g}\left(Q^{2}\right) \simeq 2.5$. In contrast, in the broken chiral symmetric case (as for the heavy quark sector where quark loops are quenched), the additional tensors induce a coupling diverging in the IR: $\alpha_{s}^{q g}\left(Q^{2}\right) \propto 1 / Q^{2}$. As discussed in Section 4.3 the $1 / Q^{2}$ behavior of the coupling gives rise to a linear $\mathrm{Q}-\overline{\mathrm{Q}}$ potential for heavy static quarks. This behavior can be compared to the expectation that QCD strings develop only in the heavy-quark sector [310].

\subsubsection{Gauge dependence}

Many calculations in the SDE formalism and other frameworks yield a gauge-dependent coupling $\alpha_{s}$. The question of the best choice of gauge and its influence on the IR-behavior of $\alpha_{s}$ is open. (The gauge-dependence subsides in the UV regime). Fisher has reported that couplings computed in gauges close to the Landau gauge have a weak gauge-dependence [223]. Fischer and Zwanziger [311] have studied the gauge-dependence of the scaling solution of $\alpha_{s}^{g h}$ using a class of transverse gauges which interpolate between the Landau and Coulomb gauges. The IR value $\alpha_{s}^{g h}(0) \simeq 2.972$, found for the $I R$ exponent $\kappa^{\prime}=0.595$, holds across the gauge range, except the Coulomb case which has 
a singular limit. This particular case is studied in detail in Ref. [312]. The freezing value is found to be slightly lower: $\alpha_{s}^{g h}(0) \simeq 2.333$. However, the already mentioned calculations of the same quantity in Ref. [235], in both Landau and Coulomb gauges, lead to the opposite trend: $\alpha_{s}^{g h}(0) \simeq 3.65$ in the Landau gauge and $\alpha_{s}^{g h}(0) \simeq 4.74$ in the Coulomb gauge. (Two solutions are actually found in the Coulomb gauge: $\alpha_{s}^{g h}(0) \simeq 4.74$ or $\alpha_{s}^{g h}(0) \simeq 5.59$ for $\kappa^{\prime} \simeq 0.398$ or $\kappa^{\prime}=0.5$, respectively, with $\alpha_{s}^{g h}(0) \simeq 4.74$ preferred). Recently, Huber has numerically obtained the decoupling solutions of the SDE for linear covariant gauges, varying its gauge fixing parameter between $0 \leq \xi \leq 0.2$ [313]. In each case, the couplings extracted from the ghost-gluon and 3-gluon vertices vanish as $Q^{2} \rightarrow 0$, albeit with a large gauge dependence in the intermediate $Q^{2}$ domain of a few $\mathrm{GeV}^{2}$.

\subsection{Lattice gauge theory}

Lattice calculations provide another important method for studying the behavior of the QCD coupling $\alpha_{s}$ in the IR regime. These studies connect with the SDE framework. Both approaches have greatly benefited from their complementarity. The basics of lattice QCD have been given in Section 3.9.4. Here, we will first describe the steps for typical lattice calculations of $\alpha_{s}\left(Q^{2}\right)$ in the IR. We will then discuss the results obtained by several groups which use the numerical lattice approach to compute the QCD coupling $\alpha_{s}$ in the nonpertubative infrared domain.

One method for obtaining $\alpha_{s}\left(Q^{2}\right)$ in the IR is to compute the static potential, as discussed in Sections 3.9.4 and 4.3. Another approach is to compute the coupling from vertices. In this case, just as for the SDE formalism, it amounts to determining correlation functions. Those were first calculated on the lattice by Mandula and Ogilvie [286]. In the following, we overview representative lattice methods to obtain $\alpha_{s}$ (valid in the IR as well as the UV domains). The examples are restricted to the pure gauge sector.

\section{An example of the lattice determination of $\alpha_{s}^{g h}$}

As discussed in Section 4.4, $\alpha_{s}\left(Q^{2}\right)$ can be defined from the ghost-gluon vertex, see Eq. (4.25). To compute the ghost and gluon propagator dressing functions, discretized ghost and gluon fields need to be defined. The discretized gluon field $\mathcal{A}_{\vec{\mu}}^{c}(x)$, where $c$ is the color index and $\vec{\mu}$ a link direction, may be defined as the difference between a link 
and its adjoint:

$$
\mathcal{A}_{\vec{\mu}}^{c}(x) \equiv \frac{1}{2 i}\left(U_{\vec{\mu}}(x)-U_{\vec{\mu}}^{\dagger}(x)\right) \underset{a \rightarrow 0}{\longrightarrow} 2 a \sqrt{\pi \alpha_{s}^{\text {bare }}} A_{\mu}^{c}(x)
$$

The continuum limit $a \rightarrow 0$ corresponds to the physical gluon field $A_{\mu}^{c}$. The discretization of space-time induces a finite difference $\mathcal{A}_{\vec{\mu}}^{c}(x)-2 a \sqrt{\pi \alpha_{s}^{b a r e}} A_{\mu}^{c}(x)$. This creates unphysical tadpole contributions proportional to $\alpha_{s}^{b a r e}$ or to $a^{3}$. Those can be eliminated by a redefinition of the link variable or of the action, see Refs. [106] or [230]. Other definitions of $\mathcal{A}_{\vec{\mu}}^{c}(x)$ have been used, and are equivalent -up to a multiplicative renormalization factor [314]. The gluon propagator from Eq. (3.53) is

$$
D_{\mu \nu}^{b c}(x-y)=\int D x A_{\mu}^{b}(x) A_{\nu}^{c}(y) e^{-i S} \equiv\left\langle A_{\mu}^{b}(x) A_{\nu}^{c}(y)\right\rangle
$$

It gives on the lattice

$$
\mathcal{D}_{\vec{\mu}}^{b c} \vec{\nu}(x-y)=\left\langle\mathcal{A}_{\vec{\mu}}^{b}(x) \mathcal{A}_{\vec{\nu}}^{c}(y)\right\rangle .
$$

Then, $\mathcal{D}_{\vec{\mu}}^{b c} \vec{\nu}(x-y)$ can be transformed to momentum space via a Fourier transform. This yields the gluon propagator dressing function $\mathcal{Z}\left(Q^{2}\right) \equiv \delta^{b c} \delta^{\mu \nu} \mathcal{D}_{\mu \nu}^{b c}\left(Q^{2}\right) Q^{2}$. The lattice ghost propagator is obtained, by definition, from the inverse of the Fadeev-Popov operator $(-\partial+A) A$; after discretization and evaluation on the lattice, it is Fouriertransformed to momentum space. The resulting ghost propagator dressing function is $\mathcal{G}=\delta^{b c} Q^{2} \mathcal{D}_{b c}^{G}\left(Q^{2}\right)$. The coupling $\alpha_{s}^{g h}\left(Q^{2}\right)$ is then extracted from Eq. (4.25) after applying the ghost and gluon propagator renormalization factors, $\widetilde{Z}_{3}$ and $Z_{3}$, respectively; see Section 3.5. The validity of the calculations can be verified by comparing the UV running of $\alpha_{s}^{g h}\left(Q^{2}\right)$ with the pQCD expectation.

The above procedure was applied by two different groups in [230] using different lattice simulations in the pure gauge sector. The results yield a $\mathrm{SU}(2)$ running coupling which matches the pQCD running at $Q^{2} \gtrsim 4 \mathrm{GeV}^{2}$ and freezes at $\alpha_{s}^{g h, S U(2)}(0)=5 \pm 1$. It is close to the $\mathrm{SDE} \mathrm{SU}(2)$ result found in [245]: $\alpha_{s}^{g h, S U(2)}(0)=5.24$. This result is extended to $\mathrm{SU}(3)$-color QCD by scaling it by $2 / 3$ : it becomes $\alpha_{s}^{g h}(0)=3.3 \pm 0.7$. Since $\alpha_{s}^{g h}\left(\mu^{2}\right)$ has been determined in [230] by matching the lattice result to the 2-loop pQCD expectation, the prediction is scheme-independent, but it is dependent on the order of truncation order of the perturbative $\beta$ series; it yields $\Lambda_{2-\text { loop }}=1.1 \mathrm{GeV}$ in the UV. 


\section{An example of the determination of $\alpha_{s}^{3 g}$}

Another approach, first proposed in Ref. [315], is to measure the coupling by calculating the 3- and 2-point gluon correlation functions,

$$
G_{3, \mu_{1} \mu_{2} \mu_{3}}\left(p_{1}, p_{2}, p_{3}\right) \equiv\left\langle A_{\mu_{1}}\left(p_{1}\right) A_{\mu_{2}}\left(p_{2}\right) A_{\mu_{3}}\left(p_{3}\right)\right\rangle
$$

and $D(p)=Z\left(p_{2}\right) / p_{2}$, respectively. The calculations are typically carried out in the Landau gauge and MOM RS. The 3-gluon vertex $G_{3}$ can be expressed in term of three gluon propagators $D$ and a 3 -point vertex function $\Gamma_{3}$ which is naturally identified with the coupling:

$$
G_{3, \mu_{1} \mu_{2} \mu_{3}}\left(p_{1}, p_{2}, p_{3}\right)=\Gamma_{3, \nu_{1} \nu_{2} \nu_{3}}\left(p_{1}, p_{2}, p_{3}\right) D_{\mu_{1} \nu_{1}}\left(p_{1}\right) D_{\mu_{2} \nu_{2}}\left(p_{2}\right) D_{\mu_{3} \nu_{3}}\left(p_{3}\right) .
$$

The vertex function is then extracted as:

$$
\Gamma_{3, \nu_{1} \nu_{2} \nu_{3}}\left(p_{1}, p_{2}, p_{3}\right)=G_{3, \mu_{1} \mu_{2} \mu_{3}}\left(p_{1}, p_{2}, p_{3}\right) \prod_{n=1}^{3} D_{\nu_{n}}^{-1 \mu_{n}}\left(p_{n}\right) .
$$

The coupling is normally defined at the symmetric Euclidean point, $\alpha_{s}\left(Q^{2}\right) \equiv \Gamma_{3}\left(p_{1}, p_{2}, p_{3}\right)$ for $p_{1}^{2}=p_{2}^{2}=p_{3}^{2}=Q^{2}$ and $p_{1}+p_{2}+p_{3}=0$. Such conditions correspond to the MOM scheme. In contrast, the minimal MOM scheme $\widetilde{\mathrm{MOM}}$ is preferred on the lattice, corresponding to the condition $p_{1}=p_{2}+p_{3}=0$.

\subsubsection{Lattice results for $\alpha_{s}$ in the IR}

Lattice calculations for $\alpha_{V}$ defined by the static Q- $\overline{\mathrm{Q}}$ potential leads to an IR-freezing coupling [206]. However, for $\alpha_{s}$ defined using Green's functions, both IR-vanishing and freezing solutions exist, as for the SDE calculations. Calculations by Bloch and collaborators [316], and by Furui and Nakajima [251] result in the scaling solution. On the other hand, a majority of calculations $[270,317,318,319,320]$, indicate that in the Landau gauge and MOM RS, $\alpha_{s}^{g h}$ vanishes when $Q^{2} \rightarrow 0$. Before concluding that these results correspond to the decoupling solution discussed in Section 4.4.3, one needs to explore more mundane possibilities. One is that $G_{3}$ has been obtained from Eq. (4.49) using the perturbative expression of the gluon propagator $D\left(Q^{2}\right)$, which diverges as $Q^{2} \rightarrow 0$. In this case, the IR-vanishing originates from an artificial divergence originating from $\mathrm{pQCD}$ which is irrelevant in the IR regime. Another possible reason is unphysical 
lattice finite-size effects [255]. Finally, the IR-behavior obviously depends on the choice of definition of the coupling, which can lead to diverging, freezing or vanishing $\alpha_{s}$. Nevertheless, it is now clear that the decoupling solution seen in the SDE framework also genuinely exists on the lattice [321], where it is dominant and where it was in fact first discovered [257].

Lattice data for the gluon and ghost propagators in $\mathrm{SU}(2)$ and $\mathrm{SU}(3)$ from Ref. [322] have been used by Aguilar, Binosi and Papavassiliou [323] to form $\alpha_{s}^{g h}$ from Eq. (4.25), and $\alpha_{g s e}$ using the pinch technique. As already discussed for other articles from these authors, a massive gluon formula is assumed for the gluon propagator at treelevel, $\Delta\left(Q^{2}\right) \propto 1 /\left[Q^{2}+m_{g}^{2}\left(Q^{2}\right)\right]$, rather than a massless propagator. This accounts for the freezing of their couplings in the IR rather than vanishing couplings. Assuming $m_{g}(0)=0.5 \mathrm{GeV}$, the Landau gauge coupling freezes at $\alpha_{s}^{g h}(0)=4.45$; for $m_{g}(0)=0.6$ $\mathrm{GeV}$, the freezing value is $\alpha_{s}^{g h}(0)=6.40$. The gauge-invariant coupling defined in the pinch technique freezes at $\alpha_{g s e}(0) \simeq 0.5$ for $m_{g}(0)=0.5 \mathrm{GeV}$ and $\alpha_{g s e}(0) \simeq 0.4$ for $m_{g}(0)=0.6 \mathrm{GeV}$.

Furui and Nakajima have used lattice gauge theory to calculate $\alpha_{s}^{g h}\left(Q^{2}\right)$ in the Landau gauge, $\widetilde{\mathrm{MOM}} \mathrm{RS}$ and the quenched approximation. Quarks are defined as domain wall fermions. The resulting coupling freezes at $\alpha_{s}^{g h}(0)=2.5 \pm 0.5$ [251]. In determining this value, the apparent IR-vanishing trend of $\alpha_{s}^{g h}$ was ignored since it was identified to be due to finite size effects [324].

Ayala and collaborators studied the influence of dynamical quarks on $\alpha_{s}^{\text {gh }}$ by performing quenched, and $n_{f}=2$ and $2+1+1$ unquenched lattice simulations [325]. These authors assume masses of order 20-50 MeV for the light quarks, $95 \mathrm{MeV}$ for the $s$ quark mass, and $1.51 \mathrm{GeV}$ for the $c$ quark mass. The Landau gauge and MOM RS are used. There is no direct influence of quarks on $\alpha_{s}^{g h}\left(Q^{2}\right)$ for a fixed dynamical gluon mass scale. However, as quarks change the value of $m_{g}$, they have an indirect influence. The authors obtain a freezing value of $\alpha_{s}^{g h}(0) \simeq 3.2 \pm 0.3$ for $m_{g}=0.5 \mathrm{GeV}$, and $\alpha_{s}^{g h}(0) \simeq 4.7 \pm 0.4$ for $m_{g}=0.6 \mathrm{GeV}$, both for $n_{f}=2+1+1$.

Recently Maas and collaborators have studied systematic lattice effects on the gluon and ghost propagators on $\alpha_{s}^{g h}\left(Q^{2}\right)$ in the Landau gauge [326]. The study was carried in 2, 3 and 4 dimensions and yields a solution for $\alpha_{s}^{g h}$ which vanishes in the IR.

The gluon and ghost propagators and other correlation functions have been evaluated on the lattice in many analyses, but without providing the IR-behavior of $\alpha_{s}$. An example of such results is shown in Fig. 4.5. In Ref. [327] the propagators have been obtained in the interpolating gauge in order to test the gauge dependence of the correlation 


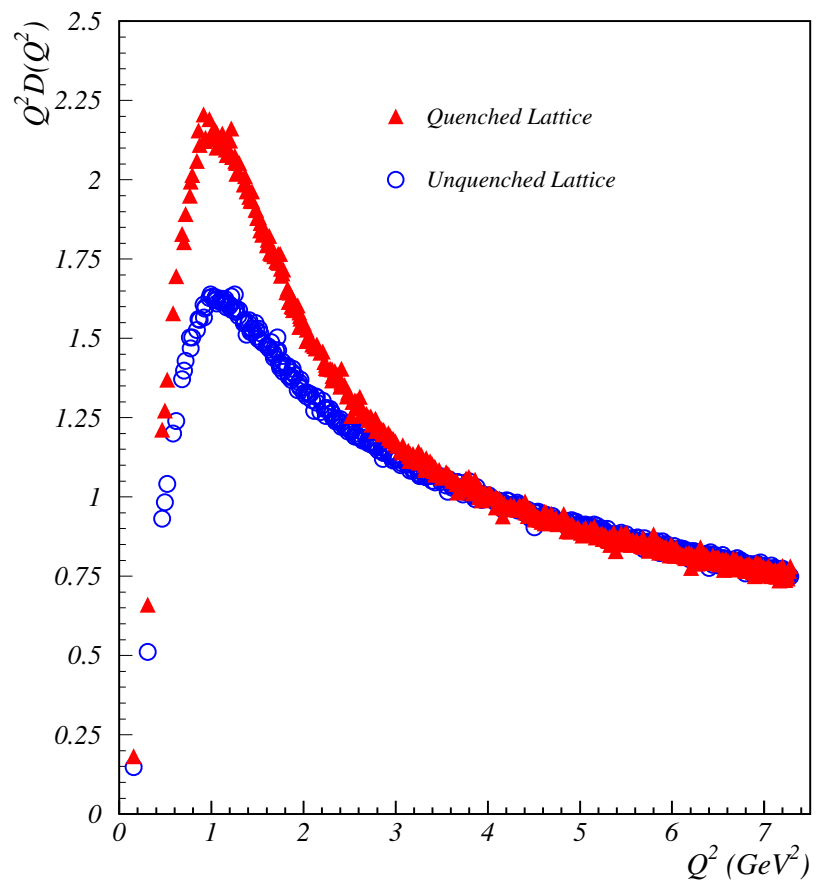

Figure 4.5: The gluon dressing function $Z\left(Q^{2}\right)=Q^{2} D\left(Q^{2}\right)$, from quenched and full lattice calculations from Bowman et al., Ref. [330].

functions. The authors obtained propagators compatible with the Kugo-Ojima and the Gribov-Zwanziger scenarios, regardless of the choice of gauge. In [328] it is shown that the gluon propagator in $3 \mathrm{D}$ and $4 \mathrm{D}$ is non-zero and finite in the IR, leading to an IRfinite coupling. The calculations are done in $\mathrm{SU}(2)$-color but the conclusion extends to $\mathrm{SU}(3)_{C}$. Indeed, a quenched calculation done in the Laplacian gauge for different numbers of colors $N_{C}(\mathrm{SU}(2), \mathrm{SU}(3)$ and $\mathrm{SU}(4))$ shows little dependence of the gluon propagator on $N_{C}$ [329]. This is compatible with the expected $1 / N_{C}$ dependence for the coupling because $\alpha_{p Q C D}$ factors the expressions of $\alpha_{s}$ defined from propagators (Eqs. (4.25), (4.41) and (4.42)), and that in the quenched approximation, $\alpha_{p Q C D} \propto 1 / \beta_{0} \propto N_{C}$.

Most of the propagator studies cited above are done in quenched QCD and Landau gauge. Calculations including dynamical quarks have also been been carried out, showing clear effects on the propagators from quark loops, but preserving the qualitative features obtained in quenched QCD [330]. Calculations in other gauges also exist and display similar features as the Landau gauge calculations, see e.g., Ref. [329] for calculations done in the Laplacian gauge. Discretization, finite lattice size and tadpole effects were studied in Ref. [331].

The coupling $\alpha_{s}\left(Q^{2}\right)$ can be also obtained from the static potential. In recent results 
by Horsley and collaborators [332], $\alpha_{V}\left(Q^{2}\right)$ and its $\beta$-function are computed on a lattice using numerical stochastic perturbation theory; see the reviews in Refs. [333, 334] and Section 4.8. The perturbative part of the static potential, $4 \alpha_{V} / 3 r$, is calculated from Wilson loops. In the quenched results, $\alpha_{V}\left(Q^{2}\right)$ diverges in the IR at $Q \simeq 0.7 \Lambda_{\overline{M S}}$. When quarks are included, $\alpha_{V}\left(Q^{2}\right)$ freezes around $Q \simeq 0.8 \Lambda_{\overline{M S}}$ with $\alpha_{V}(0) \simeq 0.52$. The calculations are performed for $\mathrm{SU}(3)_{C}$ and include massless $\mathrm{u}$ and d quarks.

Finally, another method is to use the Schrödinger functional; see Section 3.9.4. It leads to a coupling in good agreement with pQCD down to $Q^{2} \simeq 0.4 \mathrm{GeV}^{2}$, but it diverges in the IR as $e^{m / Q}[335]$.

\subsection{Functional renormalization group equations}

The functional renormalization group (FRG) framework is similar to the SDE approach. The central objects are Green's functions (although, only dressed ones, while SDE must include both bare and dressed functions) and the method provides an infinite system of "flow equations", or "exact renormalization group equations", which fully describe the underlying quantum field theory in continuous Euclidean space-time. Thus, the FRG equations and SDE must in principle share the same solutions. In practice, the different approximations needed to solve the FRG equations and the SDE can introduce differences. Those can be used to assess the uncertainties on the solutions. Thereby, just like SDE and Lattice benefited from their connection and complementarity, the FRG approach provides yet another complementary framework.

The starting point of the FRG method is to define an IR cut-off $k$ above which the theory is well understood and perturbatively calculable. Then the value of $k$ is lowered to access the nonperturbative domain. The cut-off takes the form of a regulator added to the classical action. In effect, it adds a large, momentum-dependent, mass to the field, suppressing the low modes of momenta below $k$ [250]. A flow equation for the Green's functions expresses how those depend on $k$ [336]. This flow equation is obtained by first partially deriving the generating functional of the quantum field theory which describes its dependence with respect to $k$. This yields the flow equation for the generating functional $\Gamma_{k}[\phi]$

$$
\frac{\partial}{\partial k} \Gamma_{k}[\phi]=\frac{1}{2} \operatorname{Tr} \frac{\frac{\partial}{\partial k} R_{k}}{\Gamma_{k}^{(2)}[\phi]+R_{k}},
$$

where $R_{k}$ is the regulator which decouples the infrared modes with momentum below 
the sliding scale $k$ and $\Gamma_{k}^{(2)}$, the second functional derivative, is the full inverse propagator modified by the presence of the regulator $R_{k}$. The trace stands for the sum over momenta, fields and internal indices. The Wetterich equation (4.50) is an exact flow equation for $\Gamma_{k}[\phi]$ which has a one-loop structure, in contrast with the multi-loop diagram structure characteristic of perturbation theory.

The system of flow equations for the Green's functions are obtained by successively deriving the flow equation of the generating functional with respect to the source or field terms in the action. Once the $k \rightarrow 0$ limit is reached, the cut-off disappears and all the slow modes of the quantum field theory are integrated out in the solution. One thus has an analytic, nonperturbative method for investigating strongly interacting quantum field theories. The FRG method is particularly relevant to the study of running couplings since they can be defined from the Green's functions as in other approaches.

The FRG equations thus provide the correct RG-scaling expected from the theory. As verification, the pure Yang-Mills expectation for the UV running of $\alpha_{s}\left(Q^{2}\right)$ has been recovered within the FRG framework [337]. In practice, the infinite system of equations must be truncated. As it is the case of the SDE formalism, the truncation introduces uncertainties which can be difficult to fully assess. Furthermore, multiple choices of regulator are possible, which introduces some arbitrariness. For a review of the FRG method and a discussion of its predictions for the running QCD coupling in the IR, see e.g., Ref. [338].

Initial FRG studies which were performed down to the scale $\sim \Lambda$ found an IR divergent solution of the gluon propagator, in agreement with the scenario of IR slavery; see Refs. [211, 339]. However, since the $k \rightarrow 0$ limit was not reached, the nonperturbative effects were not fully accounted for. More recent FRG investigations have now invalidated the IR slavery conclusion. The ghost and gluon propagators have been studied within the FRG formalism in the pure field sector, using Landau gauge and 4D Euclidean space [249, 250], assuming the scaling solution as observed in the lattice and SDE frameworks (Section 4.4.3). They yield a similar value of the $I R$ exponent: in [249], it is found that $\kappa^{\prime} \simeq 0.52$, which is compatible with the SDE value of 0.595 , once the dependence of $\kappa^{\prime}$ with the choice of cut-off implementation is accounted for. The range $0.539 \leq \kappa^{\prime} \leq 0.595$, with a strong preference for the maximum value $\kappa^{\prime}=0.595$ was obtained in Ref. [250]. The resulting freezing value of the ghost-gluon coupling is $\alpha_{s}^{g h}(0)=2.972$. All of these results are consistent with a freezing of the coupling due to IR ghost dominance and the Kugo-Ojima criterion. The ghost and gluon propagators and the coupling $\alpha_{s}^{g h}\left(Q^{2}\right)$ have also been studied using the FGR approach in [248]. These 
calculations have been also performed within the pure field sector using Coulomb gauge and a focus on the scaling solution. The resulting solution leads to a unique freezing coupling, although its freezing value is not specified. The solution yields $\kappa^{\prime}=0.35$, in agreement with Ref. [210] using a variational approach to the Yang-Mills Schrödinger equation, and with the first solution obtained using the SDE in [235].

The behavior of the running coupling is specifically addressed using FRG in Ref. [337] within the pure gauge field sector for both $\mathrm{SU}(2)_{C}$ and $\mathrm{SU}(3)_{C}$. The predicted coupling freezes in the IR at the value of $7.7 \pm 2$ for $\mathrm{SU}(3)_{C}$ and 11.3 for $\mathrm{SU}(2)_{C}$. The calculations were performed in the Landau gauge. The inclusion of quarks is expected to lower the freezing values [337], in agreement with the discussion in Section 4.4.7.

\subsection{The Gribov-Zwanziger approach}

Gribov was the first to point out the effects of gauge-fixing in a Yang-Mills theory [155]. Those are directly relevant to the coupling when it is defined using Green's functions. The coupling is invariant under the local gauge symmetry given by $\delta_{\omega} A_{\mu}^{a}=$ $-D_{\mu}^{a b} \omega^{b}$ (with $a, b$ color indices). However, the standard gauge-fixing relations, such as the Coulomb, Feynman or Landau gauges, are not enough to uniquely specify the gauge field. The vanishing eigenvalues of the Faddeev-Popov operator generate copies of the fields; i.e., physically different fields which are related by gauge transformations still exist, even after fixing the gauge. To avoid these copies in the path-integral formalism, Gribov proposed to restrict the Faddeev-Popov operator to an integration region where the operator is strictly positive (the so-called "first Gribov" region). To implement such a restriction, Gribov introduced a non-local operator. Even with this prescription, copies still exist within the first Gribov region [340]. This has prompted poposals to restrict the integration to an even smaller domain, the "fundamental modular region", which is truly free of Gribov copies. It was eventually found however, that the remaining copies appear to have no influence on the solutions of the theory [237, 285, 341, 342], which is consistent with the finding from lattice gauge theory, namely that the effect of Gribov copies ("Gribov noise") is negligible [314, 343].

The non-locality of Gribov's operator limits the formalism to semi-classical calculations. A local renormalizable Lagrangian restricting the integration region to the first Gribov region was later proposed by Zwanziger [207], enabling loop calculations. For a review of the Gribov-Zwanziger approach, see Ref. [333].

Since the ghost propagator is the inverse of the Faddeev-Popov operator, the ghost 
propagator has no pole in the Gribov approach, except on the horizon of the Gribov region, which corresponds to the $Q^{2} \rightarrow 0$ limit. In this case, the ghost propagator diverges, following a $1 / Q^{4}$ dipole form. Gribov found that when the integration of the generating functional, or any correlation function, is restricted to the first Gribov region, the Gribov mass generated by the copies suppresses the tree-level gluon propagator in the Landau gauge, which should then take the IR form:

$$
D_{\mu \nu}^{b c}(q)=\delta^{b c}\left(\eta_{\mu \nu}-\frac{q_{\mu} q_{\nu}}{q^{2}}\right) \frac{q^{2}}{q^{4}+m_{g r}^{4}}
$$

where $m_{g r}$ is the Gribov mass. With this form, the gluon propagator vanishes in the IR, wheras as noted above, the ghost propagator diverges as $1 / Q^{4}$. This behavior is distinct from its $1 / Q^{2} \mathrm{UV}$-behavior. This scenario, the Gribov-Zwanziger approach, results in an IR freezing of $\alpha_{s}$ analogous to the scaling solution seen in the SDE and lattice studies; see Section 4.4.3 and Ref. [344]. However, the predicted $I R$ exponent is $\kappa^{\prime}=1$ in this approach, differing from that of SDE $\left(\kappa^{\prime} \simeq 0.595\right)$. The IR-freezing value in this scenario was calculated for the ghost-gluon coupling by Gracey to be $\alpha_{s}^{g h}(0)=16 / 3 \pi \simeq 1.70$ (Landau gauge, $\overline{M S}$, one loop calculation) [345].

The analytical properties of the calculations using the Gribov-Zwanziger approach allows one to investigate the underlying reason for the freezing of the QCD coupling, at least at one loop: As one expects on general dimensional considerations, the freezing occurs because all dimensionful quantities cancel each other in the IR, so the coupling becomes scale invariant. The mechanism for such a cancellation has its origin in the interplay of the anti-commutating Faddeev-Popov ghosts and the commutating "Zwanziger ghosts". Those arise from enforcing the Gribov region with the local Zwanziger Lagrangian. More recent 2-loop calculations of the gluon and ghost propagators are available $[208,346]$. Although the original Gribov-Zwanziger approach leads to the scaling solution, it should be noted that the decoupling scenario also appears to be possible in an extended Gribov-Zwanziger approach, see Refs. [263, 264].

The Gribov-Zwanziger scenario may appear gauge-dependent since gauge-fixing procedures without Gribov copies exist, such as the axial or Laplacian gauges; see the related discussion in Ref. [347]. The light-cone gauge $A^{+}=0$ is also free of ghosts. In addition, there are no ghosts using the stochastic gauge-fixing quantization method (see below) which, in fact, was introduced in the QCD propagators/coupling context to circumvent the Gribov copy problem. However, in spite of the apparent gauge dependence of the Gribov-Zwanziger scenario, equivalent mechanisms are found in the ghost-less 
frameworks, as we will see in the next section.

\subsection{Stochastic quantization}

Stochastic quantization provides a method to obtain the Green's functions of an Euclidean field theory [348]. It was first used by Zwanziger [237, 349] as a mean to obtain and solve the SDE without the Faddeev-Popov procedure, thus circumventing the Gribov copy problem just discussed.

The formalism of stochastic quantization is based on a diffusion equation analogous to the Fokker-Planck equation of statistical mechanics. The diffusion equation acts within the space of gauge field configurations; it controls the probability distribution $P\left(A_{\mu}\right)$ of the gluon field $A_{\mu}$, just as the Fokker-Plank equation controls the spatial distribution of diffusing bodies. The distribution $P\left(A_{\mu}\right)$ is the weight of the QCD's Euclidean generating functional, $P\left(A_{\mu}\right)=e^{-S}$; and the nomenclature "stochastic" comes from the weighted random walk of $P\left(A_{\mu}\right)$ characterized by the diffusion equation. The drift force term in the diffusion equation is given by the functional derivative of the YangMills action, $K_{Y M \mu}^{a}=-\delta S_{Y M} / \delta A_{\mu}^{a}$. The field solution of the equation is also the solution of the Yang-Mills theory. There is no specific choice of gauge in this approach. Instead of a single gauge-fixing which introduces the Gribov copies, a supplemental drift force term in the form of infinitesimal gauge transformation, $K_{g t} \underset{\mu}{a}=a^{-1}\left(\partial_{\mu} \delta^{a c}+f^{a b c} A_{\mu}^{b}\right) \partial \cdot A^{c}$, is added to the diffusion equation. The coefficient $a$ is a free-parameter akin to a gaugefixing term which controls the balance between the Yang-Mills drift force $K_{Y M}$ and a gauge restoring force $K_{g t}$. This approach reproduces perturbative QCD results in the UV [350]. Furthermore, in the case of Landau gauge $(a \rightarrow 0)$, stochastic quantization yields the same scaling solution in the IR as the SDE using the Faddeev-Popov method (Section 4.4), lattice (Section 4.5) and FRG frameworks (Section 4.6), with a compatible IR exponent $\kappa^{\prime}$ for the gluon propagator [266, 349].

It has been found recently that the decoupling scenario is also a solution of the stochastic quantization equations in the Landau gauge [266]. Zwanziger has shown that the stochastic quantization formalism, constrained to the Landau gauge limit, generates the Fadeev-Popov theory together with the Gribov gauge-fixing prescription [237]. Furthermore, the diffusion equation, which can be solved exactly in the Landau gauge limit, has the same solution as the Fadeev-Popov theory restricted to the first Gribov region [342]. Thus, $K_{g t}$ provides an additional term which removes the Gribov copy problem $[237,333]$. 
Zwanziger has also derived the stochastic quantization SDE using the diffusion equation. Using truncation, the diffusion equation was solved approximately, but nonperturbatively, by assuming that the gluon propagator follows a power law in the IR. It was found by Zwanziger [349], and confirmed by Llanes-Estrada and Williams [266], that the gluon propagator contains a longitudinal component which becomes large and dominant

in the IR. The transverse gluon propagator is IR-suppressed as $\left(Q^{2}\right)^{-1+2 k^{\prime}} \simeq\left(Q^{2}\right)^{0.04}$ due to longitudinal gluon loops, whereas the longitudinal gluon propagator is IR-enhanced as $\left(Q^{2}\right)^{-1-k^{\prime}} \simeq\left(Q^{2}\right)^{-1.52}$. This shows that in the stochastic quantization approach, longitudinal gluons take the place of ghosts. Although the longitudinal gluon propagator vanishes as $a$ in the Landau gauge limit, it still acts as the equivalent of a ghost since vertices behave as $1 / a$, yielding an overall finite result. This leads to the same picture of confinement as already discussed: confinement would result from the enhanced long-distance propagation of longitudinal gluons ( $i$. e., ghosts in the other frameworks), while the transverse gluons (i.e., the gluons in the other frameworks) decouple at long distances because of longitudinal gluons (ghosts) loops, and become irrelevant. This implies an IR-freezing behavior for $\alpha_{s}$. The inclusion of quark loops is expected to preserve this feature and to play no role in the IR as long as they are not massless [342].

Pawlowski and collaborators [351] have studied the ghost and gluon propagators in $\mathrm{SU}(2)_{C}$, in 2, 3 and 4 dimensions and in the Landau gauge using the stochastic quantization formalism on the lattice, and, as for most of the other lattice results, have obtained the decoupling solution with an IR-vanishing coupling [351].

\subsection{Analytic and dispersive approaches}

We have discussed gauge-dependent and RS-dependent couplings, starting with the SDE approach. As already noted, these arbitrary dependences are not a major obstacle, since in general $\alpha_{s}$ is not an observable. Nevertheless, one can try to restore the definition of $\alpha_{s}$ to a status close to an observable, as in the case of effective charges such as $\alpha_{V}$ or $\alpha_{g 1}$, by demanding that it satisfies causality, i.e., that it is analytic in the complex $Q^{2}$-plane, except for branch points or cuts on the real time-like axis. Since the Landau pole on the space-like-axis violates causality, demanding analyticity and the causality of $\alpha_{s}$ regularizes the Landau singularity. Such "analytic" and "dispersive" approaches were first proposed in the context of QED by Redmond [352] and then applied to QCD [160, 353, 354]. Early work by Sanda [160], in which an analytic behavior is forced on $\beta\left(\alpha_{s}\right)$ using a Borel summation technique, yielded a coupling freezing at $\alpha_{s}(0) \simeq 4$. For 
reviews of the analytic and dispersive approaches, see [2, 144, 355]. These approaches are also discussed in the context of the SDE in [228].

\subsubsection{Analytic approach}

The Shirkov et al. "analytic" approach results in the folding of QCD nonperturbative effects into the coupling $[353,354,356]$. The starting point is to define the analytic coupling $\alpha_{a n}\left(Q^{2}\right)$ from a "spectral density" $\rho(\nu)$, following the Källén-Lehman relation:

$$
\alpha_{a n}\left(Q^{2}\right)=\frac{1}{\pi} \int_{0}^{\infty} d \nu \frac{\rho(\nu)}{\nu+Q^{2}}
$$

where the expression of $\rho(\nu)$,

$$
\rho(\nu)=\Im m\left(\alpha_{p Q C D}^{(l)}(-\nu-i \varepsilon)\right) \alpha_{p Q C D}^{(l)}(-\nu-i \varepsilon)
$$

depends on $\alpha_{p Q C D}^{(l)}$ at loop order $\beta_{l}$. The incorporation of the spectral function of Eq. (4.53) into Eq. (4.52) leads to a Kramer-Krönig type of relation for the coupling, which demonstrates its analyticity/causality property. The expression of $\alpha_{a n}$ at order $\beta_{0}$ is:

$$
\alpha_{a n}^{(0)}\left(Q^{2}\right)=\frac{4 \pi}{\beta_{0}}\left(\frac{1}{\ln \left(Q^{2} / \Lambda^{2}\right)}+\frac{\Lambda^{2}}{\Lambda^{2}-Q^{2}}\right) .
$$

The result of regulating the Landau pole in the IR domain leads to an effective $\alpha_{a n}\left(M_{Z}^{2}\right)$ compatible with the world data for $\alpha_{s}\left(M_{Z}^{2}\right)$ [357]. The dependence on $\Lambda$ cancels at $Q^{2}=0$ and we have $\alpha_{a n}^{(0)}(0)=4 \pi / \beta_{0}$. This value holds at all orders [354, 358, 359]. Near the Landau pole, $\alpha_{a n}^{(0)}\left(\Lambda^{2}(1+\varepsilon)\right)=2 \pi / \beta_{0}+\mathcal{O}\left(\epsilon^{2}\right)$. As can be seen in Fig. 4.6, $\alpha_{a n}\left(Q^{2}\right)$ does not freeze in the IR but does stay finite. The term $\Lambda^{2} /\left(\Lambda^{2}-Q^{2}\right)$ in Eq. (4.54) is a nonperturbative power law contribution since it is independent of higher order perturbative terms in $\beta_{l}$.

The procedure described above is extendable to higher orders, [2, 358], with the same caveat as for the perturbative coupling: the solutions for $\beta_{2}$ and higher orders are either numerical or approximately analytic solutions. The solution at order $\beta_{1}$ involves the Lambert function; see Section 3.2. The resulting higher-order solutions $\alpha_{a n}^{(l)}\left(Q^{2}\right)$ are numerically close to $\alpha_{a n}^{(0)}\left(Q^{2}\right)$. This is not surprising since all $\alpha_{a n}^{(l)}\left(Q^{2}\right)$ have the same limit at $Q^{2}=0$; in addition, they have a similar structure at $Q^{2} \gg \Lambda^{2}$ in the sense that they differ only via perturbative terms $\beta_{l}$. If nevertheless, one needs a coupling beyond 


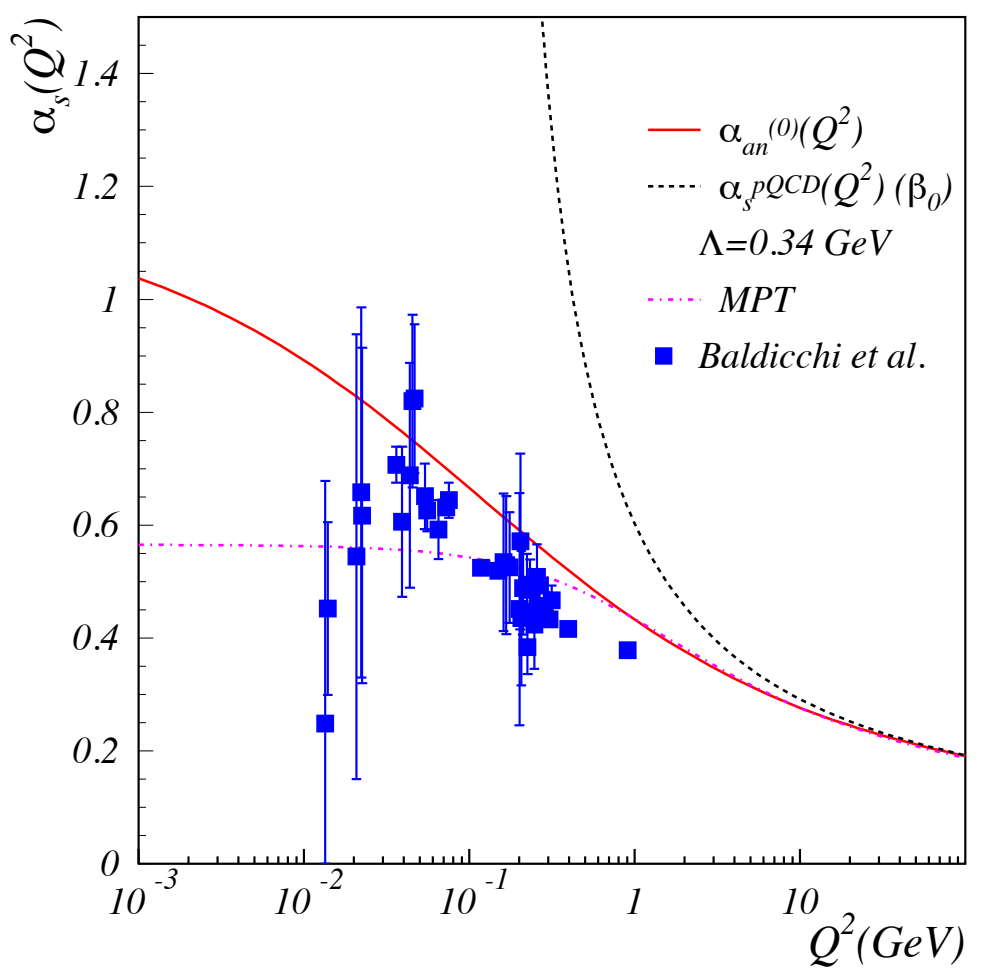

Figure 4.6: The strong coupling $\alpha_{a n}^{(0)}\left(Q^{2}\right)$ in the analytic approach (continuous line), Eq. (4.54). It can be compared to the pQCD expectation at leading order (dashed line) and to the experimental extraction using the analytic definition of the strong coupling [166]. The value of $\Lambda$ was taken in the $\overline{M S}$ scheme. The dotted line is the massive analytic perturbation theory prediction for $\Lambda=0.315 \mathrm{GeV}$ and a glueball mass parameter $m_{g l}=0.995 \mathrm{GeV}$.

order $\beta_{0}$, convenient approximations are given in Refs. [360, 361].

The analytic approach of Shirkov yields good pQCD results for large $Q^{2}$ [358] and the additional $\Lambda^{2} /\left(\Lambda^{2}-Q^{2}\right)$ term generates, for large $Q^{2}$, a series $(\Lambda / Q)^{2 n}$ as expected from the OPE [355, 362, 363, 364]; However, the results do not encompass all nonperturbative effects. For example, a meaningful comparison with the strong coupling $\alpha_{g 1}\left(Q^{2}\right)$, obtained from the Bjorken sum, is not possible, see Section 5.3. In addition, the decay branching ratio of the $\tau$ lepton into non-strange hadrons evaluated with $\alpha_{a n}$ is significantly underestimated [359, 365, 366].

The analytic approach was used by Baldicchi et al. to experimentally extract $\alpha_{s}\left(Q^{2}\right)$ for $0.013<Q^{2}<0.904 \mathrm{GeV}^{2}$ from quarkonium spectroscopy [166], see Fig. 4.6. The result is obtained in a model-dependent way of using the Bethe-Salpeter formalism. The ansatz based on the Wilson loop correlator used is reminiscent of the potential approach; see Section 4.3. It incorporates a Coulomb-like one-gluon exchange term proportional 
to $\alpha_{s}\left(Q^{2}\right)$ and a confining term proportional to $\sigma$, the string tension. The meson mass spectrum is calculated with $\alpha_{s}\left(Q^{2}\right)$ using parameters such that experimental mass values are reproduced. The authors concluded that the meson spectrum is better reproduced with an analytic-type coupling without freezing rather than with a freezing coupling constant [367]. This conclusion is model-dependent since it depends on the methodology chosen to compute the meson spectrum. Indeed, other approaches in which $\alpha_{s}$ freezes in the IR reproduce the meson spectrum as well, as discussed in Sections 3.9.6 and 4.3.2. The low- $Q^{2}$ results of Baldicchi et al. are compatible with a finite $\alpha_{s}(0)$ value lower than expected from $\alpha_{a n}$ (Eq. 4.54), or even a vanishing $\alpha_{s}(0)$. The large experimental and theoretical uncertainties prevent a firm conclusion. The coupling is normalized in the UV so that the 3-loop value $\alpha_{a n}^{(2)}\left(M_{Z}^{2}\right)$ corresponds to the average world data for $\alpha_{\overline{M S}}$. We also note that the experimentally extracted $\alpha_{s}$ should match the $V$-scheme, so the choice to match to $\alpha_{\overline{M S}}$ may not be optimal. G. Ganbold reproduced the experimental coupling $\alpha_{s}\left(Q^{2}\right)$ of Baldicchi et al., and the meson mass spectrum with its Regge behavior, starting from IR-finite quark and gluon propagators within a Bethe-Salpeter framework [368].

One can also improve the pQCD series by suppressing the Landau pole and using the analytic approach. This method is known as analytical perturbation theory (APT). For example, $\alpha_{a n}$ has been applied to the Bjorken sum rule perturbative expression, Eq. (3.40), allowing one to push down the pQCD limit close to the domain of validity of chiral perturbation theory [369].

Efforts have also been made to extend the validity of chiral perturbation theory predictions, see e.g., [179], and to systematically improve the semi-classical approximation provided by light-front holographic QCD [124]. Bridging the high and low $Q^{2}$ domains could provide a convenient analytical description of QCD using hadronic degrees of freedom at low $Q^{2}$ and partonic ones at large $Q^{2}$. Progress towards such bridging has been achieved recently [125].

Improved perturbation theory would also allow for more reliable extractions of higher-twist terms; However, their meaning needs to be reinterpreted since some of their effects are folded in $\alpha_{a n}$. The APT formalism has been extended by Bakulev and collaborators in [370].

In addition to the approach just described, analytic forms for $\alpha_{s}$ can be obtained using effective charges and other methods. For example, Nesterenko et al. constrained the full $\beta$-function to be analytical $[362,371]$, yielding an "analytic invariant charge" 
similar to $\alpha_{a n}\left(Q^{2}\right)$, Eq. (4.54). At $\beta_{0}$ it reads:

$$
\alpha_{a i c}\left(Q^{2}\right)=\frac{4 \pi}{\beta_{0}}\left(\frac{1}{\ln \left(Q^{2} / \Lambda^{2}\right)}-\frac{\Lambda^{2}}{Q^{2} \ln \left(Q^{2} / \Lambda^{2}\right)}\right)
$$

In contrast to $\alpha_{a n}\left(Q^{2}\right), \alpha_{\text {aic }}\left(Q^{2}\right)$ diverges as $Q^{2} \rightarrow 0$. This small- $Q^{2}$ behavior could accounts for the linear confinement term of the static potential; see the discussion in Section 4.3. Alternatively, the divergent behavior can be suppressed by including effects due to the pion mass [372], yielding a value of $\alpha_{\text {aic }}(0)=0.47$ for $\Lambda=0.623 \mathrm{GeV}$ and $n_{f}=2$. This approach has been linked to the phenomenology of instantons [373]. The compatibility of Nesterenko's coupling to the Schwinger-Dyson formalism and its consequences for chiral symmetry breaking are discussed in Ref. [374], where IR divergent constituent quark masses are taken as a manifestation of quark confinement, and the value of $\Lambda=0.880 \mathrm{GeV}$ is constrained in order to recover the pion decay constant.

Alekseev et al. has introduced a "synthetic coupling" [375] which builds on the concept of $\alpha_{a n}$, Eq. (4.54), by adding nonperturbative pole terms to the coupling, or equivalently $\delta$-functions in the spectral density $\rho(\nu)$. These terms simulate the static linear confinement potential and the dynamically generated gluon effective mass discussed in Sections 4.3 and 4.4.4. The extended definition at order $\beta_{0}$ is:

$$
\alpha_{\text {syn }}\left(Q^{2}\right)=\frac{4 \pi}{\beta_{0}}\left(\frac{1}{\ln \left(Q^{2} / \Lambda^{2}\right)}+\frac{\Lambda^{2}}{\Lambda^{2}-Q^{2}}+\frac{c \Lambda^{2}}{Q^{2}}+\frac{(1-c) \Lambda^{2}}{Q^{2}+m_{g}^{2}}\right),
$$

where $c>1$ is a dimensionless parameter and the gluon effective mass is $m_{g}=\Lambda / \sqrt{c-1}$. The term $c \Lambda^{2} / Q^{2}$ provides the linear binding term in the static potential with string tension $\sigma=8 \pi c \Lambda^{2} / 3 / \beta_{0}$. Expressions exist up to order $\beta_{3}$.

The massive analytic perturbation theory (MPT) is another analytic approach by Shirkov [376] in which the suppression of the Landau pole is achieved by the introduction of a glueball mass $m_{g l} \simeq 1 \mathrm{GeV}$, rather than by the $\Lambda^{2} /\left(\Lambda^{2}-Q^{2}\right)$ term in Eq. (4.54). The nonperturbative coupling is essentially obtained from the pQCD expression of $\alpha_{s}\left(Q^{2}\right)$ by substituting $Q^{2}$ by $Q^{2}+m_{g l}^{2}$. It results in an IR-freezing behavior; see Fig. 4.6. MPT was in particular used to improve the APT description of the IR experimental data for the Bjorken sum, Eq. (3.40), obtained at Jefferson Lab [70, 177]. The elimination of the Landau pole can also be linked to a glueball mass in background perturbation theory; see Section 4.10.

All of these related approaches are physically-motivated to suppress the Landau pole which is the artifact of the standard perturbative approach. Several versions of analytic 
couplings have been coded in Fortran by Ayala and Cvetic [377].

\subsubsection{Dispersive approach}

The "dispersive" approach was proposed by Y. L. Dokshitzer and collaborators [168, 169] to manage the presence of nonperturbative $\mu_{n}\left(Q^{2}\right) / Q^{n}$ power corrections expected from the OPE. The parameter $\mu_{n}\left(Q^{2}\right)$ depends logarithmically on $Q^{2}$; see e.g., Eq. (3.25). The goal of the resulting coupling $\alpha_{d m w}\left(Q^{2}\right)$ is to represent the strength of the strong interaction at large distances; it is thus parameterized by a set of phenomenological parameters which can be extracted from inclusive observables. Hence, the resulting coupling incorporates power corrections, as is the case for the effective charges discussed in Section 4.1, but it is designed to provide a universal coupling, without specific process dependence. The coupling $\alpha_{d m w}\left(Q^{2}\right)$ can therefore be related to $\alpha_{V}$ and to $\alpha_{g s e}$ defined from the gluon self-energy [226], see also Ref. [227]. As it is the case for $\alpha_{g s e}$, the coupling $\alpha_{d m w}(0)$ has an IR finite value of about 0.6 [168, 281]. Similar approaches are described in the references given in [61] (Sections 3.8 and 4.11) and [183] (Section 4.1.3). It is also closely related to Grunberg's concept of an effective charge (Section 4.1).

Dokshitzer, Marchesini and Webber have proposed an effective coupling which satisfies a dispersion relation:

$$
\rho\left(\nu^{2}\right)=\frac{d \alpha_{d m w}\left(\nu^{2}\right)}{d \ln \left(\nu^{2}\right)},
$$

where $\nu$ is a dispersive variable. Although the formalism uses standard massless gluon fields, $\nu$ plays a role equivalent to a small gluon mass ("dispersive mass") in the calculations. The Dokshitzer-Marchesini-Webber coupling is related to a perturbative coupling, with a definition close to the $V$-scheme and, like the effective charges of Sections 3.7.1 and 4.1 or $\alpha_{g s e}$ of Section 4.4.4, it can be viewed as a generalization of the Gell-MannLow QED coupling, since it is the coupling stemming from the propagator of a dressed gluon $[168,2]$. The coupling $\alpha_{d m w}\left(\nu^{2}\right)$ is also related to the analytic coupling $\alpha_{a n}$ which obeys the Källén-Lehman dispersion relation; see Eq. (4.52):

$$
\alpha_{a n}\left(Q^{2}\right)=Q^{2} \int_{0}^{\infty} \alpha_{d m w}\left(\nu^{2}\right) \frac{d \nu^{2}}{\left(\nu^{2}+Q^{2}\right)^{2}},
$$

or

$$
\alpha_{d m w}\left(\nu^{2}\right)=\operatorname{sinc}\left(\pi \frac{d}{d \ln \left(\nu^{2}\right)}\right) \alpha_{a n}\left(\nu^{2}\right) .
$$

Since $\alpha_{a n}$ is obtained from a dispersion relation, it has no Landau pole. Expanding the 
cardinal sine to first order yields $\alpha_{d m w}$ for small $\alpha_{a n}\left(Q^{2}\right)$ regime, i.e. in the UV:

$$
\alpha_{d m w}\left(\nu^{2}\right) \simeq \alpha_{a n}\left(\nu^{2}\right)-\frac{\pi^{2}}{6} \frac{d^{2} \alpha_{a n}\left(\nu^{2}\right)}{d \ln ^{2}\left(\nu^{2}\right)}+\ldots,
$$

This effective coupling is designed to provide the power corrections for inclusive observables as a function of a finite set of parameters to be determined phenomenologically. For example, the DIS structure function is obtained by calculating the relevant Feynman diagram amplitudes with gluons of effective mass $\nu$. At first order:

$$
F\left(Q^{2}, x_{f}\right)=\int_{0}^{\infty} \frac{d \nu^{2}}{\nu^{2}} \alpha_{d m w}\left(\nu^{2}\right) \dot{\mathcal{F}}\left(Q^{2}, x_{f}\right)
$$

with $\dot{\mathcal{F}} \equiv \partial \mathcal{F}\left(Q^{2}, x_{f}, \nu^{2}\right) / \partial \ln \left(\nu^{2}\right)$, the "characteristic function" of $F\left(Q^{2}, x_{f}\right)$. $\dot{\mathcal{F}}$ can be calculated perturbatively; it depends only on $x_{f}$ at first order, together with the power corrections $\mu^{2}\left(Q^{2}\right) / Q^{2}$. As suggested by the OPE, $F$ can also be written as:

$$
F\left(Q^{2}, x_{f}\right)=F^{P T}\left(Q^{2}, x_{f}\right)+\sum \frac{\mu_{2 p}\left(Q^{2}, x_{f}\right)}{Q^{2 p}}
$$

where $F^{P T}$ is the perturbative expression of $F$. The $O P E$ imposes the condition that $p$ is an integer, whereas the present approach allows it to also be a half-integer. The possible values of $p$ are predicted by the formalism. The power correction coefficients take the form:

$$
\mu_{2 p}=C_{1} A_{2 p}+C_{2} A_{2 p}^{\prime}+C_{3} A^{\prime \prime}{ }_{2 p}
$$

The coefficients $C_{i}$ are obtained for a specific process by calculating $\mathcal{F}$ in the pQCD $\mu^{2} / Q^{2} \rightarrow 0$ limit. The $Q^{2}$-dependent functions $A_{2 p}, A_{2 p}^{\prime}$ and $A^{\prime \prime}{ }_{2 p}$ must be obtained from measurements; however, they are universal. The pinch technique allows to form a gauge-independent formulation of $\alpha_{d m w}$ and to relate it to $\alpha_{g s e}$. This program has been applied to a large number of observables in addition to the applications provided in [168].

\subsection{Background perturbation theory}

Given the importance of nonperturbative effects at low- $Q^{2}$ and the successes of the static potential approach of Eq. (4.15), in which short- and long-distance forces are conveniently separated, several authors have proposed to formulate QCD with the gluon 
field separated as a perturbative part and an effective nonperturbative part [378]. Within this framework, namely background perturbation theory (BPT), Simonov computed the 1-loop coupling [212] in the pure field case. Three possible definitions of $\alpha_{s}$ have been considered, including one based on the static potential [212].

The gluon propagator in given by its perturbative Green's function in a nonperturbative background characterized by the QCD string tension $\sigma$. The nonperturbative background strongly influence the long-distance behavior of $\alpha_{s}$; it becomes finite and freezes at small $Q^{2}$-values. In effect, the background field introduces a mass term such that the argument of $\alpha_{s}\left(Q^{2}\right)$ becomes $Q^{2}+m_{2 g}^{2}$ in the IR; wheras the pQCD form is retained in the UV where the influence of the nonperturbative background field is negligible. This replacement is valid at all loop orders. For example, the 1-loop coupling can be cast into the same form as first put forth by Cornwall, Eq. (4.28):

$$
\alpha_{B P T}\left(Q^{2}\right)=\frac{4 \pi}{\beta_{0} \ln \left(\frac{m_{2 g}^{2}+Q^{2}}{\Lambda^{2}}\right)} .
$$

Here, rather than being a $Q^{2}$-dependent effective gluon mass, $m_{2 g}$ is a constant. However, it depends on the process considered $[379,380]$ : It can be related to the tension of the fundamental QCD string $\sigma_{f}: \sqrt{2 \pi \sigma_{f}} \sim 1 \mathrm{GeV}$ [212], or to the mass of a two-gluon bound-state glueball $M_{2 g}\left(0^{++}\right)$: the tension of the adjoint string connecting the two gluons, $m_{2 g} \simeq \sqrt{2 \pi \sigma_{a}} \simeq 2 \mathrm{GeV}$ [380].

In this formalism the power corrections (IR renormalon) are again folded into the coupling. An interesting insight is that the freezing value (or equivalently the value of $m_{2 g}$ ) is not universal, but depends on the embedding process, as is it is the case for the effective charges; see Sections 3.7 .1 and 4.1 . The regulator mass, $m_{2 g} \simeq 1-2$ $\mathrm{GeV}$-depending on the process considered-yields a range of freezing values between 0.37 and 0.60 for $\Lambda_{\overline{M S}}=0.34 \mathrm{GeV}$. The freezing of $\alpha_{B P T}$ is interpreted as due to the paramagnetic interaction of the gluon spin with the nonperturbative background field [380].

Badalian and collaborators [44] have also used the BPT to compute $\alpha_{B P T}$ in coordinate space. Given this coupling, the perturbative $1 / r$ static $Q-\bar{Q}$ potential can be produced $[44,381]$. The calculations are done up to 3 loops, where the values of $\Lambda_{B P T}=0.385 \mathrm{GeV}$ and $m_{2 g}=1.06 \mathrm{GeV}$ are obtained from the analysis of the bottomium fine structure. The corresponding freezing values, given in Table 4.1, depend on the loop order and the number of quark flavors. 


\begin{tabular}{|c|c|c|c|}
\hline loop & 1 & 2 & 3 \\
\hline \hline$n_{f}=1$ & 0.598 & 0.428 & 0.805 \\
\hline$n_{f}=3$ & 0.731 & 0.536 & 0.972 \\
\hline
\end{tabular}

Table 4.1: Values for $\alpha_{B P T}(0)$ in background perturbation theory for $\Lambda_{B P T}=0.385 \mathrm{GeV}$ (taken independently of $n_{f}$ ), $m_{2 g} \simeq 1 \mathrm{GeV}$, and various loop orders and $n_{f}$ values.

\subsection{Optimized perturbation theory}

Mattingly and Stevenson have applied OPT (see Section 3.8) at $3^{\text {rd }}$ order to the $e^{+} e^{-}$total cross-section $R_{e^{+} e^{-}}$[382]. This produces an IR-finite optimized coupling $\alpha_{O P T}$. Solving the OPT equations and demanding RS-independence yield a coupling which freezes below $Q^{2} \simeq 0.1 \mathrm{GeV}^{2}$ at the value:

$$
\alpha_{O P T}=\pi \frac{-\beta_{1}+\sqrt{\beta_{1}^{2}-336 \beta_{0}^{2} c}}{24 \beta_{0} c}=0.826
$$

with $c=10.911$. This result is obtained at $3^{r d}$ order in the OPT and for $n_{f}=2$. Comparison between the results at $2^{\text {nd }}$ and $3^{\text {rd }}$ orders suggests that the convergence of $\alpha_{O P T}$ is good, with $\alpha_{O P T}^{(3)}\left(Q^{2}\right)<\alpha_{O P T}^{(2)}\left(Q^{2}\right)$.

\subsection{Quark-hadron duality}

This approach by Courtoy and Liuti [383], is based on global quark-hadron duality, a phenomenon discovered in 1970 by Bloom and Gilman [126, 127]. Bloom and Gilman observed a remarkable similarity between the unpolarized proton structure function $F_{2}^{p}$ when it is measured in DIS, and its average when it is measured in the resonance region: in effect, the DIS measurement appears as the average of the resonance contributions. This matching is interpreted as limiting the size of the higher-twist contributions to $F_{2}$. Courtoy and Liuti have noted that the large- $x_{f}$ resummation contribution to $F_{2}^{p}$, corrected for nonperturbative effects, requires the knowledge of $\alpha_{s}$ in the IR [383]. Thus the evolution of $\alpha_{p Q C D}\left(Q^{2}\right)$ near $Q^{2} \simeq 1 \mathrm{GeV}^{2}$ must be regulated in order to explain parton-hadron duality. The freezing behavior of the coupling is assumed [383] and its freezing value is determined to be $\alpha_{s}(Q \lesssim 1 \mathrm{GeV})=0.50 \pm 0.08$ ( $\left.\overline{\mathrm{MS}} \mathrm{RS}\right)$. 


\subsection{The IR mapping of $\lambda \phi^{4}$ to Yang-Mills theories}

The pure gauge sector of QCD has been studied by Frasca by mapping it to the scalar self-interacting $\lambda \phi^{4}$ theory [384]. Frasca showed that in the IR limit or the classical limit, the mapping is complete. The scalar boson propagator is computed, and the running coupling can be deduced from it. The non-vanishing propagator leads to $\alpha\left(Q^{2}\right) \rightarrow 0$ as $Q^{2} \rightarrow 0$.

\subsection{The Bogoliubov compensation principle}

In Ref. [164], Arbuzov formed a nonperturbative coupling by applying the Bogoliubov compensation principle [385] to QCD. One uses SDE constraints to modify the one-loop pQCD expression of $\alpha_{s}$. The first-order approximate solution to this approach yields a gauge-invariant, RS-independent (1-loop expression) coupling which presents a finite maxima at the Landau pole position, and then vanishes in the deep IR region. The regulation of the Landau pole is due to a three-gluon interaction.

\subsection{Curci-Fermi model}

Tissier and collaborators have computed the gluon and ghost propagators, and the quark-gluon vertex with a 1-loop model including a gluon mass term [386]. They used the Curci-Ferrari model [387] as an effective description. The resulting coupling is finite, vanishes in the IR, and remains small enough to justify the use of perturbation theory in the IR domain. When converted to the $\overline{M S} \mathrm{RS}$, one finds $\alpha_{\overline{M S}} \lesssim 0.5$, except in the Landau gauge for which the coupling is significantly larger. The calculations were initially carried in the pure-gauge sector, with dynamical quarks subsequently introduced. 


\section{Chapter 5}

\section{Comparison and discussion}

\subsection{Validity of the comparison}

In this review we have discussed four distinct types of IR-behavior for the QCD running coupling, $\alpha_{s}\left(Q^{2}\right)$, using different theoretical frameworks: a divergent behavior, typically behaving as $1 / Q^{2}$; a freezing to an IR fixed point; a vanishing coupling $\alpha_{s}(0)=$ 0 ; or IR-finite behavior with nonzero slope. In fact, studies which agree qualitatively with one of these behaviors can still differ quantitatively. For example, the models which predict a freezing of $\alpha_{s}$ can disagree on its value by an order of magnitude. These qualitative and quantitative differences can have a number of causes.

We itemize below the causes that we have identified and illustrate them with examples.

Difference in the definitions In the perturbative domain, the coupling regulated in any RS with a massless gluon can be equivalently defined from the renormalization of any choice of the gluonic vertex: $\alpha_{s}^{g h}=\alpha_{s}^{3 g}=\alpha_{s}^{4 g}=\alpha_{s}^{q g}$. For example, in Section 3.5, the coupling was defined from the quark-gluon vertex $\alpha_{s}^{q g}$; see Eq. (3.38). However, in a RS with a massive gluon, the choice of definition ceases to be equivalent (Section 4.4.1). In addition, these couplings can display significant gauge-dependence at small $Q^{2}$.

Other definitions of the coupling employ an analytical expression based on pQCD, typically Eq. (3.17) and, in one way or another, supplement it with nonperturbative terms while retaining gauge invariance. The definitions can differ from each other since different nonperturbative contributions can be chosen. For example, a coupling defined as an effective charge comprises, by definition, all the nonperturbative contributions (including the observable-specific ones) thus making it observable-dependent. In contrast, 
nonperturbative contributions are only partially included in the analytic approach, but the coupling remains observable-independent.

We will discuss in more detail examples of adding nonperturbative terms in Section 5.3.

Differences due to the choice of the renormalization scheme The dependence of the QCD coupling on the choice of the RS can be studied in the UV by methods such as Commensurate Scale Relations. However, the dependence on scheme or effective charge can remain significant in the IR. This can explain the spread of freezing values seen in the literature $[162,388]$ as will be discussed in more detail in Section 5.2. The RS dependence has also been investigated in Refs. [183, 389, 390, 391]. In Ref. [392], couplings in the $\overline{M S}, \widehat{M O M}$ and the modified regularization invariant schemes were compared, with the conclusion that for these choices, the scheme-dependence is moderate. This is seemingly at odds with the results of Refs. $[162,388]$ for effective charges. The importance of the RS choice - or other arbitrary choices- on the IR-behavior of $\alpha_{s}$ clearly depends on the IR-definition of the coupling. For example, if the coupling is defined from an observable, the choice of gauge is irrelevant, whereas it is important for couplings defined from vertices.

Differences in the predicted value for $\Lambda$ within a given RS One expects that in a given scheme with the same value of $n_{f}$, the value of $\Lambda$ must be universal; In practice however, one finds a spread of values encountered in publications computing $\alpha_{s}$ in the IR.

Difference of relativistic forms The confining LF harmonic oscillator potential for light quarks in the front form - (Section 4.2) is equivalent to the nonrelativistic confining linear potential in the instant form (Section 4.3) [197]. Thus, even if one includes the same long-distance forces in the IR definition of $\alpha_{s}$, different relativistic forms and kinematic domains can lead to different analytic behavior of the running coupling; e.g., the Gaussian shape in the front form for light quarks and the $1 / Q^{2}$ behavior in the instant form for heavy static quarks. In fact, the exponential form for the coupling obtained from holographic QCD is only valid for light quarks, while the $1 / Q^{2}$ behavior is specific to heavy static quarks. 
Difference in gauge choices The effect of the gauge choice for gauge-dependent calculations of the coupling has been investigated by several authors [266, 393]. However, there is no consensus on the results. For example, studies using linear covariant gauges indicate that the gauge-dependence is weak for gauges chosen close to the Landau gauge $[223,311,312]$. In contrast, Aguilar and collaborators [227, 323] have argued that the differences between gauge-dependent and phenomenological (gauge-independent) calculations of $\alpha_{s}$ can be attributed to the choice of gauge. For example, in calculations yielding $\alpha_{s}(0) \simeq 3$ (see Section 5.4), the gluon propagator is typically computed in Landau gauge. In the phenomenological analyses, which tend to yield $\alpha_{s}(0) \simeq 0.6$, the Pinch Technique propagator (which is in effect similar to the Feynman gauge propagator) is relevant. The authors carry out Lattice QCD calculations in the two cases and recover the aforementioned differences. Likewise, the coupling defined using the Curci-Ferrari model [387] shows significant differences when computed in the Landau versus other gauges. The gauge dependence of $\alpha_{s}$ has also been discussed in Section 4.4.8.

Difference due to the choice of solution Two solutions can be found for couplings defined from vertices, (see Section 4.4.3), one leading to a finite non-zero freezing IR value of $\alpha_{s}$ (scaling solution), the other to an IR vanishing of $\alpha_{s}$ (decoupling solution). Some authors have focused on one or the other solution. For example, the authors in Ref. [266] have shown that the decoupling solution has a smaller value for the action than that of the scaling solution. This fact suggests why it is the decoupling solution that is more often found in lattice studies.

Difference in approximations Approximations are often necessary to make calculations of $\alpha_{s}$ tractable. Uncontrolled approximations will clearly produce different results. For example, in the case of the SDE (Section 4.4), integrating out the angles, as was done in early calculations, results in larger freezing values [242]. For example, in the pioneering analysis in Ref. [238], one finds that $\alpha_{s}^{g h}$ freezes at a value about 3 times higher than indicated by other determinations.

The coupling $\alpha_{s}^{g h}$ is particularly sensitive to the choice of approximations because it is defined as the product of $Z\left(Q^{2}, \mu\right)$ and $D\left(Q^{2}, \mu\right)$, the gluon and ghost propagators, respectively (see Eq. (4.25)). In the scaling solution, $Z\left(Q^{2}, \mu\right) \rightarrow 0$ and $D\left(Q^{2}, \mu\right) \rightarrow \infty$ in the IR. Thus, small differences in the approximations and the truncations employed, the choice of gauge, and even the level of numerical precision may lead to large differences in the freezing value. 
The reliability of SDE truncation schemes was studied and then improved in Refs. [394] and [260], reaching the conclusion that the standard scheme is reliable for qualitative estimates of the propagators and the IR coupling.

An important and still widely used approximation is to work in the pure gauge sector. To appreciate its effect, one can compare for example Eqs. (4.29) and (4.34) or look at Table 4.1 page 109. This approximation has been discussed within the SDE framework in Section 4.4.7. Unquenching the lattice calculations yields larger freezing values of $\alpha_{s}$ by a factor 2 , from 0.2 to 0.4 in e.g., Ref. [395], and in the static potential approach by $40 \%$, from $\alpha_{V} \simeq 0.3$ (quenched) to $\alpha_{V} \simeq 0.4\left(n_{f}=2+1\right)$ [206]. In the approach of Badalian et al. (BPT) in Refs. [44, 381], the $n_{f}=3$ IR freezing value increases by $\sim 20 \%$ compared to $n_{f}=1$ (here $\Lambda_{V}$ is fixed at $\sim 0.4 \mathrm{GeV}$ ). In [325], there is no direct influence from the quark loops, but they still alter the IR-behavior of $\alpha_{s}$ by affecting the value of the effective gluon mass. Other groups have argued that the influence of quarks is always small ([308, 342]).

In lattice QCD, the discretization and finite volume approximations may lead to unphysical artifacts, noticeably the IR-suppression of the value of $\alpha_{s}$. Furthermore, the choice of the mass of light quarks has been argued to be critical to the IR behavior of $\alpha_{s}[145]$.

Difference in pQCD order In several analyses, the pQCD expression for $\alpha_{s}$, Eq. (3.24), has been used to determine the IR-behavior; see Section 3.9.6, or to establish a modified expression for $\alpha_{s}$, see for example Eqs. (4.19), (4.28) or (4.54). In the last case, a low-order approximation is often used. As seen in Fig. 3.1, the magnitude of the coupling depends on the pQCD order. This dependence is controlled by the choice of parameters entering the IR expression for $\alpha_{s}$, e.g. the gluon effective mass, or similar scale parameters (see the enumeration page 59), the matching point between the IR and UV domain, and/or the value used for $\Lambda$.

Difference of the medium A physical argument given by Brodsky and Shrock for the origin of the freezing of $\alpha_{s}$ [6] suggests that such freezing within the color confinement domain of hadrons depends on the size of the host hadron: the larger the hadron, the smaller the $Q^{2}$-scale at which the freezing occurs (since it is due to the long wavelength cut-off above the characteristic size of the given hadron) and thus the larger the value of the frozen coupling, since the pQCD domain in which $\alpha_{p Q C D}$ grows with distance is larger. Furthermore, in the specific case of $\alpha_{V}$, some medium dependence 
is expected, in analogy to the Bethe state-dependent logarithm appearing in the Lamb Shift of hydrogenic atoms; see Section 3.7.1.

Medium dependence is also the expectation from the background perturbation theory (BPT); see Section 4.10. Other arguments have been put forth by Titard, Yndurain, and Pineda [396]. Finally, the inclusion of observable-dependent nonperturbative effects in the definition of the coupling, as is the case for effective charges (Sections 3.7.1 and 4.1), will naturally introduce medium dependence. However, this dependence is sometimes suppressed as illustrated by the effective charge $\alpha_{g_{1}}$ in Section 4.1.1.

Difference in group symmetries The strong coupling is sometimes computed in color $S U(2)$, where it has a larger value than in color $S U(3)$. In the pure gauge case, the comparison is straightforward: the coupling scales as $1 / N$. This comes from $\alpha_{p Q C D}$ factoring the IR-definitions of $\alpha_{s}$, see Eqs. (4.25), (4.41) and (4.42). For the pure gauge case, $\alpha_{p Q C D} \propto 1 / \beta_{0}=3 /(11 N)$. Thus, $3 \alpha_{s}^{S U(3)}=N \alpha_{s}^{S U(N)}$. This dependence is verified by Lattice calculations [329, 335], the stochastic quantization approach [237], and the FRG framework [337].

Difference in temperatures In this review, we have only considered calculations for zero temperature $T=0$.

Difference in space dimensionality Theoretical analyses of gauge couplings have been performed in 4D, 3D or 2D in Minkowski or Euclidean spaces. We have focused on the 4D calculations relevant to QCD, and have ignored a large body of work done for other numbers of dimensions, since the basic behavior of the coupling may be quite different. It is important to recall that only in $4 \mathrm{D}$ the QCD coupling is dimensionless.

Ignoring Gribov copies Choosing a particular solution among the Gribov copies and ignoring the others does not seem to affect the behavior of $\alpha_{s}$ : several groups using different approaches have concluded that Gribov copies have little or no effect, see Section 4.7.

The above list demonstrates that the different predictions for the QCD coupling in the IR cannot be compared straightforwardly. Correcting for these differences to obtain a valid comparison of published results goes well beyond the scope of this review. However, one can attempt to roughly assess the effects of these differences to ascertain 


\begin{tabular}{|c|c|c|c|}
\hline$\alpha_{s}(0)$ & $\mathrm{RS}$ & $Q_{0}^{2}(\mathrm{GeV})$ & $\Lambda(\mathrm{GeV})$ \\
\hline \hline $1.22 \pm 0.04 \pm 0.11 \pm 0.09$ & $\overline{M S}$ & $0.75 \pm 0.03 \pm 0.05 \pm 0.04$ & $0.34 \pm 0.02$ \\
\hline $2.30 \pm 0.03 \pm 0.28 \pm 0.21$ & $V$ & $1.00 \pm 0.00 \pm 0.07 \pm 0.06$ & $0.37 \pm 0.02$ \\
\hline $3.79 \pm 0.06 \pm 0.65 \pm 0.46$ & $M O M(\mathrm{~L})$ & $1.32 \pm 0.02 \pm 0.10 \pm 0.08$ & $0.52 \pm 0.03$ \\
\hline $3.51 \pm 0.14 \pm 0.49 \pm 0.35$ & $g_{1}$ & $1.14 \pm 0.04 \pm 0.08 \pm 0.06$ & $0.92 \pm 0.05$ \\
\hline
\end{tabular}

Table 5.1: Column 1: values of $\alpha_{s}(0)$ calculated in different RS (column 2). The MOM results are in the Landau gauge. The transition scale $Q_{0}^{2}$ is given in column 3. Column 4 gives the input values used for $\Lambda$ for each RS. The uncertainties on $\alpha_{s}(0)$ and $Q_{0}^{2}$ comes (from left to right) from the uncertainty on $\alpha_{p Q C D}$, the uncertainty on the hadronic scale $\kappa$ in LFHQCD, and an assigned $5 \%$ uncertainty for $\Lambda$. The table is from Ref. [388]

if a consensus on the IR-behavior of $\alpha_{s}$ could be in sight. First, we will discuss the fact that couplings expressed in different RS must freeze at different values, and how to account for this difference.

\subsection{Influence of the renormalization scheme}

The influence of RS can be quantitatively assessed using the light front holographic QCD approach [388]. In Section 3.9.6, $\alpha_{p Q C D}$ in a given RS is matched to $\alpha_{A d S}$ to determine the perturbative QCD scale $\Lambda$ and the matching point $Q_{0}$, which in turn determines the quark-hadron transition. Rather than determining $\Lambda$, one can use the known value of $\Lambda$ and leave the RS-dependent freezing value $\alpha_{s}(0)$ as a free parameter, together with $Q_{0}$. This allows the IR-behavior of $\alpha_{s}$ to be established in any RS, see Fig. 5.1. The different freezing values obtained are given in Table 5.1.

The $\overline{M S}$ freezing value obtained from the matching procedure is $\alpha_{\overline{M S}}(0)=1.22 \pm$ 0.15, twice as large as than the result obtained from phenomenology (i.e. mainly from the spectroscopic approach, see tables 5.2 and 5.3) typically $0.5 \pm 0.2$. It is closer to Cornwall's $\overline{M S}$ results including quarks $\left(\alpha_{\overline{M S}}(0)=0.81\right.$, Section 4.4.7), and comparable to the results using Shirkov's analytical coupling $\left(\alpha_{\overline{M S}}(0)=1.25\right.$, Section 4.9), and those of Gracey's $\overline{M S}$ calculation using the Gribov-Zwanziger approach $\left(\alpha_{\overline{M S}}(0)=1.70\right.$, Section 4.7). The value of $Q_{0}$ is consistent with that found by Gomez and Natale [397]. However, a value of $Q_{0}$ that is smaller than $1 \mathrm{GeV}$ is inconsistent with the analysis of Badalian and collaborators of the charmonium and bottomonium fine-structure data [398]. Their coupling at $Q^{2}=1 \mathrm{GeV}^{2}, \alpha_{\overline{M S}}\left(Q^{2}=1\right) \simeq 0.4$, is already significantly influenced by IR effects: it is about $50 \%$ lower than the pQCD prediction. However, the analysis of Badalian and collaborators is done at 2-loops, whereas the results reported in Table 5.1 


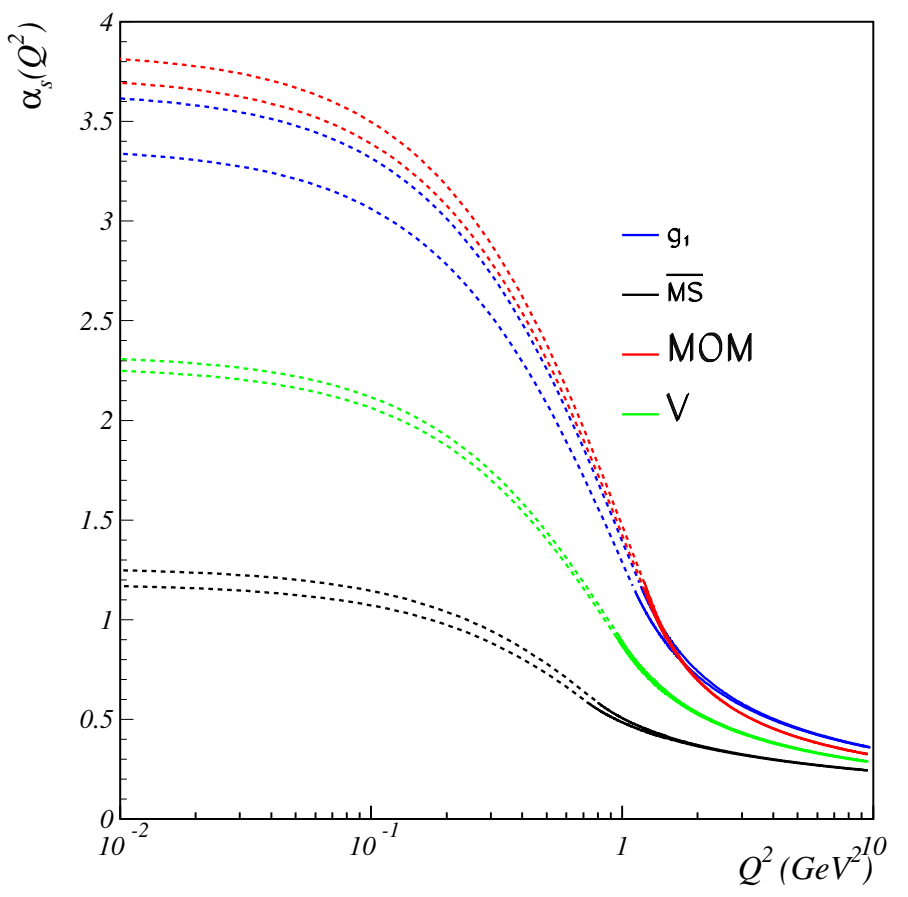

Figure 5.1: How different renormalization schemes lead to different freezing values for $\alpha_{s}$. The black dashed line represents the AdS/QCD continuation of $\alpha_{p Q C D}$ in the $\overline{M S}$ scheme (continuous black line), the blue line is the effective charge $\alpha_{g_{1}}$ in the $g_{1}$ scheme (without enforcing the $\alpha_{g_{1}}(0)=\pi$ constraint), the green line is the effective charge $\alpha_{V}$ in the potential scheme and the red line is $\alpha_{s}$ in the MOM scheme and Landau gauge. The widths of the curves represent the uncertainty stemming from the truncation of the $\mathrm{pQCD} \beta$-series.

are obtained at 4-loops. The $2.84 \pm 0.02$ freezing value in the MOM scheme and Landau gauge is in close agreement with numerous $\alpha_{s}^{g h} \simeq 3$ results obtained in the MOM scheme and Landau gauge using SDE and lattice gauge theory.

This approximate agreement indicates that different choices of scheme may explain a significant part of the spread of freezing values seen in the literature. However, as discussed at the beginning of this section, other factors must be considered before a satisfactory comparison is made, the main factor being the particular IR-definition of $\alpha_{s}$. For example, correcting for the RS does not explain the disagreement between the effective charges extracted from the Bjorken sum and from $\tau$-decay: The coupling $\alpha_{\tau}(s)$ extracted from experimental data [84] in Ref. [39] freezes around $\alpha_{\tau}(0) \simeq 7$. Correcting to the $g_{1}$ scheme, the freezing value becomes $\alpha_{(\tau) g_{1}}(0) \simeq 6$, roughly twice the expected value of $\pi$. This discrepancy may be due to the unsubtracted pion pole that is part of $\alpha_{\tau}(s)$, which by analogy, is analogous to the elastic contribution that must be removed from $\alpha_{g_{1}}$ (see Section 4.1). 


\subsection{The contributions of nonperturbative terms to $\alpha_{s}\left(Q^{2}\right)$}

The QCD running coupling $\alpha_{s}\left(Q^{2}\right)$ invariably includes dynamics associated with nonperturbative physics. This is particularly clear when one defines the coupling in terms of an effective charge defined from a physical observable. In addition, the coupling at large momentum transfer will also depend on the choice of the renormalization scheme. The reasons behind the RS-dependence of $\alpha_{s}\left(Q^{2}\right)$ are not manifest in our previous discussion. They can be better apprehended if the explanation is recast in terms of non-perturbative effects. In this section we shall show, using two examples, how highertwist terms remove the artificial pQCD divergence (Landau pole) and how they shape the behavior of $\alpha_{s}\left(Q^{2}\right)$ at low $Q^{2}$. We will see in particular how the transition between the IR behaviors of $\alpha_{g_{1}}\left(g_{1}\right.$ scheme $)$ and $\alpha_{\overline{M S}}(\overline{M S}$ scheme) occurs.

The role of nonperturbative contributions and the renormalization scheme dependence can be illustrated using the effective charge $\alpha_{g_{1}}$ [399]. The integral entering the Bjorken sum rule $\int_{0}^{1} d x_{f}\left[g_{1}^{p}\left(x_{f}, Q^{2}\right)-g_{1}^{n}\left(x_{f}, Q^{2}\right)\right]$ can be expanded in powers of $1 / Q^{2}$ using guidance from the $O P E$ :

$$
\int_{0}^{1} d x_{f}\left(g_{1}^{p}\left(x_{f}, Q^{2}\right)-g_{1}^{n}\left(x_{f}, Q^{2}\right)\right)=\sum_{i=1}^{\infty} \frac{\mu_{2 i}}{Q^{2 i-2}}
$$

The integration includes the $x_{f}=1$ elastic contribution which, by definition, is highertwist. The leading-twist term is given by Eq. (3.40). We recall it here and adopt the estimate for the $\alpha \frac{5}{M S}$ coefficient given by Kataev [400]:

$$
\begin{aligned}
\mu_{2}\left(Q^{2}\right)=\frac{g_{A}}{6}\left[1-\frac{\alpha_{\overline{M S}}}{\pi}-3.58\left(\frac{\alpha_{\overline{M S}}}{\pi}\right)^{2}-20.21\left(\frac{\alpha_{\overline{M S}}}{\pi}\right)^{3}\right. \\
\left.-175.7\left(\frac{\alpha_{\overline{M S}}}{\pi}\right)^{4}-893.38\left(\frac{\alpha_{\overline{M S}}}{\pi}\right)^{5}+\mathcal{O}\left(\alpha_{\overline{M S}}^{6}\right)\right] .
\end{aligned}
$$

From Eqs. (4.1), (5.1) and (5.2), one has:

$$
\begin{aligned}
\frac{\alpha_{g_{1}}\left(Q^{2}\right)}{\pi}=\frac{\alpha_{\overline{M S}}}{\pi} & +3.58\left(\frac{\alpha_{\overline{M S}}}{\pi}\right)^{2}+20.21\left(\frac{\alpha_{\overline{M S}}}{\pi}\right)^{3} \\
& +175.7\left(\frac{\alpha_{\overline{M S}}}{\pi}\right)^{4}+893.38\left(\frac{\alpha_{\overline{M S}}}{\pi}\right)^{5}+\mathcal{O}\left(\alpha_{\overline{M S}}^{6}\right)-\frac{6}{g_{A}} \sum_{i=2} \frac{\mu_{2 i}}{Q^{2 i-2}}
\end{aligned}
$$


The nonperturbative terms are represented by the $1 / Q^{2 i-2}, i>2$, power corrections; i.e., higher-twist contributions of order $2 i$. Each higher-twist coefficient $\mu_{i}$ can be expressed as a sum of kinematical twist terms with a power smaller than $2 i$ as well as a dynamical term of order $2 i$. For example, the twist-4 coefficient is [401]:

$$
\mu_{4}=\frac{M^{2}}{9}\left(a_{2}^{p-n}+4 d_{2}^{p-n}+4 f_{2}^{p-n}\right)
$$

where $a_{2}^{p-n}$ is the twist-2 target-mass correction given by the $x_{f}^{2}$-weighted moment of the leading-twist $g_{1}^{p-n, L T}$ structure function: $a_{2}^{p-n}=\int_{0}^{1} d x_{f}\left(x_{f}^{2} g_{1}^{p-n}\right)$. The twist-3 matrix element $d_{2}^{p-n}$ is given by:

$$
d_{2}^{L T}=\int_{0}^{1} d x_{f} x_{f}^{2}\left(2 g_{1}^{p-n, L T}+3 g_{2}^{p-n, L T}\right)
$$

where the spin structure function $g_{2}$ provides the twist-3 contribution. The function $f_{2}^{p-n}$ is the pure twist-4 contribution. The dynamical higher-twist terms correspond physically to the interactions between the struck quark and the nucleon's quark spectators, which by definition, are nonperturbative contributions associated with the bound-state dynamics. The coefficients are modified by powers of $\log \left(Q^{2}\right)$ due to DGLAP evolution.

The result of unfolding the twist-4 and the higher order pQCD corrections to $\mu_{2}$ in $\alpha_{g_{1}}$ is illustrated in Fig. 5.2. The blue squares give the effective charge $\alpha_{g_{1}}\left(Q^{2}\right)$, Eq. (3.41), as shown in Fig. 4.1. The magenta open circles show $\alpha_{g_{1}}^{\neg \mathrm{RC}}$, that is the coupling obtained after unfolding from $\alpha_{g_{1}}$ the $\mu_{2}$ pQCD radiative corrections (RC) up to $\mathrm{N}^{4} \mathrm{LO}$. The black stars are for $\alpha_{g_{1}}^{\neg \text { RC, HT }}$ when the twist-4 term is excluded from $\alpha_{g_{1}}$ as well. In practice the three sets of data shown in Fig. 5.2 are obtained from solving three different equations that contains or not the pQCD soft radiation effects and higher-twists: The blue squares are obtain from solving Eq. (3.41); The magenta circles are obtained from solving Eq. (3.40), or equivalently from solving Eq. (5.1) for $i_{\max }=1$; The black stars are obtained from solving Eq. (5.1) for $i_{\max }=2$.

To exclude the twist- 4 term, we use the following procedure: A fit to the polarized parton distributions [402] can be used to determine the twist-2 $a_{2}^{p-n}$ term in Eq. 5.4: At $Q^{2}=1 \mathrm{GeV}^{2}, a_{2}^{p-n}=0.031$. The $d_{2}^{p-n}$ matrix element is obtained from Refs. [176] and [403] and evolved from $Q^{2}=5 \mathrm{GeV}^{2}$ to $1 \mathrm{GeV}^{2}$. This yields $d_{2}^{p-n}=-0.008$. The pure twist-4 term $\alpha_{\overline{M S}}\left(Q^{2}\right)$ is known experimentally $[70,177]$ based on the use of the $\alpha_{\overline{M S}}\left(Q^{2}\right)$ scheme. However, for a proper investigation of the role of higher-twist contributions in $\alpha_{g_{1}}\left(Q^{2}\right)$, the value of $f_{2}^{p-n}$ must be obtained independently of $\alpha_{\overline{M S}}\left(Q^{2}\right)$. 


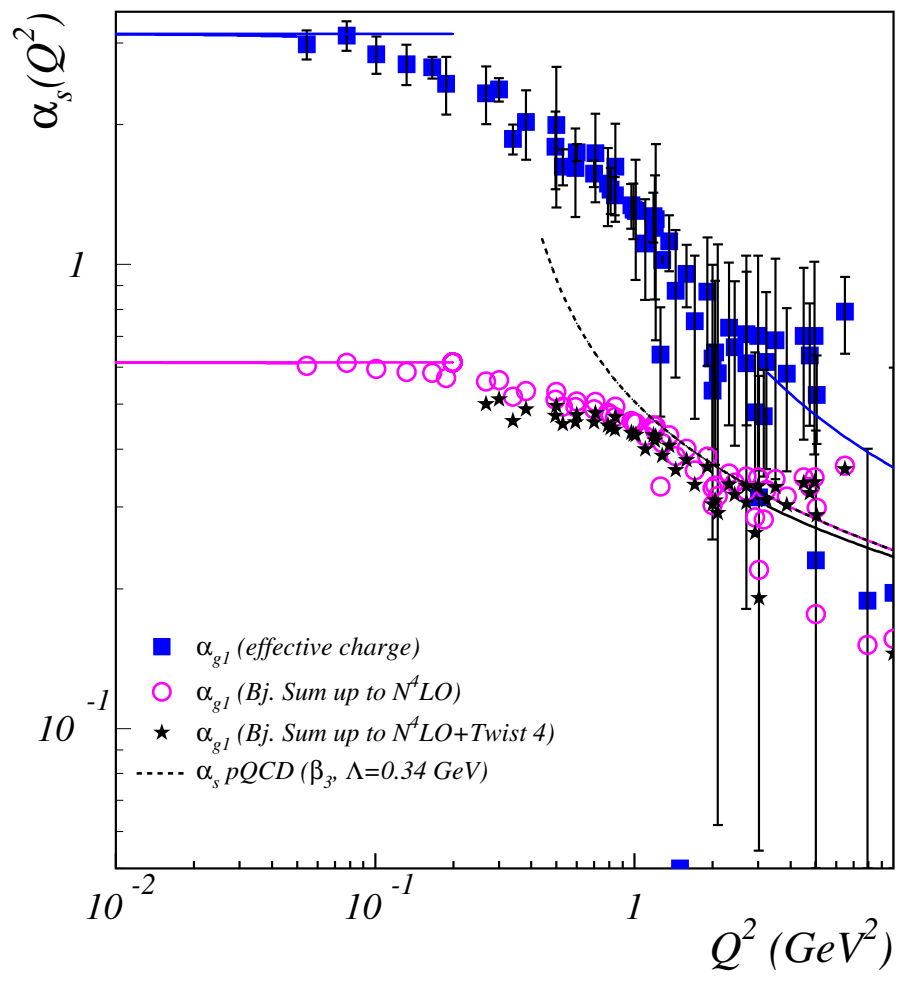

Figure 5.2: Experimental data and sum rule constraints for the effective charges $\alpha_{g_{1}}\left(Q^{2}\right)$ and $\alpha_{F_{3}}\left(Q^{2}\right)$ (blue squares). The magenta circles show the effect of unfolding from $\alpha_{g_{1}}$ the $\mathrm{N}^{4} \mathrm{LO}$ pQCD corrections to $\mu_{2}$. The black stars correspond to the case when the higher-twist coefficient $\mu_{4}$ is unfolded as well. The continuous lines of matching colors are the corresponding predictions from the GDH (low- $Q^{2}$ domain) and Bjorken (high- $Q^{2}$ domain) sum rules. The dashed black line is the pQCD expectation calculated up to $\beta_{3}$. For clarity, we show the uncertainties only for the blue square data. 
(If one uses the experimentally extracted value of $f_{2}^{p-n}$, one would have by construction $\left.\alpha_{g_{1}}^{\neg \mathrm{RC}, \mathrm{HT}}\left(Q^{2}\right)=\alpha_{\overline{M S}}\left(Q^{2}\right)\right)$. Theoretical calculations of $f_{2}$ do exist [404] giving $f_{2}^{p-n}=-0.03$ after subtracting an elastic contribution to $f_{2}$ of -0.02 . The power corrections reintroduce divergent $1 / Q^{2 n}$ terms. It is normally acceptable to use the $O P E$ formalism down to $Q^{2} \sim 1 \mathrm{GeV}^{2}$. In fact, the higher-twist series may still converge at lower $Q^{2}$ : For example, at $Q^{2}=0.5 \mathrm{GeV}^{2}$ the higher-twist coefficient $\mu_{4}^{2} / Q^{2}=0.3$ is well below unity.

The continuous lines in Fig. 5.2 are the predictions from the GDH sum rule at low$Q^{2}$, Eqs. (4.3) and (4.4), and from the Bjorken sum rule at high- $Q^{2}$, Eq. (4.1). Once the pQCD and higher-twist corrections are unfolded from $\alpha_{g_{1}}$, the coupling $\alpha_{g_{1}}$ RC, HT agrees well at large $Q^{2}$ with the pQCD expectation given by the dashed line. At low $Q^{2}$, the freezing value of $\alpha_{g_{1}}^{\neg \mathrm{RC}, \mathrm{HT}}$ is now about 0.6 , closer to the value found in many $\overline{M S}$ determinations of $\alpha_{s}$; see the list in the next Section and see also Fig. 5.3.

We note that accounting for the twist-4 contributions lower the freezing value from 0.62 to about 0.5 . This is because $\mu_{4}<0$. Experimental studies indicate that $\mu_{6} \sim 0$ and $\mu_{8} \sim-\mu_{4}>0[70,177]$. Consequently, the addition of more higher-twist contributions would increase the freezing value, possibly close to the 1.2 value seen in Fig. 5.1. In addition to revealing the mechanisms behind the RS-dependence of $\alpha_{s}(0)$, namely here how one makes the transition between $\alpha_{g_{1}}$ and $\alpha_{\overline{M S}}$, this analysis also shows that the failure to account for nonperturbative effects, behaving as $1 / Q^{n}$ at intermediate $Q^{2}$, can lead to a Landau pole.

As an alternative to effective charges, we can use Shirkov's analytic coupling to study the effect of higher-twists. We recall here its LO form:

$$
\alpha_{a n}^{(0)}\left(Q^{2}\right)=\frac{4 \pi}{\beta_{0}}\left(\frac{1}{\ln \left(Q^{2} / \Lambda^{2}\right)}+\frac{\Lambda^{2}}{\Lambda^{2}-Q^{2}}\right) .
$$

For $Q^{2} \gg \Lambda^{2}$, the domain where $O P E$ is valid, the contributions beyond the leadingpower pQCD contributions can be expanded using $\Lambda^{2} /\left(\Lambda^{2}-Q^{2}\right)=-\sum_{n=1}\left(\Lambda^{2} / Q^{2}\right)^{n}$, in apparent agreement with the form of the power corrections expected from the OPE. We note the negative sign which compensates for the positively diverging Landau pole. Taking the $O P E$ expansion of some observable:

$$
A\left(Q^{2}\right)=\sum_{n=0} a_{n} \alpha_{p Q C D}^{n}\left(Q^{2}\right)+\sum_{i=1} \frac{b_{i}\left(Q^{2}\right)}{Q^{2 i}}
$$


and expressing it using Eq. (5.6) i.e., with power corrections folded in the coupling:

$$
A\left(Q^{2}\right)=\sum_{n=0} a_{n}^{\prime} \alpha_{a n}^{n}\left(Q^{2}\right)
$$

one has $a_{n}=a_{n}^{\prime}, b_{1}=-\Lambda^{2}\left(a_{1}+2 a_{2} \alpha_{p Q C D}+\cdots\right) 4 \pi / \beta_{0}, b_{2}=-\Lambda^{4}\left(a_{1}+a_{2}\left(2 \alpha_{p Q C D}-\right.\right.$ $\left.4 \pi / \beta_{0}+\cdots\right) 4 \pi / \beta_{0}, \cdots$. The results obtained for the $b_{i}$ are observable-dependent (through the coefficients $a_{n}$ ) and have a $Q^{2}$ logarithmic dependence in the UV (through $\left.\alpha_{p Q C D}\right)$, as expected. However, the $Q^{2}$-dependent functions $b_{i}$ do not include all nonperturbative effects. For example, $a_{n}$ and $\alpha_{p Q C D}$ include only short-distance phenomena (gluon bremsstrahlung, vacuum polarization) whereas some of the higher-twist contributions represent the transverse, long-distance force responsible for confinement [167]. Nevertheless, using $\alpha_{a n}$, rather than $\alpha_{p Q C D}$, identifies in (5.7) a well-defined nonperturbative contribution which should increase the validity range of $O P E$ down to values of $Q^{2}$ close to $\Lambda^{2}$. Such an approach is discussed in more depth in Ref. [405].

\subsection{Listing of the multiple IR-behavior found in the literature}

In order to facilitate the comparison between the multiple determinations which describe the IR-behavior of $\alpha_{s}$ cited in this review, we summarize the main results in Tables 5.2 and 5.3. The corresponding predictions for $\alpha_{s}(0)$ are also plotted in Fig. 5.3, where vertical arrows indicate divergent couplings. (We do not include in Fig. 5.3 the results from couplings defined from the 3 -gluon and 4-gluon vertices). This figure does not correct for differences in RS, gauges or definitions. Consequently, we are not necessarily comparing the same quantities. Furthermore, we have plotted some pioneering results, such as the first SDE determination of $\alpha_{s}^{g h}$ by von Smekal et. al. [238], now superseded by more accurate calculations.

Four main groups of results can be identified:

- $\alpha_{s}\left(Q^{2}\right) \overrightarrow{Q^{2} \rightarrow 0} \infty$ : static $Q-\bar{Q}$ potential with the string tension folded in $\alpha_{s}$, and various nonperturbative models.

- $\alpha_{s}\left(Q^{2}\right) \sim 3: \alpha_{s}^{g h}(0)$ for the scaling solution (SDE, lattice, FRG, stochastic quantization), and the effective charges $\alpha_{g_{1}}(0)$ and $\alpha_{F 3}(0)$. 


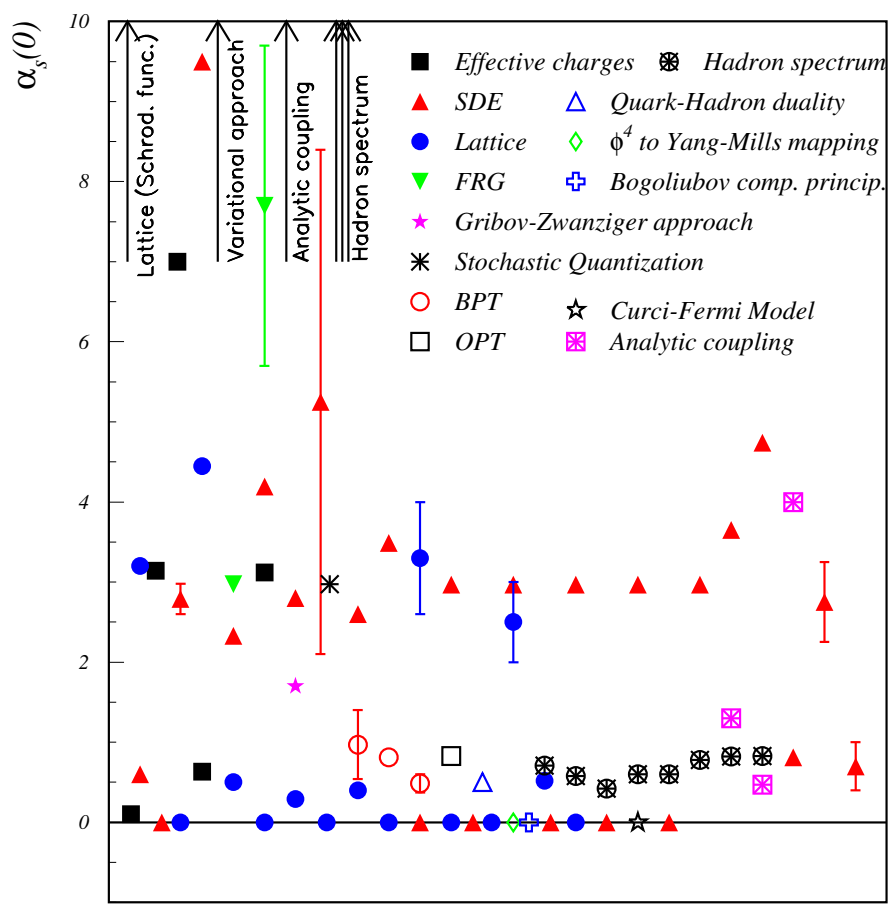

Figure 5.3: Values of $\alpha_{s}(0)$ from the literature and reported in this review. The differences in the infrared fixed-point value of the coupling can be due to choices of RS, gauge, relativistic form, truncations, approximations, model dependence and other points, as indicated and discussed in the main text. The vertical arrows indicate IR-divergent couplings. 
- $\alpha_{s}\left(Q^{2}\right) \sim 0.7$ : quark models and the static $Q-\bar{Q}$ potential without the string tension folded in $\alpha_{s}$.

- $\alpha_{s}\left(Q^{2}\right)=0$ : decoupling solution (SDE, lattice, FRG) and various nonperturbative models.

We do not attempt to reconcile all the results, but the preceding discussion suggests that most can be made consistent, at least qualitatively.

Reconciling the $\alpha_{s}(0) \sim 0.7$ group with the $\alpha_{s}(0) \sim 3$ group In Ref. [323], the difference between the group $\alpha_{s}(0) \sim 0.7$ and the gauge-dependent results pertaining to the $\alpha_{s}(0) \sim 3$ group is attributed to different gauge choices: it is argued that the Feynman gauge produces results close to the phenomenological value $\alpha_{s}(0) \sim 0.7$, whereas the Landau gauge yields $\alpha_{s}(0) \sim 3$. Furthermore, it was shown in Ref. [162], and discussed in the beginning of this Section, that the other gauge-independent results belonging to this group computed in the $g_{1}$ or $\overline{M S} \mathrm{RS}$ agree with the results computed in the $M O M$ RS once the RS-dependence is corrected for, see Fig. 5.1. The AdS/QCD continuation of the pQCD calculation yields a higher $\alpha_{g_{1}}(0)$ than expected, but is still compatible within uncertainties with the $\pi$ constraint. Similarly, the freezing value for $\alpha_{\overline{M S}}$ obtained from the AdS/QCD-pQCD matching has a larger central value than the typical 0.7 freezing value.

Reconciling the divergent $\alpha_{s}\left(Q^{2}\right) \overrightarrow{Q^{2} \rightarrow 0} \infty$ group with the $\alpha_{s}(0) \simeq 0.7$ and $\alpha_{s}(0) \simeq$ 3 groups As we have emphasized, the QCD running coupling does not need to be divergent in the IR in order for a theory to be confining. For example, the coupling for the analytically solvable [406] confining theory $\mathrm{QCD}(1+1)$ is finite.

Confinement in QCD can be due to multiple gluon exchange diagrams, where the exchanged gluons are connected by the gluon and four-gluon couplings as in the " $\mathrm{H}$ " diagrams; see Fig. 2.2d. The network of gluons corresponds to a "string" or "flux tube" connecting the $Q$ and $\overline{\mathrm{Q}}$. The fact that the $\mathrm{H}$ diagram becomes IR divergent as the $Q$ and $\overline{\mathrm{Q}}$ separate in impact space is indicative that $\mathrm{QCD}$ must confine color charges in order to render the theory self-consistent. Thus the infinite sum of such diagrams could be the source of the confining potential. For example, the confining harmonic oscillator potential between light quarks and antiquarks in the LF Hamiltonian derived using light-front holography and AdS/QCD may well come from the IR behavior of the sum of $\mathrm{H}$ graphs, and has been shown to be equivalent to the diverging linear potential 


\begin{tabular}{|c|c|c|c|c|c|c|c|}
\hline$\alpha_{s}(0)$ & IR behavior & Definition & $\mathrm{RS}$ & Gauge & Quarks & Framework & References \\
\hline 0.10 & mono. decr. & Sudakov eff. charge & $V$ & Indep. & yes & Sudakov resum. & {$[183,184]$} \\
\hline$\pi$ & freezes & eff. charge & $g_{1}$ & Indep. & yes & Measurements & {$[182]$} \\
\hline 3.12 & freezes & eff. charge & $g_{1}$ & Indep. & yes & AdS/QCD & {$[162,125]$} \\
\hline$\sim 7$ & freezes & eff. charge & $\tau$ & Indep. & yes & Measurements & {$[39]$} \\
\hline 0.63 & freezes & dressed gl. exch. & $\sim V$ & Indep. & yes & Disp. approach & {$[168,169]$} \\
\hline 0.60 & freezes & gl. self-energy & $(\sim \overline{M S})$ & Indep. & no & $\operatorname{SDE} \Lambda=0.26$ & {$[281]$} \\
\hline 0 & vanishes & gl. self-energy & $(\overline{M S}, \mathrm{MOM})$ & Landau & no & SDE $0.3 \leq \Lambda \leq 0.8$ & {$[259]$} \\
\hline $0.7 \pm 0.3$ & freezes & gl. self-e., gh.-gl. vert. & MOM & Landau & no & $\operatorname{SDE} \Lambda=0.3$ & {$[227]$} \\
\hline- & freezes & gl. self-energy & $(\sim \overline{M S})$ & Feynman & no & SDE $\Lambda=0.3$ & {$[233]$} \\
\hline $2.6-2.97$ & freezes & gh.-gl. vertex & $\mathrm{MOM}$ & Landau & no & SDE & {$[252]$} \\
\hline- & freezes & 3- and 4-gl. vertex & MOM & Landau & no & $\mathrm{SDE}$ & {$[300]$} \\
\hline Diverges & $1 / Q^{2}$ & quark-gl. vertex & $\mathrm{MOM}$ & Landau & no & $\mathrm{SDE}$ & {$[309]$} \\
\hline$\sim 2.5$ & freezes & quark-gl. vertex & $\mathrm{MOM}$ & Landau & yes & $\mathrm{SDE}$ & {$[309]$} \\
\hline 2.33 & freezes & gh.-gl. vertex & - & Coulomb & no & $\mathrm{SDE}$ & {$[312]$} \\
\hline 4.19 & freezes & gh.-gl. vertex & $(\sim \overline{M S})$ & Landau & no & SDE $\Lambda=0.153$ & {$[239]$} \\
\hline$\sim 2.8$ & freezes & Int. strength & $\overline{M S}$ & Indep. & no & SDE+Lattice & {$[285]$} \\
\hline 2.1 to 8.4 & freezes & gh.-gl. vertex & $\overline{M S} ?$ & Landau & no & $\mathrm{SDE}$ & {$[240]$} \\
\hline 2.6 & freezes & gh.-gl. vertex & $\overline{M S}$ & Landau & yes & $\mathrm{SDE}$ & [296] \\
\hline 3.49 & freezes & gh.-gl. vertex & $\left(g_{1}\right)$ & Landau & no & SDE $\Lambda=0.856$ & {$[245]$} \\
\hline 0 & vanishes & gh.-gl., 3-gl. vert. & $\mathrm{MOM}$ & Landau & no & SDE & {$[303]$} \\
\hline 0.81 & freezes & gl. self-energy & $(\sim \overline{M S})$ & Light cone & yes & $\operatorname{SDE} \Lambda=0.26$ & {$[226,289]$} \\
\hline 2.97 & freezes & gh.-gl., & $\mathrm{MOM}$ & Landau & no & $\mathrm{SDE}$ & {$[307]$} \\
\hline $3.2 \times 10^{-3}$ & freezes & 3-gl. vertex & $\mathrm{MOM}$ & Landau & no & $\mathrm{SDE}$ & {$[307]$} \\
\hline $4.2 \times 10^{-2}$ & freezes & 4-gl. vertex & $\mathrm{MOM}$ & Landau & no & $\mathrm{SDE}$ & {$[307]$} \\
\hline 0 & vanishes & gh.-gl., 3-gl., 4-gl. & MOM & Landau & no & $\mathrm{SDE}$ & {$[307]$} \\
\hline 2.97 & freezes & gh.-gl. vertex & MOM & Landau & no & $\mathrm{SDE}$ & {$[302]$} \\
\hline 0 & vanishes & gh.-gl. vertex & $\mathrm{MOM}$ & Landau & no & SDE & {$[302]$} \\
\hline $1.6 \times 10^{-3}$ & freezes & 3-gl. vertex & MOM & Landau & no & SDE & {$[302]$} \\
\hline 0 & vanishes & 3-gl. vertex & $\mathrm{MOM}$ & Landau & no & $\mathrm{SDE}$ & {$[302]$} \\
\hline 2.97 & freezes & gh.-gl. vertex & MOM & Landau & no & $\mathrm{SDE}$ & {$[242]$} \\
\hline 0 & vanishes & gh.-gl., 3-gl. & $\mathrm{MOM}$ & Lin. covar. & no & $\mathrm{SDE}$ & {$[313]$} \\
\hline 2.97 & freezes & gh.-gl. & $\mathrm{MOM}$ & Landau & no & $\mathrm{SDE}$ & {$[306]$} \\
\hline $2.77 \times 10^{-3}$ & freezes & 4-gl. vertex & $\mathrm{MOM}$ & Landau & no & $\mathrm{SDE}$ & {$[306]$} \\
\hline 3.65 & freezes & gh.-gl. vertex & - & Landau & no & $\mathrm{SDE}$ & {$[235]$} \\
\hline 4.74 & freezes & gh.-gl. vertex & - & Coulomb & no & $\mathrm{SDE}$ & {$[235]$} \\
\hline 9.5 & freezes & gh.-gl. vertex & $\mathrm{MOM}$ & Landau & no & SDE & {$[238]$} \\
\hline
\end{tabular}

Table 5.2: Summary of the predictions for the IR behavior of $\alpha_{s}\left(Q^{2}\right)$ discussed in this review. As emphasized in the text, the results depend on the different definitions of the running coupling. The label "indep." denotes predictions which are gauge- or RS-independent. The expressions for $\alpha_{s}$ are formally scheme-independent when expressed at LO or NLO, but they remain numerically dependent on the scheme choice through the value used for $\Lambda$. To convey this dependence, we have identified within parenthesis the choice of scheme corresponding to the $\Lambda$ value given in the $7^{\text {th }}$ column. The sign "-" indicates that information is either unclear or unknown. All results are for $S U(3)_{C}$ in 3+1-dimensional space-time. 


\begin{tabular}{|c|c|c|c|c|c|c|c|}
\hline$\alpha_{s}(0)$ & IR behavior & Definition & $\mathrm{RS}$ & Gauge & Quarks & Framework & References \\
\hline 3.2 & freezes & gh.-gl. vertex & MOM & Landau & yes & Lattice & [325] \\
\hline 4.45 & freezes & gh.-gl. vertex & MOM & Landau & no & Lattice & [323] \\
\hline 0.5 & freezes & pQCD form & MOM & Indep. & no & Lattice & [323] \\
\hline $0.29 \pm 0.03$ & freezes & Q-Q pot. & $V$ & Indep. & no & Lattice & {$[206]$} \\
\hline 0.40 & freezes & Q-Q pot. & $V$ & Indep. & yes & Lattice & {$[206]$} \\
\hline $3.3 \pm 0.7$ & freezes & gh.-gl. vertex & $(\tau)$ & Landau & no & Lattice $\Lambda=1.1$ & {$[230]$} \\
\hline 0 & vanishes & gh.-gl. vertex & MOM & Landau & no & Lattice & see caption \\
\hline 0 & vanishes & gh.-gl. vertex & MOM & Landau & yes & Lattice & {$[257,270]$} \\
\hline $2.5 \pm 0.5$ & freezes & gh.-gl., q.-gl. v. & MOM & Land. Coul. & yes & Lattice & [251] \\
\hline 0.52 & freezes & Q-Q pot. & $V$ & Indep. & yes & Lat. pert. stoch. th. & {$[332]$} \\
\hline diverges & $e^{m / Q}$ & Schrod. func. & $\overline{M S}$ & - & yes & Lattice & {$[335]$} \\
\hline 2.97 & freezes & gh.-gl. vertex & - & indep. & yes & Stoch. Quant. & {$[237,342]$} \\
\hline$\infty$ & $1 / Q^{2}$ & pQCD form & $V$ & Coulomb & yes & Variational ap. & {$[157]$} \\
\hline 0.71 & freezes & pQCD form & $\overline{M S}$ & Indep. & yes & Pheno. & {$[216]$} \\
\hline$\infty$ & $1 / Q^{2}$ & pQCD form & $V$ & Indep. & yes & Pheno. & [288] \\
\hline 0.58 & freezes & pQCD form & V & Indep. & yes & Pheno. & [215], \\
\hline$\infty$ & $1 / Q^{2}$ & pQCD form & $\overline{M S}$ & Indep. & yes & Pheno. & {$[220]$} \\
\hline $0.42 \pm 0.03$ & (Av. IR val.) & $\mathrm{pQCD}$ form & - & Indep. & yes & Pheno. & {$[200]$} \\
\hline$\simeq 0.6$ & freezes & pQCD form & - & Indep. & yes & Pheno. & [200] \\
\hline 0.78 & mono. incr. & pQCD form & $\overline{M S}$ & Indep. & yes & Pheno. & {$[368]$} \\
\hline 0.60 & freezes & pQCD form & $(\overline{M S})$ & Indep. & yes & Pheno. $\Lambda=0.2$ & {$[213]$} \\
\hline 0.82 & freezes & pQCD form & $(\overline{M S})$ & Indep. & yes & Pheno. & {$[286]$} \\
\hline diverges & $1 / Q^{2}$ & pQCD form & $V$ & Indep. & yes & Pheno. & [218] \\
\hline 0.83 & freezes & $\mathrm{pQCD}$ form & - & Indep. & yes & Pheno. & {$[214]$} \\
\hline 0.83 & freezes & $\alpha_{R}$ & Indep. & Indep. & yes & Opt. Pert. Theo. & {$[382]$} \\
\hline 1.77 & freezes & gh.-gl. vertex & $\overline{M S}, \mathrm{MOM}$ & Landau & yes & Gribov-Zwanziger & {$[345,208]$} \\
\hline $7.7 \pm 2$ & freezes & - & - & Landau & no & FRG & {$[337]$} \\
\hline 2.97 & freezes & gh.-gl. vertex & - & Landau & no & FRG & {$[250]$} \\
\hline$\sim 4$ & freezes & - & Indep. & Indep. & yes & Analytic Ap. $\Lambda=0.56$ & {$[160]$} \\
\hline 1.25 & mono. decr. & - & $(\overline{M S})$ & Indep. & yes & Analytic Ap. $\Lambda=0.32$ & {$[356,353]$} \\
\hline diverges & $1 / Q^{2}$ & - & Indep. & Indep. & yes & Analytic Approach & {$[362,371]$} \\
\hline 0.47 & freezes & - & Indep. & Indep. & yes & Analytic Approach & {$[372]$} \\
\hline $0.37-0.60$ & freezes & pQCD form & $\overline{M S}$ & Indep. & no & $\mathrm{BPT}$ & {$[212,380]$} \\
\hline 0.81 & freezes & $\mathrm{pQCD}$ form & $\mathrm{V}$ & Indep. & yes & $\mathrm{BPT}$ & {$[44]$} \\
\hline $0.97 \pm 0.44$ & freezes & $\mathrm{pQCD}$ form & $\mathrm{V}$ & Indep. & yes & $\mathrm{BPT}$ & {$[44,381]$} \\
\hline $0.50 \pm 0.08$ & freezes & pQCD form & $\overline{M S}$ & indep. & yes & duality. & {$[383]$} \\
\hline 0 & vanishes & gh.-gl. vertex & - & - & no & $\phi^{4}-Y M$ mapping & {$[384]$} \\
\hline 0 & vanishes & pQCD form & $\overline{M S}$ & indep. & no & Bogoliubov comp. & {$[164]$} \\
\hline 0 & vanishes & $\mathrm{pQCD}$ form & $\overline{M S}$ & Landau & yes & Curci-Fermi Model & {$[386]$} \\
\hline
\end{tabular}

Table 5.3: Continuation of Table 5.2. The references for the seventh row are $[319,320,326$, $318,351,317]$. 
in the instant form [124]. Thus the apparent divergence of the running coupling derived using the potential ( $V$-scheme) may stem from the impossibility to consistently define an effective charge from static heavy-quark interactions because the perturbative QCD contributions of "H diagrams" are IR divergent, as first noted by Appelquist, Dine and Muzinich [7]. This suggests that the $\alpha_{s}\left(Q^{2}\right) \overrightarrow{Q^{2} \rightarrow 0} \infty$ group of schemes could be united with the $\alpha_{s}(0) \sim 0.7$ and $\alpha_{s}(0) \sim 3$ groups if compared within a consistent framework.

The $\alpha_{s}(0)=0$ group It is unclear how to reconcile the three previous groups of solutions for the running coupling with a decoupling model in which the coupling vanishes in the IR: $\alpha_{s}(0)=0$. Only one solution underlies hadron physics, and thus either $\alpha_{s}(0)=0$ or the three other reconcilable groups are irrelevant. We note that the IRfreezing solution is supported by measurements of effective charges such as $\alpha_{g_{1}}, \alpha_{F 3}$ and $\alpha_{\tau}$, and it is a necessary ingredient of the AdS/QCD approach which provides a successful description of hadron properties. Furthermore, the decoupling solution is not compatible with the Kugo-Ojima confinement criterion. Other arguments disfavoring the decoupling solution (violation of the BRST symmetry and unconfined gluons) are given in [261]. On the other hand, an argument for the decoupling solution is that it appears to have a lower action than the freezing solution [266]. It is possible that both solutions are realized in Nature, that the decoupling solution does not lead to confinement or lead to a hadron of larger size than that with a coupling following the scaling solution. In that case, the consequence of the the decoupling solution would not be observed in spite of its possibly lower action.

\section{What is the best definition for $\alpha_{s}$ ?}

An important principle of the renormalization group is that predictions for observables cannot depend on theoretical conventions such as the RS, the initial scale, or the choice of effective charge. Predictions for observables must thus also be independent of the choice of the definition for $\alpha_{s}$. This implies that different choices for the running coupling must be related to each other; for example "Commensurate scale relations" interconnect different couplings in the high $Q^{2}$ pQCD regime.

The optimal choice for the definition of $\alpha_{s}\left(Q^{2}\right)$ at all scales is an unsettled question: there are many ways to define a coupling which satisfies the RGE. This is also the case in QED; nevertheless, the Gell Mann-Low definition -the effective charge defined from elastic scattering of heavy charges- is universally used. However, it would in principle be

possible to choose an alternative formal choice of coupling in QED based on dimensional 
regularization such as $\alpha_{\overline{M S}}$.

To help in assessing the optimal choice of IR-definition for $\alpha_{s}\left(Q^{2}\right)$, we summarize in Table 5.4 various definitions for $\alpha_{s}$, together with their properties and relative advantages. In addition to this table, we can list other desirable, but not necessarily fundamental, properties:

- Interpretability: the coupling should be easily interpretable, as is the case for QED.

- Simplicity: it should be relatively easy to produce the coupling.

- Finiteness: the coupling should be finite at all scales. Since predictions for observables must be finite, this condition implies that if $\alpha_{s}\left(Q^{2}\right)$ diverges, it must be compensated by other divergent factors or terms not included in its primary definition. Although the cancelation of infinities is theoretically possible, the identification of the individual diverging effects cannot be done solely from physical criteria. In addition, from the practical point of view, the cancelation of large quantities greatly increases systematic uncertainties.

This discussion does not imply that only one definition or approach should be used to study $\alpha_{s}$ in the IR domain. A variety of approaches is useful since different techniques often give different perspectives. However, it would be desirable to present results in a commonly agreed definition of the QCD coupling (and scheme and $N_{C}$ and $n_{f}$ values) in order to assure the clarity and consistency of this field of study. Table 5.4 may help to choose a convenient definition for such reference. 


\begin{tabular}{|c|c|c|c|c|c|c|}
\hline Definition & Analytic & $\begin{array}{c}\text { RS- } \\
\text { indep. }\end{array}$ & $\begin{array}{c}\text { Gauge- } \\
\text { indep. }\end{array}$ & Universal & $\begin{array}{c}\text { Based on } \\
\text { first principles }\end{array}$ & $\begin{array}{c}\text { IR- } \\
\text { finite }\end{array}$ \\
\hline \hline pQCD, $\alpha_{p Q C D}$ & yes & no & depends ${ }^{1}$ & yes & yes & no (Landau pole) \\
\hline Eff. charge, $\alpha_{g_{1}}, \alpha_{\tau}, \alpha_{A d S}$ & yes & yes & yes & yes $^{2}$ & yes & yes (freezes) \\
\hline Static quark potential & yes & yes & yes & yes & no & no \\
\hline $\begin{array}{c}\text { Vertices, } \alpha_{s}^{g h}, \\
\alpha_{s}^{q-g}, \alpha_{s}^{3 g}, \alpha_{s}^{4 g}\end{array}$ & yes & no & no & $\begin{array}{c}\text { in } \\
\text { principle }\end{array}$ & yes & $\begin{array}{c}\text { yes (freezes or } \\
\text { vanishes) }\end{array}$ \\
\hline Quark model/Spectroscopy & yes & no & yes & yes & no & yes (freezes) \\
\hline Analytical coupling & yes & no & yes & yes & no & yes (freezes) \\
\hline Dispersive coupling & yes & no & yes & yes & yes & yes (freezes) \\
\hline OPT & yes & yes & yes & unclear & no & yes (freezes) \\
\hline Duality & no & no & yes & unclear & no & yes (freezes) \\
\hline$\lambda \phi^{4} \rightarrow$ Yang Mills mapping & yes & no & unclear & yes & no & yes (vanishes) \\
\hline Curci-Fermi Model & yes & no & no & no & yes & yes (vanishes) \\
\hline
\end{tabular}

Table 5.4: Summary of various definitions of $\alpha_{s}$. "Universal" refers to a unique coupling able to describe any observable or vertex.

Notes:

${ }^{1}$ Depends on the RS. E.g. the coupling in $\overline{M S}$ is gauge-independent but not necessarily the one in a MOM gauge.

${ }^{2}$ Effective charges can be related in the perturbative domain using the CSR, see Section 3.7.1. Those relations are continued in the IR using the method described in Section 5.2.

${ }^{3}$ In principle, $\alpha_{s}^{g h}, \alpha_{s}^{q-g}, \alpha_{s}^{3 g}$, and $\alpha_{s}^{4 g}$ can be related. Also, two solutions (scaling and decoupling, see Section 4.4.3) exist, without consensus on which one is realized in Nature.

${ }^{4}$ Demanding causality as a criterion to define the coupling is not a first principle since the coupling is not necessarily an observable. 


\section{Chapter 6}

\section{Conclusions}

The QCD coupling $\alpha_{s}\left(Q^{2}\right)$ plays a fundamental role in hadron, nuclear, and particle physics, setting the strength of quark and gluon interactions over the entire range of momentum transfer $Q$. The analytic dependence of the coupling in $Q^{2}$ is determined by its logarithmic derivative, the QCD $\beta$-function, which not only incorporates the physics of asymptotic freedom at large momentum transfer, but also the nonperturbative dynamics underlying color confinement. It is possible that the QCD and electroweak running couplings could merge at very high momentum transfers, reflecting the unifying physics of a grand unified theory [30].

In this article, we have reviewed the present theoretical and phenomenological understanding of $\alpha_{s}$, both at high- and low-momentum transfer. We attempted to be both pedagogical and comprehensive, although we had to leave out interesting topics such as the coupling in the $Q^{2}<0$ time-like domain, in space-times of dimension other than 4 , or studies of the coupling at non-zero temperature.

Remarkable progress has been made in the last decade determining the QCD coupling's strength at high momentum transfer using phenomenological input from collider experiments, together with extensive high-order theoretical perturbative computations. Perturbative QCD calculations typically use dimensional regularization to control ultraviolet divergences of loop integrals. It has thus become conventional to adopt the $\overline{M S}$ definition of the coupling. The value of $\alpha_{\overline{M S}}\left(Q^{2}=M_{Z}^{2}\right)$ has been determined from various experiments to remarkable precision and using different processes, providing an important test of the validity of QCD. High precision theoretical determinations of $\alpha_{s}$ from lattice gauge theory have also been obtained. Although some tension still exists between various determinations, it probably reflects too optimistic estimates of systematic uncertainties, rather than new physics or a problem associated with QCD itself. 
As we have emphasized, the choice of the $\overline{M S}$ scheme is only a convention. Other renormalization schemes are possible, such as the MOM scheme, although its use is problematic because of its dependence on the choice of gauge.

It has also become conventional to guess the renormalization scale $\mu_{R}$ for the $\overline{M S}$ coupling scheme. The most common choice is to set $Q^{2}=\mu^{2}$ because it yields the simplest perturbative expansions of observables. The setbacks are convergence issues with the expansion series and an unintuitive interpretation of the coupling. Although the choice of the simplicity criterium is arbitrary, one expects that the dependence of the pQCD predictions on this guess become diminished at high order. However, this expectation conflicts with the $\alpha_{s}^{n} \beta_{0}^{n} n$ ! divergent renormalon growth of the pQCD series. The fixed order pQCD predictions are scheme-dependent if one uses an arbitrary scale for $\mu_{R}$. This is at odds with an important principle - "renormalization group invariance": theoretical predictions cannot depend on theoretical conventions, such as the choice of scheme or the choice of the renormalization scale.

As we have discussed, none of these problems appear when one uses the "Principle of Maximum Conformality" $(P M C)$ to set the renormalization scale order-by-order in perturbation theory. As in QED, all terms in the $\mathrm{pQCD}$ series involving the $\beta$-function can be summed into the running coupling by shifting the renormalization scale at each order; this sets the argument of the coupling at each order, and the resulting coefficients of the series then match the coefficients of the corresponding conformal theory with $\beta=0$. The $\beta_{i}$ terms can be identified unambiguously at each order by introducing an extra parameter $\delta$ in the subtraction that defines the $\overline{M S}$ scheme.

The use of the $P M C$ thus eliminates renormalization-scale ambiguities, eliminates renormalon divergences, and -most important- gives predictions which are independent of the choice of renormalization scheme. Also, as in QED, the PMC renormalization scales reflect the virtuality of the gluon propagators and correctly set the number $n_{f}$ of contributing quark flavors at each order. For example, the reduced virtuality of the two $s$-channel gluons in the $q \bar{q} \rightarrow g g \rightarrow t \bar{t}$ amplitude can account for the large $t \bar{t}$ asymmetry in the $\bar{p} p \rightarrow t \bar{t} X$ observed at colliders [60,407]. Nonzero quark masses can be retained so that the $\beta$-function remains analytic as it passes through each flavor threshold [30].

Applying the PMC also provides "Commensurate Scale Relations," between QCD observables which have no renormalization scale or scheme ambiguities. A classic example is the "Generalized Crewther Relation" which connects the QCD corrections to the Bjorken sum rule, which is measured in polarized deep inelastic lepton-nucleon scattering, to the QCD corrections to the annihilation cross section ratio $R_{e^{+} e^{-}}(s)$. Again 
there are no renormalization scale ambiguities.

In the second part of the review, we have discussed a much more complex problem, the behavior of the QCD coupling $\alpha_{s}\left(Q^{2}\right)$ at low momentum transfers, where even its definition is not universally agreed upon. We have described a number of nonperturbative approaches for defining and computing the coupling at small $Q^{2}$, such as approximately solving the Dyson-Schwinger equations and the use of different assumptions for its analytic form. The various analyses reported in the literature yield predictions for $\alpha_{s}\left(Q^{2}=0\right)$ which range from zero to infinity.

The different theoretical and analytical approaches for analyzing the QCD coupling can be classified within three groups: models where $\alpha_{s}\left(Q^{2}=0\right)$ vanishes, models where $\alpha_{s}\left(Q^{2}\right)$ diverges as $Q^{2} \rightarrow 0$, and models where $\alpha_{s}\left(Q^{2}\right)$ remains roughly constant and of moderate value in the IR. These models reflect the range of possible definitions of $\alpha_{s}$ itself, the choice of renormalization scheme, and whether or not one introduces the concept of an effective nonzero gluon mass or equivalent momentum scales.

An important tool for resolving the ambiguity in defining $\alpha_{s}\left(Q^{2}\right)$ is the use of "effective charges": a coupling defined directly from a perturbatively calculable observable. By definition an effective charge is finite at all scales. A natural choice would be to define the QCD coupling from the heavy-quark potential as a generalization of the traditional Gell-Mann-Low coupling used in QED. However, this coupling, called $\alpha_{V}\left(Q^{2}\right)$, requires an infrared cutoff because of " $\mathrm{H}$ " graph contributions arising from non-Abelian corrections to multi-gluon exchange diagrams.

A satisfactory choice for defining the QCD coupling is the effective charge $\alpha_{g_{1}}\left(Q^{2}\right)$ which is obtained from the sum of QCD radiative corrections to the Bjorken sum rule. It is infrared finite and well measured, both at low and high $Q^{2}$. The behavior of $\alpha_{g_{1}}\left(Q^{2}\right)$ at high $Q^{2}$ is known to four loops in pQCD.

Remarkably, all of the measurements of $\alpha_{g_{1}}\left(Q^{2}\right)$ for $Q^{2}<1 \mathrm{GeV}^{2}$ are consistent with the nonperturbative prediction from AdS/QCD and light-front holography:

$$
\frac{\alpha_{g_{1}}\left(Q^{2}\right)}{\pi}=\exp \left(\frac{-Q^{2}}{4 \kappa^{2}}\right)
$$

where $\kappa$ can be determined by the proton mass, $\kappa=M_{p} / 2$, or other hadron masses. The same theory successfully predicts the Regge spectroscopy of the light mesons and baryons, as well as their dynamical structure, such as the light-front wavefunctions underlying the hadron from factors, structure functions, and distribution amplitudes. The value of $\kappa$ also determines the confining potential for light quarks in the frame- 
independent light-front Hamiltonian.

It is also remarkable that by matching at a scale $Q_{0}$ the value of $\alpha_{g_{1}}\left(Q^{2}\right)$ and its derivative obtained from light-front holographic QCD to the predictions of pQCD, one can determine $\Lambda_{\overline{M S}}$ from the value of $\kappa$. The result of this matching is in agreement with the value of $\Lambda_{\overline{M S}}$ determined from high energy physics phenomenology. The value of the matching scale: $Q_{0}^{2} \simeq 1.25 \mathrm{GeV}^{2}$ in the $g_{1}$ scheme can be interpreted as the transition scale between the perturbative and nonperturbative domains of QCD. It is thus natural to adopt $Q_{0}^{2}$ as the starting scale for the DGLAP evolution of structure functions and the ERBL evolution of hadronic distribution amplitudes. The light front holographic QCD analysis can also be used to complement and constrain other nonperturbative approaches to QCD.

Thus QCD and the study of its running coupling have now entered a new domain, where predictions for hadronic phenomena can be made over all scales. We hope that this review has illuminated the physics underlying the remarkable progress in this field.

Acknowledgments We thank Hans Guenter Dosch, Cedric Lorcé, Matin Mojaza, Christian Weiss, and Xing-Gang Wu for instructive discussions on $\alpha_{s}$ and related topics. We are grateful to A. Faessler for his invitation to write this review. This material is based upon work supported by the U.S. Department of Energy, Office of Science, Office of Nuclear Physics under contract DE-AC05-06OR23177 and DE-AC02-76SF00515. SLAC-PUB-16448. 


\section{Appendix A}

\section{Lexicon}

To make this review accessible to non-specialists, we list here some of the specific vocabulary associated with studies of the QCD coupling, with brief explanations and references to where it is first discussed in the review.

- Asymptotic series; Poincaré series. See also "renormalons". A series that converges up to an order $k$ and then diverges. The series reaches its best convergence at order $N_{b}$ and then diverges for orders $N \gtrsim N_{b}+\sqrt{N_{b}}$. Quantum Field Theory series typically are asymptotic and converge up to an order $N_{b} \simeq 1 / a$, with $a$ the expansion coefficient. IR renormalons generate an $n ! \beta^{n}$ factorial growth of the $n^{\text {th }}$ coefficients in nonconformal $(\beta \neq 0)$ theories. Perturbative calculation to high order $\left(\alpha_{s}^{20}\right)$ has been performed on the Lattice [408] to check the asymptotic behavior of QCD series. Factorial growth is seen up to the $20^{\text {th }}$ order of the calculated series.

- $\beta$-function. The logarithmic derivative of $\alpha_{s}: \beta\left(\mu^{2}\right)=\frac{d \alpha_{s}(\mu)}{d \ln (\mu)}$ where $\mu$ is the subtraction point. In the perturbative domain, $\beta$ can be expressed as a perturbative series $\beta=-\frac{1}{4 \pi} \sum_{n=0}\left(\frac{\alpha_{s}}{4 \pi}\right)^{n} \beta_{n}$. (See Section 3.2).

- Commensurate Scale Relations (CSR). Relations linking two effective charges obtained from different observables or two effective couplings expressed in different renormalization schemes. Since BLM/PMC scale setting is used, all terms involving the coefficients $\beta_{n}$ do not appear in the CSR series coefficients, eliminating the renormalon divergence (see "Asymptotic series" and "renormalons"). The convergence property is thus superior compared to the more straightforward relations obtained by equating the perturbative expressions of the two couplings. (See Section 3.7.1). 
- Condensate (or Vacuum Expectation Value, VEV). The vacuum expectation value of a given local operator. Condensates allow one to parameterize the nonperturbative OPE's power corrections. Condensates and vacuum loop diagrams do not appear in the frame-independent light-front Hamiltonian since all lines have $k^{+}=k^{0}+k^{3} \geq 0$ and the sum of + momenta is conserved at every vertex. In the light-front formalism condensates are associated with physics of the hadron wave function and are called "in-hadron" condensates, which refers to physics possibly contained in the higher LF Fock states of the hadrons [409]. In the case of the Higgs theory, the usual Higgs VEV of the instant form Hamiltonian is replaced by a "zero mode", a background field with $k^{+}=0$ [410].

- Conformal behavior/theory. The behavior of a quantity or a theory that is scale invariant. In a conformal theory the $\beta$-function vanishes. More rigorously, a conformal theory is invariant under both dilatation and the special conformal transformations which involves inversion. (See Sections 3.9.6 and 4.2).

- Couplant: the normalized coupling, defined as $\alpha_{s} / \pi$.

- Decoupling solution. See also scaling solution. One of the two classes of solutions for couplings defined using vertices. The decoupling solution leads to an IR-vanishing $\alpha_{s}$. (See Section 4.4.3).

- Dimensional transmutation: The emergence of a mass or momentum scale in the quantum theory with a classical Lagrangian devoid of explicit mass or energy parameters [13]. (See Section 3.1).

- Effective coupling. The renormalized (running) coupling, in contrast with the constant bare coupling. (See Section 2).

- Effective charge. An effective coupling defined from a perturbatively calculable observable. It includes all perturbative and relevant nonperturbative effects. (See Sections 3.7.1 and 4.1).

- Freezing. The loss of scale dependence of finite $\alpha_{s}$ in the infrared. See also conformal behavior.

- Green's function, n-point Green's function. The function which describes the propagation of a field between $n$ space-time points. 
- Higher-twist (see also "Twist"), $1 / Q^{n}$ power corrections, typically derived from the $O P E$ analysis of the nonperturbative effects of multiparton interactions. Highertwist is sometimes interpretable as kinematical phenomena, e.g. the mass $M$ of a nucleon introduces a power correction beyond the pQCD scaling violations, or as dynamical phenomena, e.g. the intermediate distance transverse forces that confine quarks [167]. (See Section 4.1).

- Infrared fixed point. The value of the momentum transfer scale $\mu$ where $\beta(\mu)=0$. This implies either that $\alpha_{s}$ either freezes in the IR $(\beta(\mu)$ remains 0 beyond the IR fixed point), or that it vanishes $(\beta(\mu)$ becomes positive passed the IR fixed point).

- Infrared exponent. The power-law exponent which describes how the ghost and gluon propagators scale with $Q^{2}$. (See Section 4.4.3).

- Kugo-Ojima confinement criterion [269]. A condition that ensures the conservation of the global color charge. It is a necessary but not a sufficient criterion for confinement. It implies that the Fadeev-Popov ghost propagator behavior in the low energy regime is more singular than $1 / Q^{2}$, and that the gluon propagator is less singular; see e.g., Refs. [204, 222]. In such a case, the physical space of states can contain only color singlets, i.e., the theory is confining.

- Landau pole; Landau singularity; Landau ghost. The point where a perturbative coupling diverges. At first order (1-loop) in pQCD, this occurs at the scale parameter $\Lambda$. The value can depend on the choice of renormalization scheme, the order $\beta_{i}$ at which the coupling series is estimated, the number of flavors $n_{f}$ and the approximation chosen to solve Eq. (3.6) for orders higher than $\beta_{1}$. The Landau pole is unphysical. (See Section 3.6).

- $(n-)$ Loops. The order of perturbation theory appearing in the coupling calculation; i.e., the order of the $\beta$-series. In the case of $\mathrm{QED}$, it is the order of vacuum polarization loops contributing to the renormalization of the coupling. The $n$-loop approximation corresponds to a $\beta$-series truncated to order $\beta_{n-1}$. (See Section $3.4)$.

- Operator Product Expansion (OPE). See also higher- twist. The OPE uses the twist of effective operators to predict the power-law fall-off of an amplitude. It thus can be used to distinguish logarithmic leading twist perturbative corrections 
from the $1 / Q^{n}$ power corrections. The OPE typically does not provide values for the nonperturbative power correction coefficients. (See Section 4.1).

- Pinch technique. A method which adds external on-shell legs to ensure the gauge invariance of an amplitude. For example, the calculation of the three-gluon vertex becomes gauge invariant if the coupling to three on-shell external quark lines are included. See Refs. [30, 278]

- Principle of Maximal Conformality (PMC). A method used to set the renormalization scale, order-by-order in perturbation theory, by shifting all $\beta$ terms in the pQCD series into the renormalization scale of the running QCD coupling at each order. The resulting coefficients of the series then match the coefficients of the corresponding conformal theory with $\beta=0$. The PMC generalizes the Brodsky Lepage Mackenzie BLM method to all orders. In the Abelian $N_{C} \rightarrow 0$ limit, the PMC reduces to the standard Gell-Mann-Low method used for scale setting in QED [147].

- Power corrections. See "Higher- twist" and "Renormalons".

- Pure gauge sector; pure Yang Mill; pure field. Non Abelian field theory without fermions. See also quenched approximation.

- Quenched approximation. Calculations where the fermion loops are neglected. It differs from the pure gauge, pure Yang Mills case in that heavy (static) quarks are present.

- Renormalization scale. The argument of the running coupling. See also "Subtraction point".

- Renormalon. The residual between the physical value of an observable and the Asymptotic series of the observable at its best convergence order $n \simeq 1 / \alpha_{s}$. The terms of a pQCD calculation which involve the $\beta$-function typically diverge as $n !$; i.e., as a renormalon. Borel summation techniques indicate that IR renormalons can often be interpreted as power corrections. Thus, IR renormalons should be related to the higher twist corrections of the OPE formalism [411]. The existence of IR renormalons in pure gauge QCD is supported by Lattice QCD [408]. See also "Asymptotic series". 
- Scale-fixing. The renormalization scale at each order of perturbation theory is set by the BLM/PMC method by absorbing the contributing $\beta$ terms. In the case of QED, scale-fixing resums the vacuum polarization contributions to the photon propagator.

- QCD Scale parameter $\Lambda$. The UV scale ruling the energy-dependence of $\alpha_{s}$. It also provides the scale at which $\alpha_{s}$ is expected to be large and nonperturbative treatment of QCD is required. (See Section 3.2).

- Scaling solution. See also decoupling solution. One of the two classes of solutions for couplings defined using vertices. The scaling solution results in an IR-freezing of $\alpha_{s}$. (See Section 4.4.3).

- Slavnov-Taylor identities [32]: The non-Abelian generalization of the Ward-Takahashi identities. (See Section 3.4).

- Subtraction point $\mu$. The scale at which the renormalization procedure subtracts the UV divergences. (See Section 3.2).

- Tadpole corrections. In the context of lattice QCD, tadpole terms are unphysical contributions to the lattice action which arise from the discretization of space-time. They contribute at NLO of the bare coupling $g^{\text {bare }}=\sqrt{4 \pi \alpha_{s}^{\text {bare }}}$ to the expression of the gauge link variable $U_{\vec{\mu}}$. (The LO corresponds to the continuum limit). To suppress these contributions, one can redefine the lattice action by adding larger Wilson loops or by rescaling the link variable.

- Twist. The twist of an elementary operator is given by its dimension minus its spin. For example, the quark operator $\psi$ has dimension 3 , spin $1 / 2$ and thus twist $=1$. It is also the number of constituents of a hadron. See "Higher-twists" .

- Unquenched QCD. See pure gauge sector and quenched approximation.

- Wilson Loops. Closed paths linking various sites in a lattice [103]. They are used to define the lattice action. (See Section 3.9.4). 


\section{Bibliography}

[1] K. A. Olive et al. [Particle Data Group Collaboration], Review of Particle Physics, Chin. Phys. C 38, 090001 (2014).

[2] G. M. Prosperi, M. Raciti and C. Simolo, On the running coupling constant in QCD, Prog. Part. Nucl. Phys. 58, 387 (2007) [hep-ph/0607209].

[3] G. Altarelli, The QCD running coupling and its measurement, PoS Corfu 2012, 002 (2013) [arXiv:1303.6065 [hep-ph]].

[4] G. Grunberg, Renormalization group improved perturbative QCD, Phys. Lett. B 95, 70 (1980) [Phys. Lett. B 110, 501 (1982)]; Renormalization scheme independent QCD and QED: The method of effective charges, Phys. Rev. D 29, 2315 (1984); On some ambiguities in the method of effective charges, Phys. Rev. D 40, 680 (1989).

[5] S. J. Brodsky and G. F. de Teramond, Light-front dynamics and AdS/QCD correspondence: The pion form factor in the space- and time-like regions, Phys. Rev. D 77, 056007 (2008) [arXiv:0707.3859 [hep-ph]].

[6] S. J. Brodsky and R. Shrock, Maximum wavelength of confined quarks and gluons and properties of quantum chromodynamics, Phys. Lett. B 666, 95 (2008) [arXiv:0806.1535 [hep-th]].

[7] T. Appelquist, M. Dine and I. J. Muzinich, The static potential in quantum chromodynamics, Phys. Lett. B 69, 231 (1977); The static limit of quantum chromodynamics, Phys. Rev. D 17, 2074 (1978).

[8] B. A. Kniehl, A. A. Penin, V. A. Smirnov and M. Steinhauser, Potential NRQCD and heavy quarkonium spectrum at next-to-next-to-next-to-leading order, Nucl. Phys. B 635, 357 (2002) [hep-ph/0203166]; A. V. Smirnov, V. A. Smirnov and M. Steinhauser, Three-loop static potential, Phys. Rev. Lett. 104, 112002 (2010) [arXiv:0911.4742 [hep-ph]]. 
[9] J. M. Maldacena, The Large N limit of superconformal field theories and supergravity, Int. J. Theor. Phys. 38, 1113 (1999) [Adv. Theor. Math. Phys. 2, 231 (1998)] [hep-th/9711200].

[10] C. G. Bollini and J. J. Giambiagi, Dimensional renormalization: The number of dimensions as a regularizing parameter, Nuovo Cim. B 12, 20 (1972).

[11] G. 't Hooft, Dimensional regularization and the renormalization group, Nucl. Phys. B 61, 455 (1973).

[12] W. A. Bardeen, A. J. Buras, D. W. Duke and T. Muta, Deep inelastic scattering beyond the leading order in asymptotically free gauge theories, Phys. Rev. D 18, 3998 (1978).

[13] S. R. Coleman and D. J. Gross, Price of asymptotic freedom, Phys. Rev. Lett. 31, 851 (1973).

[14] C. G. Callan, Jr., Broken scale invariance in scalar field theory, Phys. Rev. D 2, 1541 (1970); K. Symanzik, Small distance behavior in field theory and power counting, Commun. Math. Phys. 18, 227 (1970); Small distance behavior analysis and Wilson expansion, Commun. Math. Phys. 23, 49 (1971).

[15] D. J. Gross and F. Wilczek, Ultraviolet behavior of nonabelian gauge theories, Phys. Rev. Lett. 30, 1343 (1973); H. D. Politzer, Reliable perturbative results for strong interactions?, Phys. Rev. Lett. 30, 1346 (1973).

[16] W. E. Caswell, Asymptotic behavior of nonabelian gauge theories to two-loop order, Phys. Rev. Lett. 33, 244 (1974).

[17] D. R. T. Jones, Asymptotic behavior of supersymmetric Yang-Mills theories in the two-loop approximation, Nucl. Phys. B 87, 127 (1975); E. Egorian and O. V. Tarasov, Two-loop renormalization of the QCD in an arbitrary gauge, Teor. Mat. Fiz. 41, 26 (1979) [Theor. Math. Phys. 41, 863 (1979)].

[18] O. V. Tarasov, A. A. Vladimirov and A. Y. Zharkov, The Gell-Mann-Low function of QCD in the three-loop approximation, Phys. Lett. B 93, 429 (1980); S. A. Larin and J. A. M. Vermaseren, The three-loop QCD beta function and anomalous dimensions, Phys. Lett. B 303, 334 (1993) [hep-ph/9302208]. 
[19] T. van Ritbergen, J. A. M. Vermaseren and S. A. Larin, The four-loop beta function in quantum chromodynamics, Phys. Lett. B 400, 379 (1997) [hep-ph/9701390].

[20] K. G. Chetyrkin, P. A. Balkov and J. H. Khn, Towards QCD running in 5 loops: quark mass anomalous dimension, PoS RADCOR 2013, 056 (2013) [arXiv:1402.6606 [hep-ph]].

[21] W. E. Caswell and F. Wilczek, On the gauge dependence of renormalization group parameters, Phys. Lett. B 49, 291 (1974).

[22] W. Celmaster and R. J. Gonsalves, The renormalization prescription dependence of the QCD coupling constant, Phys. Rev. D 20, 1420 (1979).

[23] W. Celmaster and R. J. Gonsalves, QCD perturbation expansions in a coupling constant renormalized by momentum space subtraction, Phys. Rev. Lett. 42, 1435 (1979); R. Barbieri, L. Caneschi, G. Curci and E. d'Emilio, Scaling violations and perturbative quantum chromodynamics, Phys. Lett. B 81, 207 (1979).

[24] P. Boucaud, F. de Soto, J. P. Leroy, A. Le Yaouanc, J. Micheli, H. Moutarde, O. Pene and J. Rodriguez-Quintero, Artefacts and $\left\langle A^{2}\right\rangle$ power corrections: Revisiting the MOM $Z_{\psi}\left(p^{2}\right)$ and $Z_{V}$, Phys. Rev. D 74, 034505 (2006) [hep-lat/0504017]; P. Boucaud, F. De Soto, J. P. Leroy, A. Le Yaouanc, J. Micheli, O. Pene and J. Rodriguez-Quintero, Ghost-gluon running coupling, power corrections and the determination of $\Lambda_{\overline{M S}}$, Phys. Rev. D 79, 014508 (2009) [arXiv:0811.2059 [hep-ph]].

[25] K. G. Chetyrkin and A. Retey, Three loop three linear vertices and four loop similar to MOM beta functions in massless QCD, hep-ph/0007088; L. von Smekal, K. Maltman and A. Sternbeck, The Strong coupling and its running to four loops in a minimal MOM scheme, Phys. Lett. B 681, 336 (2009) [arXiv:0903.1696 [hep$\mathrm{ph}]]$.

[26] E. Gardi, G. Grunberg and M. Karliner, Can the QCD running coupling have a causal analyticity structure?, JHEP 9807, 007 (1998) [hep-ph/9806462].

[27] D. A. Barry, J.-Y. Parlange, L. Li, H. Prommer, C. J. Cunningham, F. Stagnitti, Analytical approximations for real values of the Lambert $W$ function, Math. Comp. Simul. 5395 (2000). 
[28] K. G. Chetyrkin, B. A. Kniehl and M. Steinhauser, Strong coupling constant with flavor thresholds at four loops in the MS scheme, Phys. Rev. Lett. 79, 2184 (1997) [hep-ph/9706430].

[29] S. A. Larin, T. van Ritbergen and J. A. M. Vermaseren, The large quark mass expansion of $\Gamma\left(Z_{0} \rightarrow\right.$ hadrons $)$ and $\Gamma\left(\tau \rightarrow \nu_{\tau}+\right.$ hadrons $)$ in the order $\alpha_{s}^{3}$, Nucl. Phys. B 438, 278 (1995) [hep-ph/9411260].

[30] M. Binger and S. J. Brodsky, Physical renormalization schemes and grand unification, Phys. Rev. D 69 (2004) 095007.

[31] L. D. Faddeev and V. N. Popov, Feynman diagrams for the Yang-Mills field, Phys. Lett. B 25, 29 (1967).

[32] J. C. Taylor, Ward identities and charge renormalization of the Yang-Mills field, Nucl. Phys. B 33, 436 (1971); A. A. Slavnov, Ward identities in gauge theories, Theor. Math. Phys. 10, 99 (1972) [Teor. Mat. Fiz. 10, 153 (1972)].

[33] See. e.g. M. Gockeler, R. Horsley, V. Linke, P. E. L. Rakow, G. Schierholz and H. Stuben, Is there a Landau pole problem in QED?, Phys. Rev. Lett. 80, 4119 (1998) [hep-th/9712244].

[34] S. J. Brodsky, E. Gardi, G. Grunberg and J. Rathsman, Disentangling running coupling and conformal effects in QCD, Phys. Rev. D 63, 094017 (2001) [hep$\mathrm{ph} / 0002065]$.

[35] M. Gell-Mann and F. E. Low, Quantum electrodynamics at small distances, Phys. Rev. 95, 1300 (1954).

[36] J. D. Bjorken, Applications of the Chiral $U(6) \times U(6)$ Algebra of Current Densities, Phys. Rev. 148, 1467 (1966); Inelastic Scattering of Polarized Leptons from Polarized Nucleons, Phys. Rev. D 1, 1376 (1970).

[37] A. L. Kataev, The Ellis-Jaffe sum rule: The estimates of the next to next-toleading order QCD corrections, Phys. Rev. D 50, 5469 (1994) [hep-ph/9408248]; P. A. Baikov, K. G. Chetyrkin and J. H. Kuhn, Adler function, Bjorken sum rule, and the Crewther relation to order $\alpha_{s}^{4}$ in a general gauge theory, Phys. Rev. Lett. 104, 132004 (2010) [arXiv:1001.3606 [hep-ph]]. 
[38] S. L. Adler, Some simple vacuum polarization phenomenology: $e^{+} e^{-} \rightarrow$ hadrons: The muonic-atom x-ray discrepancy and $g_{\mu}-2$, Phys. Rev. D 10, 3714 (1974).

[39] S. J. Brodsky, S. Menke, C. Merino and J. Rathsman, On the behavior of the effective QCD coupling $\alpha_{\tau}(s)$ at low scales, Phys. Rev. D 67, 055008 (2003) [hep$\mathrm{ph} / 0212078]$.

[40] S. J. Brodsky, Commensurate scale relations and the Abelian correspondence principle, hep-ph/9806445.

[41] W. W. Repko, M. D. Santia and S. F. Radford, Three-loop static QCD potential in heavy quarkonia, Nucl. Phys. A 924, 65 (2014) [arXiv:1211.6373 [hep-ph]].

[42] W. Fischler, Quark-anti-quark potential in QCD, Nucl. Phys. B 129, 157 (1977); A. Billoire, How heavy must be quarks in order to build Coulombic $q-\bar{q}$ bound states, Phys. Lett. B 92, 343 (1980); A. Billoire, Another connection between the $\Lambda$ parameters of the Euclidean lattice and continuum QCD, Phys. Lett. B 104, 472 (1981).

[43] M. Peter, The static quark-anti-quark potential in QCD to three loops, Phys. Rev. Lett. 78, 602 (1997) [hep-ph/9610209]; M. Peter, The static potential in QCD: A Full two-loop calculation, Nucl. Phys. B 501, 471 (1997) [hep-ph/9702245]; Y. Schroder, The Static potential in QCD to two loops, Phys. Lett. B 447, 321 (1999) [hep-ph/9812205]; M. Melles, The static QCD potential in coordinate space with quark masses through two loops, Phys. Rev. D 62, 074019 (2000) [hepph/0001295]; M. Melles, Two loop mass effects in the static position space QCD potential, Nucl. Phys. Proc. Suppl. 96, 472 (2001) [hep-ph/0009085]; A. H. Hoang, Bottom quark mass from upsilon mesons: Charm mass effects, hep-ph/0008102; S. Recksiegel and Y. Sumino, Perturbative QCD potential, renormalon cancellation and phenomenological potentials, Phys. Rev. D 65, 054018 (2002).

[44] A. M. Badalian, Strong coupling constant in coordinate space, Phys. Atom. Nucl. 63, 2173 (2000) [Yad. Fiz. 63, 2269 (2000)].

[45] N. Brambilla, A. Pineda, J. Soto and A. Vairo, The Infrared behavior of the static potential in perturbative QCD, Phys. Rev. D 60, 091502 (1999) [hep-ph/9903355]; N. Brambilla, A. Pineda, J. Soto and A. Vairo, Potential NRQCD: An effective theory for heavy quarkonium, Nucl. Phys. B 566, 275 (2000) [hep-ph/9907240]; 
B. A. Kniehl and A. A. Penin, Ultrasoft effects in heavy quarkonium physics, Nucl. Phys. B 563, 200 (1999) [hep-ph/9907489]; A. V. Smirnov, V. A. Smirnov and M. Steinhauser, Fermionic contributions to the three-loop static potential, Phys. Lett. B 668, 293 (2008) [arXiv:0809.1927 [hep-ph]]; A. V. Smirnov, V. A. Smirnov and M. Steinhauser, The Static quark potential to three loops in perturbation theory, Nucl. Phys. Proc. Suppl. 205-206, 320 (2010) [arXiv:1006.5513 [hep-ph]]; C. Anzai, Y. Kiyo and Y. Sumino, Static QCD potential at three-loop order, Phys. Rev. Lett. 104, 112003 (2010) [arXiv:0911.4335 [hep-ph]].

[46] F. A. Chishtie and V. Elias, RG / Pade estimate of the three loop contribution to the QCD static potential function, Phys. Lett. B 521, 434 (2001) [hep-ph/0107052]; A. Pineda, Determination of the bottom quark mass from the $\Upsilon(1 S)$ system, JHEP 0106, 022 (2001) [hep-ph/0105008]; G. Cvetic, Estimate of the three-loop contribution to the QCD static potential from renormalon cancellation, J. Phys. G 30, 863 (2004) [hep-ph/0309262].

[47] I. I. Balitsky, Wilson loop for the stretched contours in vacuum fields and the small distance behavior of the interquark potential, Nucl. Phys. B 254, 166 (1985); U. Aglietti and Z. Ligeti, Renormalons and confinement, Phys. Lett. B 364, 75 (1995) [hep-ph/9503209]; R. Akhoury and V. I. Zakharov, On nonperturbative corrections to the potential for heavy quarks, Phys. Lett. B 438, 165 (1998) [hep$\mathrm{ph} / 9710487]$.

[48] A. Pineda, Review of heavy quarkonium at weak coupling, Prog. Part. Nucl. Phys. 67, 735 (2012) [arXiv:1111.0165 [hep-ph]].

[49] X. Garcia i Tormo, Review on the determination of $\alpha_{s}$ from the QCD static energy, Mod. Phys. Lett. A 28, 1330028 (2013) [arXiv:1307.2238].

[50] S. J. Brodsky and H. J. Lu, Commensurate scale relations in quantum chromodynamics, Phys. Rev. D 51, 3652 (1995) [hep-ph/9405218]; S. J. Brodsky, G. T. Gabadadze, A. L. Kataev and H. J. Lu, The generalized crewther relation in QCD and its experimental consequences, Phys. Lett. B 372, 133 (1996) [hep$\mathrm{ph} / 9512367]$.

[51] X. G. Wu, S. J. Brodsky and M. Mojaza, The renormalization scale-setting problem in QCD, Prog. Part. Nucl. Phys. 72, 44 (2013) [arXiv:1302.0599 [hep-ph]]; S. J. Brodsky, M. Mojaza and X. G. Wu, Systematic scale-setting to all orders: 
The principle of maximum conformality and commensurate scale relations, Phys. Rev. D 89, 014027 (2014) [arXiv:1304.4631 [hep-ph]].

[52] S. J. Brodsky, G. P. Lepage and P. B. Mackenzie, On the elimination of scale ambiguities in perturbative quantum chromodynamics, Phys. Rev. D 28, 228 (1983); G. P. Lepage and P. B. Mackenzie, On the viability of lattice perturbation theory, Phys. Rev. D 48, 2250 (1993) [hep-lat/9209022]; M. Beneke and V. M. Braun, Naive nonAbelianization and resummation of fermion bubble chains, Phys. Lett. B 348, 513 (1995) [hep-ph/9411229]; P. Ball, M. Beneke and V. M. Braun, Resummation of $\left(\beta_{0} \alpha_{s}\right)^{n}$ corrections in QCD: Techniques and applications to the $\tau$ hadronic width and the heavy quark pole mass, Nucl. Phys. B 452, 563 (1995) [hep-ph/9502300].

[53] M. Binger and S. J. Brodsky, The Form-factors of the gauge-invariant three-gluon vertex, Phys. Rev. D 74, 054016 (2006) [hep-ph/0602199].

[54] M. Neubert, Scale setting in QCD and the momentum flow in Feynman diagrams, Phys. Rev. D 51, 5924 (1995) [hep-ph/9412265].

[55] S. V. Mikhailov, Generalization of BLM procedure and its scales in any order of pQCD: A Practical approach, JHEP 0706, 009 (2007) [hep-ph/0411397].

[56] H. H. Ma, X. G. Wu, Y. Ma, S. J. Brodsky and M. Mojaza, Setting the renormalization scale in perturbative QCD: Comparisons of the principle of maximum conformality with the sequential extended Brodsky-Lepage-Mackenzie approach, Phys. Rev. D 91, 094028 (2015) [arXiv:1504.01260 [hep-ph]].

[57] N. Uraltsev, BLM resummation and OPE in heavy flavor transitions, Nucl. Phys. B 491, 303 (1997) [hep-ph/9610425]; A. Czarnecki, K. Melnikov and N. Uraltsev, NonAbelian dipole radiation and the heavy quark expansion, Phys. Rev. Lett. 80, 3189 (1998) [hep-ph/9708372].

[58] S. Catani, B. R. Webber and G. Marchesini, QCD coherent branching and semiinclusive processes at large $x$, Nucl. Phys. B 349, 635 (1991); S. Catani and L. Trentadue, Resummation of the QCD perturbative series for hard processes, Nucl. Phys. B 327, 323 (1989);

[59] S. J. Brodsky and X. G. Wu, Scale setting using the extended renormalization group and the Principle of Maximum Conformality: the QCD coupling constant at four loops, Phys. Rev. D 85, 034038 (2012) [Phys. Rev. D 86, 079903 (2012)] 
[arXiv:1111.6175 [hep-ph]]; Application of the Principle of Maximum Conformality to top-pair production, Phys. Rev. D 86, 014021 (2012) [Phys. Rev. D 87, 099902 (2013)] [arXiv:1204.1405 [hep-ph]]; Eliminating the renormalization scale ambiguity for top-pair production using the Principle of Maximum Conformality, Phys. Rev. Lett. 109, 042002 (2012) [arXiv:1203.5312 [hep-ph]]; S. J. Brodsky and L. Di Giustino, Setting the renormalization scale in QCD: The Principle of Maximum Conformality, Phys. Rev. D 86, 085026 (2012) [arXiv:1107.0338 [hep-ph]]; M. Mojaza, S. J. Brodsky and X. G. Wu, Systematic all-orders method to eliminate renormalization-scale and scheme ambiguities in perturbative QCD, Phys. Rev. Lett. 110, 192001 (2013) [arXiv:1212.0049 [hep-ph]].

[60] S. J. Brodsky and X. G. Wu, Application of the Principle of Maximum Conformality to the top-quark forward-backward asymmetry at the Tevatron, Phys. Rev. D 85, 114040 (2012) [arXiv:1205.1232 [hep-ph]].

[61] P. M. Stevenson, Optimized perturbation theory, Phys. Rev. D 23, 2916 (1981); Optimization and the ultimate convergence of QCD perturbation theory, Nucl. Phys. B 231, 65 (1984); See also J. Kubo, S. Sakakibara and P. M. Stevenson, The renormalization scheme ambiguity and perturbation theory near a fixed point, Phys. Rev. D 29, 1682 (1984); J. Chyla, A. Kataev and S. Larin, Renormalization scheme dependence and infrared behavior in $e^{+} e^{-}$annihilations and $\tau$ lepton decay at the next-to-next-to-leading order of perturbative QCD, Phys. Lett. B 267, 269 (1991); S. J. Brodsky, A. H. Hoang, J. H. Kuhn and T. Teubner, "Angular distributions of massive quarks and leptons close to threshold," Phys. Lett. B 359, 355 (1995) [hepph/9508274]; N. J. Watson, The gauge independent QCD effective charge, Nucl. Phys. B 494, 388 (1997) [hep-ph/9606381]; The Pinch technique beyond one loop: The gauge independent two loop quark self-energy, Nucl. Phys. B 552, 461 (1999) [hep-ph/9812202]; J. Papavassiliou, The Pinch technique at two loops: The case of massless Yang-Mills theories, Phys. Rev. D 62, 045006 (2000) [hep-ph/9912338]; D. Binosi and J. Papavassiliou, The QCD effective charge to all orders, Nucl. Phys. Proc. Suppl. 121, 281 (2003) [hep-ph/0209016].

[62] G. Kramer and B. Lampe, Jet production rates at LEP and the scale of $\alpha_{s}$, Z. Phys. A 339, 189 (1991).

[63] S. Bethke et al., Workshop on precision measurements of $\alpha_{s}$, arXiv:1110.0016 [hep$\mathrm{ph}$. 
[64] D. d'Enterria et al., High-precision $\alpha_{s}$ measurements from LHC to FCC-ee, arXiv:1512.05194 [hep-ph].

[65] A. Pich, Review of $\alpha_{s}$ determinations, PoS ConfinementX , 022 (2012) [arXiv:1303.2262 [hep-ph]].

[66] J. Erler, Status of precision extractions of $\alpha_{s}$ and heavy quark masses, arXiv:1412.4435 [hep-ph].

[67] V. N. Gribov and L. N. Lipatov, Deep inelastic e p scattering in perturbation theory, Sov. J. Nucl. Phys. 15, 438 (1972) [Yad. Fiz. 15, 781 (1972)]; G. Altarelli and G. Parisi, Asymptotic freedom in parton language, Nucl. Phys. B 126, 298 (1977); Y. L. Dokshitzer, Calculation of the structure functions for deep inelastic scattering and $e^{+} e^{-}$annihilation by perturbation theory in quantum chromodynamics, Sov. Phys. JETP 46, 641 (1977) [Zh. Eksp. Teor. Fiz. 73, 1216 (1977)].

[68] E. Leader, A. V. Sidorov and D. B. Stamenov, Impact of CLAS and COMPASS data on polarized parton densities and higher twist, Phys. Rev. D 75, 074027 (2007) [hep-ph/0612360].

[69] D. J. Gross and C. H. Llewellyn Smith, High-energy neutrino-nucleon scattering, current algebra and partons, Nucl. Phys. B 14, 337 (1969).

[70] A. Deur et al., High precision determination of the $Q^{2}$ evolution of the Bjorken Sum, Phys. Rev. D 90, 012009 (2014) [arXiv:1405.7854 [nucl-ex]].

[71] G. Altarelli, R. D. Ball, S. Forte and G. Ridolfi, Determination of the Bjorken sum and strong coupling from polarized structure functions, Nucl. Phys. B 496, 337 (1997).

[72] J. H. Kim et al., A measurement of $\alpha_{s}\left(Q^{2}\right)$ from the Gross-Llewellyn Smith sum rule, Phys. Rev. Lett. 81, 3595 (1998) [hep-ex/9808015].

[73] F. J. Yndurain, Reconstruction of the deep inelastic structure functions from their moments, Phys. Lett. B 74, 68 (1978); J. Santiago and F. J. Yndurain, Calculation of electroproduction to NNLO and precision determination of $\alpha_{s}$, Nucl. Phys. B 563, 45 (1999) [hep-ph/9904344]; J. Santiago and F. J. Yndurain, Improved calculation of $F_{2}$ in electroproduction and $x F_{3}$ in neutrino scattering to NNLO and determination of $\alpha_{s}$, Nucl. Phys. B 611, 447 (2001) [hep-ph/0102247]; W. L. van 
Neerven and E. B. Zijlstra, Order $\alpha_{s}^{2}$ contributions to the deep inelastic Wilson coefficient, Phys. Lett. B 272, 127 (1991); E. B. Zijlstra and W. L. van Neerven, Order $\alpha_{s}^{2}$ QCD corrections to the deep inelastic proton structure functions $F_{2}$ and $F_{L}$, Nucl. Phys. B 383, 525 (1992); S. A. Larin, T. van Ritbergen and J. A. M. Vermaseren, The Next next-to-leading QCD approximation for non-singlet moments of deep inelastic structure functions, Nucl. Phys. B 427, 41 (1994); S. A. Larin, P. Nogueira, T. van Ritbergen and J. A. M. Vermaseren, The three-loop QCD calculation of the moments of deep inelastic structure functions, Nucl. Phys. B 492, 338 (1997) [hep-ph/9605317].

[74] J. Blumlein, H. Bottcher and A. Guffanti, Non-singlet QCD analysis of deep inelastic world data at $O\left(\alpha_{s}^{3}\right)$, Nucl. Phys. B 774, 182 (2007) [hep-ph/0607200]; S. Alekhin, J. Blumlein and S. Moch, Parton distribution functions and benchmark cross sections at NNLO, Phys. Rev. D 86, 054009 (2012) [arXiv:1202.2281 [hep-ph]]; S. Alekhin, J. Blmlein and S. O. Moch, ABM news and benchmarks, PoS DIS 2013, 039 (2013) [arXiv:1308.5166 [hep-ph]].

[75] P. Jimenez-Delgado, The role of the input scale in parton distribution analyses, Phys. Lett. B 714, 301 (2012) [arXiv:1206.4262 [hep-ph]].

[76] P. Jimenez-Delgado and E. Reya, Dynamical NNLO parton distributions, Phys. Rev. D 79, 074023 (2009) [arXiv:0810.4274 [hep-ph]]; P. Jimenez-Delgado and E. Reya, Delineating parton distributions and the strong coupling, Phys. Rev. D 89, 074049 (2014) [arXiv:1403.1852 [hep-ph]].

[77] R. D. Ball et al. [NNPDF Collaboration], Parton distributions for the LHC Run II, JHEP 1504, 040 (2015) [arXiv:1410.8849 [hep-ph]].

[78] A. Jakovc, A. Patks and P. Psfay, Non-Gaussian fixed points in fermionic field theories without auxiliary Bose-fields, Eur. Phys. J. C 75, 2 (2015) [arXiv:1406.3195 [hep-th]].

[79] P. A. Baikov, K. G. Chetyrkin and J. H. Kuhn, Order $\alpha^{4}(s)$ QCD corrections to $Z$ and $\tau$ decays, Phys. Rev. Lett. 101, 012002 (2008) [arXiv:0801.1821 [hep-ph]]; P. A. Baikov, K. G. Chetyrkin and J. H. Kuhn, Adler function, Bjorken sum rule, and the Crewther relation to order $\alpha_{s}^{4}$ in a general gauge theory, Phys. Rev. Lett. 104, 132004 (2010) [arXiv:1001.3606 [hep-ph]]. 
[80] E. Braaten, QCD predictions for the decay of the $\tau$ lepton, Phys. Rev. Lett. 60, 1606 (1988); E. Braaten, The perturbative QCD corrections to the ratio $R$ for $\tau$ decay, Phys. Rev. D 39, 1458 (1989); S. Narison and A. Pich, QCD formulation of the $\tau$ decay and determination of $\Lambda_{M S}$, Phys. Lett. B 211, 183 (1988); E. Braaten, S. Narison and A. Pich, QCD analysis of the $\tau$ hadronic width, Nucl. Phys. B 373, 581 (1992).

[81] F. Le Diberder and A. Pich, The perturbative QCD prediction to $R_{\tau}$ revisited, Phys. Lett. B 286, 147 (1992); F. Le Diberder and A. Pich, Testing QCD with $\tau$ decays, Phys. Lett. B 289, 165 (1992).

[82] G. Abbas, B. Ananthanarayan, I. Caprini and J. Fischer, Perturbative expansion of the QCD Adler function improved by renormalization-group summation and analytic continuation in the Borel plane, Phys. Rev. D 87, 014008 (2013) [arXiv:1211.4316 [hep-ph]].

[83] R. Barate et al. [ALEPH Collaboration], Measurement of the axial-vector $\tau$ spectral functions and determination of $\alpha_{s}\left(M_{\tau}^{2}\right)$ from hadronic $\tau$ decays, Eur. Phys. J. C 4, 409 (1998); S. Schael et al. [ALEPH Collaboration], Branching ratios and spectral functions of $\tau$ decays: Final ALEPH measurements and physics implications, Phys. Rept. 421, 191 (2005) [hep-ex/0506072].

[84] K. Ackerstaff et al. [OPAL Collaboration], Measurement of the strong coupling constant $\alpha_{s}$ and the vector and axial vector spectral functions in hadronic $\tau$ decays, Eur. Phys. J. C 7, 571 (1999) [hep-ex/9808019].

[85] T. Gehrmann, G. Luisoni and P. F. Monni, Power corrections in the dispersive model for a determination of the strong coupling constant from the thrust distribution, Eur. Phys. J. C 73, 2265 (2013) [arXiv:1210.6945 [hep-ph]].

[86] W. Braunschweig et al. [TASSO Collaboration], Global jet properties at $14-\mathrm{GeV}$ to 44-GeV center-of-mass energy in $e^{+} e^{-}$annihilation, Z. Phys. C 47, 187 (1990).

[87] D. Buskulic et al. [ALEPH Collaboration], Studies of QCD in $e^{+} e^{-} \rightarrow$ hadrons at $E_{c m}=130-\mathrm{GeV}$ and 136-GeV, Z. Phys. C 73, 409 (1997); A. Heister et al. [ALEPH Collaboration], Studies of QCD at $e^{+} e^{-}$centre-of-mass energies between $91-\mathrm{GeV}$ and 209-GeV, Eur. Phys. J. C 35, 457 (2004). 
[88] M. Acciarri et al. [L3 Collaboration], Study of the structure of hadronic events and determination of $\alpha_{s}$ at $\sqrt{s}=130-\mathrm{GeV}$ and 136-GeV, Phys. Lett. B 371, 137 (1996); M. Acciarri et al. [L3 Collaboration], QCD studies and determination of $\alpha_{s}$ in $e^{+} e^{-}$collisions at $\sqrt{s}=161-\mathrm{GeV}$ and 172-GeV, Phys. Lett. B 404, 390 (1997); M. Acciarri et al. [L3 Collaboration], QCD results from studies of hadronic events produced in $e^{+} e^{-}$annihilations at $\sqrt{s}=183-\mathrm{GeV}$, Phys. Lett. B 444, 569 (1998); P. Achard et al. [L3 Collaboration], Determination of $\alpha_{s}$ from hadronic event shapes in $e^{+} e^{-}$annihilation at $192-\mathrm{GeV} \leq \sqrt{s} \leq 208-\mathrm{GeV}$, Phys. Lett. B 536, 217 (2002); P. Achard et al. [L3 Collaboration], Studies of hadronic event structure in $e^{+} e^{-}$ annihilation from 30-GeV to 209-GeV with the L3 detector, Phys. Rept. 399, 71 (2004) [hep-ex/0406049].

[89] R. Abbate, M. Fickinger, A. H. Hoang, V. Mateu and I. W. Stewart, Thrust at $\mathrm{N}^{3} \mathrm{LL}$ with power corrections and a precision global fit for $\alpha_{s}\left(m_{Z}\right)$, Phys. Rev. D 83, 074021 (2011) [arXiv:1006.3080 [hep-ph]]; R. Abbate, M. Fickinger, A. H. Hoang, V. Mateu and I. W. Stewart, Precision thrust cumulant moments at $N^{3}$ LL, Phys. Rev. D 86, 094002 (2012) [arXiv:1204.5746 [hep-ph]].

[90] P. A. Movilla Fernandez et al. [JADE Collaboration], A study of event shapes and determinations of $\alpha_{s}$ using data of $e^{+} e^{-}$annihilations at $\sqrt{s}=22-\mathrm{GeV}$ to $44-\mathrm{GeV}$, Eur. Phys. J. C 1, 461 (1998) [hep-ex/9708034]; C. Pahl et al. [JADE Collaboration], Study of moments of event shapes and a determination of $\alpha_{s}$ using $e^{+} e^{-}$annihilation data from Jade, Eur. Phys. J. C 60, 181 (2009) [Eur. Phys. J. C 62, 451 (2009)] [arXiv:0810.2933 [hep-ex]].

[91] G. Abbiendi et al. [OPAL Collaboration], Measurement of event shape distributions and moments in $e^{+} e^{-} \rightarrow$ hadrons at $91-\mathrm{GeV}-209-\mathrm{GeV}$ and a determination of $\alpha_{s}$, Eur. Phys. J. C 40, 287 (2005) [hep-ex/0503051]; K. Ackerstaff et al. [OPAL Collaboration], QCD studies with $e^{+} e^{-}$annihilation data at 161-GeV, Z. Phys. C 75, 193 (1997).

[92] J. Abdallah et al. [DELPHI Collaboration], A Study of the energy evolution of event shape distributions and their means with the DELPHI detector at LEP, Eur. Phys. J. C 29, 285 (2003) [hep-ex/0307048]; J. Abdallah et al. [DELPHI Collaboration], The Measurement of $\alpha_{s}$ from event shapes with the DELPHI detector at the highest LEP energies, Eur. Phys. J. C 37, 1 (2004) [hep-ex/0406011]; P. Abreu et al. 
[DELPHI Collaboration], Energy dependence of event shapes and of $\alpha_{s}$ at LEP-2, Phys. Lett. B 456, 322 (1999).

[93] Y. K. Li et al. [AMY Collaboration], Multi-hadron event properties in $e^{+} e^{-}$annihilation at $\sqrt{s}=52 \mathrm{GeV}$ to 57-GeV, Phys. Rev. D 41, 2675 (1990).

[94] A. H. Hoang, D. W. Kolodrubetz, V. Mateu and I. W. Stewart, Precise determination of $\alpha_{s}$ from the $C$-parameter distribution, Phys. Rev. D 91, 094018 (2015) [arXiv:1501.04111 [hep-ph]].

[95] K. Abe et al. [SLD Collaboration], Measurement of $\alpha_{s}\left(M_{Z}^{2}\right)$ from hadronic event observables at the $Z_{0}$ resonance, Phys. Rev. D 51, 962 (1995) [hep-ex/9501003].

[96] P. Bolzoni, B. A. Kniehl and A. V. Kotikov, Average gluon and quark jet multiplicities at higher orders, Nucl. Phys. B 875, 18 (2013) [arXiv:1305.6017 [hep-ph]].

[97] V. Khachatryan et al. [CMS Collaboration], Constraints on parton distribution functions and extraction of the strong coupling constant from the inclusive jet cross section in $p p$ collisions at $\sqrt{s}=7 \mathrm{TeV}$, Eur. Phys. J. C 75, 288 (2015) [arXiv:1410.6765 [hep-ex]].

[98] A. D. Martin, W. J. Stirling, R. S. Thorne and G. Watt, Parton distributions for the LHC, Eur. Phys. J. C 63, 189 (2009) [arXiv:0901.0002 [hep-ph]]; A. D. Martin, W. J. Stirling, R. S. Thorne and G. Watt, Uncertainties on $\alpha_{s}$ in global PDF analyses and implications for predicted hadronic cross sections, Eur. Phys. J. C 64, 653 (2009) [arXiv:0905.3531 [hep-ph]]; R. S. Thorne, L. A. Harland-Lang, A. D. Martin and P. Motylinski, Updates of PDFs in the MSTW framework, PoS DIS 2014, 046 (2014) [arXiv:1407.4045 [hep-ph]].

[99] S. Aoki et al., Review of lattice results concerning low-energy particle physics, Eur. Phys. J. C 74, 2890 (2014) [arXiv:1310.8555 [hep-lat]].

[100] P. A. M. Dirac, The Lagrangian in quantum mechanics, Phys. Z. Sowjetunion 3, 64 (1933); R. P. Feynman, Space-time approach to nonrelativistic quantum mechanics, Rev. Mod. Phys. 20, 367 (1948).

[101] G. C. Wick, Properties of Bethe-Salpeter wave functions, Phys. Rev. 96, 1124 (1954). 
[102] N. Metropolis et al., J. Chem. Phys. 211087 (1953); Equations of state calculations by fast computing machines; W.K. Hastings, Monte Carlo sampling methods using Markov chains and their applications, Biometrika 57, 97 (1970).

[103] K. G. Wilson, Confinement of quarks, Phys. Rev. D 10, 2445 (1974).

[104] P. H. Ginsparg and K. G. Wilson, A remnant of chiral symmetry on the lattice, Phys. Rev. D 25, 2649 (1982).

[105] J. B. Kogut and L. Susskind, Hamiltonian formulation of Wilson's lattice gauge theories, Phys. Rev. D 11, 395 (1975).

[106] See e.g., G. P. Lepage, Lattice QCD for novices, hep-lat/0506036.

[107] See e.g. the reviews: V. Bernard and U. G. Meissner, Ann. Rev. Nucl. Part. Sci. 57, 33 (2007) doi:10.1146/annurev.nucl.56.080805.140449 [hep-ph/0611231]; S. Scherer, Prog. Part. Nucl. Phys. 64, 1 (2010) [arXiv:0908.3425 [hep-ph]].

[108] C. Michael, The running coupling from lattice gauge theory, Phys. Lett. B 283, 103 (1992) [hep-lat/9205010]; S. P. Booth et al. [UKQCD Collaboration], The running coupling from SU(3) lattice gauge theory, Phys. Lett. B 294, 385 (1992) [heplat/9209008].

[109] A. Bazavov, N. Brambilla, X. Garcia i Tormo, P. Petreczky, J. Soto and A. Vairo, Determination of $\alpha_{s}$ from the QCD static energy, Phys. Rev. D 86, 114031 (2012) [arXiv:1205.6155 [hep-ph]]; A. Bazavov, N. Brambilla, X. Garcia i Tormo, P. Petreczky, J. Soto and A. Vairo, Determination of $\alpha_{s}$ from the QCD static energy: An update, Phys. Rev. D 90, 074038 (2014) [arXiv:1407.8437 [hep-ph]].

[110] K. Jansen et al. [ETM Collaboration], $\Lambda_{\overline{M S}}$ from the static potential for QCD with $n_{f}=2$ dynamical quark flavors, JHEP 1201, 025 (2012) [arXiv:1110.6859 [hep-ph]]; F. Karbstein, A. Peters and M. Wagner, $\Lambda_{\overline{\mathrm{MS}}}^{\left(n_{f}=2\right)}$ from a momentum space analysis of the quark-antiquark static potential, JHEP 1409, 114 (2014) [arXiv:1407.7503 [hep-ph]].

[111] C. McNeile, C. T. H. Davies, E. Follana, K. Hornbostel and G. P. Lepage, Highprecision $\mathrm{c}$ and b masses, and QCD coupling from current-current correlators in lattice and continuum QCD, Phys. Rev. D 82, 034512 (2010) [arXiv:1004.4285 [heplat]]; B. Chakraborty et al., High-precision quark masses and QCD coupling from $n_{f}=4$ lattice QCD, Phys. Rev. D 91, 054508 (2015) [arXiv:1408.4169 [hep-lat]]. 
[112] E. Shintani, S. Aoki, H. Fukaya, S. Hashimoto, T. Kaneko, T. Onogi and N. Yamada, Strong coupling constant from vacuum polarization functions in three-flavor lattice QCD with dynamical overlap fermions, Phys. Rev. D 82, 074505 (2010) [Phys. Rev. D 89, 099903 (2014)] [arXiv:1002.0371 [hep-lat]].

[113] C. T. H. Davies et al. [HPQCD and UKQCD and MILC and Fermilab Lattice Collaborations], High precision lattice QCD confronts experiment, Phys. Rev. Lett. 92, 022001 (2004) [hep-lat/0304004]; Q. Mason et al. [HPQCD and UKQCD Collaborations], Accurate determinations of $\alpha_{s}$ from realistic lattice QCD, Phys. Rev. Lett. 95, 052002 (2005) [hep-lat/0503005]; C. T. H. Davies et al. [HPQCD Collaboration], Update: Accurate determinations of $\alpha_{s}$ from realistic lattice QCD, Phys. Rev. D 78, 114507 (2008) [arXiv:0807.1687 [hep-lat]].

[114] K. Maltman, D. Leinweber, P. Moran and A. Sternbeck, The realistic lattice determination of $\alpha_{s}\left(M_{Z}\right)$ revisited, Phys. Rev. D 78, 114504 (2008) [arXiv:0807.2020 [hep-lat]].

[115] A. Spitz et al. [TXL Collaboration], $\alpha_{s}$ from upsilon spectroscopy with dynamical Wilson fermions, Phys. Rev. D 60, 074502 (1999) [hep-lat/9906009].

[116] B. Blossier et al., The strong running coupling at $\tau$ and $Z_{0}$ mass scales from lattice QCD, Phys. Rev. Lett. 108, 262002 (2012) [arXiv:1201.5770 [hep-ph]]; Ghost-gluon coupling, power corrections and $\Lambda_{\overline{\mathrm{MS}}}$ from lattice QCD with a dynamical charm, Phys. Rev. D 85, 034503 (2012) [arXiv:1110.5829 [hep-lat]]; B. Blossier et al. [ETM Collaboration], High statistics determination of the strong coupling constant in Taylor scheme and its OPE Wilson coefficient from lattice QCD with a dynamical charm, Phys. Rev. D 89, 014507 (2014) [arXiv:1310.3763 [hep-ph]].

[117] M. Della Morte et al. [ALPHA Collaboration], Computation of the strong coupling in QCD with two dynamical flavors, Nucl. Phys. B 713, 378 (2005) [heplat/0411025]; P. Fritzsch, F. Knechtli, B. Leder, M. Marinkovic, S. Schaefer, R. Sommer and F. Virotta, The strange quark mass and Lambda parameter of two flavor QCD, Nucl. Phys. B 865, 397 (2012) [arXiv:1205.5380 [hep-lat]].

[118] R. Sommer and U. Wolff, Non-perturbative computation of the strong coupling constant on the lattice, Nucl. Part. Phys. Proc. 261-262, 155 (2015) [arXiv:1501.01861 [hep-lat]]. 
[119] N. Brambilla, X. Garcia i Tormo, J. Soto and A. Vairo, Extraction of $\alpha_{s}$ from radiative $\Upsilon(1 S)$ decays, Phys. Rev. D 75, 074014 (2007) [hep-ph/0702079].

[120] D. Besson et al. [CLEO Collaboration], Measurement of the direct photon momentum spectrum in $\Upsilon(1 S), \Upsilon(1 S)$, and $\Upsilon(1 S)$ decays, Phys. Rev. D 74, 012003 (2006) [hep-ex/0512061].

[121] S. J. Brodsky and G. F. de Teramond, Hadronic spectra and light-front wavefunctions in holographic QCD, Phys. Rev. Lett. 96, 201601 (2006) [hep-ph/0602252].

[122] G. F. de Teramond and S. J. Brodsky, Light-front holography: A First Approximation to QCD, Phys. Rev. Lett. 102, 081601 (2009) [arXiv:0809.4899 [hep-ph]]; G. F. de Teramond, H. G. Dosch and S. J. Brodsky, Kinematical and dynamical aspects of higher-spin bound-state equations in holographic QCD, Phys. Rev. D 87, 075005 (2013) [arXiv:1301.1651 [hep-ph]].

[123] P. A. M. Dirac, Forms of relativistic dynamics, Rev. Mod. Phys. 21, 392 (1949).

[124] S. J. Brodsky, G. F. de Teramond, H. G. Dosch and J. Erlich, Lightfront holographic QCD and emerging confinement, Phys. Rept. 584, 1 (2015) [arXiv:1407.8131 [hep-ph]].

[125] A. Deur, S. J. Brodsky and G. F. de Teramond, Connecting the hadron mass scale to the fundamental mass scale of quantum chromodynamics, Phys. Lett. B 750, 528 (2015) [arXiv:1409.5488 [hep-ph]].

[126] E. D. Bloom and F. J. Gilman, Scaling, duality, and the behavior of resonances in inelastic electron-proton scattering, Phys. Rev. Lett. 25, 1140 (1970); E. D. Bloom and F. J. Gilman, Scaling and the behavior of nucleon resonances in inelastic electron-nucleon scattering, Phys. Rev. D 4, 2901 (1971).

[127] For a review, see e.g. W. Melnitchouk, R. Ent and C. Keppel, Quark-hadron duality in electron scattering, Phys. Rept. 406, 127 (2005) [hep-ph/0501217].

[128] J.-L. Kneur and A. Neveu, $\Lambda_{\mathrm{MS}}^{\mathrm{QCD}}$ from renormalization group optimized perturbation, Phys. Rev. D 85, 014005 (2012) [arXiv:1108.3501 [hep-ph]]; J. L. Kneur and A. Neveu, $\alpha_{s}$ from $F_{\pi}$ and renormalization group optimized perturbation theory, Phys. Rev. D 88, 074025 (2013) [arXiv:1305.6910 [hep-ph]]. 
[129] P. L. Anthony et al. [SLAC E158 Collaboration], Precision measurement of the weak mixing angle in Moller scattering, Phys. Rev. Lett. 95, 081601 (2005) [hepex/0504049]; D. Androic et al. [Qweak Collaboration], First determination of the weak charge of the proton, Phys. Rev. Lett. 111, 141803 (2013) [arXiv:1307.5275 [nucl-ex]]; D. Wang et al. [PVDIS Collaboration], Measurement of parity violation in electron-quark scattering, Nature 506, 67 (2014).

[130] P. Langacker and N. Polonsky, The strong coupling, unification, and recent data, Phys. Rev. D 52, 3081 (1995) [hep-ph/9503214].

[131] L. J. Hall and Y. Nomura, A complete theory of grand unification in fivedimensions, Phys. Rev. D 66, 075004 (2002) [hep-ph/0205067].

[132] D. d'Enterria and R. Perez-Ramos, Extraction of $\alpha_{s}$ from the energy evolution of jet fragmentation functions at low $z$, arXiv:1408.2865 [hep-ph]; R. Perez-Ramos and D. d'Enterria, Determination of $\alpha_{s}$ at $\mathrm{NLO}^{*}+$ NNLL from a global fit of the low- $z$ parton-to-hadron fragmentation functions in $e^{+} e^{-}$and DIS collisions, EPJ Web Conf. 90, 04001 (2015).

[133] S. J. Brodsky, D. S. Hwang and I. Schmidt, Final state interactions and single spin asymmetries in semi-inclusive deep inelastic scattering, Phys. Lett. B 530, 99 (2002) [hep-ph/0201296].

[134] S. J. Brodsky, A. H. Hoang, J. H. Kuhn and T. Teubner, Angular distributions of massive quarks and leptons close to threshold, Phys. Lett. B 359, 355 (1995) [hep-ph/9508274].

[135] See e.g. H. Pagels, Dynamical chiral symmetry breaking in quantum chromodynamics, Phys. Rev. D 19, 3080 (1979); K. Higashijima, Dynamical chiral symmetry breaking, Phys. Rev. D 29, 1228 (1984).

[136] T. Barnes, F. E. Close and S. Monaghan, Hyperfine splittings of bag model gluonia, Nucl. Phys. B 198, 380 (1982).

[137] J. Stern and G. Clement, Quarks, gluons, pions and the Gottfried sum rule, Phys. Lett. B 264, 426 (1991).

[138] H. Contopanagos, L. Alvero and G. F. Sterman, Principal value resummation for dilepton production, hep-ph/9408393. 
[139] S. Catani, M. L. Mangano, P. Nason and L. Trentadue, The resummation of soft gluons in hadronic collisions, Nucl. Phys. B 478, 273 (1996) [hep-ph/9604351].

[140] \%bibitemBadelek:1996ap B. Badelek, J. Kwiecinski and A. Stasto, A Model for $\mathrm{F}(\mathrm{L})$ and $\mathrm{R}=\mathrm{F}(\mathrm{L}) / \mathrm{F}(\mathrm{T})$ at low $\mathrm{x}$ and low $\mathrm{Q}^{* *} 2$, Z. Phys. C 74, 297 (1997) [hep-ph/9603230].

[141] K. A. Milton, I. L. Solovtsov and O. P. Solovtsova, An Analytic method of describing R-related quantities in QCD, Mod. Phys. Lett. A 21, 1355 (2006) [hep$\mathrm{ph} / 0512209]$.

[142] A. Courtoy, S. Scopetta and V. Vento, Non-perturbative momentum dependence of the coupling constant and hadronic models, Eur. Phys. J. A 47, 49 (2011) [arXiv:1102.1599 [hep-ph]].

[143] M. R. Pennington, Strong Coupling Continuum QCD, AIP Conf. Proc. 1343, 63 (2011) [arXiv:1104.2522 [nucl-th]].

[144] N. G. Stefanis, The Physics of exclusive reactions in QCD: Theory and phenomenology, Eur. Phys. J. direct C 7, 1 (1999) [hep-ph/9911375].

[145] D. V. Shirkov, On the QCD coupling behavior in the infrared region, Theor. Math. Phys. 132, 1309 (2002) [Teor. Mat. Fiz. 132, 484 (2002)] [hep-ph/0208082].

[146] J. A. Gracey, "Two loop QCD vertices at the symmetric point," Phys. Rev. D 84, 085011 (2011) [arXiv:1108.4806 [hep-ph]]; "Momentum subtraction and the $R$ ratio," Phys. Rev. D 90, 094026 (2014) [arXiv:1410.6715 [hep-ph]].

[147] Aspects of $\mathrm{SU}(\mathrm{N}(\mathrm{c}))$ gauge theories in the limit of small number of colors S. J. Brodsky, P. Huet, Phys. Lett. B 417145 (1998). [hep-ph/9707543]

[148] See e.g. T. J. Rivers, Path Integral Methods in Quantum Field Theory, Cambridge Monographs on Mathematical Physics. Cambridge University Press (1988).

[149] For a proper comparison, the coupling in Fig. 4.1 should be expressed in the $R$ scheme, or alternatively one should consider the potential on the light-front, see the discussion in Section 4.2. The argument remains true for these more proper comparisons. It also remains true a fortiori if the decoupling solution of $\alpha_{s}$ (see Section 4.4.3) is realized in Nature rather than the scaling solution shown in Fig. 4.1 . 
[150] G. S. Bali, T. Dussel, T. Lippert, H. Neff, Z. Prkacin and K. Schilling, String breaking, Nucl. Phys. Proc. Suppl. 153, 9 (2006) [hep-lat/0512018].

[151] B. Bolder, T. Struckmann, G. S. Bali, N. Eicker, T. Lippert, B. Orth, K. Schilling and P. Ueberholz, A High precision study of the Q anti-Q potential from Wilson loops in the regime of string breaking, Phys. Rev. D 63, 074504 (2001) [heplat/0005018].

[152] Y. L. Dokshitzer, V. A. Khoze and S. I. Troian, Specific features of heavy quark production. LPHD approach to heavy particle spectra, Phys. Rev. D 53, 89 (1996) [hep-ph/9506425]; Y. L. Dokshitzer, A. Lucenti, G. Marchesini and G. P. Salam, On the universality of the Milan factor for 1/Q power corrections to jet shapes, JHEP 9805, 003 (1998) [hep-ph/9802381].

[153] A. I. Shoshi, F. D. Steffen, H. G. Dosch and H. J. Pirner, Confining QCD strings, Casimir scaling, and a Euclidean approach to high-energy scattering, Phys. Rev. D 68, 074004 (2003) [hep-ph/0211287].

[154] V. Gribov, QCD at large and short distances (annotated version), Eur. Phys. J. C 10, 71 (1999) [hep-ph/9807224].

[155] V. N. Gribov, Quantization of Nonabelian Gauge Theories, Nucl. Phys. B 139, 1 (1978).

[156] A. Cucchieri, T. Mendes and D. Zwanziger, SU(2) running coupling constant and confinement in minimal coulomb and Landau gauges, Nucl. Phys. Proc. Suppl. 106, 697 (2002) [hep-lat/0110188].

[157] A. P. Szczepaniak and E. S. Swanson, Coulomb gauge QCD, confinement, and the constituent representation, Phys. Rev. D 65, 025012 (2002) [hep-ph/0107078].

[158] A. Nakamura and T. Saito, Color confinement in Coulomb gauge QCD, Prog. Theor. Phys. 115, 189 (2006) [hep-lat/0512042]; A. Nakamura and T. Saito, QCD color interactions between two quarks, Phys. Lett. B 621, 171 (2005) [hep-lat/0512043]; Y. Nakagawa, A. Voigt, E.-M. Ilgenfritz, M. Muller-Preussker, A. Nakamura, T. Saito, A. Sternbeck and H. Toki, Coulomb-gauge ghost and gluon propagators in SU(3) lattice Yang-Mills theory, Phys. Rev. D 79, 114504 (2009) [arXiv:0902.4321 [hep-lat]]; Y. Nakagawa, A. Nakamura, T. Saito and H. Toki, 
Spectral sum for the color-Coulomb potential in SU(3) Coulomb gauge lattice YangMills theory, Phys. Rev. D 81, 054509 (2010) [arXiv:1003.4792 [hep-lat]]; Y. Nakagawa, A. Nakamura, T. Saito and H. Toki, Scaling study of the gluon propagator in Coulomb gauge QCD on isotropic and anisotropic lattices, Phys. Rev. D 83, 114503 (2011) [arXiv:1105.6185 [hep-lat]].

[159] A. Cucchieri and D. Zwanziger, Gluon propagator and confinement scenario in Coulomb gauge, Nucl. Phys. Proc. Suppl. 119, 727 (2003) [hep-lat/0209068]; J. Greensite and S. Olejnik, Coulomb energy, vortices, and confinement, Phys. Rev. D 67, 094503 (2003) [hep-lat/0302018].

[160] A. I. Sanda, A Nonperturbative Determination of $\alpha\left(q^{2}\right)$ and Its Experimental Implications, Phys. Rev. Lett. 42, 1658 (1979).

[161] T. Banks and A. Zaks, On the Phase Structure of Vector-Like Gauge Theories with Massless Fermions, Nucl. Phys. B 196, 189 (1982).

[162] S. J. Brodsky, G. F. de Teramond and A. Deur, Nonperturbative QCD Coupling and its $\beta$-function from Light-Front Holography, Phys. Rev. D 81, 096010 (2010) [arXiv:1002.3948 [hep-ph]].

[163] S. J. Brodsky, New perspectives for QCD: The Novel effects of final state interactions and near conformal effective couplings, Fizika B 13, 91 (2004) [hep$\mathrm{ph} / 0310289]$.

[164] B. A. Arbuzov, Infrared non-perturbative QCD running coupling from Bogolubov approach, Phys. Lett. B 656, 67 (2007) [hep-ph/0703237 [HEP-PH]]; B. A. Arbuzov and I. V. Zaitsev, Elimination of the Landau pole in QCD with the spontaneously generated anomalous three-gluon interaction, arXiv:1303.0622 [hep-th].

[165] P. Boucaud, F. De Soto, A. Le Yaouanc, J. P. Leroy, J. Micheli, H. Moutarde, O. Pene and J. Rodriguez-Quintero, The Strong coupling constant at small momentum as an instanton detector, JHEP 0304, 005 (2003) [hep-ph/0212192]; P. Boucaud, F. De Soto, A. Le Yaouanc, J. P. Leroy, J. Micheli, O. Pene and J. RodriguezQuintero, Modified instanton profile effects from lattice Green functions, Phys. Rev. D 70, 114503 (2004) [hep-ph/0312332].

[166] M. Baldicchi, A. V. Nesterenko, G. M. Prosperi, D. V. Shirkov and C. Simolo, Bound state approach to the QCD coupling at low energy scales, Phys. Rev. Lett. 
99, 242001 (2007) [arXiv:0705.0329 [hep-ph]]; M. Baldicchi, A. V. Nesterenko, G. M. Prosperi and C. Simolo, QCD coupling below $1 \mathrm{GeV}$ from quarkonium spectrum, Phys. Rev. D 77, 034013 (2008) [arXiv:0705.1695 [hep-ph]].

[167] M. Burkardt, Transverse force on quarks in deep-inelastic scattering, Phys. Rev. D 88, 114502 (2013) [arXiv:0810.3589 [hep-ph]].

[168] Y. L. Dokshitzer, G. Marchesini and B. R. Webber, Dispersive approach to power behaved contributions in QCD hard processes, Nucl. Phys. B 469, 93 (1996) [hep$\mathrm{ph} / 9512336]$.

[169] Y. L. Dokshitzer and B. R. Webber, Calculation of power corrections to hadronic event shapes, Phys. Lett. B 352, 451 (1995) [hep-ph/9504219].

[170] S. B. Gerasimov, A Sum rule for magnetic moments and the damping of the nucleon magnetic moment in nuclei, Sov. J. Nucl. Phys. 2, 430 (1966) [Yad. Fiz. 2, 598 (1965)]; S. D. Drell and A. C. Hearn, Exact Sum Rule for Nucleon Magnetic Moments, Phys. Rev. Lett. 16, 908 (1966).

[171] P. P. L. Anthony et al. [E142 Collaboration], Determination of the neutron spin structure function., Phys. Rev. Lett. 71, 959 (1993); P. L. Anthony et al. [E142 Collaboration], Deep inelastic scattering of polarized electrons by polarized He-3 and the study of the neutron spin structure, Phys. Rev. D 54, 6620 (1996) [hepex/9610007].

[172] D. Adams et al. [Spin Muon (SMC) Collaboration], Measurement of the spin dependent structure function $g_{1}(x)$ of the proton, Phys. Lett. B 329, 399 (1994) [hep-ph/9404270];D. Adams et al. [Spin Muon (SMC) Collaboration], Spin asymmetry in muon - proton deep inelastic scattering on a transversely polarized target, Phys. Lett. B 336, 125 (1994) [hep-ex/9408001]; D. Adams et al. [Spin Muon Collaboration], A New measurement of the spin dependent structure function g1(x) of the deuteron, Phys. Lett. B 357, 248 (1995); D. Adams et al. [Spin Muon (SMC) Collaboration], The Spin dependent structure function $\mathrm{g} 1(\mathrm{x})$ of the deuteron from polarized deep inelastic muon scattering, Phys. Lett. B 396, 338 (1997); D. Adams et al. [Spin Muon (SMC) Collaboration], Spin structure of the proton from polarized inclusive deep inelastic muon - proton scattering, Phys. Rev. D 56, 5330 (1997) [hep-ex/9702005]. 
[173] K. Abe et al. [E143 Collaboration], Precision measurement of the proton spin structure function g1(p), Phys. Rev. Lett. 74, 346 (1995); K. Abe et al. [E143 Collaboration], Precision measurement of the deuteron spin structure function g1(d), Phys. Rev. Lett. 75, 25 (1995); K. Abe et al. [E143 Collaboration], Measurements of the proton and deuteron spin structure function g2 and asymmetry A2, Phys. Rev. Lett. 76, 587 (1996) [hep-ex/9511013]; K. Abe et al. [E143 Collaboration], Measurements of the $\mathrm{Q}^{* *} 2$ dependence of the proton and deuteron spin structure functions g1(p) and g1(d), Phys. Lett. B 364, 61 (1995) [hep-ex/9511015]; K. Abe et al. [E143 Collaboration], Measurements of the proton and deuteron spin structure functions g(1) and g(2), Phys. Rev. D 58, 112003 (1998) [hep-ph/9802357].

[174] K. Abe et al. [E154 Collaboration], Precision determination of the neutron spin structure function g1(n), Phys. Rev. Lett. 79, 26 (1997) [hep-ex/9705012]; K. Abe et al. [E154 Collaboration], Measurement of the neutron spin structure function g2(n) and asymmetry A2(n), Phys. Lett. B 404, 377 (1997) [hep-ex/9705017]; K. Abe et al. [E154 Collaboration], Next-to-leading order QCD analysis of polarized deep inelastic scattering data, Phys. Lett. B 405, 180 (1997) [hep-ph/9705344].

[175] K. Ackerstaff et al. [HERMES Collaboration], Measurement of the neutron spin structure function g1(n) with a polarized He-3 internal target, Phys. Lett. B 404, 383 (1997) [hep-ex/9703005]; K. Ackerstaff et al. [HERMES Collaboration], Determination of the deep inelastic contribution to the generalized Gerasimov-DrellHearn integral for the proton and neutron, Phys. Lett. B 444, 531 (1998) [hepex/9809015]; A. Airapetian et al. [HERMES Collaboration], Measurement of the proton spin structure function $\mathrm{g} 1(\mathrm{p})$ with a pure hydrogen target, Phys. Lett. B 442, 484 (1998) [hep-ex/9807015]; A. Airapetian et al. [HERMES Collaboration], Evidence for quark hadron duality in the proton spin asymmetry A(1), Phys. Rev. Lett. 90, 092002 (2003) [hep-ex/0209018]; A. Airapetian et al. [HERMES Collaboration], Precise determination of the spin structure function $\mathrm{g}(1)$ of the proton, deuteron and neutron, Phys. Rev. D 75, 012007 (2007) [hep-ex/0609039].

[176] P. L. Anthony et al. [E155 Collaboration], Measurement of the proton and deuteron spin structure functions g(2) and asymmetry A(2), Phys. Lett. B 458, 529 (1999) [hep-ex/9901006]; P. L. Anthony et al. [E155 Collaboration], Measurement of the deuteron spin structure function $\mathrm{g} 1(\mathrm{~d})(\mathrm{x})$ for $1-(\mathrm{GeV} / \mathrm{c})^{* *} 2$; $\mathrm{Q}^{* *} 2$; $40-(\mathrm{GeV} / \mathrm{c})^{* *} 2$, Phys. Lett. B 463, 339 (1999) [hep-ex/9904002]; P. L. Anthony et al. [E155 Collaboration], Measurements of the $\mathrm{Q}^{* * 2} 2$ dependence of the proton and neutron 
spin structure functions $\mathrm{g}(1)^{* *} \mathrm{p}$ and $\mathrm{g}(1)^{* *} \mathrm{n}$, Phys. Lett. B 493, 19 (2000) [hepph/0007248];P. L. Anthony et al. [E155 Collaboration], Precision measurement of the proton and deuteron spin structure functions $\mathrm{g}(2)$ and asymmetries A(2), Phys. Lett. B 553, 18 (2003) [hep-ex/0204028].

[177] A. Deur et al., Experimental determination of the evolution of the Bjorken integral at low $Q^{2}$, Phys. Rev. Lett. 93, 212001 (2004) [hep-ex/0407007]; A. Deur et al., Experimental study of isovector spin sum rules, Phys. Rev. D 78, 032001 (2008) [arXiv:0802.3198 [nucl-ex]],

[178] V. Y. Alexakhin et al. [COMPASS Collaboration], The Deuteron Spin-dependent Structure Function g1(d) and its First Moment, Phys. Lett. B 647, 8 (2007) [hepex/0609038]; M. G. Alekseev et al. [COMPASS Collaboration], The Spin-dependent Structure Function of the Proton $g_{1}^{p}$ and a Test of the Bjorken Sum Rule, Phys. Lett. B 690, 466 (2010) [arXiv:1001.4654 [hep-ex]]; C. Adolph et al. [COMPASS Collaboration], The spin structure function $g_{1}^{\mathrm{p}}$ of the proton and a test of the Bjorken sum rule, Phys. Lett. B 753, 18 (2016) [arXiv:1503.08935 [hep-ex]].

[179] V. D. Burkert, Comment on the generalized Gerasimov-Drell-Hearn sum rule in chiral perturbation theory, Phys. Rev. D 63, 097904 (2001) [nucl-th/0004001].

[180] See e.g. F. Bloch and A. Nordsieck, Note on the Radiation Field of the electron, Phys. Rev. 52, 54 (1937); T. Kinoshita, Mass singularities of Feynman amplitudes, J. Math. Phys. 3, 650 (1962); T. D. Lee and M. Nauenberg, Degenerate Systems and Mass Singularities, Phys. Rev. 133, B1549 (1964).

[181] M. Anselmino, B. L. Ioffe and E. Leader, On Possible Resolutions of the Spin Crisis in the Parton Model, Sov. J. Nucl. Phys. 49 (1989) 136 [Yad. Fiz. 49 (1989) 214]; D. Drechsel, S. S. Kamalov and L. Tiator, The GDH sum rule and related integrals, Phys. Rev. D 63, 114010 (2001) [hep-ph/0008306]; X. D. Ji and J. Osborne, Generalized sum rules for spin dependent structure functions of the nucleon, J. Phys. G 27, 127 (2001) [hep-ph/9905410].

[182] A. Deur, V. Burkert, J. P. Chen and W. Korsch, Experimental determination of the effective strong coupling constant, Phys. Lett. B 650, 244 (2007) [hep-ph/0509113]; A. Deur, V. Burkert, J. P. Chen and W. Korsch, Determination of the effective strong coupling constant alpha(s,g(1))(Q**2) from CLAS spin structure function data, Phys. Lett. B 665, 349 (2008) [arXiv:0803.4119 [hep-ph]]. 
[183] G. Grunberg, On power corrections in the dispersive approach, JHEP 9811, 006 (1998) [hep-ph/9807494]; E. Gardi and G. Grunberg, Power corrections in the single dressed gluon approximation: The Average thrust as a case study, JHEP 9911, 016 (1999) [hep-ph/9908458]; E. Gardi and G. Grunberg, A Dispersive approach to Sudakov resummation, Nucl. Phys. B 794, 61 (2008) [arXiv:0709.2877 [hep-ph]].

[184] G. Grunberg, Infrared finite coupling in Sudakov resummation, hep-ph/0601140.

[185] J. Botts and G. F. Sterman, Hard Elastic Scattering in QCD: Leading Behavior, Nucl. Phys. B 325, 62 (1989); H. n. Li and G. F. Sterman, The Perturbative pion form-factor with Sudakov suppression, Nucl. Phys. B 381, 129 (1992).

[186] V. de Alfaro, S. Fubini and G. Furlan, Conformal invariance in quantum mechanics, Nuovo Cim. A 34, 569 (1976).

[187] S. J. Brodsky, G. F. De Teramond and H. G. Dosch, Threefold complementary approach to holographic QCD, Phys. Lett. B 729, 3 (2014) [arXiv:1302.4105 [hepth]].

[188] V. P. Akulov and A. I. Pashnev, Quantum superconformal model in $(1,2)$ space, Theor. Math. Phys. 56, 862 (1983) [Teor. Mat. Fiz. 56, 344 (1983)].

[189] S. Fubini and E. Rabinovici, Superconformal quantum mechanics, Nucl. Phys. B 245, 17 (1984).

[190] G. F. de Teramond, H. G. Dosch and S. J. Brodsky, Baryon spectrum from superconformal quantum mechanics and its light-front holographic embedding, Phys. Rev. D 91, 045040 (2015) [arXiv:1411.5243 [hep-ph]].

[191] H. G. Dosch, G. F. de Teramond and S. J. Brodsky, Superconformal baryonmeson symmetry and light-front holographic QCD, Phys. Rev. D 91, 085016 (2015) [arXiv:1501.00959 [hep-th]].

[192] S. J. Brodsky, G. F. de Teramond, H. G. Dosch and C. Lorce, Universal effective hadron dynamics from superconformal algebra, arXiv:1604.06746 [hep-ph].

[193] H. G. Dosch, G. F. de Teramond and S. J. Brodsky, Supersymmetry across the light and heavy-light hadronic spectrum, Phys. Rev. D 92, 074010 (2015) [arXiv:1504.05112 [hep-ph]]. 
[194] Hadronic supersymmetry was introduced by Miyazawa. See, H. Miyazawa, Baryon number changing currents, Prog. Theor. Phys. 36, 1266 (1966).

[195] S. Catto and F. Gursey, Algebraic treatment of effective supersymmetry, Nuovo Cim. A 86, 201 (1985).

[196] D. B. Lichtenberg, Whither hadron supersymmetry?, arXiv:hep-ph/9912280.

[197] A. P. Trawinski, S. D. Glazek, S. J. Brodsky, G. F. de Teramond and H. G. Dosch, Effective confining potentials for QCD, Phys. Rev. D 90, 074017 (2014) [arXiv:1403.5651 [hep-ph]].

[198] A. Karch, E. Katz, D. T. Son and M. A. Stephanov, Linear confinement and AdS/QCD, Phys. Rev. D 74, 015005 (2006) [hep-ph/0602229].

[199] I. C. Cloet and C. D. Roberts, Explanation and Prediction of Observables using Continuum Strong QCD, Prog. Part. Nucl. Phys. 77, 1 (2014) [arXiv:1310.2651 [nucl-th]].

[200] E. Eichten, K. Gottfried, T. Kinoshita, J. B. Kogut, K. D. Lane and T. M. Yan, The Spectrum of Charmonium, Phys. Rev. Lett. 34, 369 (1975) [Phys. Rev. Lett. 36, 1276 (1976)]; E. Eichten, K. Gottfried, T. Kinoshita, K. D. Lane and T. M. Yan, Charmonium: The Model, Phys. Rev. D 17, 3090 (1978) [Phys. Rev. D 21, 313 (1980)]; E. J. Eichten, K. Lane and C. Quigg, B meson gateways to missing charmonium levels, Phys. Rev. Lett. 89, 162002 (2002) [hep-ph/0206018].

[201] W. Celmaster and F. S. Henyey, The Quark - Anti-quark Interaction at All Momentum Transfers, Phys. Rev. D 18, 1688 (1978); R. Levine and Y. Tomozawa, An Effective Potential for Heavy Quark - Anti-quark Bound Systems, Phys. Rev. D 19, 1572 (1979); R. Y. Levine and Y. Tomozawa, Characteristics Of Expected Quark Anti-quark Resonances Based On The Quantum Chromodynamic Potential, Phys. Rev. D 21, 840 (1980); W. Buchmuller, G. Grunberg and S. H. H. Tye, The Regge Slope and the Lambda Parameter in QCD: An Empirical Approach via Quarkonia, Phys. Rev. Lett. 45, 103 (1980) [Phys. Rev. Lett. 45, 587 (1980)]; W. Buchmuller and S. H. H. Tye, Quarkonia and Quantum Chromodynamics, Phys. Rev. D 24, 132 (1981);

[202] D. P. Stanley and D. Robson, Nonperturbative Potential Model for Light and Heavy Quark anti-Quark Systems, Phys. Rev. D 21, 3180 (1980); D. P. Stanley 
and D. Robson, Do Quarks Interact Pairwise And Satisfy The Color Hypothesis?, Phys. Rev. Lett. 45, 235 (1980); W. Kwong, J. L. Rosner and C. Quigg, Heavy Quark Systems, Ann. Rev. Nucl. Part. Sci. 37, 325 (1987); D. Ebert, R. N. Faustov and V. O. Galkin, Quark - anti-quark potential with retardation and radiative contributions and the heavy quarkonium mass spectra, Phys. Rev. D 62, 034014 (2000) [hep-ph/9911283]; L. P. Fulcher, Perturbative QCD, a universal QCD scale, long range spin orbit potential, and the properties of heavy quarkonia, Phys. Rev. D 44, 2079 (1991); S. Jacobs, M. G. Olsson and C. Suchyta, III, Comparing the Schrodinger and Spinless Salpeter Equations for Heavy Quark Bound States, Phys. Rev. D 33, 3338 (1986) [Phys. Rev. D 34, 3536 (1986)]; T. J. Allen, M. G. Olsson and S. Veseli, From scalar to string confinement, Phys. Rev. D 62, 094021 (2000) [hep-ph/0001227].

[203] Y. Nambu, p. 269 in Proc. Intern. Conf. on Symmetries and Quark Models, ed. R. Chand, Wayne State Univ., 1969 (Gordon and Breach, NY 1970); Y. Nambu, Strings, Monopoles and Gauge Fields, Phys. Rev. D 10, 4262 (1974); L. Susskind, Harmonic-oscillator analogy for the veneziano model, Phys. Rev. Lett. 23, 545 (1969); L. Susskind, Dual symmetric theory of hadrons. 1., Nuovo Cim. A 69, 457 (1970); G. Frye, C. W. Lee and L. Susskind, Dual-symmetric theory of hadrons. 2. baryons, Nuovo Cim. A 69, 497 (1970) [Nuovo Cim. A 3, 497 (1970)]; D. B. Fairlie and H. B. Nielsen, An analog model for ksv theory, Nucl. Phys. B 20, 637 (1970).

[204] J. Greensite, An introduction to the confinement problem. Lecture Notes in Physics, 821. Springer, Heidelberg, 2011.

[205] N. Brambilla and A. Vairo, Quark confinement and the hadron spectrum, In *Newport News 1998, Strong interactions at low and intermediate energies* 151-220 [hep-ph/9904330].

[206] see for a review G. S. Bali, QCD forces and heavy quark bound states, Phys. Rept. 343, 1 (2001) [hep-ph/0001312], and for specific publications:

G. S. Bali and K. Schilling, Running coupling and the Lambda parameter from SU(3) lattice simulations, Phys. Rev. D 47, 661 (1993) [hep-lat/9208028]; G. S. Bali, K. Schilling and C. Schlichter, Observing long color flux tubes in SU(2) lattice gauge theory, Phys. Rev. D 51, 5165 (1995) [hep-lat/9409005]; G. S. Bali, Are there short distance nonperturbative contributions to the QCD static potential?, Phys. Lett. 
B 460, 170 (1999) [hep-ph/9905387]; G. S. Bali et al. [TXL and T(X)L Collaborations], Static potentials and glueball masses from QCD simulations with Wilson sea quarks, Phys. Rev. D 62, 054503 (2000) [hep-lat/0003012]; C. W. Bernard et al., The QCD spectrum with three quark flavors, Phys. Rev. D 64, 054506 (2001) [heplat/0104002]; S. Necco and R. Sommer, The N(f) = 0 heavy quark potential from short to intermediate distances, Nucl. Phys. B 622, 328 (2002) [hep-lat/0108008]; T. T. Takahashi, H. Suganuma, Y. Nemoto and H. Matsufuru, Detailed analysis of the three quark potential in SU(3) lattice QCD, Phys. Rev. D 65, 114509 (2002) [hep-lat/0204011]; S. Aoki et al. [JLQCD Collaboration], Light hadron spectroscopy with two flavors of $\mathrm{O}(\mathrm{a})$ improved dynamical quarks, Phys. Rev. D 68, 054502 (2003) [hep-lat/0212039].

[207] D. Zwanziger, Covariant Quantization of Gauge Fields Without Gribov Ambiguity, Nucl. Phys. B 192, 259 (1981); D. Zwanziger, Nonperturbative Modification of the Faddeev-popov Formula and Banishment of the Naive Vacuum, Nucl. Phys. B 209, 336 (1982); D. Zwanziger, Local and Renormalizable Action From the Gribov Horizon, Nucl. Phys. B 323, 513 (1989); D. Zwanziger, Vanishing of zero momentum lattice gluon propagator and color confinement, Nucl. Phys. B 364, 127 (1991); D. Zwanziger, Renormalizability of the critical limit of lattice gauge theory by BRS invariance, Nucl. Phys. B 399, 477 (1993);

[208] J. A. Gracey, The One loop anti-MS static potential in the Gribov-Zwanziger Lagrangian, JHEP 1002, 009 (2010) [arXiv:0909.3411 [hep-th]]; J. A. Gracey, Loop calculations in the three dimensional Gribov-Zwanziger Lagrangian, Eur. Phys. J. C 70, 451 (2010) [arXiv:1010.1104 [hep-th]].

[209] C. Feuchter and H. Reinhardt, Variational solution of the Yang-Mills Schrodinger equation in Coulomb gauge, Phys. Rev. D 70, 105021 (2004) [hep-th/0408236].

[210] D. Epple, H. Reinhardt and W. Schleifenbaum, Confining Solution of the DysonSchwinger Equations in Coulomb Gauge, Phys. Rev. D 75, 045011 (2007) [hepth/0612241].

[211] U. Ellwanger, M. Hirsch and A. Weber, The Heavy quark potential from Wilson's exact renormalization group, Eur. Phys. J. C 1, 563 (1998) [hep-ph/9606468].

[212] Y. A. Simonov, Perturbative theory in the nonperturbative QCD vacuum, Phys. Atom. Nucl. 58, 107 (1995) [Yad. Fiz. 58, 113 (1995)] [hep-ph/9311247]; Y. A. Si- 
monov, Asymptotic freedom and IR freezing in QCD: the role of gluon paramagnetism, Phys. Atom. Nucl. 74, 1223 (2011) [arXiv:1011.5386 [hep-ph]]. See also A. M. Badalian and Y. A. Simonov, Freezing of alpha $(\mathrm{s})\left(\mathrm{Q}^{* *} 2\right)$ in e+ e- annihilation, Phys. Atom. Nucl. 60, 630 (1997) [Yad. Fiz. 60, 714 (1997)]. A. M. Badalian, Running coupling constant in lattice QCD and nonperturbative effects, Phys. Atom. Nucl. 60, 1003 (1997) [Yad. Fiz. 60, 1122 (1997)].

[213] S. Godfrey and N. Isgur, Mesons in a Relativized Quark Model with Chromodynamics, Phys. Rev. D 32, 189 (1985).

[214] T. Zhang and R. Koniuk, A Relativistic quark model, Phys. Lett. B 244, 493 (1990). T. Zhang and R. Koniuk, Heavy quark mesons in a relativistic quark model, Phys. Rev. D 43, 1688 (1991); T. Zhang and R. Koniuk, The pi rho mass splitting, Phys. Lett. B 261, 311 (1991).

[215] A. M. Badalian and A. I. Veselov, The Static force in background perturbation theory, Phys. Atom. Nucl. 68, 582 (2005) [Yad. Fiz. 68, 612 (2005)] [hep-ph/0407082].

[216] V. V. Andreev, QCD coupling constant below $1 \mathrm{GeV}$ in the poincare-covariant model Phys. Part. Nucl. Lett. 4 (2011) 347; V. V. Andreev, Nonperturbative region of effective strong coupling, arXiv:1305.4266 [hep-ph].

[217] M. Baldicchi and G. M. Prosperi, Infrared behavior of the running coupling constant and bound states in QCD, Phys. Rev. D 66, 074008 (2002) [hep-ph/0202172].

[218] J. L. Richardson, The heavy quark potential and the Upsilon, J/psi Systems, Phys. Lett. B 82, 272 (1979).

[219] W. Buchmuller, G. Grunberg and S. H. H. Tye, The Regge Slope and the Lambda Parameter in QCD: An Empirical Approach via Quarkonia, Phys. Rev. Lett. 45, 103 (1980) [Phys. Rev. Lett. 45, 587 (1980)].

[220] Y. O. Belyakova and A. V. Nesterenko, A nonperturbative model for the strong running coupling within potential approach, Int. J. Mod. Phys. A 26, 981 (2011) [arXiv:1011.1148 [hep-ph]].

[221] C. Popovici, Dyson-Schwinger approach to strongly coupled theories, Mod. Phys. Lett. A 28, 1330006 (2013) [arXiv:1302.5642 [hep-ph]]; C. D. Roberts, Strong QCD and Dyson-Schwinger Equations, arXiv:1203.5341 [nucl-th]; A. Bashir, L. Chang, 
I. C. Cloet, B. El-Bennich, Y. X. Liu, C. D. Roberts and P. C. Tandy, Collective perspective on advances in Dyson-Schwinger Equation QCD, Commun. Theor. Phys. 58, 79 (2012) [arXiv:1201.3366 [nucl-th]]; C. D. Roberts and S. M. Schmidt, DysonSchwinger equations: Density, temperature and continuum strong QCD, Prog. Part. Nucl. Phys. 45, S1 (2000) [nucl-th/0005064]; C. D. Roberts and A. G. Williams, Dyson-Schwinger equations and their application to hadronic physics, Prog. Part. Nucl. Phys. 33, 477 (1994) [hep-ph/9403224].

[222] R. Alkofer and L. von Smekal, The Infrared behavior of QCD Green's functions: Confinement dynamical symmetry breaking, and hadrons as relativistic bound states, Phys. Rept. 353, 281 (2001) [hep-ph/0007355].

[223] C. S. Fischer, Infrared properties of QCD from Dyson-Schwinger equations, J. Phys. G 32, R253 (2006) [hep-ph/0605173].

[224] P. Boucaud, J. P. Leroy, A. L. Yaouanc, J. Micheli, O. Pene and J. RodriguezQuintero, The Infrared Behaviour of the Pure Yang-Mills Green Functions, Few Body Syst. 53, 387 (2012) [arXiv:1109.1936 [hep-ph]].

[225] A. Maas, Describing gauge bosons at zero and finite temperature, Phys. Rept. 524, 203 (2013) [arXiv:1106.3942 [hep-ph]].

[226] J. M. Cornwall, Dynamical Mass Generation in Continuum QCD, Phys. Rev. D 26, 1453 (1982).

[227] A. C. Aguilar, D. Binosi, J. Papavassiliou and J. Rodriguez-Quintero, Nonperturbative comparison of QCD effective charges, Phys. Rev. D 80, 085018 (2009) [arXiv:0906.2633 [hep-ph]].

[228] J. M. Cornwall, Positivity issues for the pinch-technique gluon propagator and their resolution, Phys. Rev. D 80, 096001 (2009) [arXiv:0904.3758 [hep-ph]].

[229] M. Baker, J. S. Ball and F. Zachariasen, A Nonperturbative Calculation of the Infrared Limit of the Axial Gauge Gluon Propagator. 1., Nucl. Phys. B 186, 531 (1981).

[230] J. C. R. Bloch, A. Cucchieri, K. Langfeld and T. Mendes, Propagators and running coupling from SU(2) lattice gauge theory, Nucl. Phys. B 687, 76 (2004) [heplat/0312036]; A. Cucchieri, T. Mendes and A. Mihara, Numerical study of the ghost-gluon vertex in Landau gauge, JHEP 0412, 012 (2004) [hep-lat/0408034]. 
[231] A. C. Aguilar and J. Papavassiliou, Infrared finite ghost propagator in the Feynman gauge, Phys. Rev. D 77, 125022 (2008) [arXiv:0712.0780 [hep-ph]].

[232] A. C. Aguilar and J. Papavassiliou, Power-law running of the effective gluon mass, Eur. Phys. J. A 35, 189 (2008) [arXiv:0708.4320 [hep-ph]].

[233] A. C. Aguilar and J. Papavassiliou, Gluon mass generation in the PT-BFM scheme, JHEP 0612, 012 (2006) [hep-ph/0610040].

[234] M. A. L. Capri, V. E. R. Lemes, R. F. Sobreiro, S. P. Sorella and R. Thibes, The Gluon and ghost propagators in Euclidean Yang-Mills theory in the maximal Abelian gauge: Taking into account the effects of the Gribov copies and of the dimension two condensates, Phys. Rev. D 77, 105023 (2008) [arXiv:0801.0566 [hepth]]; M. Q. Huber, K. Schwenzer and R. Alkofer, On the infrared scaling solution of SU(N) Yang-Mills theories in the maximally Abelian gauge, Eur. Phys. J. C 68, 581 (2010) [arXiv:0904.1873 [hep-th]].

[235] W. Schleifenbaum, M. Leder and H. Reinhardt, Infrared analysis of propagators and vertices of Yang-Mills theory in Landau and Coulomb gauge, Phys. Rev. D 73, 125019 (2006) [hep-th/0605115].

[236] A. C. Aguilar, D. Binosi and J. Papavassiliou, Yang-Mills two-point functions in linear covariant gauges, Phys. Rev. D 91, 085014 (2015) [arXiv:1501.07150 [hep$\mathrm{ph}]]$.

[237] D. Zwanziger, Nonperturbative Landau gauge and infrared critical exponents in QCD, Phys. Rev. D 65, 094039 (2002) [hep-th/0109224].

[238] L. von Smekal, R. Alkofer and A. Hauck, The Infrared behavior of gluon and ghost propagators in Landau gauge QCD, Phys. Rev. Lett. 79, 3591 (1997) [hepph/9705242]. L. von Smekal, A. Hauck and R. Alkofer, A Solution to Coupled Dyson-Schwinger Equations for Gluons and Ghosts in Landau Gauge, Annals Phys. 267, 1 (1998) [Annals Phys. 269, 182 (1998)] [hep-ph/9707327].

[239] D. Atkinson and J. C. R. Bloch, Running coupling in nonperturbative QCD. 1. Bare vertices and y-max approximation, Phys. Rev. D 58, 094036 (1998) [hepph/9712459]; D. Atkinson and J. C. R. Bloch, QCD in the infrared with exact angular integrations, Mod. Phys. Lett. A 13, 1055 (1998) [hep-ph/9802239]. 
[240] J. C. R. Bloch, Multiplicative renormalizability of gluon and ghost propagators in QCD, Phys. Rev. D 64, 116011 (2001) [hep-ph/0106031].

[241] C. Lerche and L. von Smekal, On the infrared exponent for gluon and ghost propagation in Landau gauge QCD, Phys. Rev. D 65, 125006 (2002) [hep-ph/0202194].

[242] C. S. Fischer, R. Alkofer and H. Reinhardt, The Elusiveness of infrared critical exponents in Landau gauge Yang-Mills theories, Phys. Rev. D 65, 094008 (2002) [hepph/0202195]; C. S. Fischer and R. Alkofer, Infrared exponents and running coupling of SU(N) Yang-Mills theories, Phys. Lett. B 536, 177 (2002) [hep-ph/0202202];

[243] R. Alkofer, C. S. Fischer and L. von Smekal, The Infrared behavior of the running coupling in Landau gauge QCD, Acta Phys. Slov. 52, 191 (2002) [hep-ph/0205125].

[244] R. Alkofer, W. Detmold, C. S. Fischer and P. Maris, Analytic properties of the Landau gauge gluon and quark propagators, Phys. Rev. D 70, 014014 (2004) [hep$\mathrm{ph} / 0309077]$.

[245] J. C. R. Bloch, Two loop improved truncation of the ghost gluon Dyson-Schwinger equations: Multiplicatively renormalizable propagators and nonperturbative running coupling, Few Body Syst. 33, 111 (2003) [hep-ph/0303125].

[246] C. S. Fischer and J. M. Pawlowski, Uniqueness of infrared asymptotics in Landau gauge Yang-Mills theory, Phys. Rev. D 75, 025012 (2007) [hep-th/0609009]. C. S. Fischer and J. M. Pawlowski, Uniqueness of infrared asymptotics in Landau gauge Yang-Mills theory II, Phys. Rev. D 80, 025023 (2009) [arXiv:0903.2193 [hep-th]].

[247] R. Alkofer, M. Q. Huber and K. Schwenzer, Infrared Behavior of Three-Point Functions in Landau Gauge Yang-Mills Theory, Eur. Phys. J. C 62, 761 (2009) [arXiv:0812.4045 [hep-ph]]; R. Alkofer, M. Q. Huber and K. Schwenzer, Infrared singularities in Landau gauge Yang-Mills theory, Phys. Rev. D 81, 105010 (2010) [arXiv:0801.2762 [hep-th]].

[248] M. Leder, J. M. Pawlowski, H. Reinhardt and A. Weber, Hamiltonian Flow in Coulomb Gauge Yang-Mills Theory, Phys. Rev. D 83, 025010 (2011) [arXiv:1006.5710 [hep-th]].

[249] C. S. Fischer and H. Gies, Renormalization flow of Yang-Mills propagators, JHEP 0410, 048 (2004) [hep-ph/0408089]. 
[250] J. M. Pawlowski, D. F. Litim, S. Nedelko and L. von Smekal, Infrared behavior and fixed points in Landau gauge QCD, Phys. Rev. Lett. 93, 152002 (2004) [hepth/0312324].

[251] S. Furui and H. Nakajima, What the Gribov copy tells about confinement and the theory of dynamical chiral symmetry breaking, Phys. Rev. D 70, 094504 (2004) [hep-lat/0403021]; S. Furui and H. Nakajima, Infrared features of unquenched Lattice Landau Gauge QCD, Few Body Syst. 40, 101 (2006) [hep-lat/0503029]; S. Furui, Propagator of the lattice domain wall fermion and the staggered fermion, Few Body Syst. 45, 51 (2009) [Few Body Syst. 46, 73 (2009)] [arXiv:0801.0325 [hep-lat]].

[252] R. Alkofer, C. S. Fischer, H. Reinhardt and L. von Smekal, On the infrared behavior of gluons and ghosts in ghost antighost symmetric gauges, Phys. Rev. D 68, 045003 (2003) [hep-th/0304134].

[253] G. Burgio, M. Quandt and H. Reinhardt, BRST symmetry versus Horizon condition in Yang-Mills theory, Phys. Rev. D 81, 074502 (2010) [arXiv:0911.5101 [heplat]].

[254] D. Epple, H. Reinhardt, W. Schleifenbaum and A. P. Szczepaniak, Subcritical solution of the Yang-Mills Schroedinger equation in the Coulomb gauge, Phys. Rev. D 77, 085007 (2008) [arXiv:0712.3694 [hep-th]].

[255] C. S. Fischer and M. R. Pennington, Finite volume effects in a quenched latticeQCD quark propagator, Phys. Rev. D 73, 034029 (2006) [hep-ph/0512233].

[256] M. Q. Huber, R. Alkofer, C. S. Fischer and K. Schwenzer, The Infrared behavior of Landau gauge Yang-Mills theory in $\mathrm{d}=2, \mathrm{~d}=3$ and $\mathrm{d}=4$ dimensions, Phys. Lett. B 659, 434 (2008) [arXiv:0705.3809 [hep-ph]]; M. Q. Huber, A. Maas and L. von Smekal, Two- and three-point functions in two-dimensional Landau-gauge YangMills theory: Continuum results, JHEP 1211, 035 (2012) [arXiv:1207.0222 [hepth]].

[257] P. Boucaud, J. P. Leroy, A. Le Yaouanc, A. Y. Lokhov, J. Micheli, O. Pene, J. Rodriguez-Quintero and C. Roiesnel, The Infrared behaviour of the pure YangMills green functions, hep-ph/0507104; P. Boucaud, J. P. Leroy, A. L. Yaouanc, J. Micheli, O. Pene and J. Rodriguez-Quintero, IR finiteness of the ghost dressing function from numerical resolution of the ghost SD equation, JHEP 0806, 
012 (2008) [arXiv:0801.2721 [hep-ph]]; P. Boucaud, J. P. Leroy, A. Le Yaouanc, J. Micheli, O. Pene and J. Rodriguez-Quintero, On the IR behaviour of the Landaugauge ghost propagator, JHEP 0806, 099 (2008) [arXiv:0803.2161 [hep-ph]].

[258] J. M. Cornwall and G. Tiktopoulos, On-Shell Asymptotics of Nonabelian Gauge Theories, Phys. Rev. Lett. 35, 338 (1975); J. M. Cornwall and G. Tiktopoulos, Infrared Behavior of Nonabelian Gauge Theories, Phys. Rev. D 13, 3370 (1976); J. M. Cornwall and G. Tiktopoulos, Infrared Behavior of Nonabelian Gauge Theories. 2., Phys. Rev. D 15, 2937 (1977); J. M. Cornwall, Quark Confinement and Vortices in Massive Gauge Invariant QCD, Nucl. Phys. B 157, 392 (1979).

[259] A. C. Aguilar and A. A. Natale, A Dynamical gluon mass solution in a coupled system of the Schwinger-Dyson equations, JHEP 0408, 057 (2004) [hep-ph/0408254].

[260] A. C. Aguilar, D. Binosi and J. Papavassiliou, Gluon and ghost propagators in the Landau gauge: Deriving lattice results from Schwinger-Dyson equations, Phys. Rev. D 78, 025010 (2008) [arXiv:0802.1870 [hep-ph]].

[261] C. S. Fischer, A. Maas and J. M. Pawlowski, On the infrared behavior of Landau gauge Yang-Mills theory, Annals Phys. 324, 2408 (2009) [arXiv:0810.1987 [hep-ph]].

[262] A. C. Aguilar, D. Binosi and J. Papavassiliou, Gluon mass through ghost synergy, JHEP 1201, 050 (2012) [arXiv:1108.5989 [hep-ph]].

[263] D. Dudal, O. Oliveira and N. Vandersickel, Indirect lattice evidence for the Refined Gribov-Zwanziger formalism and the gluon condensate $\left\langle A^{2}\right\rangle$ in the Landau gauge, Phys. Rev. D 81, 074505 (2010) [arXiv:1002.2374 [hep-lat]].

$[264]$

[265] D. Dudal, J. A. Gracey, S. P. Sorella, N. Vandersickel and H. Verschelde, A Refinement of the Gribov-Zwanziger approach in the Landau gauge: Infrared propagators in harmony with the lattice results, Phys. Rev. D 78, 065047 (2008) [arXiv:0806.4348 [hep-th]]; D. Dudal, S. P. Sorella and N. Vandersickel, The dynamical origin of the refinement of the Gribov-Zwanziger theory, Phys. Rev. D 84, 065039 (2011) [arXiv:1105.3371 [hep-th]].

[266] F. J. Llanes-Estrada and R. Williams, Two infrared Yang-Mills solutions in stochastic quantization and in an effective action formalism, Phys. Rev. D 86, 065034 (2012) [arXiv:1207.5950 [hep-th]]. 
[267] C. S. Fischer, A. Maas, J. M. Pawlowski and L. von Smekal, Large volume behaviour of Yang-Mills propagators, Annals Phys. 322, 2916 (2007) [hep$\mathrm{ph} / 0701050]$.

[268] C. Becchi, A. Rouet and R. Stora, Renormalization of Gauge Theories, Annals Phys. 98, 287 (1976). I. V. Tyutin, Gauge Invariance in Field Theory and Statistical Physics in Operator Formalism, arXiv:0812.0580 [hep-th].

[269] T. Kugo and I. Ojima, Local Covariant Operator Formalism of Nonabelian Gauge Theories and Quark Confinement Problem, Prog. Theor. Phys. Suppl. 66, 1 (1979); T. Kugo, The Universal renormalization factors Z(1) / Z(3) and color confinement condition in nonAbelian gauge theory, hep-th/9511033.

[270] P. Boucaud, M. E. Gomez, J. P. Leroy, A. Le Yaouanc, J. Micheli, O. Pene and J. Rodriguez-Quintero, The low-momentum ghost dressing function and the gluon mass, Phys. Rev. D 82, 054007 (2010) [arXiv:1004.4135 [hep-ph]].

[271] D. J. Thelan and J. A. Gracey, Natural solution in the refined Gribov-Zwanziger theory, Phys. Rev. D 89, 107701 (2014)

[272] J. S. Ball and F. Zachariasen, Infrared Properties of the Glue Propagator in Nonabelian Gauge Theories, Nucl. Phys. B 143, 148 (1978); R. Anishetty, M. Baker, S. K. Kim, J. S. Ball and F. Zachariasen, Infrared Properties of the Coupling Constant in Nonabelian Gauge Theories, Phys. Lett. B 86, 52 (1979); M. Baker, J. S. Ball, P. Lucht and F. Zachariasen, Infrared Properties of the Coupling Constant in Nonabelian Gauge Theories. 2., Phys. Lett. B 89, 211 (1980).

[273] S. Mandelstam, Approximation Scheme for QCD, Phys. Rev. D 20, 3223 (1979).

[274] U. Bar-Gadda, Infrared Behavior of the Effective Coupling in Quantum Chromodynamics: A Nonperturbative Approach, Nucl. Phys. B 163, 312 (1980).

[275] G. Parisi and R. Petronzio, On Low-Energy Tests of QCD, Phys. Lett. B 94, 51 (1980).

[276] E. C. Poggio, E. Tomboulis and S.-H. H. Tye, Dynamical Symmetry Breaking in Nonabelian Field Theories, Phys. Rev. D 11, 2839 (1975).

[277] J. Franklin and D. B. Lichtenberg, Estimate of the Quark - Gluon Coupling Strength From Baryon Masses, Phys. Rev. D 25, 1997 (1982). 
[278] D. Binosi and J. Papavassiliou, Pinch Technique: Theory and Applications, Phys. Rept. 479, 1 (2009) [arXiv:0909.2536 [hep-ph]]; J. M. Cornwall, J. Papavassiliou and D. Binosi, The Pinch Technique and its Applications to Non-Abelian Gauge Theories (Cambridge Monographs on Particle Physics, Nuclear Physics and Cosmology), Cambridge University Press (2011).

[279] C. R. Ji, A. F. Sill and R. M. Lombard, Leading Order Perturbative QCD Calculation of Nucleon Dirac Form-factors, Phys. Rev. D 36, 165 (1987); C. R. Ji and F. Amiri, Perturbative QCD Analysis of the Pion Form-factor Using a Frozen Coupling Constant, Phys. Rev. D 42, 3764 (1990).

[280] S. J. Brodsky, C. R. Ji, A. Pang and D. G. Robertson, Optimal renormalization scale and scheme for exclusive processes, Phys. Rev. D 57, 245 (1998) [hep$\mathrm{ph} / 9705221]$.

[281] A. C. Aguilar, A. Mihara and A. A. Natale, Phenomenological tests for the freezing of the QCD running coupling constant, Int. J. Mod. Phys. A 19, 249 (2004); A. C. Aguilar, A. Mihara and A. A. Natale, Freezing of the QCD coupling constant and solutions of Schwinger-Dyson equations, Phys. Rev. D 65, 054011 (2002) [hep-ph/0109223].

[282] A. C. Aguilar, A. Mihara and A. A. Natale, Testing the infrared behavior of the QCD running coupling constant, hep-ph/0208095.

[283] A. C. Aguilar, D. Binosi and J. Papavassiliou, The dynamical equation of the effective gluon mass, Phys. Rev. D 84, 085026 (2011) [arXiv:1107.3968 [hep-ph]]; A. C. Aguilar, D. Binosi and J. Papavassiliou, Unquenching the gluon propagator with Schwinger-Dyson equations, Phys. Rev. D 86, 014032 (2012) [arXiv:1204.3868 [hep-ph]]; A. C. Aguilar, D. Binosi and J. Papavassiliou, Gluon mass generation in the presence of dynamical quarks, Phys. Rev. D 88, 074010 (2013) [arXiv:1304.5936 [hep-ph]]; D. Binosi, D. Ibanez and J. Papavassiliou, The all-order equation of the effective gluon mass, Phys. Rev. D 86, 085033 (2012) [arXiv:1208.1451 [hep-ph]].

[284] A. C. Aguilar, D. Binosi and J. Papavassiliou, Renormalization group analysis of the gluon mass equation, Phys. Rev. D 89, 085032 (2014) [arXiv:1401.3631 [hep$\mathrm{ph}]]$.

[285] S. x. Qin, L. Chang, Y. x. Liu, C. D. Roberts and D. J. Wilson, Interaction model for the gap equation, Phys. Rev. C 84, 042202 (2011) [arXiv:1108.0603 
[nucl-th]]; S. x. Qin, L. Chang, Y. x. Liu, C. D. Roberts and D. J. Wilson, Investigation of rainbow-ladder truncation for excited and exotic mesons, Phys. Rev. C 85, 035202 (2012) [arXiv:1109.3459 [nucl-th]]; D. Binosi, L. Chang, J. Papavassiliou and C. D. Roberts, Bridging a gap between continuum-QCD and ab initio predictions of hadron observables, Phys. Lett. B 742, 183 (2015) [arXiv:1412.4782 [nucl-th]].

[286] C. W. Bernard, Monte Carlo Evaluation of the Effective Gluon Mass, Phys. Lett. B 108, 431 (1982); C. W. Bernard, Adjoint Wilson Lines and the Effective Gluon Mass, Nucl. Phys. B 219, 341 (1983); J. M. Cornwall and A. Soni, Glueballs as Bound States of Massive Gluons, Phys. Lett. B 120, 431 (1983); J. E. Mandula and M. Ogilvie, The Gluon Is Massive: A Lattice Calculation of the Gluon Propagator in the Landau Gauge, Phys. Lett. B 185, 127 (1987); F. Halzen, G. I. Krein and A. A. Natale, Relating the QCD pomeron to an effective gluon mass, Phys. Rev. D 47, 295 (1993).

[287] J. H. Field, A Phenomenological analysis of gluon mass effects in inclusive radiative decays of the J / psi and Upsilon, Phys. Rev. D 66, 013013 (2002) [hep-ph/0101158].

[288] C. Ayala, P. Gonzalez and V. Vento, Dynamical gluon mass and linear confinement, arXiv:1509.01382 [hep-ph].

[289] J. M. Cornwall and J. Papavassiliou, Gauge Invariant Three Gluon Vertex in QCD, Phys. Rev. D 40, 3474 (1989); J. Papavassiliou and J. M. Cornwall, Coupled fermion gap and vertex equations for chiral symmetry breakdown in QCD, Phys. Rev. D 44, 1285 (1991).

[290] M. Stingl, Propagation Properties and Condensate Formation of the Confined Yang-Mills Field, Phys. Rev. D 34, 3863 (1986) [Phys. Rev. D 36, 651 (1987)]; U. Habel, R. Konning, H. G. Reusch, M. Stingl and S. Wigard, A Nonperturbative Solution to the Dyson-schwinger Equations of Quantum Chromodynamics. 1. General Properties, Z. Phys. A 336, 423 (1990).

[291] D. B. Leinweber et al. [UKQCD Collaboration], Gluon propagator in the infrared region, Phys. Rev. D 58, 031501 (1998) [hep-lat/9803015]; D. B. Leinweber et al. [UKQCD Collaboration], Asymptotic scaling and infrared behavior of the gluon propagator, Phys. Rev. D 60, 094507 (1999) [Phys. Rev. D 61, 079901 (2000)] [hep-lat/9811027]. 
[292] P. Marenzoni, G. Martinelli and N. Stella, The Gluon propagator on a large volume, at beta $=6.0$, Nucl. Phys. B 455, 339 (1995) [hep-lat/9410011].

[293] O. Oliveira and P. Bicudo, Running Gluon Mass from Landau Gauge Lattice QCD Propagator, J. Phys. G 38, 045003 (2011) [arXiv:1002.4151 [hep-lat]].

[294] M. A. L. Capri, D. Dudal, V. E. R. Lemes, R. F. Sobreiro, S. P. Sorella, R. Thibes and H. Verschelde, The Gribov-Zwanziger action in the presence of the gauge invariant, nonlocal mass operator $\operatorname{Tr} \int d^{4} x F(\mu \nu)\left(D^{2}\right)^{-} 1 F(\mu \nu)$ in the Landau gauge, Eur. Phys. J. C 52, 459 (2007) [arXiv:0705.3591 [hep-th]].

[295] K. G. Chetyrkin, S. Narison and V. I. Zakharov, Short distance tachyonic gluon mass and $1 / Q^{* *} 2$ corrections, Nucl. Phys. B 550, 353 (1999) [hep-ph/9811275].

[296] J. C. R. Bloch, Multiplicative renormalizability and quark propagator, Phys. Rev. D 66, 034032 (2002) [hep-ph/0202073].

[297] M. Baker and C. k. Lee, Overlapping Divergence Free Skeleton Expansion in Nonabelian Gauge Theories, Phys. Rev. D 15, 2201 (1977) [Phys. Rev. D 17, 2182 (1978)].

[298] F. T. Brandt and J. Frenkel, Infrared Behavior of Three and Four Gluon Vertices in Yang-Mills Theory, Phys. Rev. D 33, 464 (1986).

[299] J. Papavassiliou, The Gauge invariant four gluon vertex and its ward identity, Phys. Rev. D 47, 4728 (1993).

[300] R. Alkofer, C. S. Fischer and F. J. Llanes-Estrada, Vertex functions and infrared fixed point in Landau gauge SU(N) Yang-Mills theory, Phys. Lett. B 611, 279 (2005) [Phys. Lett. 670, 460 (2009)]

[301] M. Pelaez, M. Tissier and N. Wschebor, Three-point correlation functions in YangMills theory, Phys. Rev. D 88, 125003 (2013) [arXiv:1310.2594 [hep-th]].

[302] G. Eichmann, R. Williams, R. Alkofer and M. Vujinovic, Three-gluon vertex in Landau gauge, Phys. Rev. D 89, 105014 (2014) [arXiv:1402.1365 [hep-ph]].

[303] A. Blum, M. Q. Huber, M. Mitter and L. von Smekal, Gluonic three-point correlations in pure Landau gauge QCD, Phys. Rev. D 89, 061703 (2014) [arXiv:1401.0713 [hep-ph]]. 
[304] M. Q. Huber, D. R. Campagnari and H. Reinhardt, Vertex functions of Coulomb gauge Yang-Mills theory, Phys. Rev. D 91, 025014 (2015) [arXiv:1410.4766 [hep$\mathrm{ph}]]$.

[305] M. Stingl, A Systematic extended iterative solution for quantum chromodynamics, Z. Phys. A 353, 423 (1996) [hep-th/9502157]; L. Driesen, J. Fromm, J. Kuhrs and M. Stingl, Extended iterative scheme for QCD: Three point vertices, Eur. Phys. J. A 4, 381 (1999) [hep-th/9808152]; L. Driesen and M. Stingl, Extended iterative scheme for QCD: The Four gluon vertex, Eur. Phys. J. A 4, 401 (1999) [hep-th/9808155].

[306] C. Kellermann and C. S. Fischer, The Running coupling from the four-gluon vertex in Landau gauge Yang-Mills theory, Phys. Rev. D 78, 025015 (2008) [arXiv:0801.2697 [hep-ph]].

[307] A. K. Cyrol, M. Q. Huber and L. von Smekal, A Dyson-Schwinger study of the four-gluon vertex, Eur. Phys. J. C 75, 102 (2015) [arXiv:1408.5409 [hep-ph]].

[308] C. S. Fischer and R. Alkofer, Nonperturbative propagators, running coupling and dynamical quark mass of Landau gauge QCD, Phys. Rev. D 67, 094020 (2003) [hep-ph/0301094]; C. S. Fischer, P. Watson and W. Cassing, Probing unquenching effects in the gluon polarisation in light mesons, Phys. Rev. D 72, 094025 (2005) [hep-ph/0509213]; C. S. Fischer, D. Nickel and J. Wambach, Hadronic unquenching effects in the quark propagator, Phys. Rev. D 76, 094009 (2007) [arXiv:0705.4407 [hep-ph]].

[309] R. Alkofer, C. S. Fischer and F. J. Llanes-Estrada, Dynamically induced scalar quark confinement, Mod. Phys. Lett. A 23, 1105 (2008) [hep-ph/0607293]; R. Alkofer, C. S. Fischer, F. J. Llanes-Estrada and K. Schwenzer, The Quark-gluon vertex in Landau gauge QCD: Its role in dynamical chiral symmetry breaking and quark confinement, Annals Phys. 324, 106 (2009) [arXiv:0804.3042 [hep-ph]].

[310] O. Philipsen and H. Wittig, String breaking in nonAbelian gauge theories with fundamental matter fields, Phys. Rev. Lett. 81, 4056 (1998) [Phys. Rev. Lett. 83, 2684 (1999)] [hep-lat/9807020]; F. Knechtli, String breaking and lines of constant physics in the SU(2) Higgs model, Nucl. Phys. Proc. Suppl. 83, 673 (2000) [heplat/9909164]; C. E. Detar, U. M. Heller and P. Lacock, First signs for string breaking in two flavor QCD, Nucl. Phys. Proc. Suppl. 83, 310 (2000) [hep-lat/9909078]; 
C. D. Roberts, Looking into the matter of light-quark hadrons, Few Body Syst. 52, 345 (2012) [arXiv:1109.6325 [nucl-th]].

[311] C. S. Fischer and D. Zwanziger, Infrared behaviour and running couplings in interpolating gauges in QCD, Phys. Rev. D 72, 054005 (2005) [hep-ph/0504244].

[312] R. Alkofer, A. Maas and D. Zwanziger, Truncating first-order Dyson-Schwinger equations in Coulomb-Gauge Yang-Mills theory, Few Body Syst. 47, 73 (2010) [arXiv:0905.4594 [hep-ph]].

[313] M. Q. Huber, Gluon and ghost propagators in linear covariant gauges, Phys. Rev. D 91, 085018 (2015) [arXiv:1502.04057 [hep-ph]].

[314] L. Giusti, M. L. Paciello, S. Petrarca, B. Taglienti and M. Testa, On the definition of gauge field operators in lattice gauge fixed theories, Phys. Lett. B 432, 196 (1998) [hep-lat/9803021]; L. Giusti, M. L. Paciello, C. Parrinello, S. Petrarca and B. Taglienti, Problems on lattice gauge fixing, Int. J. Mod. Phys. A 16, 3487 (2001) [hep-lat/0104012]; I. L. Bogolubsky and V. K. Mitrjushkin, Gluon propagators and the choice of the gauge field in SU(2) theory on the lattice, hep-lat/0204006.

[315] C. Parrinello, Exploratory study of the three gluon vertex on the lattice, Phys. Rev. D 50, 4247 (1994) [hep-lat/9405024].

[316] J. C. R. Bloch, A. Cucchieri, K. Langfeld and T. Mendes, Running coupling constant and propagators in SU(2) Landau gauge, Nucl. Phys. Proc. Suppl. 119, 736 (2003) [hep-lat/0209040]; K. Langfeld, J. C. R. Bloch, J. Gattnar, H. Reinhardt, A. Cucchieri and T. Mendes, Vortex induced confinement and the IR properties of Green functions, hep-th/0209173.

[317] J. Skullerud and A. Kizilersu, Quark gluon vertex from lattice QCD, JHEP 0209, 013 (2002) [hep-ph/0205318].

[318] O. Oliveira and P. J. Silva, Infrared Gluon and Ghost Propagators from Lattice QCD. Results from large asymmetric lattices, Eur. Phys. J. A 31, 790 (2007) [heplat/0609027]; O. Oliveira and P. J. Silva, Does The Lattice Zero Momentum Gluon Propagator for Pure Gauge SU(3) Yang-Mills Theory Vanish in the Infinite Volume Limit?, Phys. Rev. D 79, 031501 (2009) [arXiv:0809.0258 [hep-lat]].

[319] V. G. Bornyakov, V. K. Mitrjushkin and M. Muller-Preussker, Infrared behavior and Gribov ambiguity in $\mathrm{SU}(2)$ lattice gauge theory, Phys. Rev. D 79, 074504 
(2009) [arXiv:0812.2761 [hep-lat]]; V. G. Bornyakov, E. M. Ilgenfritz, C. Litwinski, V. K. Mitrjushkin and M. Muller-Preussker, Landau gauge ghost propagator and running coupling in SU(2) lattice gauge theory, Phys. Rev. D 92, 074505 (2015) [arXiv:1302.5943 [hep-lat]].

[320] E.-M. Ilgenfritz, M. Muller-Preussker, A. Sternbeck, A. Schiller and I. L. Bogolubsky, Landau gauge gluon and ghost propagators from lattice QCD, Braz. J. Phys. 37, 193 (2007) [hep-lat/0609043]; E. M. Ilgenfritz, C. Menz, M. Muller-Preussker, A. Schiller and A. Sternbeck, SU(3) Landau gauge gluon and ghost propagators using the logarithmic lattice gluon field definition, Phys. Rev. D 83, 054506 (2011) [arXiv:1010.5120 [hep-lat]].

[321] A. Sternbeck, E.-M. Ilgenfritz, M. Muller-Preussker and A. Schiller, Towards the infrared limit in SU(3) Landau gauge lattice gluodynamics, Phys. Rev. D 72, 014507 (2005) [hep-lat/0506007]; A. Sternbeck and M. Mller-Preussker, Lattice evidence for the family of decoupling solutions of Landau gauge Yang-Mills theory, Phys. Lett. B 726, 396 (2013) [arXiv:1211.3057 [hep-lat]].

[322] A. Cucchieri and T. Mendes, Landau-gauge propagators in Yang-Mills theories at beta $=0$ : Massive solution versus conformal scaling, Phys. Rev. D 81, 016005 (2010) [arXiv:0904.4033 [hep-lat]]; I. L. Bogolubsky, E. M. Ilgenfritz, M. Muller-Preussker and A. Sternbeck, Lattice gluodynamics computation of Landau gauge Green's functions in the deep infrared, Phys. Lett. B 676, 69 (2009) [arXiv:0901.0736 [hep-lat]]; O. Oliveira and P. J. Silva, The Lattice infrared Landau gauge gluon propagator: The Infinite volume limit, [arXiv:0910.2897 [hep-lat]]; O. Oliveira and P. J. Silva, The lattice infrared Landau gauge gluon propagator: from finite volume to the infinite volume, PoS QCD -TNT09, 033 (2009) [arXiv:0911.1643 [hep-lat]]; O. Oliveira and P. J. Silva, The lattice Landau gauge gluon propagator: lattice spacing and volume dependence, Phys. Rev. D 86, 114513 (2012) [arXiv:1207.3029 [hep-lat]].

[323] A. C. Aguilar, D. Binosi and J. Papavassiliou, QCD effective charges from lattice data, JHEP 1007, 002 (2010) [arXiv:1004.1105 [hep-ph]].

[324] S. Furui and H. Nakajima, Effects of the quark field on the ghost propagator of lattice Landau gauge QCD, Phys. Rev. D 73, 094506 (2006) [hep-lat/0602027]. 
[325] A. Ayala, A. Bashir, D. Binosi, M. Cristoforetti and J. Rodriguez-Quintero, Quark flavour effects on gluon and ghost propagators, Phys. Rev. D 86, 074512 (2012) [arXiv:1208.0795 [hep-ph]].

[326] A. Maas, Some more details of minimal-Landau-gauge SU(2) Yang-Mills propagators, Phys. Rev. D 91, 034502 (2015) [arXiv:1402.5050 [hep-lat]].

[327] A. Maas, A. Cucchieri and T. Mendes, On the infrared behavior of Green's functions in Yang-Mills theory, [hep-lat/0610006].

[328] A. Cucchieri and T. Mendes, Constraints on the IR behavior of the gluon propagator in Yang-Mills theories, Phys. Rev. Lett. 100, 241601 (2008) [arXiv:0712.3517 [hep-lat]]. A. Cucchieri and T. Mendes, Constraints on the IR behavior of the ghost propagator in Yang-Mills theories, Phys. Rev. D 78, 094503 (2008) [arXiv:0804.2371 [hep-lat]].

[329] C. Alexandrou, P. de Forcrand and E. Follana, The Gluon propagator without lattice Gribov copies on a finer lattice, Phys. Rev. D 65, 114508 (2002) [heplat/0112043]; C. Alexandrou, P. De Forcrand and E. Follana, The Laplacian gauge gluon propagator in SU(N(c)), Phys. Rev. D 65, 117502 (2002) [hep-lat/0203006].

[330] P. O. Bowman, U. M. Heller, D. B. Leinweber, M. B. Parappilly and A. G. Williams, Unquenched gluon propagator in Landau gauge, Phys. Rev. D 70, 034509 (2004) [hep-lat/0402032]; P. O. Bowman, U. M. Heller, D. B. Leinweber, M. B. Parappilly, A. G. Williams and J. b. Zhang, Unquenched quark propagator in Landau gauge, Phys. Rev. D 71, 054507 (2005) [hep-lat/0501019]; P. O. Bowman, U. M. Heller, D. B. Leinweber, M. B. Parappilly, A. Sternbeck, L. von Smekal, A. G. Williams and J. b. Zhang, Scaling behavior and positivity violation of the gluon propagator in full QCD, Phys. Rev. D 76, 094505 (2007) [hep-lat/0703022 [HEP-LAT]]; W. Kamleh, P. O. Bowman, D. B. Leinweber, A. G. Williams and J. Zhang, Unquenching effects in the quark and gluon propagator, Phys. Rev. D 76, 094501 (2007) [arXiv:0705.4129 [hep-lat]].

[331] F. D. R. Bonnet, P. O. Bowman, D. B. Leinweber and A. G. Williams, Infrared behavior of the gluon propagator on a large volume lattice, Phys. Rev. D 62, 051501 (2000) [hep-lat/0002020]; F. D. R. Bonnet, P. O. Bowman, D. B. Leinweber, A. G. Williams and J. M. Zanotti, Infinite volume and continuum limits of the Landau gauge gluon propagator, Phys. Rev. D 64, 034501 (2001) [hep-lat/0101013]. 
[332] R. Horsley, H. Perlt, P. E. L. Rakow, G. Schierholz and A. Schiller, The SU(3) Beta Function from Numerical Stochastic Perturbation Theory, Phys. Lett. B 728, 1 (2014) [arXiv:1309.4311 [hep-lat]].

[333] N. Vandersickel and D. Zwanziger, The Gribov problem and QCD dynamics, Phys. Rept. 520, 175 (2012) [arXiv:1202.1491 [hep-th]].

[334] F. Shoji, T. Suzuki, H. Kodama and A. Nakamura, A New gauge fixing method for Abelian projection, Phys. Lett. B 476, 199 (2000) [hep-lat/9910042]; H. Aiso et al. [Nal-Yamagata Collaboration], Gauge fixing and gluon propagators, Prog. Theor. Phys. Suppl. 122, 123 (1996); H. Aiso, J. Fromm, M. Fukuda, T. Iwamiya, A. Nakamura, M. Stingl and M. Yoshida, Towards understanding of confinement of gluons, Nucl. Phys. Proc. Suppl. 53, 570 (1997). A. Nakamura, T. Saito and S. Sakai, Lattice calculation of gluon screening masses, Phys. Rev. D 69, 014506 (2004) [hep-lat/0311024].

[335] M. Luscher, R. Sommer, P. Weisz and U. Wolff, A Precise determination of the running coupling in the SU(3) Yang-Mills theory, Nucl. Phys. B 413, 481 (1994) [hep-lat/9309005]; M. Luscher, R. Sommer, U. Wolff and P. Weisz, Computation of the running coupling in the SU(2) Yang-Mills theory, Nucl. Phys. B 389, 247 (1993) [hep-lat/9207010].

[336] C. Wetterich, Exact evolution equation for the effective potential, Phys. Lett. B 301, 90 (1993). T. R. Morris, The Exact renormalization group and approximate solutions, Int. J. Mod. Phys. A 9, 2411 (1994) [hep-ph/9308265].

[337] H. Gies, Running coupling in Yang-Mills theory: A flow equation study, Phys. Rev. D 66, 025006 (2002) [hep-th/0202207].

[338] J. Berges, N. Tetradis and C. Wetterich, Nonperturbative renormalization flow in quantum field theory and statistical physics, Phys. Rept. 363, 223 (2002) [hep$\mathrm{ph} / 0005122]$. H. Gies, Introduction to the functional RG and applications to gauge theories, Lect. Notes Phys. 852, 287 (2012) [hep-ph/0611146].

[339] U. Ellwanger, M. Hirsch and A. Weber, Flow equations for the relevant part of the pure Yang-Mills action, Z. Phys. C 69, 687 (1996) [hep-th/9506019]. B. Bergerhoff and C. Wetterich, Effective quark interactions and QCD propagators, Phys. Rev. D 57, 1591 (1998) [hep-ph/9708425]. 
[340] M. Semenov-Tyan-Shanskii and V. Franke, Zap. Nauch. Sem. Leningrad. Otdeleniya Matematicheskogo Instituta im V. A. Steklov, AN SSSR, vol 120, (1982) 159 (New York: Plenum Press 1986); R. Friedberg, T. D. Lee, Y. Pang and H. C. Ren, A Soluble gauge model with Gribov type copies, Annals Phys. 246, 381 (1996). G. Dell'Antonio and D. Zwanziger, All gauge orbits and some Gribov copies encompassed by the Gribov horizon, Proceedings of the NATO Advanced Workshop on Probabilistic Methods in Quantum Field Theory and Quantum Gravity, Cargese 1989, Plenum. P. Damgaard, H. Huffel and A. Rosenblum, Eds.

[341] D. Zwanziger, Fundamental modular region, Boltzmann factor and area law in lattice gauge theory, Nucl. Phys. B 412, 657 (1994).

[342] D. Zwanziger, Nonperturbative Faddeev-Popov formula and infrared limit of QCD, Phys. Rev. D 69, 016002 (2004) [hep-ph/0303028].

[343] A. Cucchieri, Gribov copies in the minimal Landau gauge: The Influence on gluon and ghost propagators, Nucl. Phys. B 508, 353 (1997) [hep-lat/9705005]; J. E. Mandula, The gluon propagator, Phys. Rept. 315, 273 (1999) [hep-lat/9907020]; A. Cucchieri and D. Zwanziger, Numerical study of gluon propagator and confinement scenario in minimal Coulomb gauge, Phys. Rev. D 65, 014001 (2001) [hep-lat/0008026]; P. O. Bowman, U. M. Heller, D. B. Leinweber and A. G. Williams, Gluon propagator on coarse lattices in Laplacian gauges, Phys. Rev. D 66, 074505 (2002) [heplat/0206010]; A. Cucchieri, T. Mendes and A. R. Taurines, SU(2) Landau gluon propagator on a 140**3 lattice, Phys. Rev. D 67, 091502 (2003) [hep-lat/0302022]; J. B. Zhang, P. O. Bowman, R. J. Coad, U. M. Heller, D. B. Leinweber and A. G. Williams, Quark propagator in Landau and Laplacian gauges with overlap fermions, Phys. Rev. D 71, 014501 (2005) [hep-lat/0410045].

[344] D. Dudal, R. F. Sobreiro, S. P. Sorella and H. Verschelde, The Gribov parameter and the dimension two gluon condensate in Euclidean Yang-Mills theories in the Landau gauge, Phys. Rev. D 72, 014016 (2005) [hep-th/0502183]; D. Dudal, S. P. Sorella, N. Vandersickel and H. Verschelde, New features of the gluon and ghost propagator in the infrared region from the Gribov-Zwanziger approach, Phys. Rev. D 77, 071501 (2008) [arXiv:0711.4496 [hep-th]].

[345] J. A. Gracey, One loop gluon form factor and freezing of alpha(s) in the GribovZwanziger QCD Lagrangian, JHEP 0605, 052 (2006) [JHEP 1002, 078 (2010)] [hep-ph/0605077]. 
[346] J. A. Gracey, Low momentum propagators at two loops in gluon mass model, J. Phys. A 47, 445401 (2014) [arXiv:1409.0455 [hep-ph]].

[347] A. G. Williams, Nonperturbative QCD, gauge fixing, Gribov copies, and the lattice, Prog. Theor. Phys. Suppl. 151, 154 (2003) [hep-lat/0304003].

[348] E. Nelson, Derivation of the Schrodinger equation from Newtonian mechanics, Phys. Rev. 150, 1079 (1966); G. Parisi and Y. s. Wu, Perturbation Theory Without Gauge Fixing, Sci. Sin. 24, 483 (1981).

[349] D. Zwanziger, Time independent stochastic quantization, DS equations, and infrared critical exponents in QCD, Phys. Rev. D 67, 105001 (2003) [hep-th/0206053].

[350] A. Munoz Sudupe and R. F. Alvarez-Estrada, Renormalization Constants For The Propagator Of The Stochastically Quantized Yang-mills Field Theory, Phys. Lett. B 164, 102 (1985); A. Munoz Sudupe and R. F. Alvarez-Estrada, Beta Function for Yang-Mills Field Theory in Stochastic Quantization, Phys. Lett. B 166, 186 (1986); A. Munoz Sudupe, Renormalization Group Analysis Of Yang-mills Field Theory Stochastically Quantized, Phys. Lett. B 167, 221 (1986); L. Sadun, Continuum Regularization Of Quantum Field Theory. 5. Schwinger-dyson Renormalization, Z. Phys. C 36, 407 (1987); K. Okano, Background Field Method in Stochastic Quantization, Nucl. Phys. B 289, 109 (1987); K. Okano and L. Schulke, Renormalization In Stochastic Quantization And Supersymmetry, Prog. Theor. Phys. Suppl. 111, 203 (1993).

[351] J. M. Pawlowski, D. Spielmann and I. O. Stamatescu, Lattice Landau gauge with stochastic quantisation, Nucl. Phys. B 830, 291 (2010) [arXiv:0911.4921 [hep-lat]].

[352] P. J. Redmond and J. L. Uretsky, Conjecture concerning the properties of nonrenormalizable field theories, Phys. Rev. Lett. 1, 147 (1958).

[353] D. V. Shirkov and I. L. Solovtsov, Analytic QCD running coupling with finite IR behavior and universal alpha-s (0) value, hep-ph/9604363; D. V. Shirkov and I. L. Solovtsov, Analytic model for the QCD running coupling with universal alpha-s (0) value, Phys. Rev. Lett. 79, 1209 (1997) [hep-ph/9704333]; A. I. Alekseev, Nonperturbative power corrections to two-loop $\alpha_{s}\left(Q^{2}\right)$ in an analytic approach to QCD, Phys. Rev. D 61, 114005 (2000); A. I. Alekseev, Nonperturbative contributions in an analytic running coupling of QCD, J. Phys. G 27, L117 (2001) [hep-ph/0105338]; 
A. I. Alekseev, Effective method for calculating the analytic QCD coupling constant, Phys. Atom. Nucl. 65, 1678 (2002) [Yad. Fiz. 65, 1722 (2002)].

[354] A. I. Alekseev, Strong coupling constant to four loops in the analytic approach to QCD, Few Body Syst. 32, 193 (2003) [hep-ph/0211339].

[355] D. V. Shirkov and I. L. Solovtsov, Ten years of the Analytic Perturbation Theory in QCD, Theor. Math. Phys. 150, 132 (2007) [hep-ph/0611229]; D. Shirkov, Large regular QCD coupling at Low Energy?, arXiv:0807.1404 [hep-ph]; N. G. Stefanis, Taming Landau singularities in QCD perturbation theory: The Analytic approach, Phys. Part. Nucl. 44, 494 (2013) [arXiv:0902.4805 [hep-ph]].

[356] D. V. Shirkov, The Unitary mechanism of infrared freezing in QCD with massive gluons, Phys. Atom. Nucl. 62, 1928 (1999) [Yad. Fiz. 62, 2082 (1999)] [hep$\mathrm{ph} / 9903431]$.

[357] A. V. Kotikov, V. G. Krivokhizhin and B. G. Shaikhatdenov, Analytic and 'frozen' QCD coupling constants up to NNLO from DIS data, Phys. Atom. Nucl. 75, 507 (2012) [arXiv:1008.0545 [hep-ph]].

[358] D. V. Shirkov, Renorm - group, causality and nonpower perturbation expansion in QFT, Theor. Math. Phys. 119, 438 (1999) [Teor. Mat. Fiz. 119, 55 (1999)] [hep-th/9810246]; I. L. Solovtsov and D. V. Shirkov, Analytic approach in quantum chromodynamics, Theor. Math. Phys. 120, 1220 (1999) [Teor. Mat. Fiz. 120, 482 (1999)] [hep-ph/9909305]; A. I. Karanikas and N. G. Stefanis, Analyticity and power corrections in hard scattering hadronic functions, Phys. Lett. B 504, 225 (2001) [Phys. Lett. B 636, 330 (2006)] [hep-ph/0101031].

[359] K. A. Milton, I. L. Solovtsov and O. P. Solovtsova, Analytic perturbation theory and inclusive tau decay, Phys. Lett. B 415, 104 (1997) [hep-ph/9706409].

[360] A. P. Bakulev, K. Passek-Kumericki, W. Schroers and N. G. Stefanis, Pion formfactor in QCD: From nonlocal condensates to NLO analytic perturbation theory, Phys. Rev. D 70, 033014 (2004) [Phys. Rev. D 70, 079906 (2004)] [hep-ph/0405062].

[361] D. S. Kurashev and B. A. Magradze, Explicit expressions for timelike and spacelike observables of quantum chromodynamics in analytic perturbation theory, Theor. Math. Phys. 135, 531 (2003) [Teor. Mat. Fiz. 135, 95 (2003)]; B. A. Magradze, Practical techniques of analytic perturbation theory of QCD, hep-ph/0305020. 
[362] A. V. Nesterenko, Quark - anti-quark potential in the analytic approach to QCD, Phys. Rev. D 62, 094028 (2000) [hep-ph/9912351]; A. V. Nesterenko, Nonperturbative aspects of the QCD analytic invariant charge, Nucl. Phys. Proc. Suppl. 133, 59 (2004) [hep-ph/0307283].

[363] N. G. Stefanis, W. Schroers and H. C. Kim, Analytic coupling and Sudakov effects in exclusive processes: Pion and gamma* gamma - $i$ pi0 form-factors, Eur. Phys. J. C 18, 137 (2000) [hep-ph/0005218].

[364] D. V. Shirkov, Analytic perturbation theory in analyzing some QCD observables, Eur. Phys. J. C 22, 331 (2001) [hep-ph/0107282].

[365] K. A. Milton, I. L. Solovtsov, O. P. Solovtsova and V. I. Yasnov, Renormalization scheme and higher loop stability in hadronic tau decay within analytic perturbation theory, Eur. Phys. J. C 14, 495 (2000) [hep-ph/0003030]; B. V. Geshkenbein, B. L. Ioffe and K. N. Zyablyuk, The Check of QCD based on the tau - decay data analysis in the complex q**2 - plane, Phys. Rev. D 64, 093009 (2001) [hep$\mathrm{ph} / 0104048]$.

[366] G. Cvetic, R. Kogerler and C. Valenzuela, Analytic QCD coupling with no power terms in UV regime, J. Phys. G 37, 075001 (2010) [arXiv:0912.2466 [hep-ph]]; G. Cvetic, R. Kogerler and C. Valenzuela, Reconciling the analytic QCD with the ITEP operator product expansion philosophy, Phys. Rev. D 82, 114004 (2010) [arXiv:1006.4199 [hep-ph]].

[367] M. Baldicchi and G. M. Prosperi, Regge trajectories and quarkonium spectrum from a first principle Salpeter equation, Phys. Lett. B 436, 145 (1998) [hepph/9803390]; G. M. Prosperi and M. Baldicchi, BS and DS equations in a Wilson loop context in QCD, effective mass operator, q anti-q spectrum, Fizika B 8, 251 (1999) [hep-ph/9902346]; M. Baldicchi and G. M. Prosperi, $B_{c}$ meson and the light - heavy quarkonium spectrum, Phys. Rev. D 62, 114024 (2000) [hep-ph/0008017]; M. Baldicchi and G. M. Prosperi, Running coupling constant and masses in QCD, the meson spectrum, AIP Conf. Proc. 756, 152 (2005) [hep-ph/0412359].

[368] G. Ganbold, QCD Effective Coupling in the Infrared Region, Phys. Rev. D 81, 094008 (2010) [arXiv:1004.5280 [hep-ph]].

[369] K. A. Milton, I. L. Solovtsov and O. P. Solovtsova, The Bjorken sum rule in the analytic approach to perturbative QCD, Phys. Lett. B 439, 421 (1998) [hep- 
ph/9809510]; R. S. Pasechnik, D. V. Shirkov and O. V. Teryaev, Bjorken Sum Rule and pQCD frontier on the move, Phys. Rev. D 78, 071902 (2008) [arXiv:0808.0066 [hep-ph]]; R. S. Pasechnik, D. V. Shirkov, O. V. Teryaev, O. P. Solovtsova and V. L. Khandramai, Nucleon spin structure and pQCD frontier on the move, Phys. Rev. D 81, 016010 (2010) [arXiv:0911.3297 [hep-ph]]; R. S. Pasechnik, J. Soffer and O. V. Teryaev, Nucleon spin structure at low momentum transfers, Phys. Rev. D 82, 076007 (2010) [arXiv:1009.3355 [hep-ph]]; V. L. Khandramai, R. S. Pasechnik, D. V. Shirkov, O. P. Solovtsova and O. V. Teryaev, Four-loop QCD analysis of the Bjorken sum rule vs data, Phys. Lett. B 706, 340 (2012) [arXiv:1106.6352 [hep-ph]].

[370] A. P. Bakulev, S. V. Mikhailov and N. G. Stefanis, QCD analytic perturbation theory: From integer powers to any power of the running coupling, Phys. Rev. D 72, 074014 (2005) [Phys. Rev. D 72, 119908 (2005)] [hep-ph/0506311]; A. P. Bakulev, S. V. Mikhailov and N. G. Stefanis, Fractional Analytic Perturbation Theory in Minkowski space and application to Higgs boson decay into a b anti-b pair, Phys. Rev. D 75, 056005 (2007) [Phys. Rev. D 77, 079901 (2008)] [hep-ph/0607040]; A. P. Bakulev, S. V. Mikhailov and N. G. Stefanis, Higher-order QCD perturbation theory in different schemes: From FOPT to CIPT to FAPT, JHEP 1006, 085 (2010) [arXiv:1004.4125 [hep-ph]].

[371] A. V. Nesterenko, New analytic running coupling in space - like and time - like regions, Phys. Rev. D 64, 116009 (2001) [hep-ph/0102124]; A. V. Nesterenko and I. L. Solovtsov, New analytic running coupling in QCD: Higher loop levels, Mod. Phys. Lett. A 16, 2517 (2001) [hep-ph/0111067]; A. V. Nesterenko, Analytic invariant charge in QCD, Int. J. Mod. Phys. A 18, 5475 (2003) [hep-ph/0308288]; A. V. Nesterenko, Analytic invariant charge and the lattice static quark anti-quark potential, Int. J. Mod. Phys. A 19, 3471 (2004) [hep-ph/0305091].

[372] A. V. Nesterenko and J. Papavassiliou, The Massive analytic invariant charge in QCD, Phys. Rev. D 71, 016009 (2005) [hep-ph/0410406].

[373] F. Schrempp, Tracking QCD instantons, J. Phys. G 28, 915 (2002) [hepph/0109032]; D. Klammer and F. Schrempp, QCD-Instantons and Conformal Space-Time Inversion Symmetry, JHEP 0806, 098 (2008) [arXiv:0804.4573 [hep$\mathrm{ph}]]$. 
[374] A. C. Aguilar, A. V. Nesterenko and J. Papavassiliou, Infrared enhanced analytic coupling and chiral symmetry breaking in QCD, J. Phys. G 31, 997 (2005) [hep$\mathrm{ph} / 0504195]$.

[375] A. I. Alekseev and B. A. Arbuzov, An Invariant charge model for all $q^{2}>0$ in QCD and gluon condensate, Mod. Phys. Lett. A 20, 103 (2005) [hep-ph/0411339]; A. I. Alekseev and B. A. Arbuzov, Analyticity and minimality of nonperturbative contributions in perturbative region for alpha-s, Mod. Phys. Lett. A 13, 1747 (1998) [hep-ph/9704228]; A. I. Alekseev, Analytic invariant charge in QCD with suppression of nonperturbative contributions at large $\mathrm{Q}^{* *}$, Theor. Math. Phys. 145, 1559 (2005) [Teor. Mat. Fiz. 145, 221 (2005)]; A. I. Alekseev, Synthetic running coupling of QCD, Few Body Syst. 40, 57 (2006) [hep-ph/0503242].

[376] D. V. Shirkov, 'Massive' Perturbative QCD, regular in the IR limit, Phys. Part. Nucl. Lett. 10, 186 (2013) [arXiv:1208.2103 [hep-th]].

[377] C. Ayala and G. Cvetic, Mathematica and Fortran programs for various analytic QCD couplings, J. Phys. Conf. Ser. 608, 012064 (2015) [arXiv:1411.1581 [hep-ph]].

[378] L. F. Abbott, The Background Field Method Beyond One Loop, Nucl. Phys. B 185, 189 (1981).

[379] A. M. Badalian and Y. A. Simonov, Freezing of alpha $(\mathrm{s})\left(\mathrm{Q}^{* *} 2\right)$ in e+ e- annihilation, Phys. Atom. Nucl. 60, 630 (1997) [Yad. Fiz. 60, 714 (1997)].

[380] Y. A. Simonov, Asymptotic freedom and IR freezing in QCD: the role of gluon paramagnetism, Phys. Atom. Nucl. 74, 1223 (2011) [arXiv:1011.5386 [hep-ph]].

[381] A. M. Badalian and D. S. Kuzmenko, Freezing of QCD coupling alpha(s) affects the short distance static potential, Phys. Rev. D 65, 016004 (2001) [hep-ph/0104097].

[382] A. C. Mattingly and P. M. Stevenson, QCD perturbation theory at lowenergies, Phys. Rev. Lett. 69, 1320 (1992) [hep-ph/9207228]; A. C. Mattingly and P. M. Stevenson, Optimization of R(e+ e-) and 'freezing' of the QCD couplant at low-energies, Phys. Rev. D 49, 437 (1994) [hep-ph/9307266].

[383] A. Courtoy and S. Liuti, Extraction of $\alpha_{s}$ from deep inelastic scattering at large x, Phys. Lett. B 726, 320 (2013) [arXiv:1302.4439 [hep-ph]]. 
[384] M. Frasca, Infrared Gluon and Ghost Propagators, Phys. Lett. B 670, 73 (2008) [arXiv:0709.2042 [hep-th]]; M. Frasca, Yang-Mills Propagators and QCD, Nucl. Phys. Proc. Suppl. 186, 260 (2009) [arXiv:0807.4299 [hep-ph]]. M. Frasca, Mapping a Massless Scalar Field Theory on a Yang-Mills Theory: Classical Case, Mod. Phys. Lett. A 24, 2425 (2009) [arXiv:0903.2357 [math-ph]].

[385] N. N. Bogoliubov, Sov. Phys.-Usp. 67 (1959) 236; For a review, see B. A. Arbuzov, Non-perturbative Effective Interactions in the Standard Model. De Gruyter (2014).

[386] M. Tissier and N. Wschebor, Infrared propagators of Yang-Mills theory from perturbation theory, Phys. Rev. D 82, 101701 (2010) [arXiv:1004.1607 [hep-ph]]; M. Tissier and N. Wschebor, An Infrared Safe perturbative approach to Yang-Mills correlators, Phys. Rev. D 84, 045018 (2011) [arXiv:1105.2475 [hep-th]]; M. Pelaez, M. Tissier and N. Wschebor, Three-point correlation functions in Yang-Mills theory, Phys. Rev. D 88, 125003 (2013) [arXiv:1310.2594 [hep-th]]; M. Pelez, M. Tissier and N. Wschebor, Two-point correlation functions of QCD in the Landau gauge, Phys. Rev. D 90, 065031 (2014) [arXiv:1407.2005 [hep-th]]; M. Pelez, M. Tissier and N. Wschebor, Quark-gluon vertex from the Landau gauge Curci-Ferrari model, Phys. Rev. D 92, 045012 (2015) [arXiv:1504.05157 [hep-th]]; J. Serreau, M. Tissier and A. Tresmontant, Covariant gauges without Gribov ambiguities in Yang-Mills theories, Phys. Rev. D 89, 125019 (2014) [arXiv:1307.6019 [hep-th]]; J. Serreau and M. Tissier, Lifting the Gribov ambiguity in Yang-Mills theories, Phys. Lett. B 712, 97 (2012) [arXiv:1202.3432 [hep-th]].

[387] G. Curci and R. Ferrari, On a Class of Lagrangian Models for Massive and Massless Yang-Mills Fields, Nuovo Cim. A 32, 151 (1976).

[388] A. Deur, S. J. Brodsky and G. F. de Teramond, On the interface between perturbative and nonperturbative QCD, arXiv:1601.06568 [hep-ph] (To be published in Phys. Lett. B).

[389] T. A. Ryttov and R. Shrock, Comparison of Some Exact and Perturbative Results for a Supersymmetric $\mathrm{SU}\left(N_{C}\right)$ Gauge Theory, Phys. Rev. D 85, 076009 (2012) [arXiv:1202.1297 [hep-ph]]; T. A. Ryttov and R. Shrock, Scheme Transformations in the Vicinity of an Infrared Fixed Point, Phys. Rev. D 86, 065032 (2012) [arXiv:1206.2366 [hep-ph]]; T. A. Ryttov and R. Shrock, An Analysis of Scheme Transformations in the Vicinity of an Infrared Fixed Point, Phys. Rev. D 
86, 085005 (2012) [arXiv:1206.6895 [hep-th]]; R. Shrock, Study of Scheme Transformations to Remove Higher-Loop Terms in the $\beta$ Function of a Gauge Theory, Phys. Rev. D 88, 036003 (2013) [arXiv:1305.6524 [hep-ph]]; R. Shrock, Generalized Scheme Transformations for the Elimination of Higher-Loop Terms in the Beta Function of a Gauge Theory, Phys. Rev. D 90, 045011 (2014) [arXiv:1405.6244 [hep-th]]; G. Choi and R. Shrock, New Scheme Transformations and Application to Study Scheme Dependence of an Infrared Zero of the Beta Function in Gauge Theories, Phys. Rev. D 90, 125029 (2014) [arXiv:1411.6645 [hep-th]].

[390] G. Grunberg, Method of effective charges and BLM criterion, Phys. Rev. D 46, 2228 (1992).

[391] E. Gardi and M. Karliner, Relations between observables and the infrared fixed point in QCD, Nucl. Phys. B 529, 383 (1998) [hep-ph/9802218].

[392] T. A. Ryttov, Higher loop corrections to the infrared evolution of fermionic gauge theories in the RI' scheme, Phys. Rev. D 89, 016013 (2014) [arXiv:1309.3867 [hepph]]; T. A. Ryttov, Infrared fixed points in the minimal momentum subtraction scheme, Phys. Rev. D 89, 056001 (2014) [arXiv:1311.0848 [hep-ph]]; T. A. Ryttov, Conformal Behavior at Four Loops and Scheme (In)Dependence, Phys. Rev. D 90, 056007 (2014) [Phys. Rev. D 91, 039906 (2015)] [arXiv:1408.5841 [hep-th]].

[393] A. Maas, More on Gribov copies and propagators in Landau-gauge Yang-Mills theory, Phys. Rev. D 79, 014505 (2009) [arXiv:0808.3047 [hep-lat]].

[394] M. Q. Huber and L. von Smekal, On the influence of three-point functions on the propagators of Landau gauge Yang-Mills theory, JHEP 1304, 149 (2013) [arXiv:1211.6092 [hep-th]].

[395] U. Glassner et al. [TXL Collaboration], First evidence of N(f) dependence in the QCD interquark potential, Phys. Lett. B 383, 98 (1996) [hep-lat/9604014]; C. W. Bernard et al., The Static quark potential in three flavor QCD, Phys. Rev. D 62, 034503 (2000) [hep-lat/0002028].

[396] S. Titard and F. J. Yndurain, Rigorous QCD evaluation of spectrum and ground state properties of heavy q anti-q systems: With a precision determination of $\mathrm{m}(\mathrm{b})$ M(eta(b)), Phys. Rev. D 49, 6007 (1994) [hep-ph/9310236]; S. Titard and F. J. Yndurain, Rigorous QCD evaluation of spectrum and other properties of heavy $q$ 
anti-q systems. 2. Bottomium with $\mathrm{n}=2, \mathrm{l}=0$, 1, Phys. Rev. D 51, 6348 (1995) [hep-ph/9403400]; S. Titard and F. J. Yndurain, The $1=1$ hyperfine splitting in bottomonium as a precise probe of the QCD vacuum, Phys. Lett. B 351, 541 (1995) [hep-ph/9501338]; A. Pineda, More nonperturbative corrections to the fine and hyperfine splitting in the heavy quarkonium, Phys. Rev. D 55, 407 (1997) [Phys. Rev. D 59, 099901 (1999)] [hep-ph/9605424]; A. Pineda and F. J. Yndurain, Calculation of quarkonium spectrum and $\mathrm{m}(\mathrm{b}), \mathrm{m}(\mathrm{c})$ to order alpha-s**4, Phys. Rev. D 58, 094022 (1998) [hep-ph/9711287].

[397] J. D. Gomez and A. A. Natale, $R_{e^{+} e^{-}}$and an effective QCD charge, arXiv:1509.04798 [hep-ph].

[398] A. M. Badalian and V. L. Morgunov, Determination of alpha(s)(1-GeV) from the charmonium fine structure, Phys. Rev. D 60, 116008 (1999) [hep-ph/9901430]; A. M. Badalian and B. L. G. Bakker, Strong coupling constant from bottomonium fine structure, Phys. Rev. D 62, 094031 (2000) [hep-ph/0004021]. A. M. Badalian, A. I. Veselov and B. L. G. Bakker, Restriction on the strong coupling constant in the IR region from the 1D-1P splitting in bottomonium, Phys. Rev. D 70, 016007 (2004).

[399] A. Deur, Measurement of the $Q^{2}$-evolution of the Bjorken integral and extraction of an effective strong coupling constant at low $Q^{2}$, Nucl. Phys. A 755, 353 (2005) [nucl-ex/0501015].

[400] A. L. Kataev, personal communication, in S. Incerti, Ph. D dissertation. Universite Blaise Pascal, PCCF-T-9801 (1998).

[401] E. V. Shuryak and A. I. Vainshtein, Theory of Power Corrections to Deep Inelastic Scattering in Quantum Chromodynamics. 2. Q**4 Effects: Polarized Target, Nucl. Phys. B 201, 141 (1982); X. D. Ji and P. Unrau, Q**2 dependence of the proton's G1 structure function sum rule, Phys. Lett. B 333, 228 (1994) [hep-ph/9308263]; H. Kawamura, T. Uematsu, J. Kodaira and Y. Yasui, Renormalization of twist four operators in QCD Bjorken and Ellis-Jaffe sum rules, Mod. Phys. Lett. A 12, 135 (1997) [hep-ph/9603338].

[402] J. Blumlein and H. Bottcher, QCD analysis of polarized deep inelastic data and parton distributions, Nucl. Phys. B 636, 225 (2002) [hep-ph/0203155]. 
[403] X. Zheng et al. [Jefferson Lab Hall A Collaboration], Precision measurement of the neutron spin asymmetry $A_{1}^{N}$ and spin flavor decomposition in the valence quark region, Phys. Rev. Lett. 92, 012004 (2004) [nucl-ex/0308011]; X. Zheng et al. [Jefferson Lab Hall A Collaboration], Precision measurement of the neutron spin asymmetries and spin-dependent structure functions in the valence quark region, Phys. Rev. C 70, 065207 (2004) [nucl-ex/0405006]; M. Posik et al. [Jefferson Lab Hall A Collaboration], A precision measurement of the neutron twist-3 matrix element $d_{2}^{n}$ : probing color forces, Phys. Rev. Lett. 113, 022002 (2014) [arXiv:1404.4003 [nucl-ex]].

[404] E. Stein, P. Gornicki, L. Mankiewicz and A. Schafer, QCD sum rule calculation of twist four corrections to Bjorken and Ellis-Jaffe sum rules, Phys. Lett. B 353, 107 (1995) [hep-ph/9502323]; I. I. Balitsky, V. M. Braun and A. V. Kolesnichenko, Power corrections $1 / Q^{2}$ to parton sum rules for deep inelastic scattering from polarized targets, Phys. Lett. B 242, 245 (1990) [Phys. Lett. B 318, 648 (1993)] [hep-ph/9310316]; X. D. Ji and W. Melnitchouk, Spin dependent twist four matrix elements from $g_{1}$ data in the resonance region, Phys. Rev. D 56, 1 (1997) [hepph/9703363]; N. Y. Lee, K. Goeke and C. Weiss, Spin dependent twist four matrix elements from the instanton vacuum: Flavor singlet and nonsinglet, Phys. Rev. D 65, 054008 (2002) [hep-ph/0105173]; A. V. Sidorov and C. Weiss, Higher twists in polarized DIS and the size of the constituent quark, Phys. Rev. D 73, 074016 (2006) [hep-ph/0602142].

[405] G. Cvetic and C. Valenzuela, Exponentially modified QCD coupling, Phys. Rev. D 77, 074021 (2008) [arXiv:0710.4530 [hep-ph]].

[406] S. J. Brodsky, H. C. Pauli and S. S. Pinsky, "Quantum chromodynamics and other field theories on the light cone," Phys. Rept. 301, 299 (1998) [hep-ph/9705477].

[407] S. Q. Wang, X. G. Wu, Z. G. Si and S. J. Brodsky, "Predictions for the Top-Quark Forward-Backward Asymmetry at High Invariant Pair Mass Using the Principle of Maximum Conformality," Phys. Rev. D 93, no. 1, 014004 (2016) [arXiv:1508.03739 [hep-ph]].

[408] R. Horsley et al., Wilson loops to 20th order numerical stochastic perturbation theory, Phys. Rev. D 86, 054502 (2012) [arXiv:1205.1659 [hep-lat]]; C. Bauer, G. S. Bali and A. Pineda, Compelling evidence of renormalons in QCD from high order perturbative expansions, Phys. Rev. Lett. 108, 242002 (2012) [arXiv:1111.3946 [hep-ph]]; 
G. S. Bali, C. Bauer, A. Pineda and C. Torrero, Perturbative expansion of the energy of static sources at large orders in four-dimensional SU(3) gauge theory, Phys. Rev. D 87, 094517 (2013) [arXiv:1303.3279 [hep-lat]].

[409] A. Casher and L. Susskind, "Chiral magnetism (or magnetohadrochironics)," Phys. Rev. D 9, 436 (1974).

[410] S. J. Brodsky, C. D. Roberts, R. Shrock and P. C. Tandy, Confinement contains condensates, Phys. Rev. C 85 (2012) 065202. [arXiv:1202.2376 [nucl-th]].

[411] V. I. Zakharov, Renormalons as a bridge between perturbative and nonperturbative physics, Prog. Theor. Phys. Suppl. 131, 107 (1998) [hep-ph/9802416]. M. Beneke, Renormalons, Phys. Rept. 317, 1 (1999) [hep-ph/9807443]. 